Supporting Information

\title{
Catalytic Hydrogenation of a Manganese(V) Nitride to Ammonia
}

\author{
Sangmin Kim, Hongyu Zhong, Yoonsu Park, Florian Loose and Paul J. Chirik* \\ Department of Chemistry, Frick Laboratory \\ Princeton University, Princeton, NJ 08544, USA \\ pchirik@princeton.edu
}

\section{Table of Contents}

$\begin{array}{ll}\text { General Considerations } & \text { S2 }\end{array}$

$\begin{array}{ll}\text { Preparation of Rhodium Compounds } & \text { S4 }\end{array}$

$\begin{array}{ll}\text { General Catalytic Procedures and Organometallic Reactions } & \text { S7 }\end{array}$

Spectroscopic Data $\quad$ S9

$\begin{array}{lr}\text { Crystallographic Data } & \text { S27 }\end{array}$

$\begin{array}{ll}\text { Computational Analysis } & \text { S85 }\end{array}$

$\begin{array}{ll}\text { References } & \text { S90 }\end{array}$ 


\section{General Considerations}

All air- and moisture-sensitive manipulations were carried out using vacuum line, Schlenk and cannula techniques or in an MBraun inert atmosphere (nitrogen) dry box unless otherwise noted. All glassware was stored in a pre-heated oven prior to use. The solvents used for airand moisture-sensitive manipulations were dried and deoxygenated using literature procedures. ${ }^{1} \mathrm{~N}, N, N^{\prime}, N^{\prime}$-Tetramethyldiamine (TMEDA) was dried over $\mathrm{CaH}$ for 5 days, degassed and transferd under vacuum before use. The following compounds were prepared according to literature procedures: ${ }^{\mathrm{Me}} \mathrm{Phl} \quad(\mathrm{N}$-methyl-1-phenylethan-1-imine $),{ }^{2} \quad \mathbf{1},{ }^{3} \quad \mathbf{6},{ }^{3} \quad\left({ }^{\mathrm{tBu}} \mathrm{Salen}\right) \mathrm{MnN},{ }^{4}$ $\left({ }^{\mathrm{tBu}}\right.$ Salen) $\mathrm{Mn}^{15} \mathrm{~N} .^{5}$

${ }^{1} \mathrm{H}$ NMR spectra were recorded on either Bruker ADVANCE 300 or 500 spectrophotometers operating at $300.13 \mathrm{MHz}$, and $500.46 \mathrm{MHz}$, respectively. ${ }^{13} \mathrm{C}$ NMR spectra were recorded on either Bruker ADVANCE 300 or 500 spectrometer operating at $75.48 \mathrm{MHz}$ and $125.85 \mathrm{MHz}$, respectively. All ${ }^{1} \mathrm{H}$ and ${ }^{13} \mathrm{C}$ NMR chemical shifts are reported in ppm relative to $\mathrm{SiMe}_{4}$ using the ${ }^{1} \mathrm{H}$ (chloroform-d: 7.26 ppm; benzene- $d_{6}: 7.16$ ppm; THF- $d_{8}: 3.58$ and 1.72 ppm; DMSO- $d_{6}: 2.50$ ppm; MeCN- $d_{3}: 1.94 \mathrm{ppm}$ ) and ${ }^{13} \mathrm{C}$ (chloroform- $d: 77.16$ ppm; benzene- $d_{6}: 128.06 \mathrm{ppm}$; THF- $d_{8}$ : 67.21 and $25.31 \mathrm{ppm}$; $\mathrm{MeCN}-d_{3}: 118.26$ and $1.32 \mathrm{ppm}$ ) chemical shifts of the solvent as a standard. ${ }^{1} \mathrm{H}$ NMR data for diamagnetic compounds are reported as follows: chemical shift, multiplicity $(\mathrm{s}=$ singlet, $\mathrm{d}=$ doublet, $\mathrm{t}=$ triplet, $\mathrm{q}=$ quartet, $\mathrm{p}=$ pentet, $\mathrm{br}=$ broad, $\mathrm{m}=$ multiplet, app $=$ apparent, obsc $=$ obscured $),$ coupling constants $(\mathrm{Hz})$, integration, assignment. ${ }^{1} \mathrm{H}$ NMR data for paramagnetic compounds are reported as follows: chemical shift, integration, peak width at half height $(\mathrm{Hz}) \cdot{ }^{13} \mathrm{C}$ NMR data for diamagnetic compounds are reported as follows: chemical shift, number of protons attached to carbon (e.g. $\mathrm{CH}_{2}$ ), assignment.

Continuous wave EPR spectra were recorded at room temperature on an X-band Bruker EMXPlus spectrometer equipped with an EMX standard resonator and a Bruker PremiumX microwave bridge. The spectra were simulated using EasySpin for MATLAB. ${ }^{6}$ 
Elemental analyses were performed at Robinson Microlit Laboratories, Inc., in Ledgewood, NJ. Solid-state magnetic moments were determined using a Johnson Matthey Magnetic Susceptibility Balance that was calibrated with $\mathrm{HgCo}(\mathrm{SCN})_{4}$. High-resolution mass spectra were obtained at Princeton University mass spectrometry facilities using an Agilent 6210 TOF LC/MS. Infrared spectroscopy was conducted on a Thermo-Nicolet iS10 FT-IR spectrometer calibrated with a polystyrene standard.

Single crystals suitable for X-ray diffraction were coated with polyisobutylene oil in a drybox, transferred to a nylon loop and then quickly transferred to the goniometer head of a Bruker SMART APEX DUO diffractometer equipped with a molybdenum X-ray tube $(\lambda=0.71073 \AA)$ and a Cu X-ray tube $(\lambda=1.54178 \AA)$. Preliminary data revealed the crystal system. The data collection strategy was optimized for completeness and redundancy using the Bruker COSMO software suite. The space group was identified, and the data were processed using the Bruker SAINT+ program and corrected for absorption using SADABS. The structures were solved using direct methods (SHELXS) completed by subsequent Fourier synthesis and refined by fullmatrix least-squares procedures.

All DFT calculations were carried out with Gaussian09 quantum chemical package. ${ }^{7}$ Geometry optimizations were performed with B3LYP functional ${ }^{8-12}$ and the $6-31 G^{* *}$ basis set. Rhodium atom was represented using the Los Alamos LANL2DZ basis set, ${ }^{13}$ which includes relativistic effective core potentials. For those structures having various conformations, the most stable conformer was searched and utilized. Vibrational frequency calculations were carried out at the same level of theory as the geometry optimizations. The single-point calculations of the optimized geometries were performed with B3LYP functional and the basis sets including LANL2TZ(-f) for rhodium ${ }^{14-16}$ and $6-311+G^{* *}$ basis set for other atoms. 


\section{Preparation of Rhodium Compounds.}

Preparation of $\left(\eta^{5}-\mathbf{C}_{5} \mathrm{Me}_{5}\right) \mathbf{R h}\left({ }^{\mathrm{Me}} \mathrm{Phl}\right) \mathrm{Cl}$. A modified literature procedure was used. ${ }^{17} \mathrm{~A} 500 \mathrm{~mL}$ round-bottom flask was charged with $1.00 \mathrm{~g}$ of $\left[\left(\eta^{5}-\mathrm{C}_{5} \mathrm{Me}_{5}\right) \mathrm{RhCl}_{2}\right]_{2}, 640 \mathrm{mg}$ of ${ }^{\mathrm{Me}} \mathrm{Phl}$ ligand, 800 $\mathrm{mg}$ of $\mathrm{NaOAc}$, a magnetic stir bar and $150 \mathrm{~mL}$ dichloromethane. The reaction mixture was stirred at room temperature for overnight $(\sim 15 \mathrm{~h})$. The volatiles were removed in vacuo. The resulting solids were collected on the M-sized glass frit and washed with diethylether $(1 \times 20$ $\mathrm{mL})$ and hexane $(3 \times 20 \mathrm{~mL})$. The solid was collected and dried in vacuo to yield $998 \mathrm{mg}(83 \%$ yield) of an orange solid identified as $\left(\eta^{5}-\mathrm{C}_{5} \mathrm{Me}_{5}\right) \mathrm{Rh}\left({ }^{\mathrm{Me}} \mathrm{PhI}\right) \mathrm{Cl}$. Anal Calcd for $\mathrm{C}_{19} \mathrm{H}_{25} \mathrm{CINRh}$ : $\mathrm{C}$, 56.24; $\mathrm{H}, 6.21 ; \mathrm{N}, 3.45$. Found: C, 56.38; $\mathrm{H}, 6.12 ; \mathrm{N}, 3.30 .{ }^{1} \mathrm{H} \mathrm{NMR}\left(300 \mathrm{MHz}, \mathrm{CDCl}_{3}, 23{ }^{\circ} \mathrm{C}\right): \delta$ 7.79 (imine- $P h, 1 \mathrm{H}, \mathrm{d}, 7.54 \mathrm{~Hz}$ ), 7.34 (imine- $P h, 1 \mathrm{H}$, dd, $7.83 \mathrm{~Hz}$ ), 7.20 (imine- $P h, 1 \mathrm{H}, \mathrm{dd}, 8.32$, $7.54 \mathrm{~Hz}$ ), 7.00 (imine- $\mathrm{Ph}, 1 \mathrm{H}, \mathrm{dd}, 8.32,7.24 \mathrm{~Hz}), 3.78(\mathrm{NMe}, 3 \mathrm{H}, \mathrm{s}), 2.37(\mathrm{MeC}(\mathrm{NMe}) \mathrm{Ph}, 3 \mathrm{H}, \mathrm{s})$, $1.64\left(\mathrm{C}_{5} \mathrm{Me}_{5}, 15 \mathrm{H}, \mathrm{s}\right) .{ }^{13} \mathrm{C}\left\{{ }^{1} \mathrm{H}\right\} \operatorname{NMR}\left(75.48 \mathrm{MHz}, \mathrm{CDCl}_{3}, 23{ }^{\circ} \mathrm{C}\right): \delta 178.81(\mathrm{MeC}(\mathrm{NMe}) \mathrm{Ph})$, 147.44 (imine- $P h$ ), 136.20 (imine- $P h$ ), 130.47 (imine- $P h$ ), 126.82 (imine- $P h$ ), 122.20 (imine- $P h$ ), $95.5\left(\mathrm{C}_{5} \mathrm{Me}_{5}\right), 43.97(\mathrm{NMe}), 15.17(\mathrm{MeC}(\mathrm{NMe}) \mathrm{Ph}), 9.54\left(\mathrm{C}_{5} \mathrm{Me}_{5}\right) .{ }^{*}$ One carbon was not located.

Preparation of 2. A $250 \mathrm{~mL}$ round-bottom flask was charged with $573 \mathrm{mg}$ of $\left(\eta^{5}\right.$ $\left.\mathrm{C}_{5} \mathrm{Me}_{5}\right) \mathrm{Rh}\left({ }^{\mathrm{Me}} \mathrm{PhI}\right) \mathrm{Cl}$, a magnetic stir bar and $100 \mathrm{~mL}$ THF in an $\mathrm{N}_{2}$-filled glovebox. To the mixture, $1.48 \mathrm{~mL}$ of $\mathrm{LiHBEt}_{3}$ solution (1M THF, 1.05 equiv) was added dropwise. The reaction mixture was stirred at room temperature for 2 hours. The volatiles were removed in vacuo. The resulting solids were dissolved in toluene and filtered through Celite. The volatiles of the toluene filtrate were removed in vacuo and the resulting solids were collected on the M-sized glass frit and washed with pentane $(20 \mathrm{~mL})$ and diethylether $(3 \times 2 \mathrm{~mL})$. The collected solid on the frit was dried in vacuo to yield $200 \mathrm{mg}$ (38 \% yield) of a yellow solid identified as 2. Anal Calcd for $\mathrm{C}_{19} \mathrm{H}_{26} \mathrm{NRh}$ : C, 61.46; $\mathrm{H}, 7.06 ; \mathrm{N}, 3.77$. Found: $\mathrm{C}, 61.38 ; \mathrm{H}, 6.81 ; \mathrm{N}, 3.66$. IR ( $\mathrm{v}_{\mathrm{RhH}}, \mathrm{KBr}$ pellet): $1967 \mathrm{~cm}^{-1} .{ }^{1} \mathrm{H}$ NMR $\left(300 \mathrm{MHz}, \mathrm{THF}-d_{8}, 23^{\circ} \mathrm{C}\right.$ ): $\delta 7.65$ (imine- $P h, 1 \mathrm{H}, \mathrm{d}, 7.47 \mathrm{~Hz}$ ), 7.30 (imine$P h, 1 \mathrm{H}, \mathrm{d}, 7.78 \mathrm{~Hz}$ ), 6.94 (imine- $P h, 1 \mathrm{H}, \mathrm{dd}, 8.05,7.46 \mathrm{~Hz}$ ), 6.80 (imine- $P h, 1 \mathrm{H}, \mathrm{dd}, 8.37,7.41$ 
$\mathrm{Hz}), 3.71(\mathrm{NMe}, 3 \mathrm{H}, \mathrm{s}), 2.26(\mathrm{MeC}(\mathrm{NMe}) \mathrm{Ph}, 3 \mathrm{H}, \mathrm{s}), 1.86\left(\mathrm{C}_{5} \mathrm{Me}_{5}, 15 \mathrm{H}, \mathrm{s}\right),-13.45(\mathrm{RhH}, \mathrm{d}, 33.09$ $\mathrm{Hz}) .{ }^{13} \mathrm{C}\left\{{ }^{1} \mathrm{H}\right\} \operatorname{NMR}\left(75.48 \mathrm{MHz}, \mathrm{THF}-d_{8}, 23^{\circ} \mathrm{C}\right): \delta 173.93$ (MeC(NMe)Ph), 136.92 (imine-Ph), 127.71 (imine- $P h), 126.58$ (imine- $P h), 119.84$ (imine- $P h), 95.48\left(C_{5} \mathrm{Me}_{5}\right), 45.99(\mathrm{NMe}), 13.64$ (MeC(NMe)Ph), $10.35\left(\mathrm{C}_{5} \mathrm{Me}_{5}\right) .{ }^{*}$ Two carbons were not located.

Preparation of $\mathbf{2 - d _ { 1 }}$. The same procedure for $\mathbf{2}$ was utilized except that $\mathrm{LiDBEt}_{3}$ solution was used in place of $\mathrm{LiHBEt}_{3} .60 \%$ D-incorporated $\left(\eta^{5}-\mathrm{C}_{5} \mathrm{Me}_{5}\right) \mathrm{Rh}\left({ }^{\mathrm{Me}} \mathrm{Phl}\right) \mathrm{D}$ was obtained.

Preparation of 3. A $180 \mathrm{~mL}$ thick-walled glassware was charged with $300 \mathrm{mg}$ of $\mathbf{1}$, a magnetic stir bar and $20 \mathrm{~mL}$ THF in an $\mathrm{N}_{2}$-filled glovebox. The headspace of the glassware was replaced with 4 atm $\mathrm{H}_{2}$ while the mixture was frozen using liquid nitrogen. The reaction mixture was thawed and then stirred at $70{ }^{\circ} \mathrm{C}$ for 5 days. The reaction vessel was brought into the glovebox and the volatiles were removed in vacuo. The resulting solids were triturated with pentane $(3 \mathrm{x}$ $10 \mathrm{~mL})$ and collected on the M-sized glass frit and washed with pentane $(3 \times 10 \mathrm{~mL})$. The collected solid on the frit was dried in vacuo to yield $20 \mathrm{mg}\left(6 \%\right.$ yield) of 3 as a yellow solid. ${ }^{1} \mathrm{H}$ NMR (300 MHz, benzene- $d_{6}, 23^{\circ} \mathrm{C}$ ): $\delta 8.13$ (imine- $P h, 1 \mathrm{H}, \mathrm{d}, 7.53 \mathrm{~Hz}$ ), 7.34 (imine- $P h, 1 \mathrm{H}$, dd, 7.91, 7.04 Hz), 7.25 (imine- $P h, 1 \mathrm{H}, \mathrm{d}, 7.22 \mathrm{~Hz}$ ), 7.10 (imine- $P h, 1 \mathrm{H}$, dd, 8.09, 7.04 Hz), 3.83 (imine-Ph, 2H, m), 2.19 (imine-Ph, 2H, m), $1.87\left(\mathrm{C}_{5} \mathrm{Me}_{5}, 15 \mathrm{H}, \mathrm{s}\right), 1.31$ (imine-Ph, 2H, m), 1.21 (imine-Ph, 2H, m), -12.71 (Rh- H, d, $33.51 \mathrm{~Hz}) .{ }^{1} \mathrm{H}$ NMR $\left(300 \mathrm{MHz}, \mathrm{THF}-\mathrm{d}_{8}, 23^{\circ} \mathrm{C}\right): \delta 7.63$ (imine- $P h, 1 \mathrm{H}, \mathrm{d}, 7.49 \mathrm{~Hz}$ ), 7.25 (imine- $P h, 1 \mathrm{H}, \mathrm{d}, 7.67 \mathrm{~Hz}$ ), 6.93 (imine- $P h, 1 \mathrm{H}$, dd, 8.48, 6.94 $\mathrm{Hz}$ ), 6.78 (imine- $P h, 1 \mathrm{H}, \mathrm{dd}, 8.03,7.21 \mathrm{~Hz}$ ), 3.97 (imine- $\mathrm{Ph}, 2 \mathrm{H}, \mathrm{m}$ ), 2.76 (imine-Ph, 2H, m), $1.85\left(\mathrm{C}_{5} \mathrm{Me}_{5}, 15 \mathrm{H}, \mathrm{s}\right), 1.79$ (imine-Ph, 4H, m), -13.43 (Rh- $\left.\mathrm{H}, \mathrm{d}, 33.15 \mathrm{~Hz}\right) .{ }^{13} \mathrm{C}\left\{{ }^{1} \mathrm{H}\right\} \mathrm{NMR}(125.85$ $\mathrm{MHz}$, benzene- $\left.d_{6}, 23^{\circ} \mathrm{C}\right): \delta 184.4(\mathrm{Rh}-\mathrm{C}(\mathrm{Ph})), 173.35(\mathrm{~N}=\mathrm{C}($ imine $)), 146.34$ (imine- $\left.\mathrm{Ph}\right), 137.20$ (imine-Ph), 125.55 (imine-Ph), 120.04 (imine-Ph), $95.06\left(C_{5} \mathrm{Me}_{5}\right), 57.36$ (imine-Ph), 26.53 (imine-Ph), 24.20 (imine-Ph), 19.21 (imine- $\mathrm{Ph}), 10.62\left(\mathrm{C}_{5} \mathrm{Me}_{5}\right) .{ }^{*}$ One carbon was not located.

Preparation of 5. A $20 \mathrm{~mL}$ vial was charged with $49 \mathrm{mg}$ of 2 (1.0 equiv, $0.132 \mathrm{mmol}), 47 \mathrm{mg}$ of TEMPO (2.3 equiv, $0.300 \mathrm{mmol}$ ), a magnetic stir bar and $3 \mathrm{~mL}$ benzene in an $\mathrm{N}_{2}$-filled glovebox. The reaction mixture was stirred at $23^{\circ} \mathrm{C}$ for 2 hours and kept in the vial for $46 \mathrm{~h}$ without stirring. 
The solvent was removed by decanting and the remaining solids were washed with cold chloroform $(2 \times 1 \mathrm{~mL})$, dried and collected to yield $20 \mathrm{mg}(41 \%$ yield, $0.027 \mathrm{mmol})$ of red solid identified as 5. Recrystallization from diethyl ether generated red crystals suitable for X-ray diffraction. ${ }^{1} \mathrm{H}$ NMR $\left(400 \mathrm{MHz}, \mathrm{CDCl}_{3}, 23^{\circ} \mathrm{C}\right.$ ): $\delta 7.65$ (imine- $\left.\mathrm{Ph}, 2 \mathrm{H}, \mathrm{m}\right), 7.31$ (imine- $P h, 2 \mathrm{H}, \mathrm{m}$ ), 6.95 (imine- $\mathrm{Ph}, 4 \mathrm{H}, \mathrm{m}), 3.24\left(\mathrm{Rh}-\mathrm{C}_{2} \mathrm{H}_{2}\right.$-imine, $\left.2 \mathrm{H}, \mathrm{m}\right), 2.18\left(\mathrm{Rh}-\mathrm{C}_{2} \mathrm{H}_{2}\right.$-imine, $\left.2 \mathrm{H}, \mathrm{s}\right), 1.68\left(\mathrm{C}_{5} \mathrm{Me}_{5}\right.$ + NMe,36H, m). ${ }^{13} \mathrm{C}\left\{{ }^{1} \mathrm{H}\right\} \operatorname{NMR}\left(125.85 \mathrm{MHz}, \mathrm{CDCl}_{3}, 23{ }^{\circ} \mathrm{C}\right): \delta 184.0$ (N=C(imine)), 180.5 (Rh$C(P h), d, 34.66 \mathrm{~Hz}$ ), 150.7 (imine-Ph), 136.7 (imine-Ph), 125.6 (imine-Ph), 124.2 (imine-Ph), 120.0 (imine- $P h), 94.7\left(C_{5} \mathrm{Me}_{5}\right), 40.1$ (NMe), $17.2\left(\mathrm{Rh}-\mathrm{C}_{2} \mathrm{H}_{2}\right.$-imine, d, $\left.25.56 \mathrm{~Hz}\right), 9.5\left(\mathrm{C}_{5} \mathrm{Me}_{5}\right)$.

Preparation of $\left[\left(\eta^{5}-C_{5} M_{5}\right) R h\left({ }^{M e} \mathrm{Phl}\right)(\mathrm{MeCN})\right]\left[\mathrm{BArF}_{24}\right]$. A $20 \mathrm{~mL}$ vial was charged with $50 \mathrm{mg}$ of $\left(\eta^{5}-\mathrm{C}_{5} \mathrm{Me}_{5}\right) \mathrm{Rh}\left({ }^{\mathrm{Me}} \mathrm{Phl}\right) \mathrm{Cl}(1.0$ equiv, $0.123 \mathrm{mmol}), 115 \mathrm{mg}$ of $\mathrm{NaBArF}_{24}$ (1.05 equiv, $\left.0.129 \mathrm{mmol}\right)$ and $10 \mathrm{~mL}$ DCM under air. The reaction mixture was stirred at $23{ }^{\circ} \mathrm{C}$ for $10 \mathrm{~min}$ and $1 \mathrm{~mL}$ of $\mathrm{MeCN}$ was added to the reaction mixture in one portion. The reaction mixture was stirred for another $10 \mathrm{~min}$. The volatiles were removed in vacuo and $1 \mathrm{~mL}$ of $\mathrm{Et}_{2} \mathrm{O}$ was added to the resulting reaction mixture. $\mathrm{Et}_{2} \mathrm{O}$ was slowly evaporated at $23^{\circ} \mathrm{C}$ until the volume reached to approximately $0.2 \mathrm{~mL}$ and the liquid was removed by decanting. The remaining solid was dried under vacuum and collected to yield $30 \mathrm{mg}(23 \%$ yield, $0.0235 \mathrm{mmol})$ of $\left[\left(\eta^{5}-\right.\right.$ $\left.\left.\mathrm{C}_{5} \mathrm{Me}_{5}\right) \mathrm{Rh}\left({ }^{\mathrm{Me}} \mathrm{Phl}\right)(\mathrm{MeCN})\right]\left[\mathrm{BArF}_{24}\right]$ as an orange crystal suitable for X-ray diffraction. IR $\left(\mathrm{V}_{\mathrm{MeCN}}\right.$, $\mathrm{KBr}$ pellet): 2294, $2318 \mathrm{~cm}^{-1}$. Two MeCN signals were also observed for 1 in the literature. ${ }^{3}{ }^{1} \mathrm{H}$ NMR (400 MHz, MeCN- $\left.d_{3}, 23^{\circ} \mathrm{C}\right): \delta 7.76$ (imine-Ph, $\left.1 \mathrm{H}, \mathrm{d}, 7.6 \mathrm{~Hz}\right), 7.67\left(\mathrm{BArF}_{24}, 8 \mathrm{H}, \mathrm{s}\right), 7.64$ $\left(\mathrm{BArF}_{24}, 4 \mathrm{H}, \mathrm{s}\right.$ ), 7.48 (imine- $P h, 1 \mathrm{H}, \mathrm{d}, 7.7 \mathrm{~Hz}$ ), 7.25 (imine- $P h, 1 \mathrm{H}, \mathrm{t}, 6.8 \mathrm{~Hz}$ ), 7.11 (imine- $P h$, 1H, t, $7.9 \mathrm{~Hz}), 3.71(\mathrm{NMe}, 3 \mathrm{H}, \mathrm{s}), 2.37(\mathrm{MeC}(\mathrm{NMe}) \mathrm{Ph}, 3 \mathrm{H}, \mathrm{s}), 2.12(\mathrm{MeCN}, 3 \mathrm{H}, \mathrm{s}), 1.62\left(\mathrm{C}_{5} \mathrm{Me}_{5}\right.$, $15 \mathrm{H}, \mathrm{s}) .{ }^{13} \mathrm{C}\left\{{ }^{1} \mathrm{H}\right\}$ NMR $\left(125.85 \mathrm{MHz}, \mathrm{MeCN}-d_{3}, 23{ }^{\circ} \mathrm{C}\right): \delta 183.4(\mathrm{Rh}-\mathrm{C}(\mathrm{Ph})), 179.4(\mathrm{~N}=\mathrm{C}($ imine $)$, d, $30.45 \mathrm{~Hz}), 162.5\left(\mathrm{BArF}_{24}, \mathrm{q}, 49.70 \mathrm{~Hz}\right), 149.1$ (imine- $\left.P h\right), 136.8$ (imine- $\left.P h\right), 135.6\left(\mathrm{BArF}_{24}\right.$, br s), 131.9 (imine-Ph), $129.9\left(\mathrm{BArF}_{24}, \mathrm{qq}, 31.72,2.83 \mathrm{~Hz}\right), 128.6$ (imine-Ph), $125.4\left(\mathrm{BArF}_{24}, \mathrm{q}\right.$, $271.81 \mathrm{~Hz}$ ), 124.6 (imine-Ph), $118.6\left(\mathrm{BArF}_{24}\right.$, br sept, $\left.3.91 \mathrm{~Hz}\right), 99.0\left(\mathrm{C}_{5} \mathrm{Me}_{5}, \mathrm{~d}, 6.14 \mathrm{~Hz}\right), 44.9$ (NMe), $15.7(\mathrm{MeC}(\mathrm{NMe}) \mathrm{Ph}), 9.4\left(\mathrm{C}_{5} \mathrm{Me}_{5}\right)$. ${ }^{*}$ Two carbons of the coordinating MeCN are missing. 


\section{General Catalytic Procedures and Organometallic Reactions}

General Procedure for Hydrogenation of ( ${ }^{\mathrm{tBu}}$ Salen)MnN. In a typical experiment, a 20-mL thick-walled glassware was charged with $12 \mathrm{mg}$ of ( ${ }^{\mathrm{tBu}}$ Salen) $\mathrm{MnN}(0.02 \mathrm{mmol}, 1.0$ equiv), 10 mol\% of the rhodium precatalyst $(0.002 \mathrm{mmol})$, additive $(0.04 \mathrm{mmol}, 2.0$ equiv), a magnetic stir bar and $0.5 \mathrm{~mL}$ THF $(0.04 \mathrm{M})$ in an $\mathrm{N}_{2}$-filled glovebox. The headspace was replaced with 4 atm $\mathrm{H}_{2}$ while the contents of the vessel were frozen in liquid nitrogen. The reaction vessel was wrapped with aluminum foil and the reaction mixture was stirred at room temperature for 5 days. The reaction mixture was then frozen by submersion of the vessel in liquid nitrogen and $\mathrm{H}_{2}$ gas was removed under vacuum. By vacuum transfer, all the remaining volatiles were transferred to another $20-\mathrm{mL}$ thick-walled glassware charged with $1 \mathrm{~mL}$ of $4.0 \mathrm{M} \mathrm{HCl}$ solution in dioxane. The mixture was thawed and stirred for $10 \mathrm{~min}$ and all the volatiles were removed in vacuo. The resulting product was dissolved in DMSO- $d_{6}$ and then analyzed by ${ }^{1} \mathrm{H}$ NMR spectroscopy using $5.0 \mu \mathrm{L}$ of 1,2 -dichloroethane as an internal standard.

Chlorination of Non-Volatile Residue after Hydrogenation of ( ${ }^{\text {tBu }}$ Salen)MnN to Form ( ${ }^{\mathrm{tBu}}$ Salen) $\mathrm{MnCl}$. To the nonvolatile residue from the reaction was added $20 \mathrm{mg}$ of $\mathrm{C}_{2} \mathrm{Cl}_{6}(80$ $\mu \mathrm{mol}, 4 \mathrm{eq}$ ) dissolved in $1 \mathrm{~mL}$ THF. The resulting mixture was stirred at $50{ }^{\circ} \mathrm{C}$ for $48 \mathrm{hrs}$. The reaction was then cooled and the volatiles were removed under vacuum. The resulting mixture was diluted to $25.0 \mathrm{~mL}$ with $\mathrm{CH}_{2} \mathrm{Cl}_{2}$ in a volumetric flask. The resulting standard solution was transferred to an $1 \mathrm{~cm} \AA \sim 1 \mathrm{~cm}$ cuvette and subject to UV-Vis study. The product solution was then recombined and concentrated to dryness in vacuo for further transformation (vide infra).

Conversion of In-Situ Generated ( $\left.{ }^{\mathrm{B} u} \mathrm{Salen}\right) \mathrm{MnCl}$ into ( $\left.{ }^{\mathrm{tBu}} \mathrm{Salen}\right) \mathrm{MnN}$. The crude product of oxidative chlorination (vide supra) was dissolved in $2 \mathrm{~mL} \mathrm{CH}_{2} \mathrm{Cl}_{2} / \mathrm{MeOH}$ (4:1). To the solution was added 5 drops of aq. $\mathrm{NH}_{3}$, after which the reaction was stirred at room temperature for 15 minutes. 20 drops of Clorox bleach was then added and the reaction was stirred at room temperature for $16 \mathrm{hrs}$. The organic phase of the reaction was then filtered through a pipette containing 1 inch of neutral alumina. After rinsing the plug with additional $\mathrm{CH}_{2} \mathrm{Cl}_{2}$, the resulting 
solution was dried in vacuo and subject to NMR analysis with 1,2-dichoroethane as an internal standard. The yield of ( ${ }^{\mathrm{tBu}}$ Salen)MnN obtained is $40 \%$.

Stoichiometric Reaction of ( ${ }^{\mathrm{tBu}}$ Salen)MnN with 1 or 2 . A $20-\mathrm{mL}$ vial was charged with $25 \mathrm{mg}$ of $\mathrm{MnN}\left({ }^{\mathrm{tBu}}\right.$ Salen) and $8 \mathrm{mg}$ of a rhodium complex (1 or 2$)$ in an $\mathrm{N}_{2}$-filled glovebox. Then, $2 \mathrm{~mL}$ THF was added and the mixture was stirred at room temperature for $30 \mathrm{~min}$. Then, all the volatiles were removed under vacuum. The resulting compound was then dissolved in THF- $d_{8}$ and then analyzed by ${ }^{1} \mathrm{H}$ NMR spectroscopy or was dissolved in $1 \mathrm{~mL}$ toluene and analyzed by EPR spectroscopy. The THF- $d_{8}$ solution was transferred to a J-Young tube and the headspace was charged with 4 atm $\mathrm{H}_{2}$. Then, the reaction was monitored by ${ }^{1} \mathrm{H}$ NMR spectroscopy. For IR spectroscopy, the solution was dropped on $\mathrm{KBr}$ and dried. The resulting $\mathrm{KBr}$ was grinded and used for making the $\mathrm{KBr}$ pellet.

The stoichiometric reactions in $\mathrm{MeCN}$ and benzene were conducted similar except for use of different solvents as depicted.

Reaction of 2 and TEMPO. A 20-mL vial was charged with $254 \mathrm{mg}$ of 2, $11 \mathrm{mg}$ of TEMPO, a magnetic stir bar and $1 \mathrm{~mL}$ THF- $d_{8}$ in an $\mathrm{N}_{2}$-filled glovebox and then stirred for $30 \mathrm{~min} .0 .5 \mathrm{~mL}$ of the solution was transferred to a J-Young tube and then analyzed by ${ }^{1} \mathrm{H}$ NMR spectroscopy. To the headspace, 4 atm $\mathrm{H}_{2}$ was added and the reaction was monitored by ${ }^{1} \mathrm{H}$ NMR spectroscopy. The other $0.5 \mathrm{~mL}$ of the solution was for EPR analysis. The volatiles were removed under vacuum and $0.5 \mathrm{~mL}$ toluene was added to the resulting mixture. This mixture was then analyzed by EPR spectroscopy.

Reaction of 2 and TBD. A J-Young tube was charged with $8 \mathrm{mg}$ of 2 (1.0 equiv, $0.0215 \mathrm{mmol}$ ), $18 \mathrm{mg}$ of TBD (6.0 equiv, $0.129 \mathrm{mmol})$ and $0.45 \mathrm{~mL}$ of THF- $d_{8}$. The mixture was placed on the bench without stirring at $23^{\circ} \mathrm{C}$ for 12 days. The resulting mixture was monitored by ${ }^{1} \mathrm{H}$ NMR spectroscopy to check the completeness of the reaction and the analysis of the reaction mixture followed the literature. ${ }^{18}$ 


\section{Spectroscopic Data}

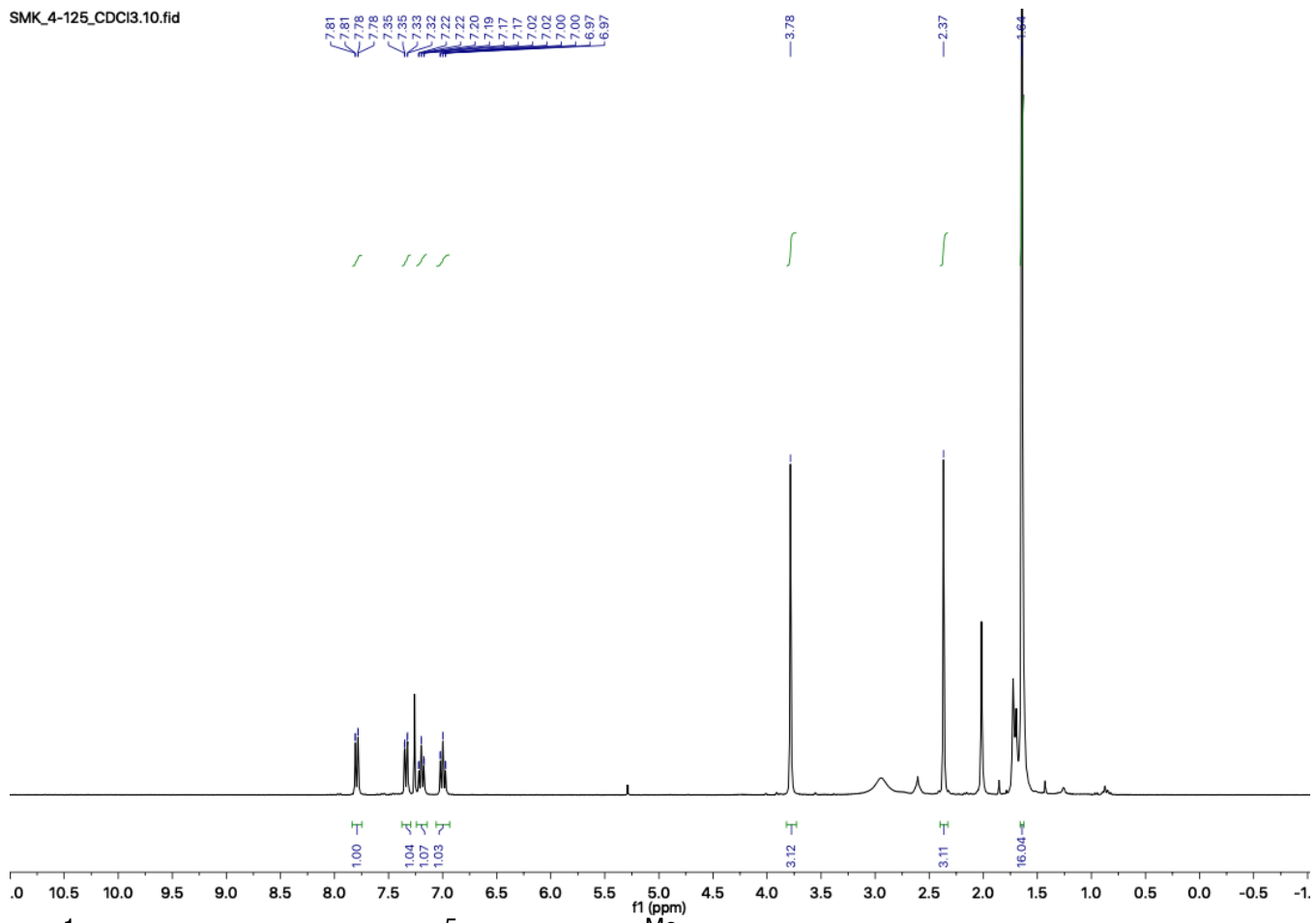

Figure S1. ${ }^{1} \mathrm{H}$ NMR spectrum of $\left(\eta^{5}-\mathrm{C}_{5} \mathrm{Me}_{5}\right) \mathrm{Rh}\left({ }^{\mathrm{M}}{ }^{\mathrm{Ph}} \mathrm{Ph}\right) \mathrm{Cl}$ in $\mathrm{CDCl}_{3}$.

SMK_4-125_C_CDC13.10.fid

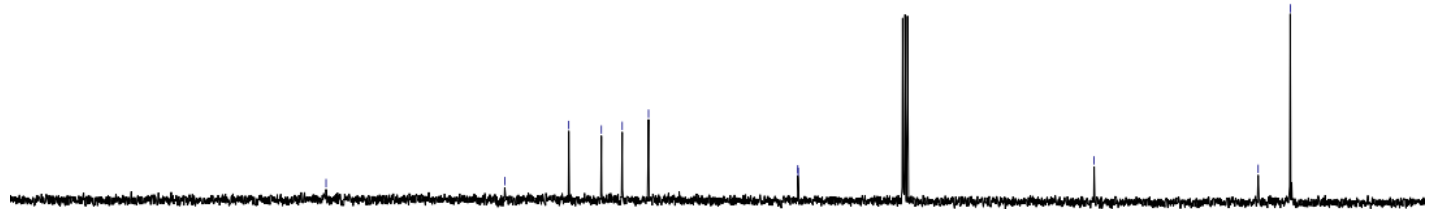

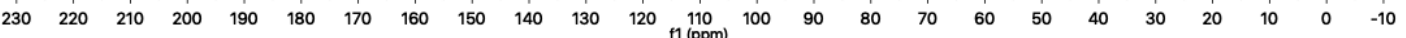

Figure S2. ${ }^{13} \mathrm{C}$ NMR spectrum of $\left(\eta^{5}-\mathrm{C}_{5} \mathrm{Me}_{5}\right) \mathrm{Rh}\left({ }^{(\mathrm{Me}} \mathrm{Phl}\right) \mathrm{Cl}$ in $\mathrm{CDCl}_{3}$. 


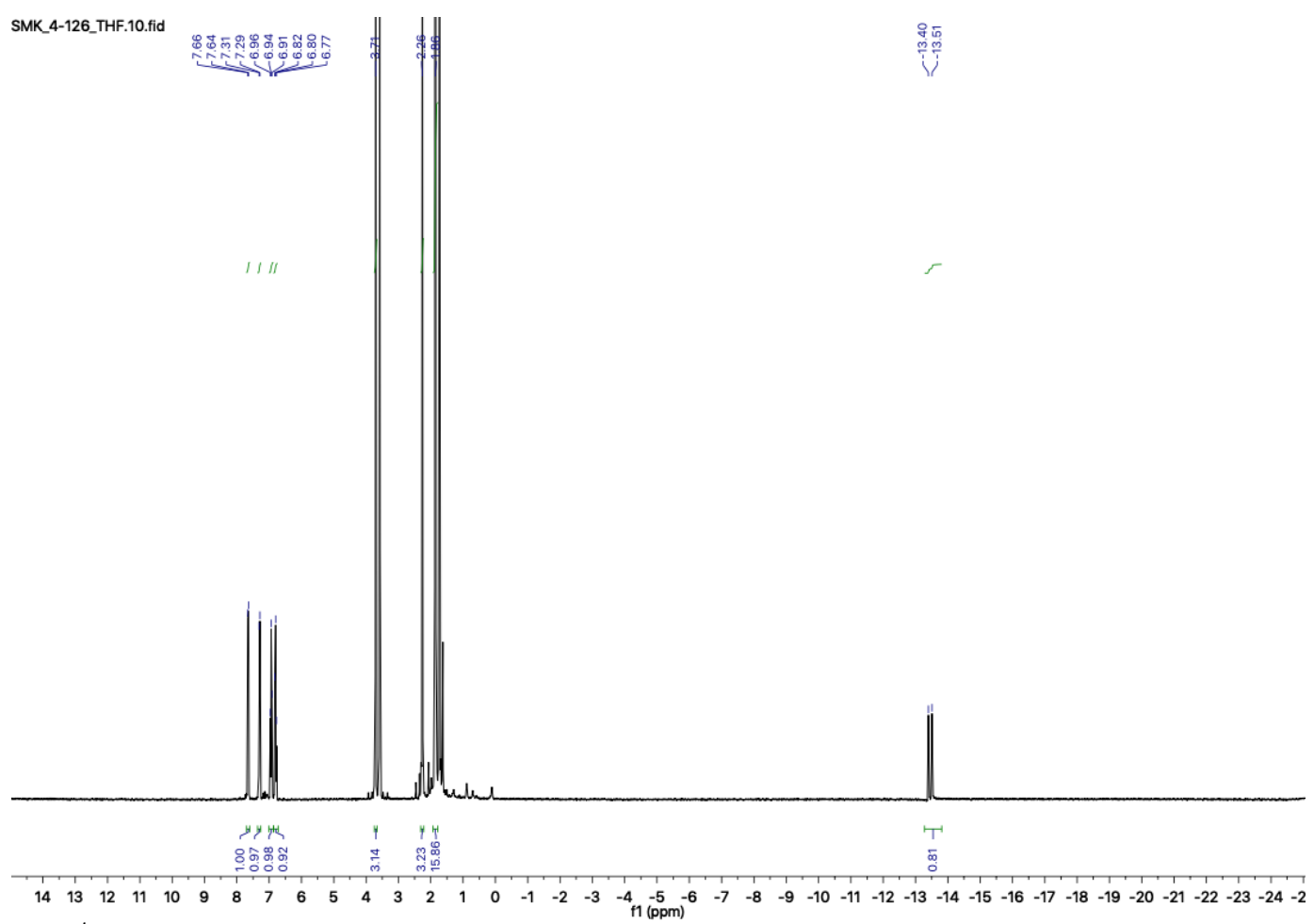

Figure S3. ${ }^{1} \mathrm{H}$ NMR spectrum of 2 in THF- $d_{8}$.

SMK_4-126_C_THF.10.fid

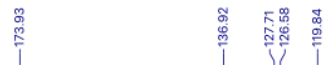

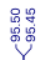
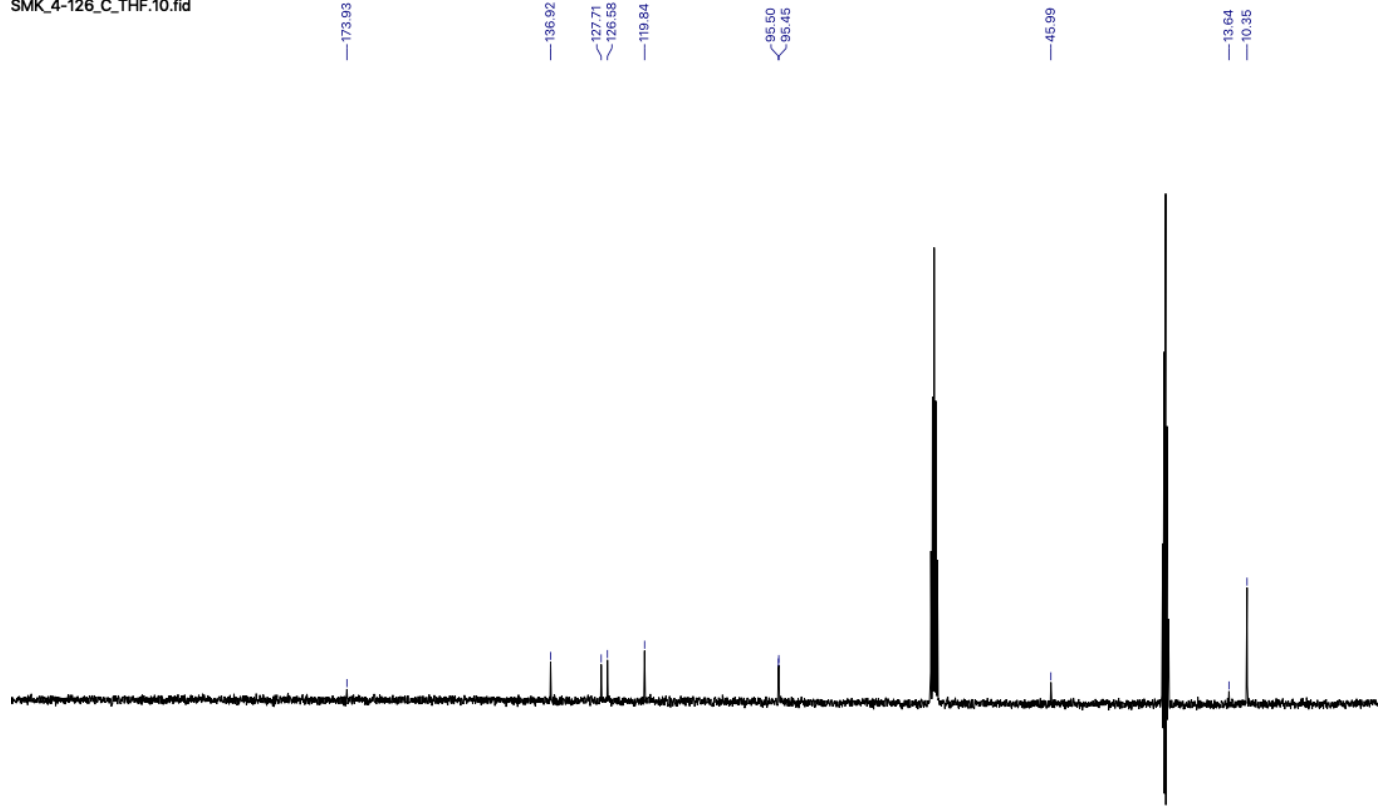

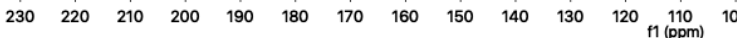
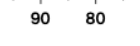

7060

50

Figure S4. ${ }^{13} \mathrm{C}$ NMR spectrum of 2 in THF- $d_{8}$. 


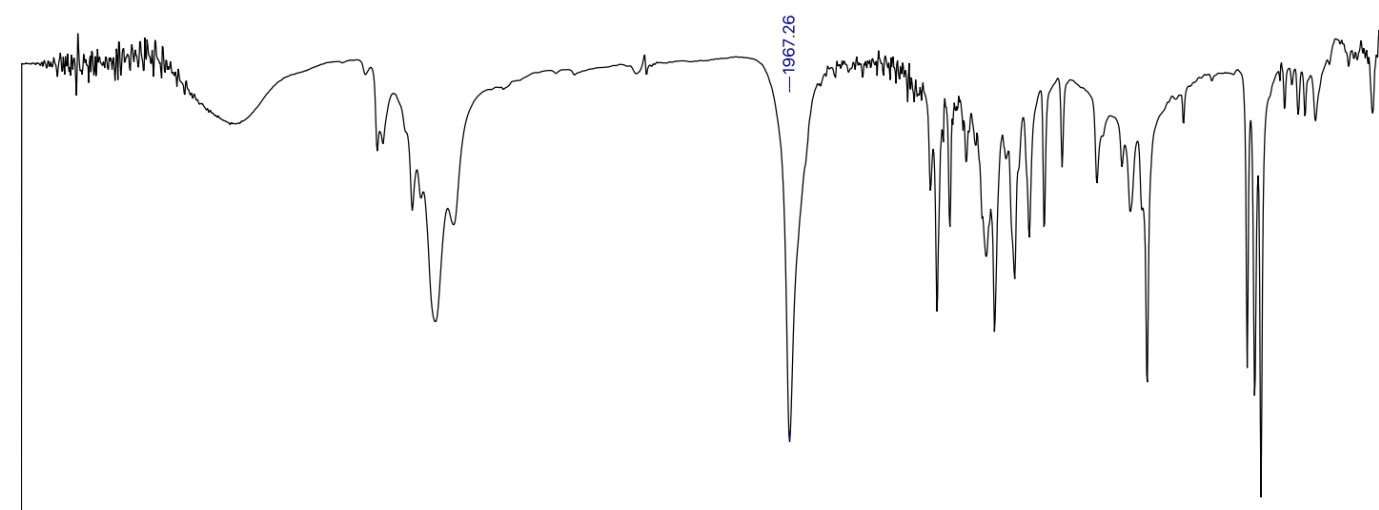

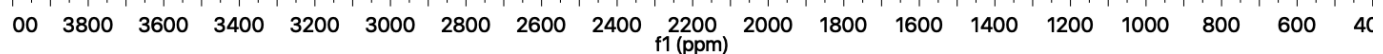
Figure S5. IR spectrum of 2 ( $\mathrm{KBr}$ pellet).

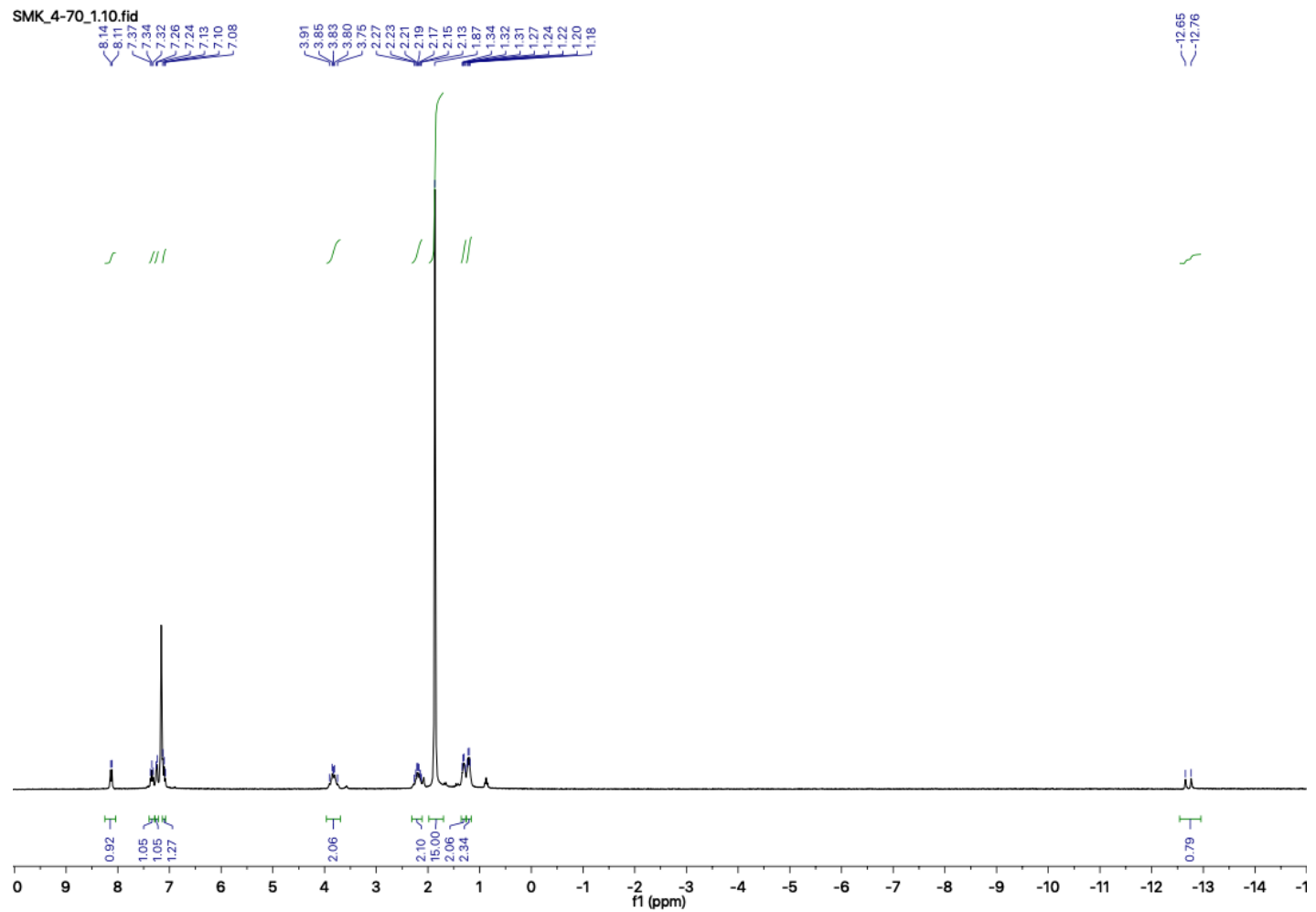

Figure S6. ${ }^{1} H$ NMR spectrum of 3 in benzene- $d_{6}$. 


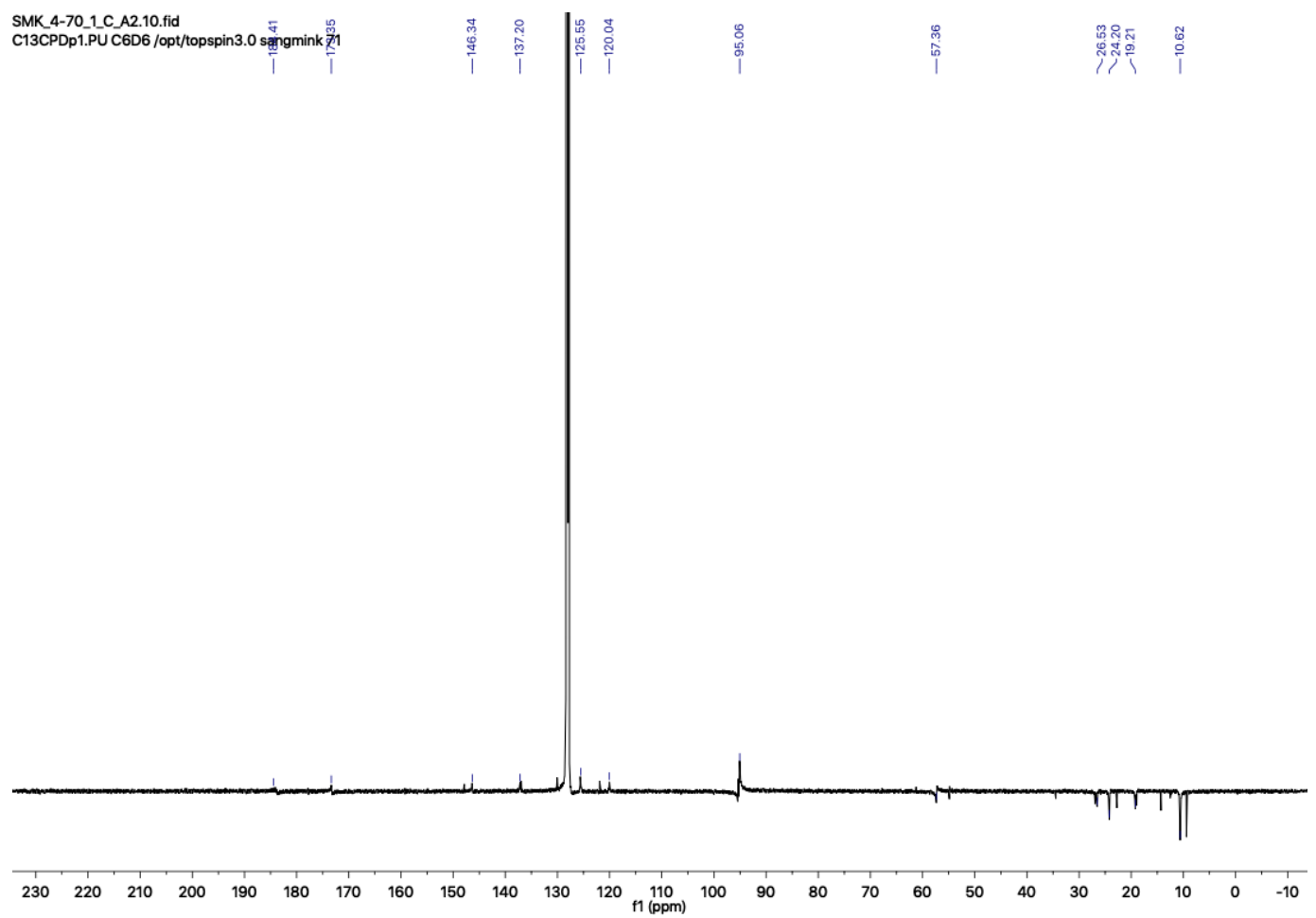

Figure S7. ${ }^{13} \mathrm{C}$ NMR spectrum of $\mathbf{3}$ in benzene- $d_{6}$.

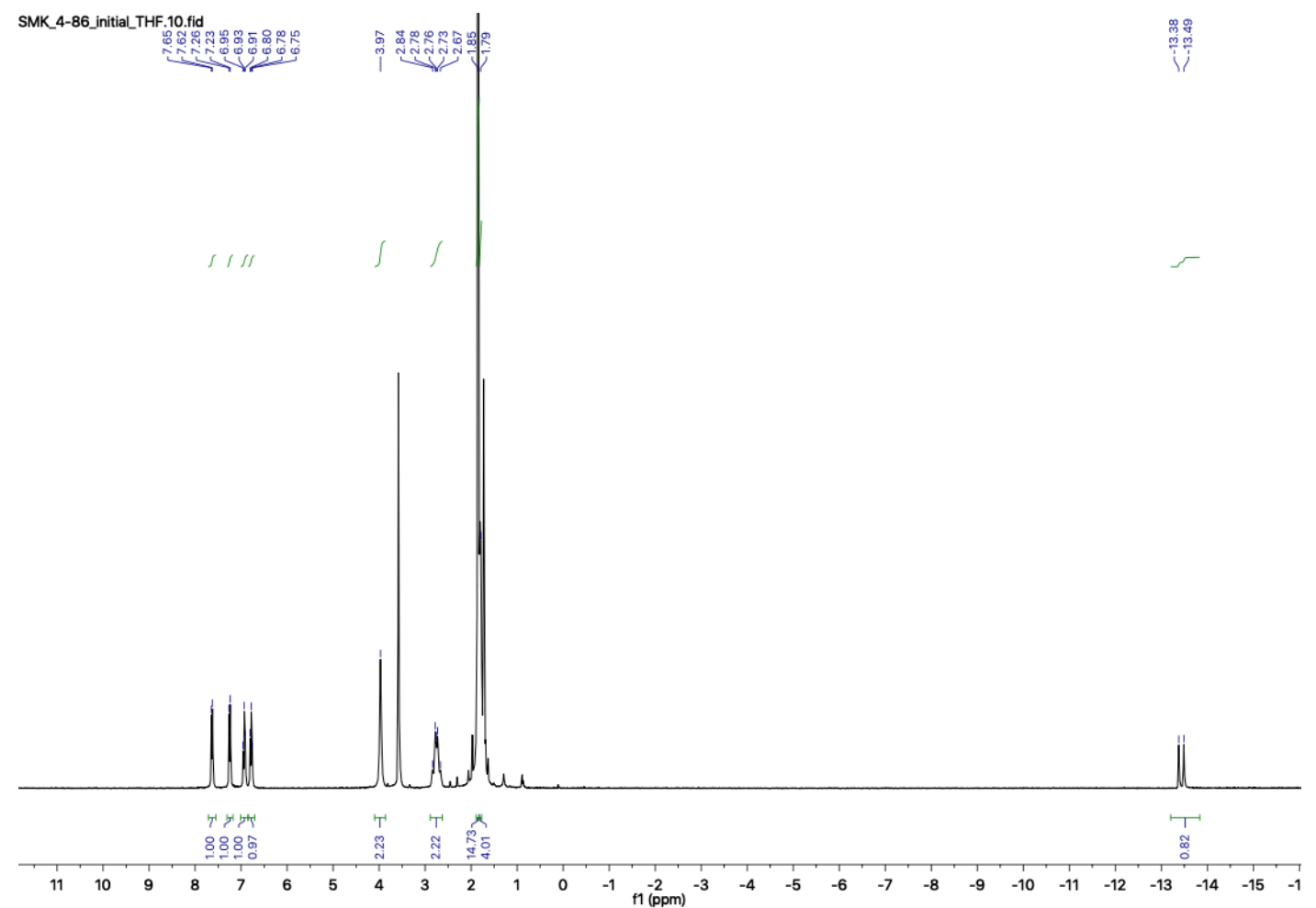

Figure S8. ${ }^{1} \mathrm{H}$ NMR spectrum of 3 in THF- $d_{8}$. 


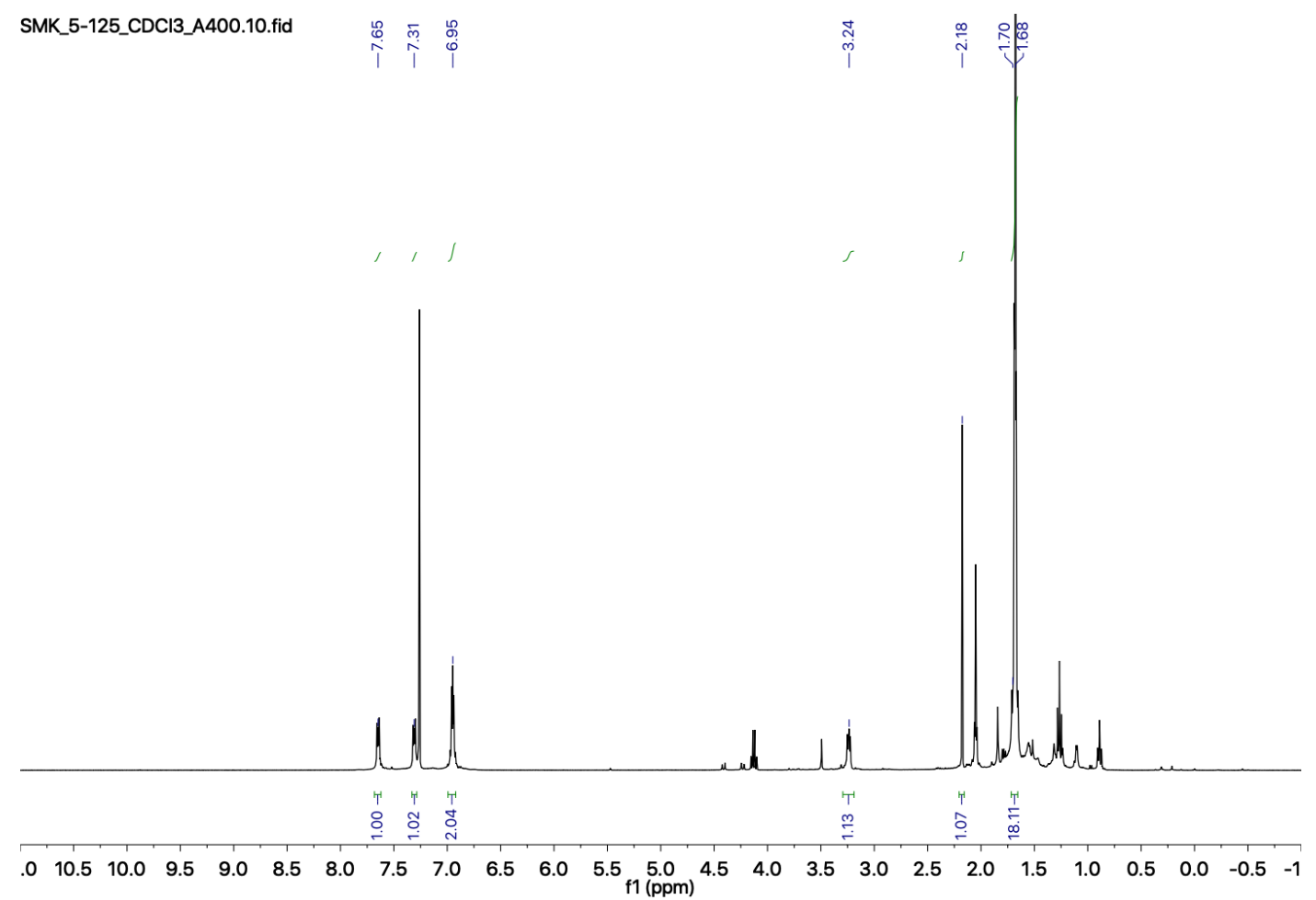

Figure S9. ${ }^{1} \mathrm{H}$ NMR spectrum of 5 in $\mathrm{CDCl}_{3}$.

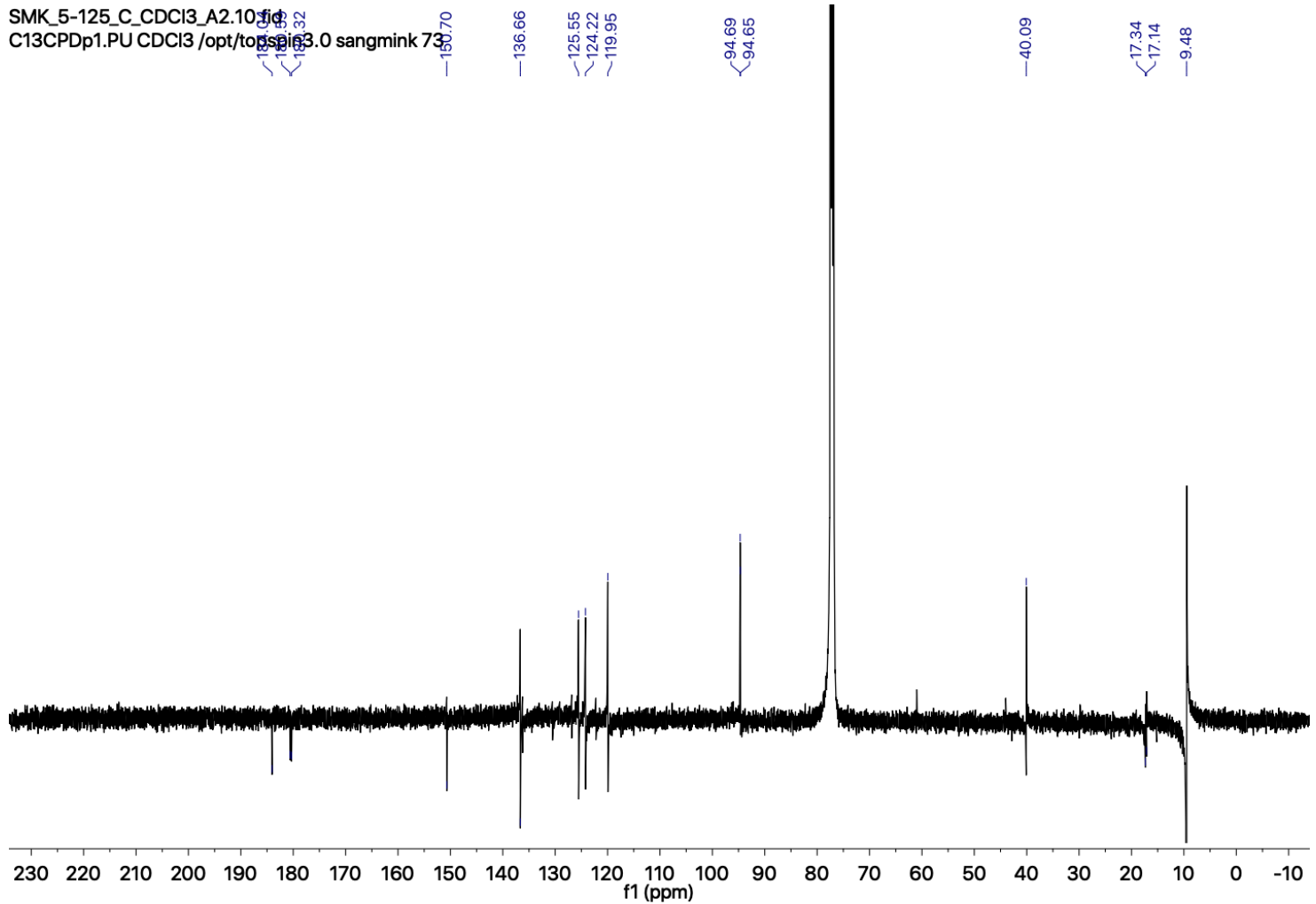

Figure $\mathrm{S} 10 .{ }^{13} \mathrm{C}$ NMR spectrum of 5 in $\mathrm{CDCl}_{3}$. 


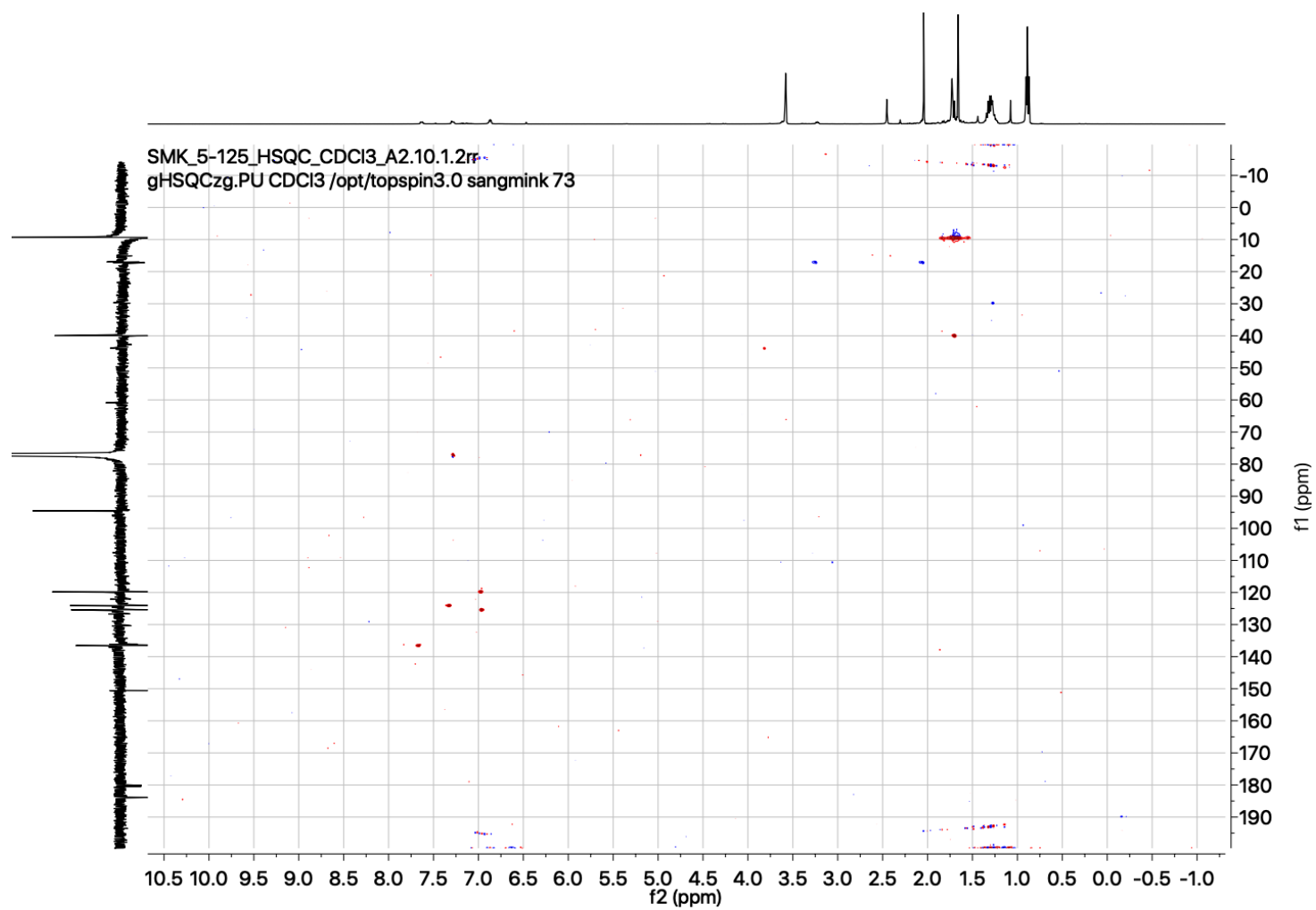

Figure S11. HSQC NMR spectrum of 5 in $\mathrm{CDCl}_{3}$.

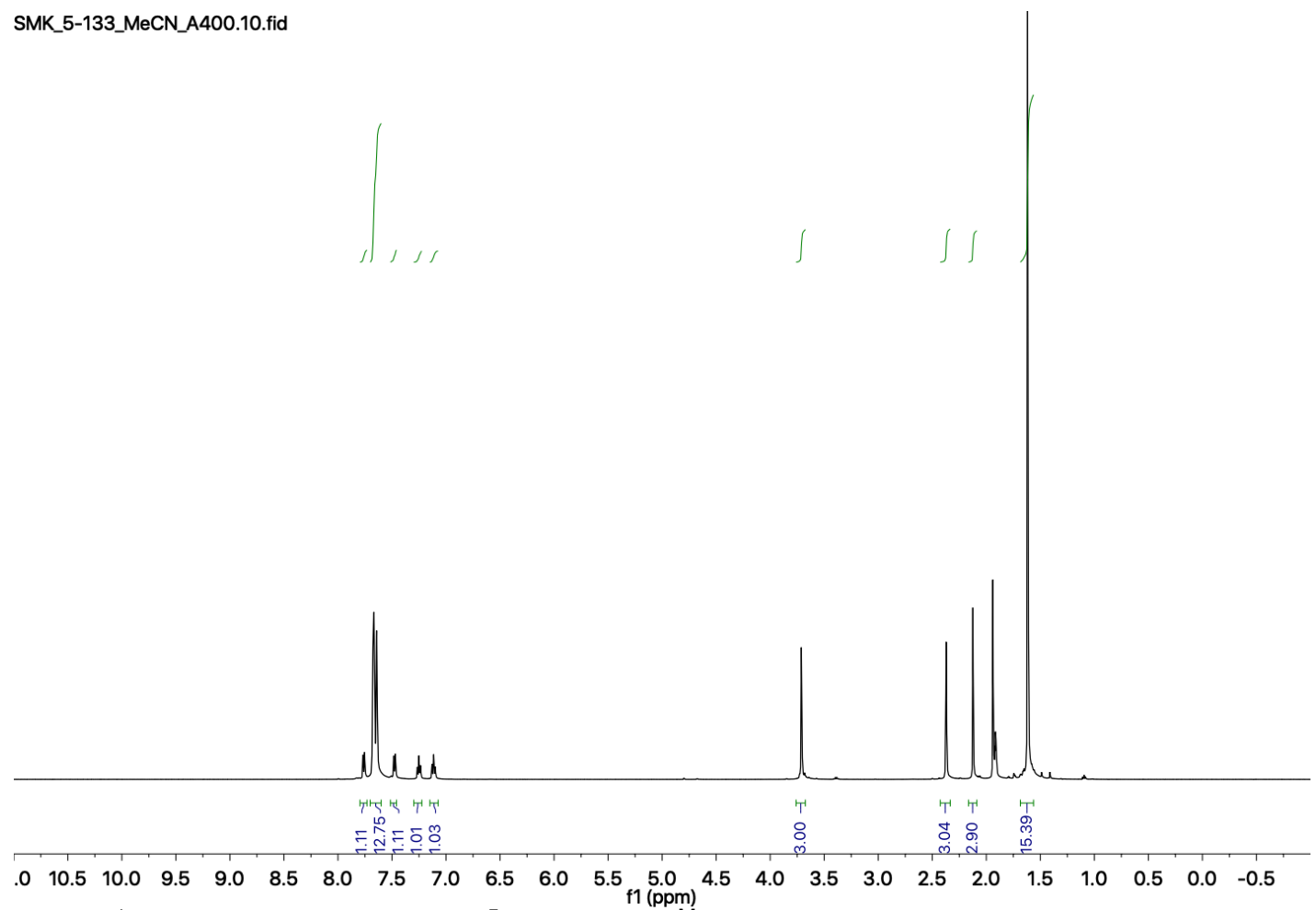

Figure S12. ${ }^{1} \mathrm{H}$ NMR spectrum of $\left[\left(\eta^{5}-\mathrm{C}_{5} \mathrm{Me}_{5}\right) \mathrm{Rh}\left({ }^{\mathrm{Me}} \mathrm{Phl}\right)(\mathrm{MeCN})\right]\left[\mathrm{BArF}{ }_{24}\right]$ in $\mathrm{MeCN}-d_{3}$. 


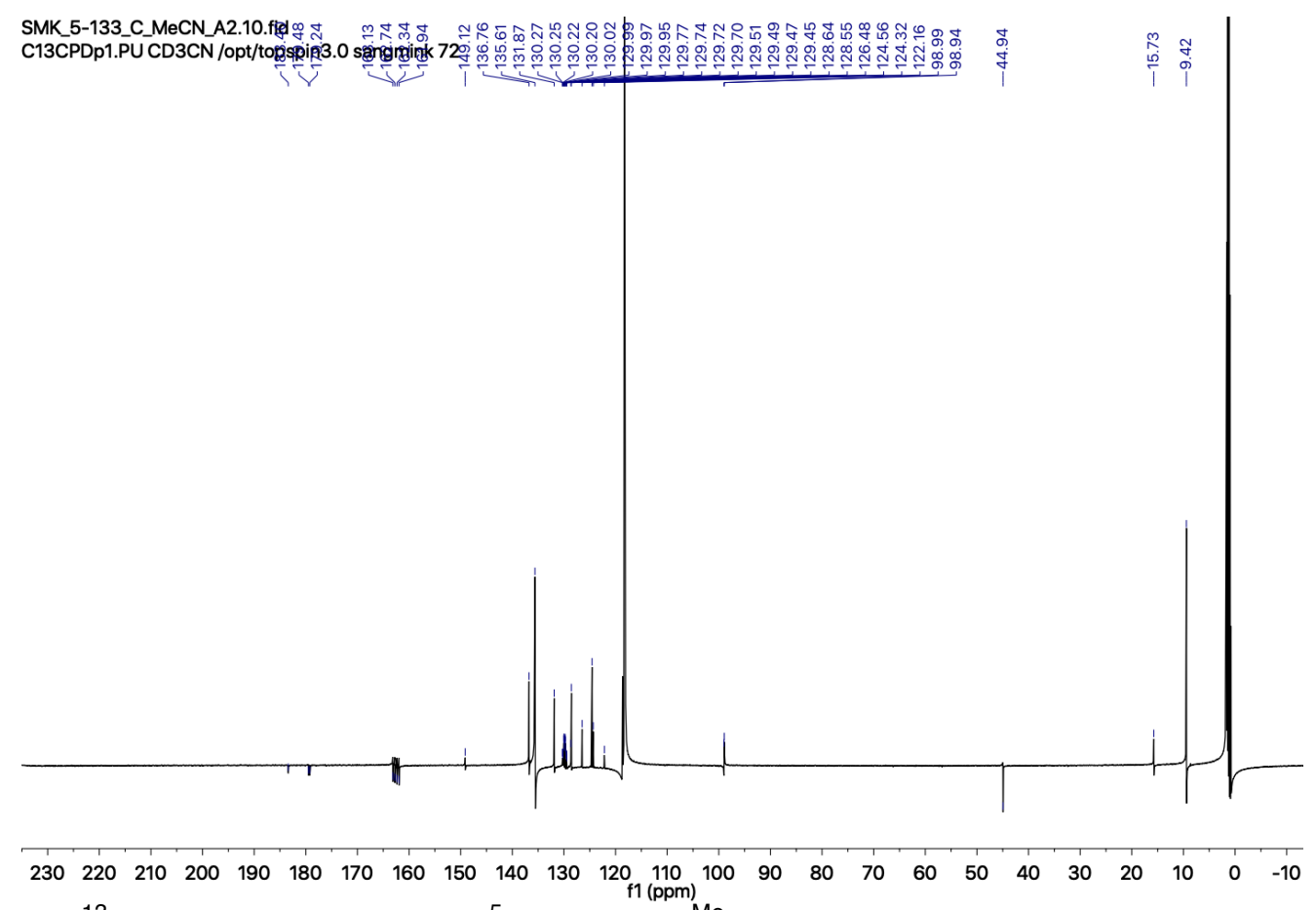

Figure S13. ${ }^{13} \mathrm{C}$ NMR spectrum of $\left[\left(\eta^{5}-\mathrm{C}_{5} \mathrm{Me}_{5}\right) \mathrm{Rh}\left({ }^{\mathrm{Me}} \mathrm{PhI}\right)(\mathrm{MeCN})\right]\left[\mathrm{BArF}{ }_{24}\right]$ in $\mathrm{MeCN}-d_{3}$.

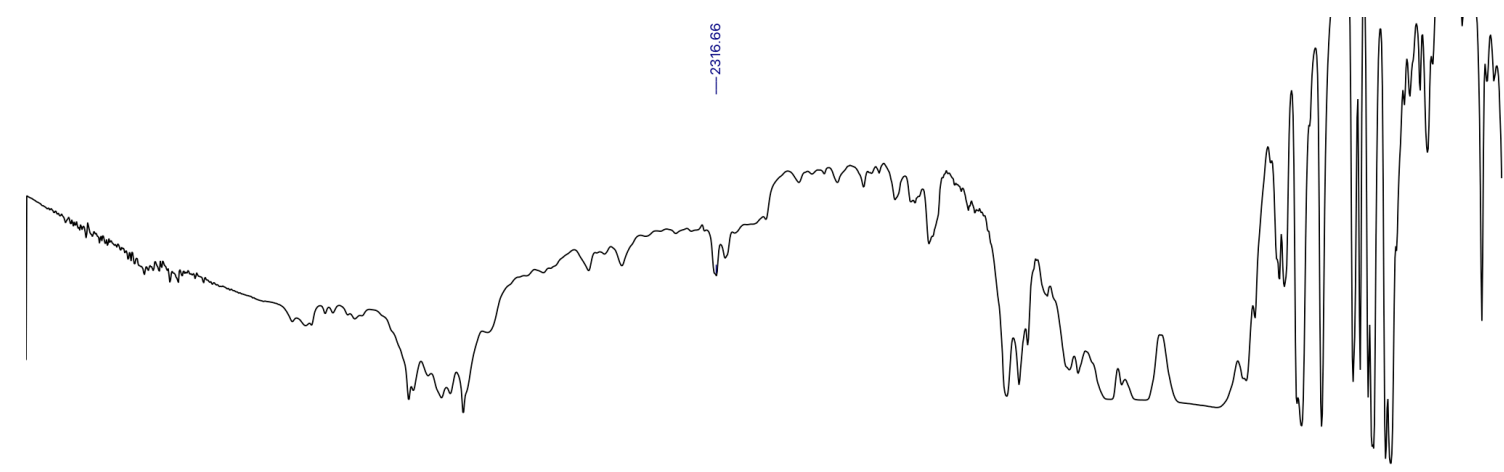

$00 \quad 3800 \quad 3600 \quad 3400 \quad 3200 \quad 3000 \quad 2800 \quad 2600 \quad 2400 \begin{array}{rllllllllllll}2200 & 2000 & 1800 & 1600 & 1400 & 1200 & 1000 & 800 & 600 & 4 C\end{array}$

Figure S14. IR spectrum of $\left[\left(\eta^{5}-\mathrm{C}_{5} \mathrm{Me}_{5}\right) \mathrm{Rh}\left({ }^{\mathrm{Me}} \mathrm{PhI}\right)(\mathrm{MeCN})\right][\mathrm{BArF} 24](\mathrm{KBr}$ pellet$)$. 


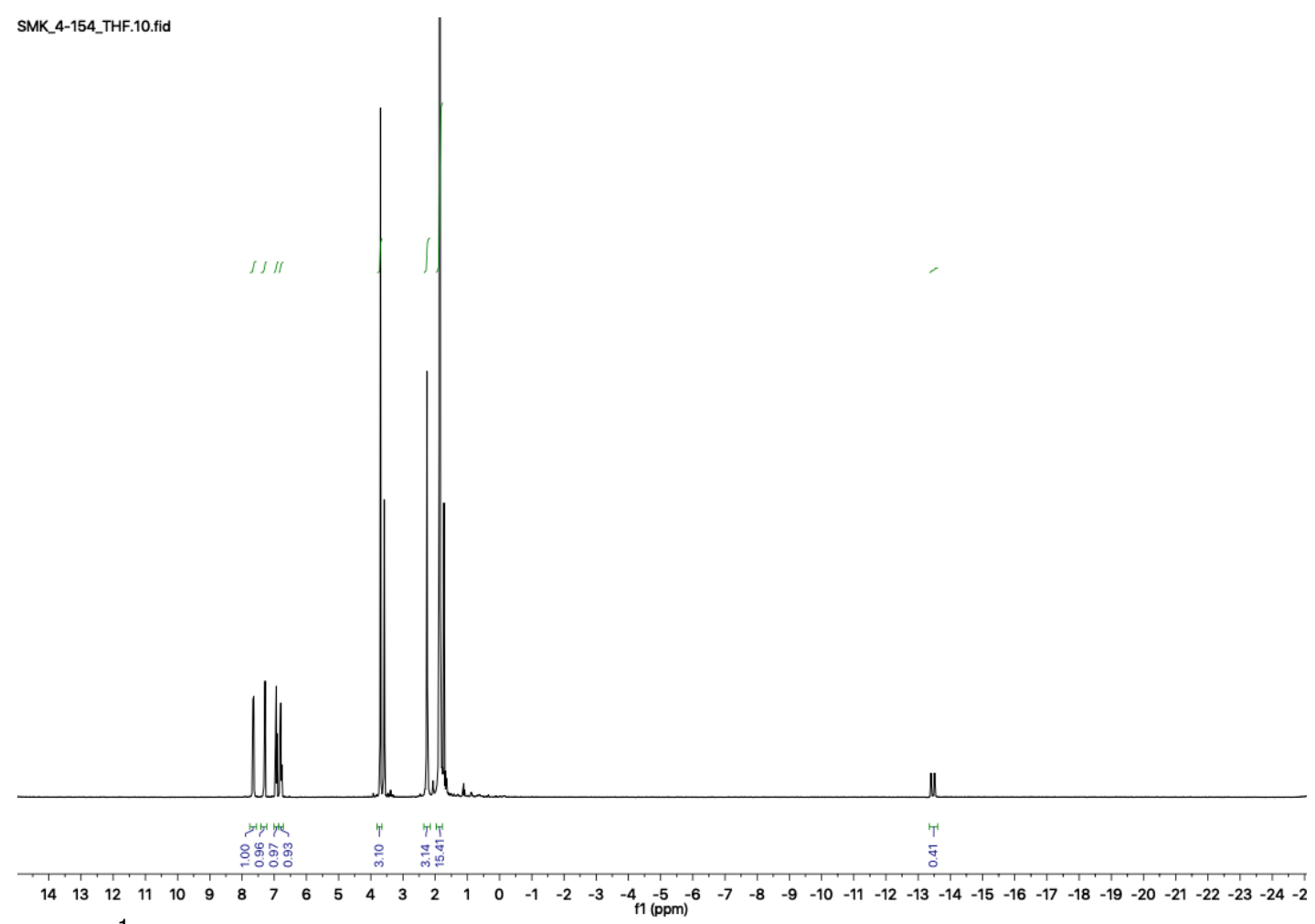

Figure S15. ${ }^{1} \mathrm{H}$ NMR spectrum of $2-d_{1}$ in THF- $d_{8}$.

SMK_4-155_deut_THF_A1.12 fid

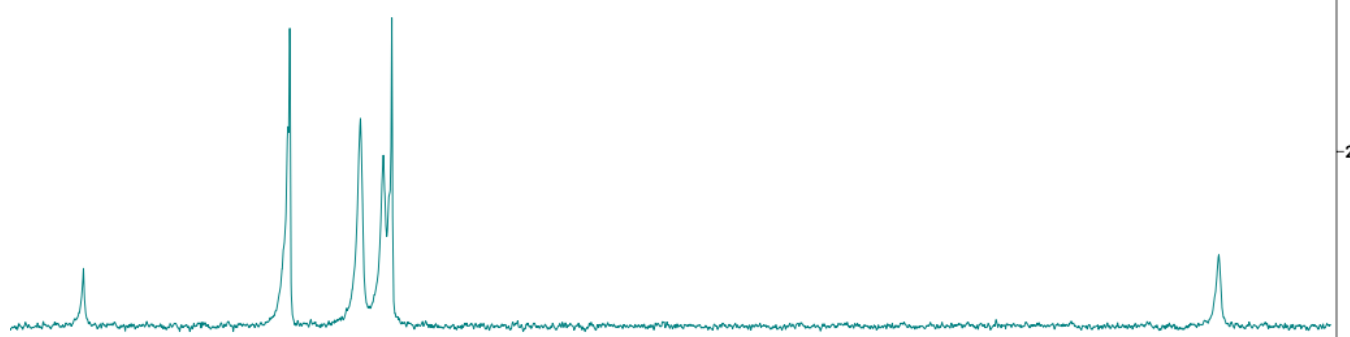

SMK_4-154_THF.10.fid
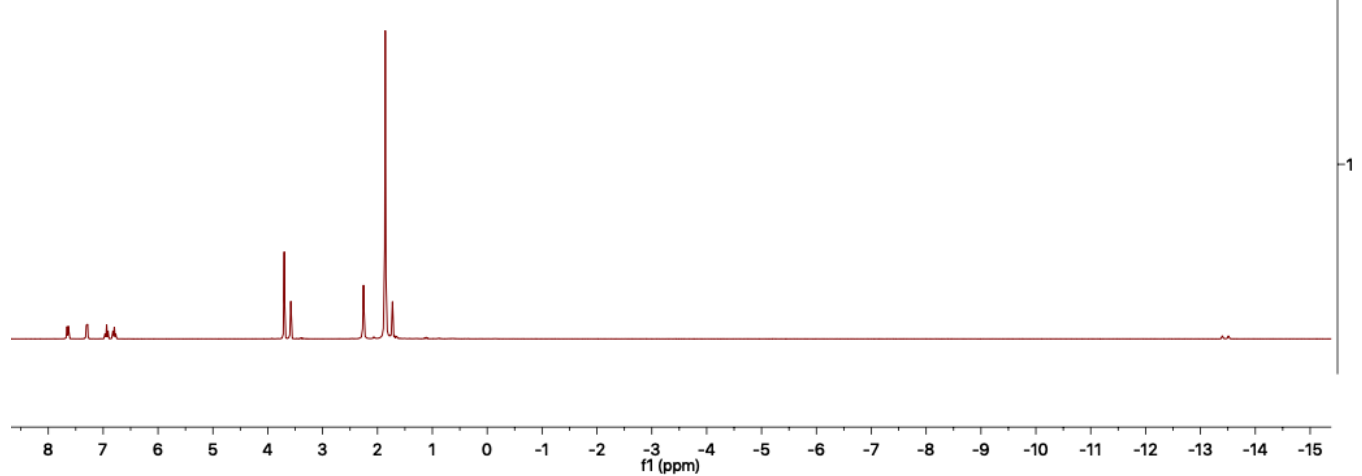

Figure S16. ${ }^{2} \mathrm{H}$ NMR spectrum of $2-d_{1}$ in THF- $d_{8}$ after being heated at $50{ }^{\circ} \mathrm{C}$ for $24 \mathrm{~h}$ (top). ${ }^{1} \mathrm{H}$ NMR spectrum of $2-d_{1}$ in $T H F-d_{8}$ for comparison (bottom). (Scheme $4 b$ ) 

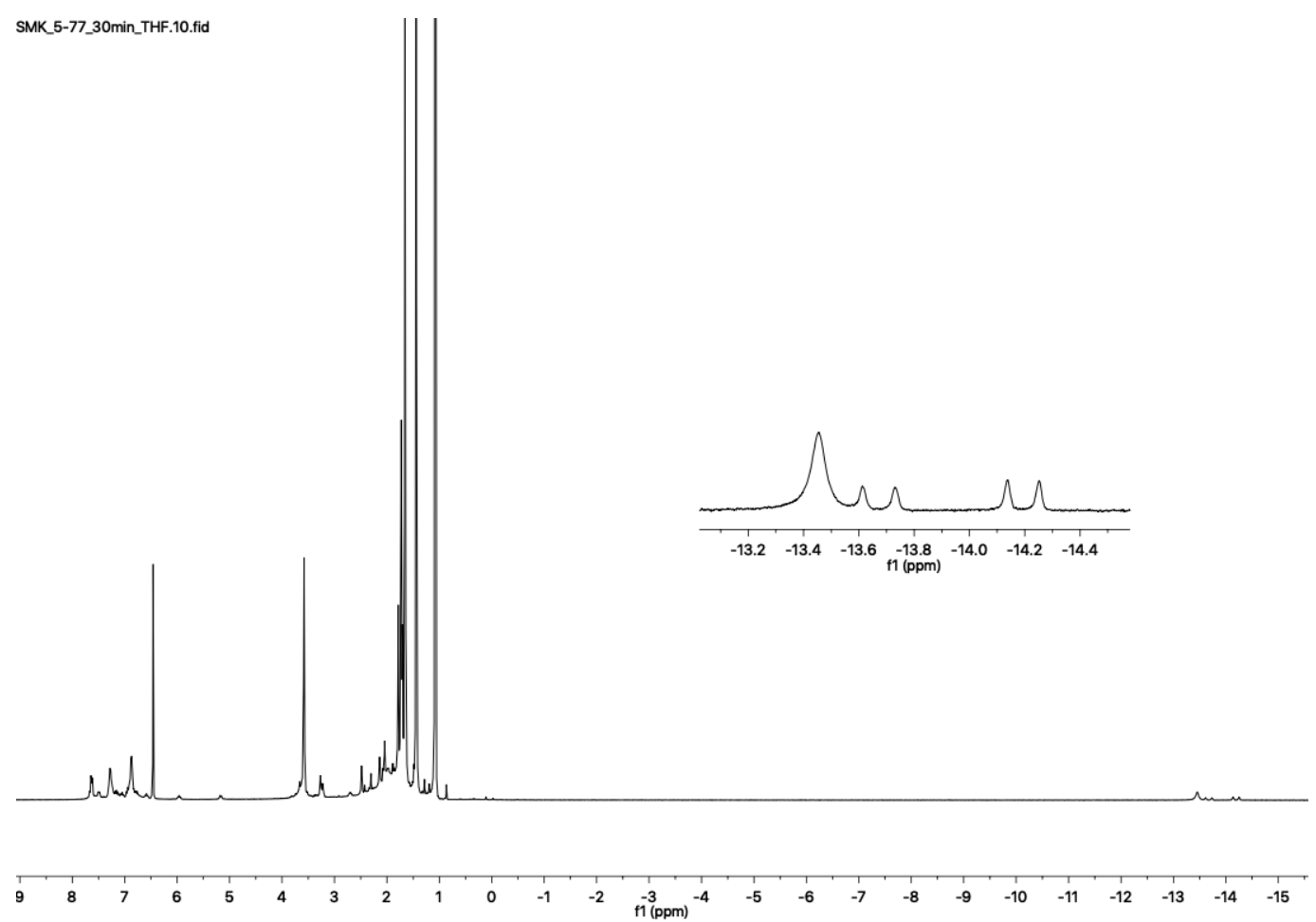

Figure S17. ${ }^{1} \mathrm{H}$ NMR spectrum of the crude mixture of 2 and TEMPO in THF- $d_{8}$.

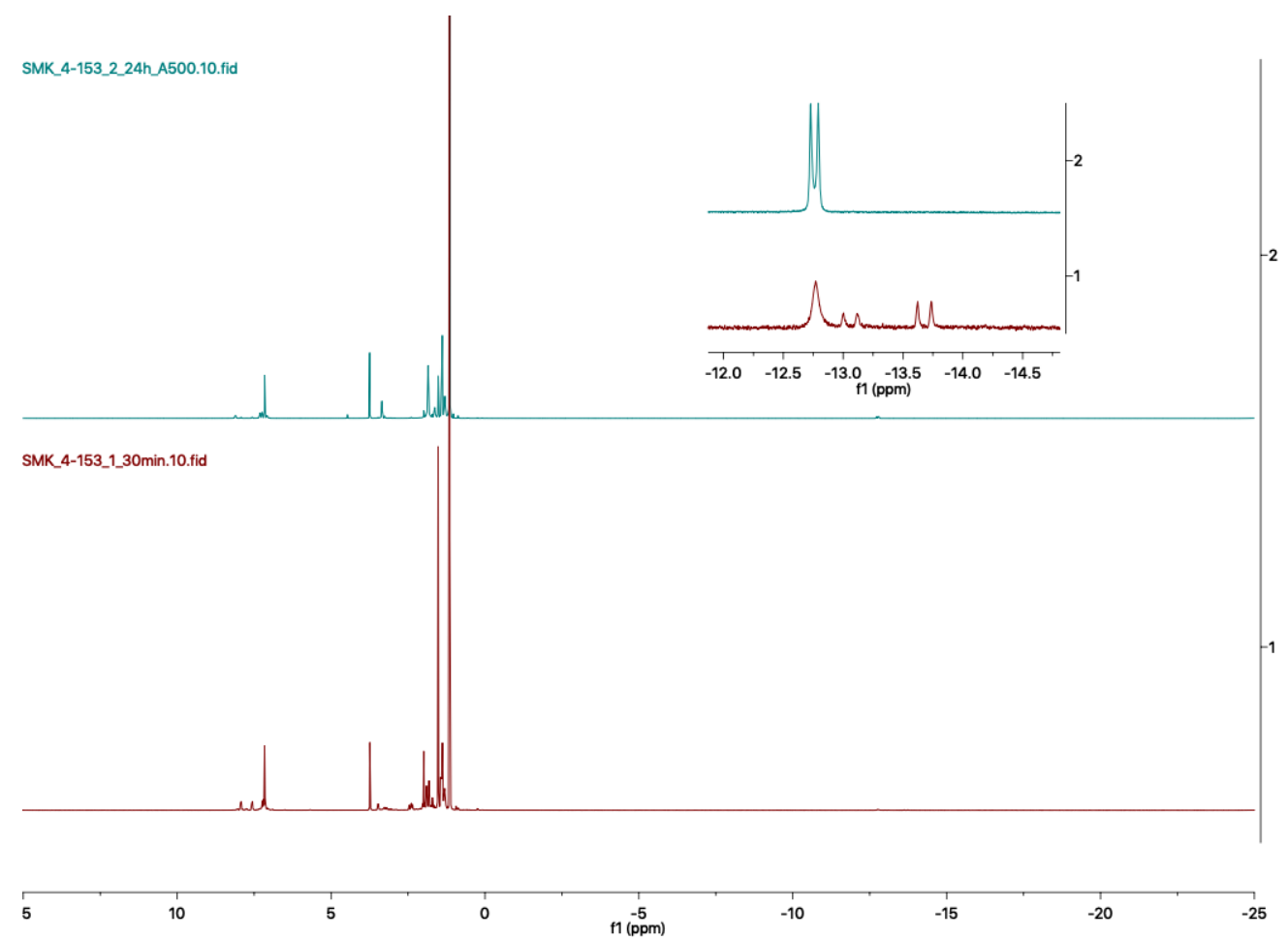

Figure S18. ${ }^{1} \mathrm{H}$ NMR spectra of the crude mixture of 2 and TEMPO in benzene- $d_{6}$ before (bottom) and after (top) addition of $4 \mathrm{~atm} \mathrm{H}_{2}$. (Scheme $5 \mathrm{a}$ ) 


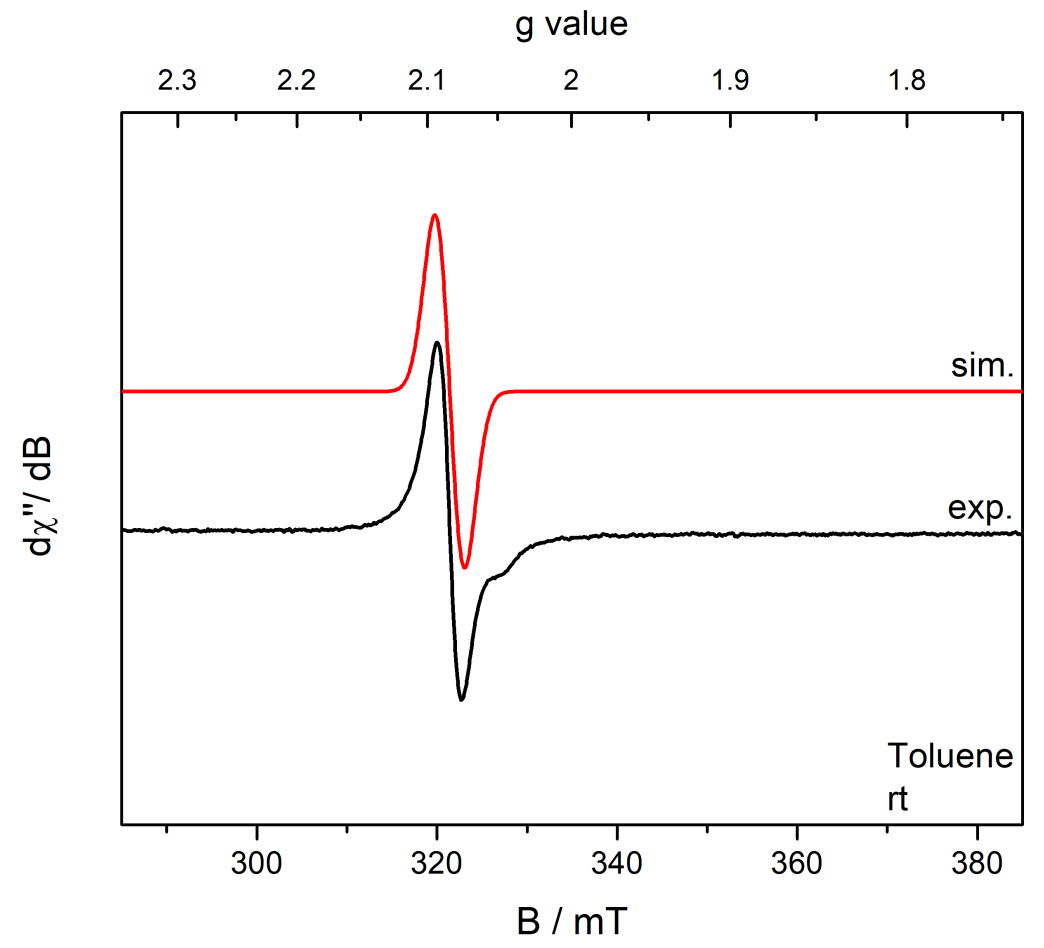

Figure S19. X-band EPR spectrum of the crude mixture of 2 and TEMPO recorded at room temperature in toluene (microwave frequency $=9.376 \mathrm{GHz}$, power $=2.0 \mathrm{~mW}$, power attenuation $=20.0 \mathrm{~dB}$, modulation amplitude $=4.000 \mathrm{G}$ ). Simulation $(\mathrm{red}): g_{\text {iso }}=2.084($ Scheme $5 \mathrm{~b})$

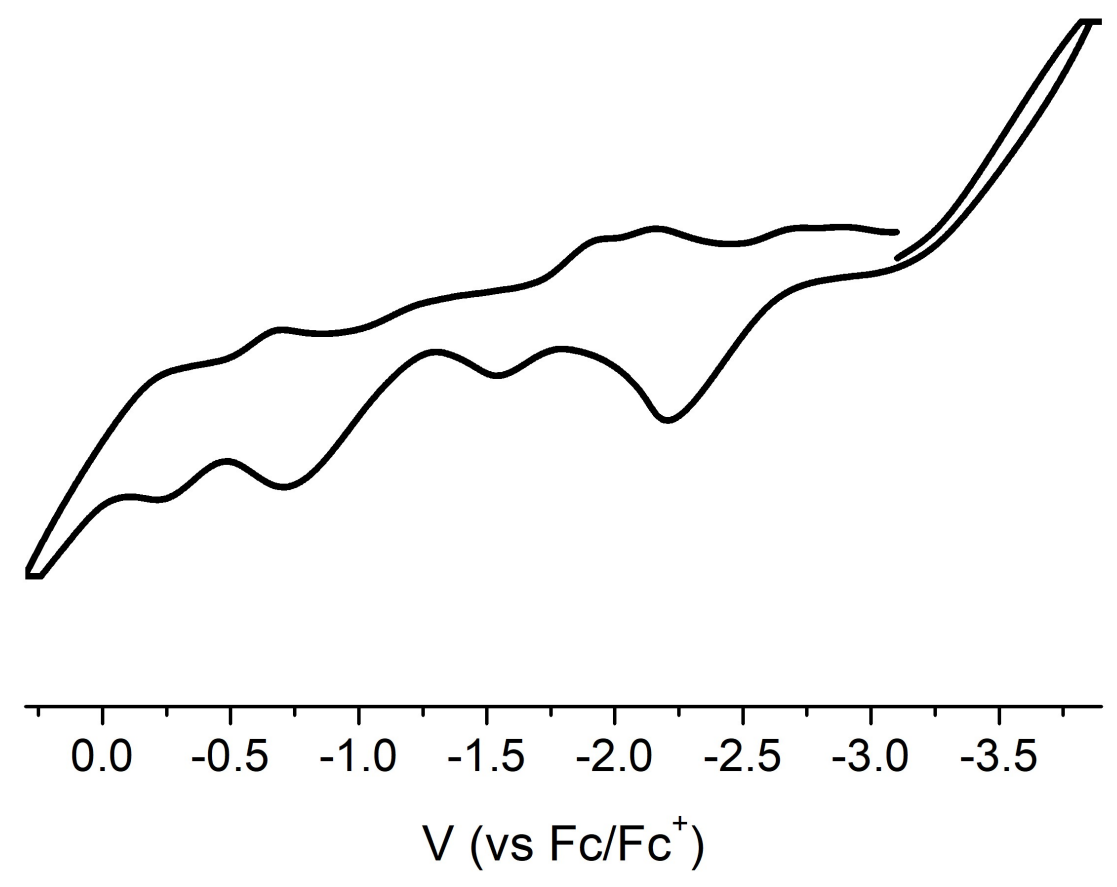

Figure S20. Cyclic voltammogram of the crude mixture of 2 and TEMPO using a glassy-carbon working electrode, a platinum wire counter electrode, a silver wire reference electrode, $0.1 \mathrm{M}$ $\left[{ }^{\mathrm{n}} \mathrm{Bu}_{4} \mathrm{~N}\right]\left[\mathrm{PF}_{6}\right]$, and a scan rate of $200 \mathrm{mV} / \mathrm{s}$ in THF at $295 \mathrm{~K}$ versus $\mathrm{Cp}_{2} \mathrm{Fe} / \mathrm{Cp}_{2} \mathrm{Fe}^{+}$. 


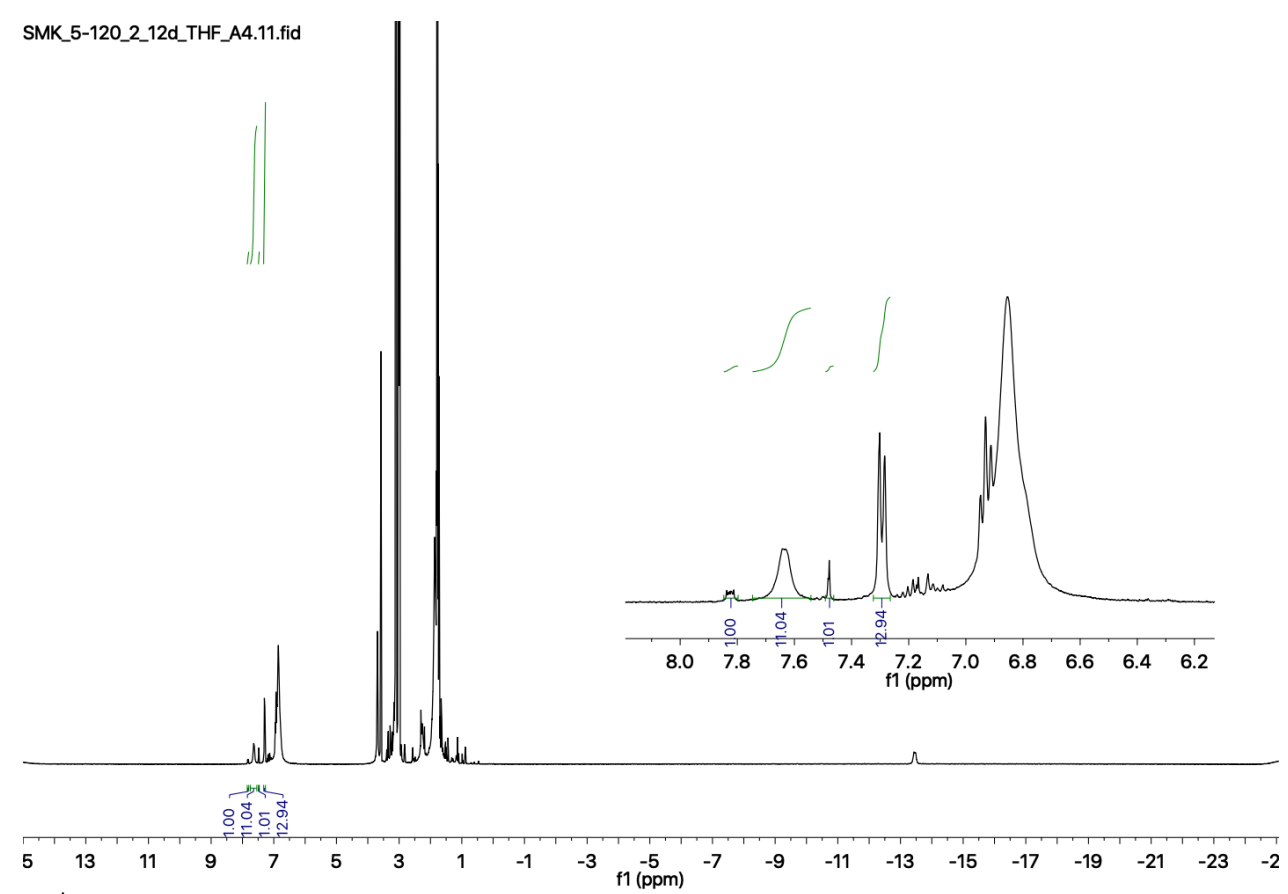

Figure S21. ${ }^{1} \mathrm{H}$ NMR spectrum of the crude mixture of 2 and TBD in THF- $d_{8}$. Broadened peaks are from 2 due to the dynamic equilibrium and new diamagnetic peaks formed are from the anionic species. (Scheme 5c)

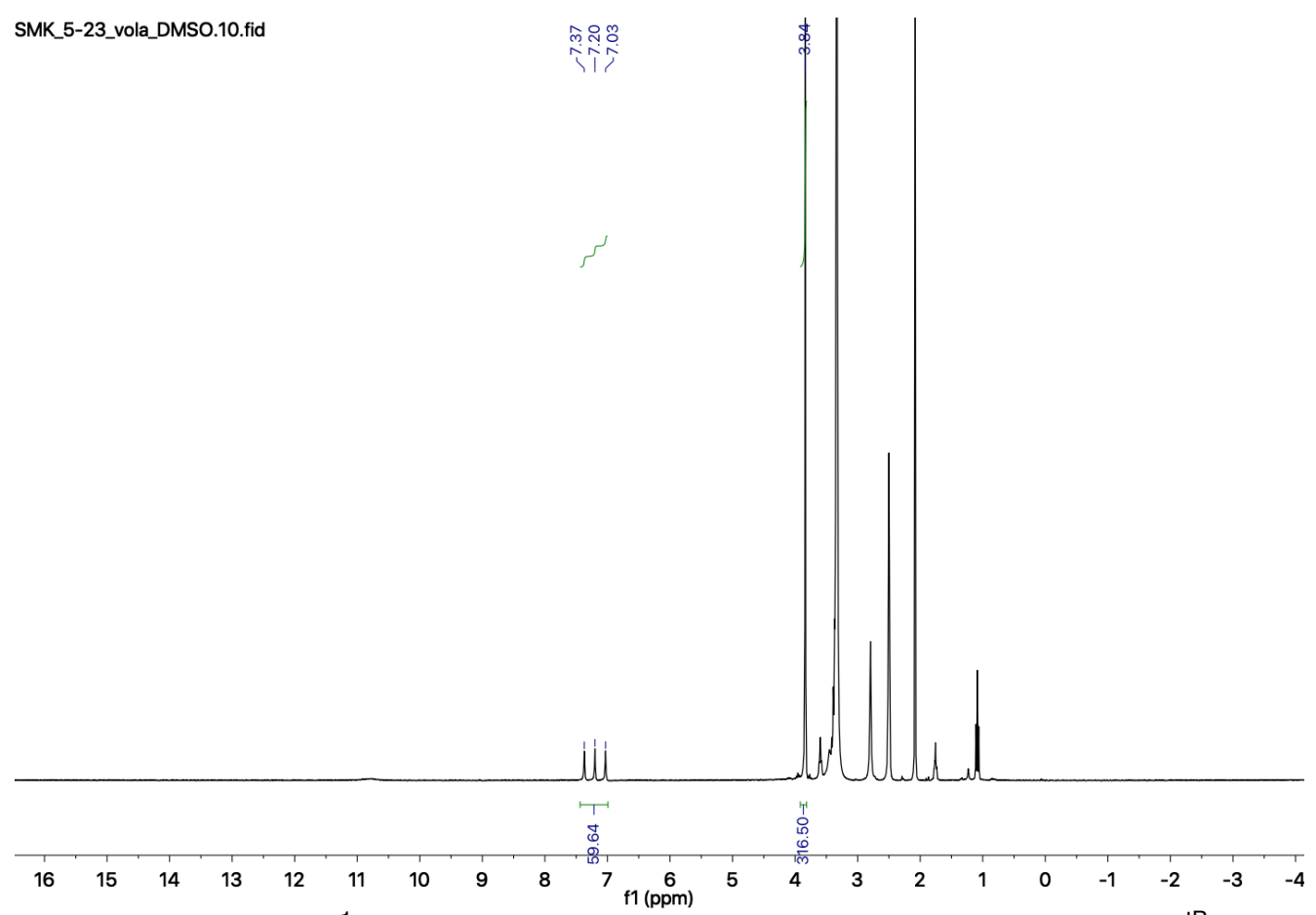

Figure S22. Representative ${ }^{1} \mathrm{H}$ NMR spectrum of $\mathrm{NH}_{4} \mathrm{Cl}$ from hydrogenation of ( $\left.{ }^{\mathrm{tBu}} \mathrm{Salen}\right) \mathrm{MnN}$ in DMSO $-d_{6}$. (5 $\mu \mathrm{mol}$ of 1,2-dichloroethane as an internal standard) (Main text Table 1 , entry 6 ) 


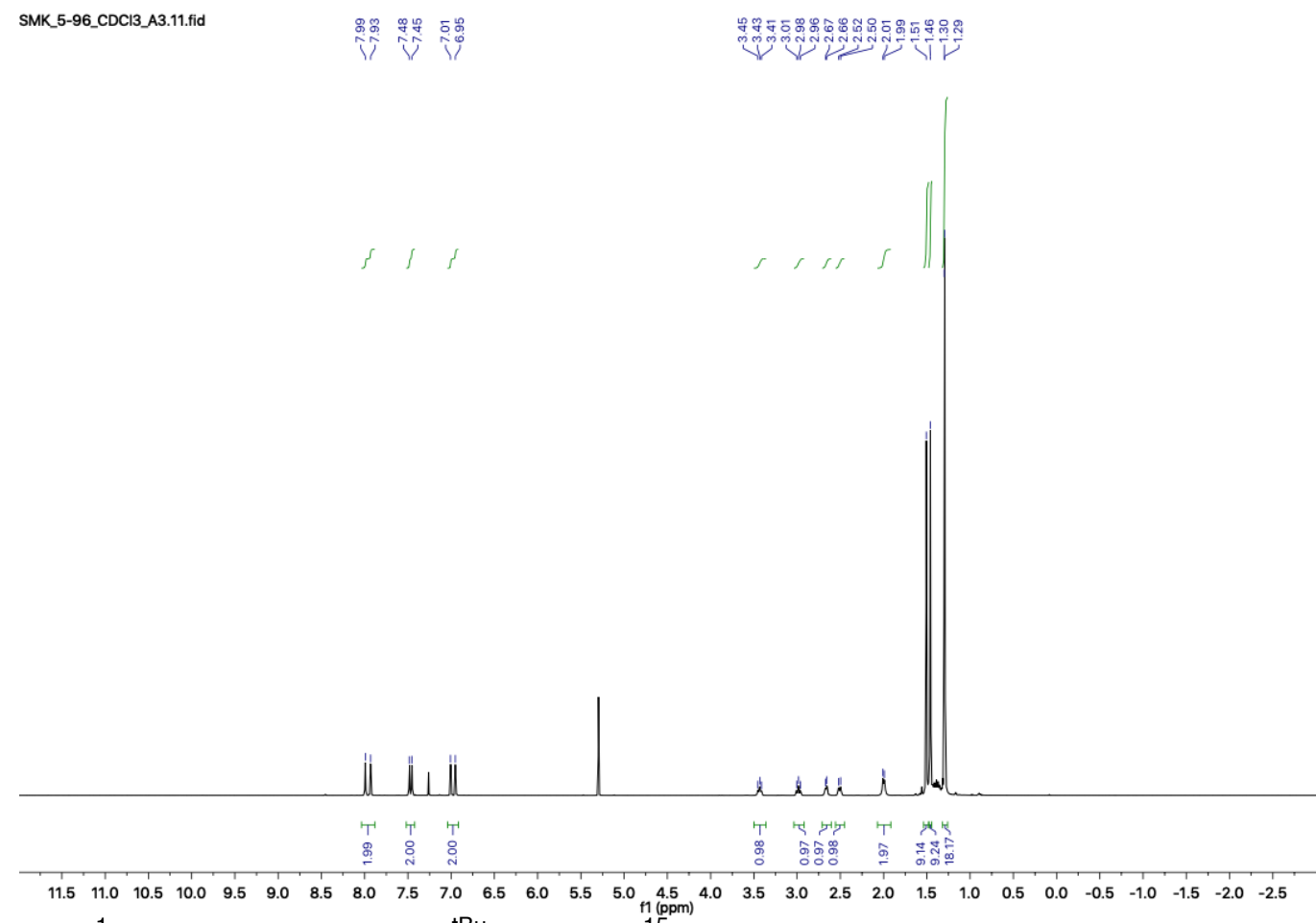

Figure S23. ${ }^{1} \mathrm{H}$ NMR spectrum of ( ${ }^{\mathrm{tBu}}$ Salen) $\mathrm{Mn}^{15} \mathrm{~N}$ in $\mathrm{CDCl}_{3}$.

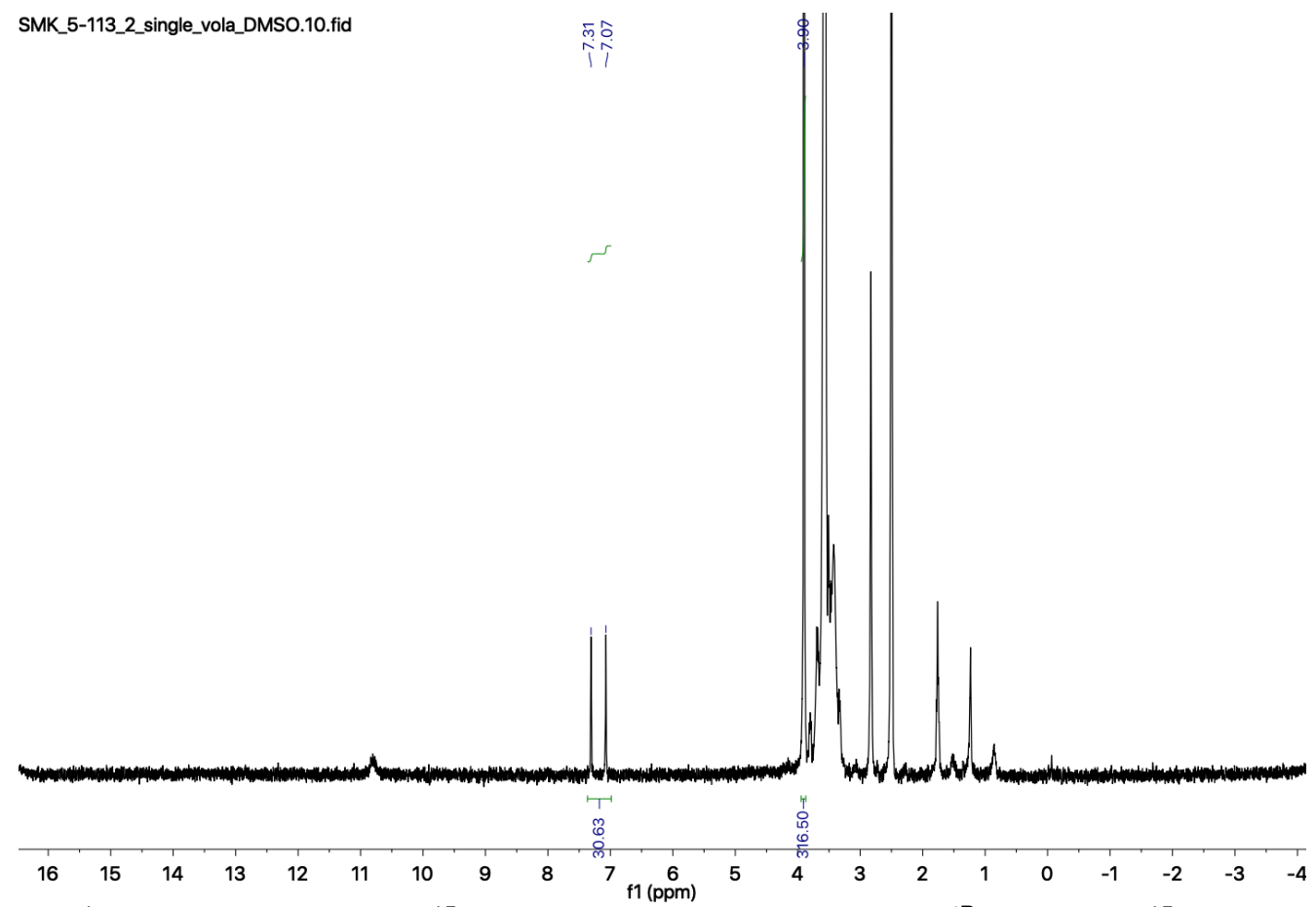

Figure S24. ${ }^{1} \mathrm{H}$ NMR spectrum of ${ }^{15} \mathrm{NH}_{4} \mathrm{Cl}$ from hydrogenation of ( $\left.{ }^{\mathrm{tBu}} \mathrm{Salen}\right) \mathrm{Mn}^{15} \mathrm{~N}$ in DMSO- $d_{6}$. (5 $\mu \mathrm{mol}$ of 1,2-dichloroethane as an internal standard) (Scheme 7a) 


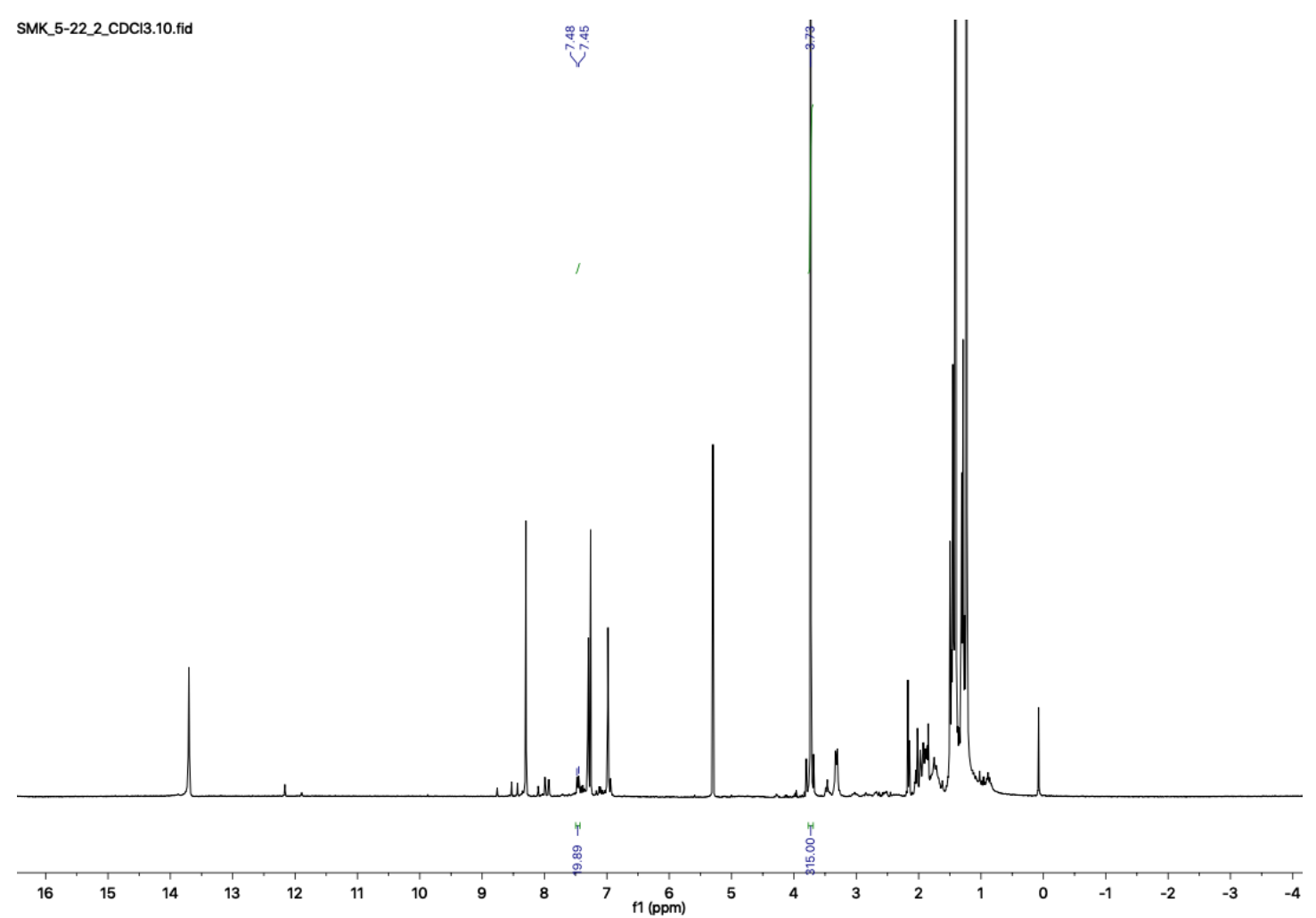

Figure S25. ${ }^{1} \mathrm{H}$ NMR spectrum of the recovered $\left({ }^{\mathrm{tBu}}\right.$ Salen)MnN from ( ${ }^{\mathrm{tBu}}$ Salen)MnCl which was generated in situ from the non-volatile reduced manganese residue after hydrogenation of ( ${ }^{\mathrm{tBu}}$ Salen) $\mathrm{MnN}$ in $\mathrm{CDCl}_{3}$. (5 $\mu \mathrm{mol}$ of 1,2-dichloroethane as an internal standard) (Scheme $7 \mathrm{~b}$ ) UV-Vis Spectroscopy

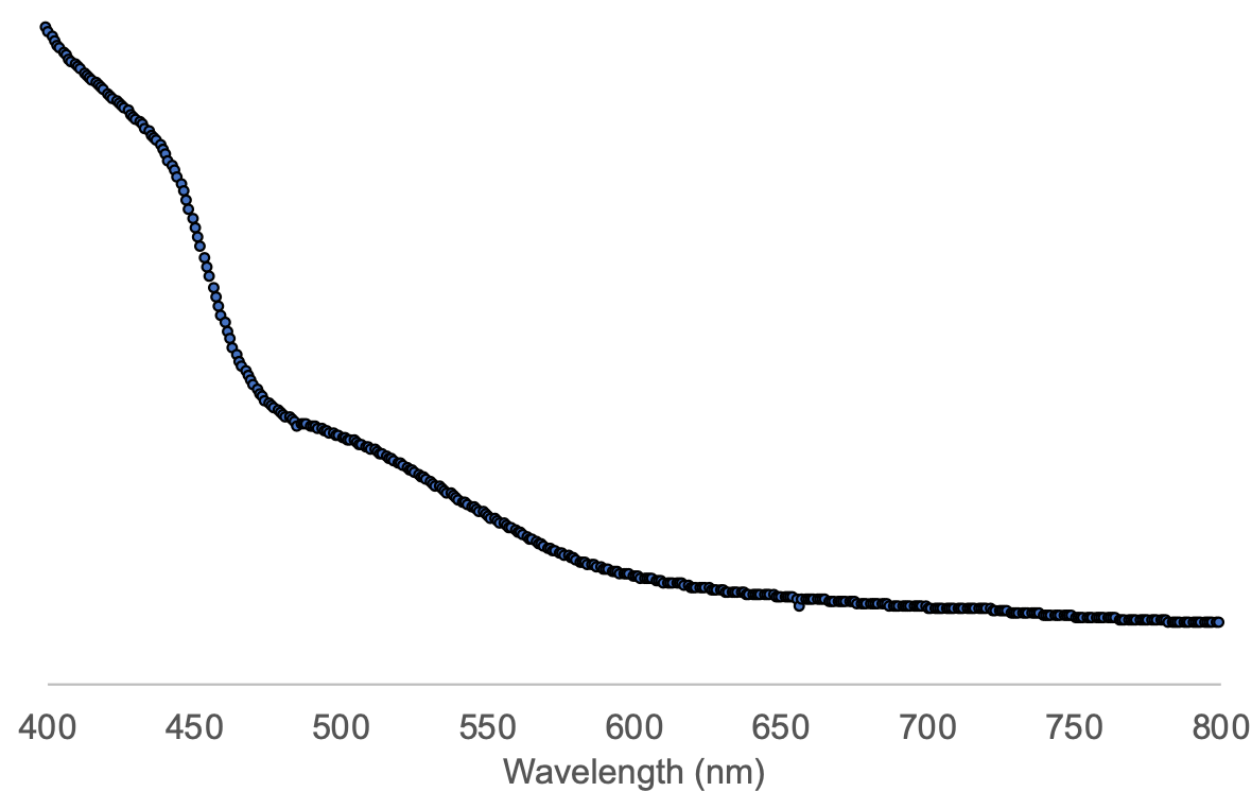

Figure S26. UV-Vis spectrum of in situ generated ( ${ }^{\mathrm{tBu}}$ Salen) $\mathrm{MnCl}$ in $\mathrm{CH}_{2} \mathrm{Cl}_{2}$. (Scheme $7 \mathrm{~b}$ ) 


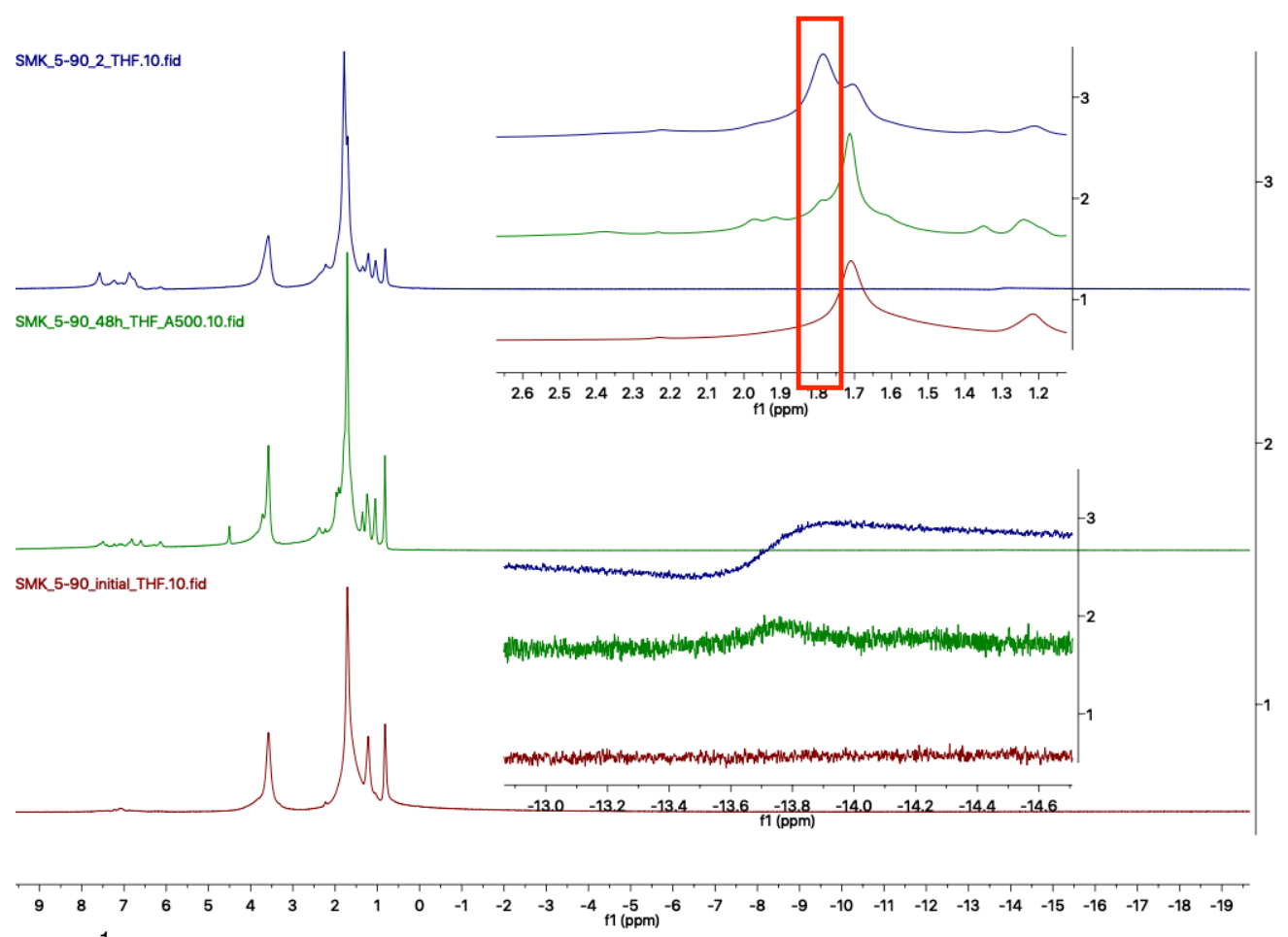

Figure S27. ${ }^{1} \mathrm{H}$ NMR spectra of the crude mixture of 1 equivalent 2 and 2 equivalents of ( ${ }^{\text {tBu }}$ Salen)MnN in THF- $d_{8}$ before (bottom) and after (middle) addition of 4 atm $\mathrm{H}_{2}$. Additional 2 equivalents of 2 were spiked to the middle to identify the $\left(\eta^{5}-\mathrm{C}_{5} \mathrm{Me}_{5}\right)$ and hydride peaks of 2 (top). The red box shows the increase of $\left(\eta^{5}-\mathrm{C}_{5} \mathrm{Me}_{5}\right)$ signal after $\mathrm{H}_{2}$ addition. (Scheme 8a)

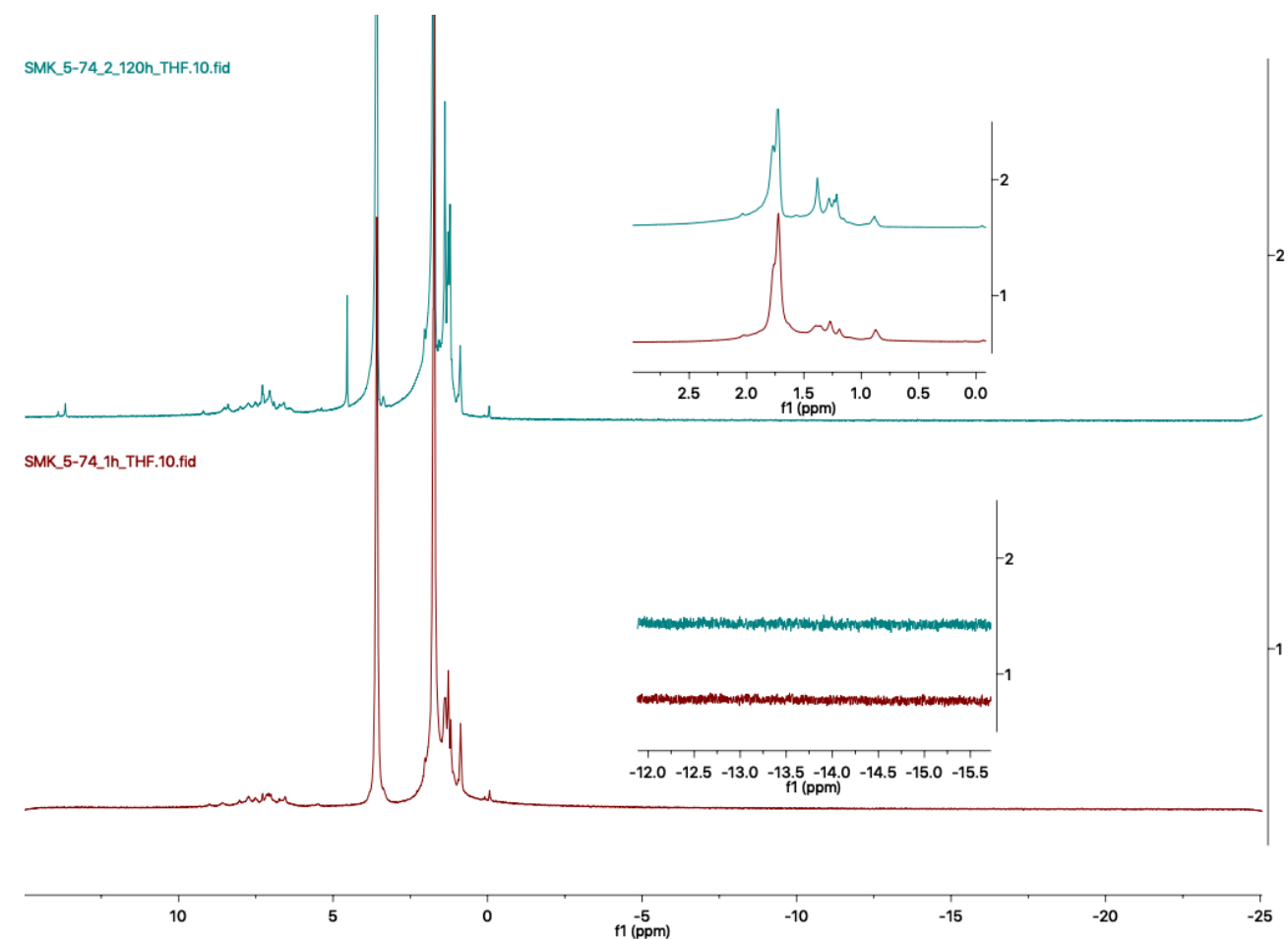

Figure S28. ${ }^{1} \mathrm{H}$ NMR spectra of the crude mixture of 1 equivalent 1 and 2 equivalents ( ${ }^{\mathrm{tBu}}$ Salen)MnN in THF- $d_{8}$ before (bottom) and after (top) addition of $4 \mathrm{~atm} \mathrm{H}_{2}$. No signal for $\left(\eta^{5}\right.$ $\mathrm{C}_{5} \mathrm{Me}_{5}$ ) or the hydride of 1 was observed. (Scheme $8 \mathrm{~b}$ ) 


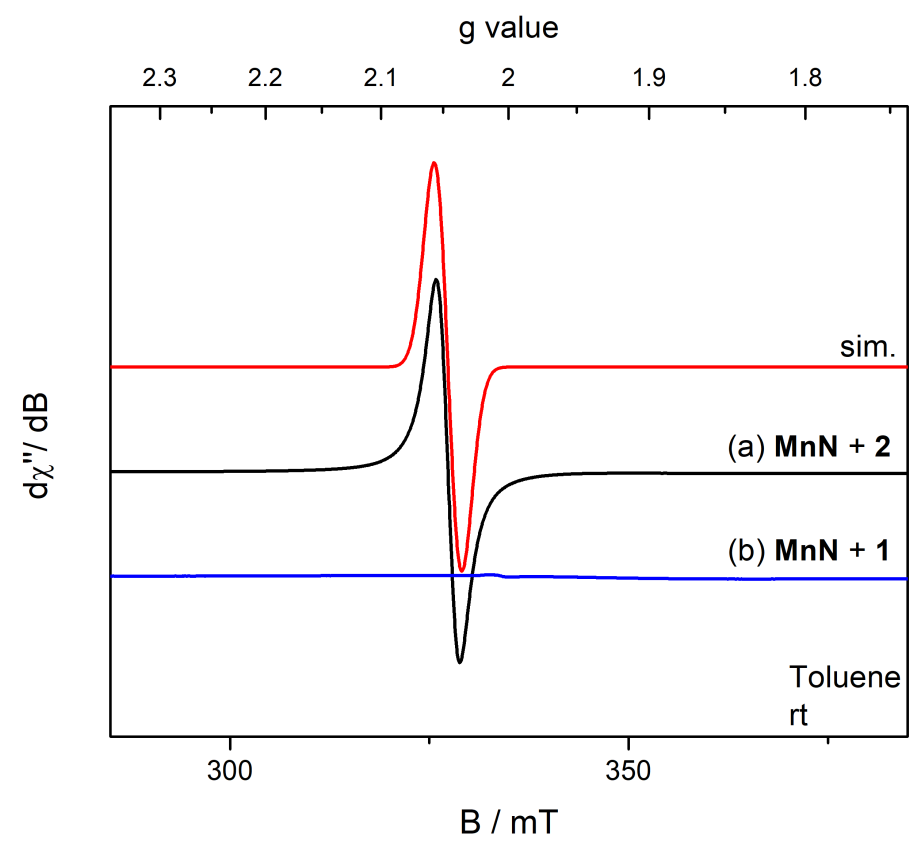

Figure S29. X-band EPR spectra of (a) the crude mixture of 1 equiv 2 and 2 equiv ( ${ }^{\text {tBu }}$ Salen) $\mathrm{MnN}$ recorded at room temperature in toluene (black) (microwave frequency $=9.376$ $\mathrm{GHz}$, power $=2.0 \mathrm{~mW}$, power attenuation $=20.0 \mathrm{~dB}$, modulation amplitude $=4.000 \mathrm{G}$ ) and $(\mathrm{b})$ the crude mixture of 1 equiv 1 and 2 equiv ( $\left.{ }^{\mathrm{Bu}} \mathrm{Salen}\right) \mathrm{MnN}$ (blue) (microwave frequency $=9.379$ $\mathrm{GHz}$, power $=2.0 \mathrm{~mW}$, power attenuation $=20.0 \mathrm{~dB}$, modulation amplitude $=4.000 \mathrm{G}$ ). Simulation (red): $g_{\text {iso }}=2.047$. Concentration of the samples were $0.02 \mathrm{M}$. (Scheme 8c)

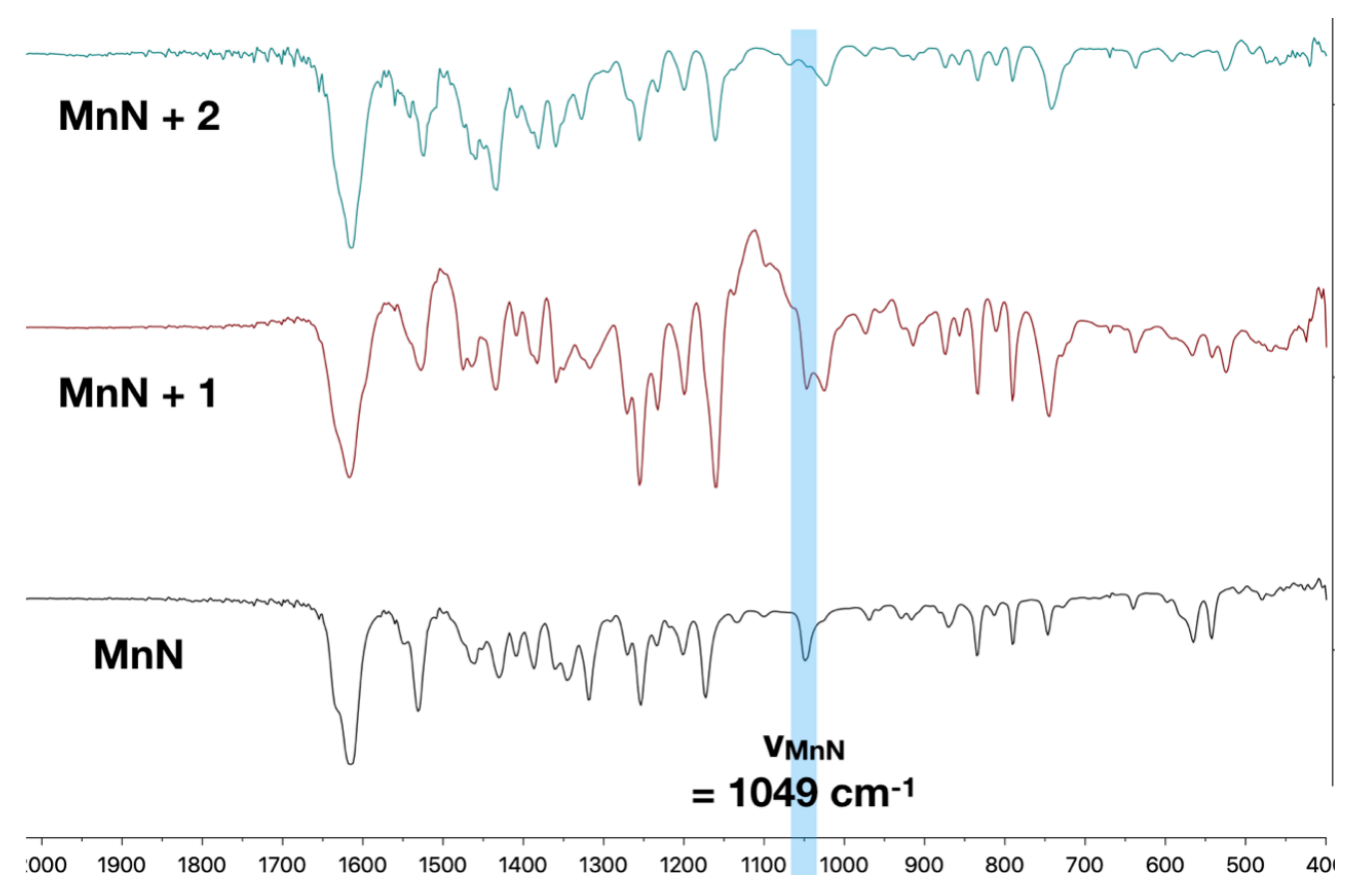

Figure S30. IR spectra of authentic sample of $\mathrm{MnN}$ (bottom), the crude mixture of 1 equiv 1 and 2 equiv $\mathrm{MnN}$ prepared in THF (middle) and the crude mixture of 1 equiv 2 and 2 equiv $\mathrm{MnN}$ prepared in THF in $\mathrm{KBr}$ pellets. (Scheme 8a,b) 


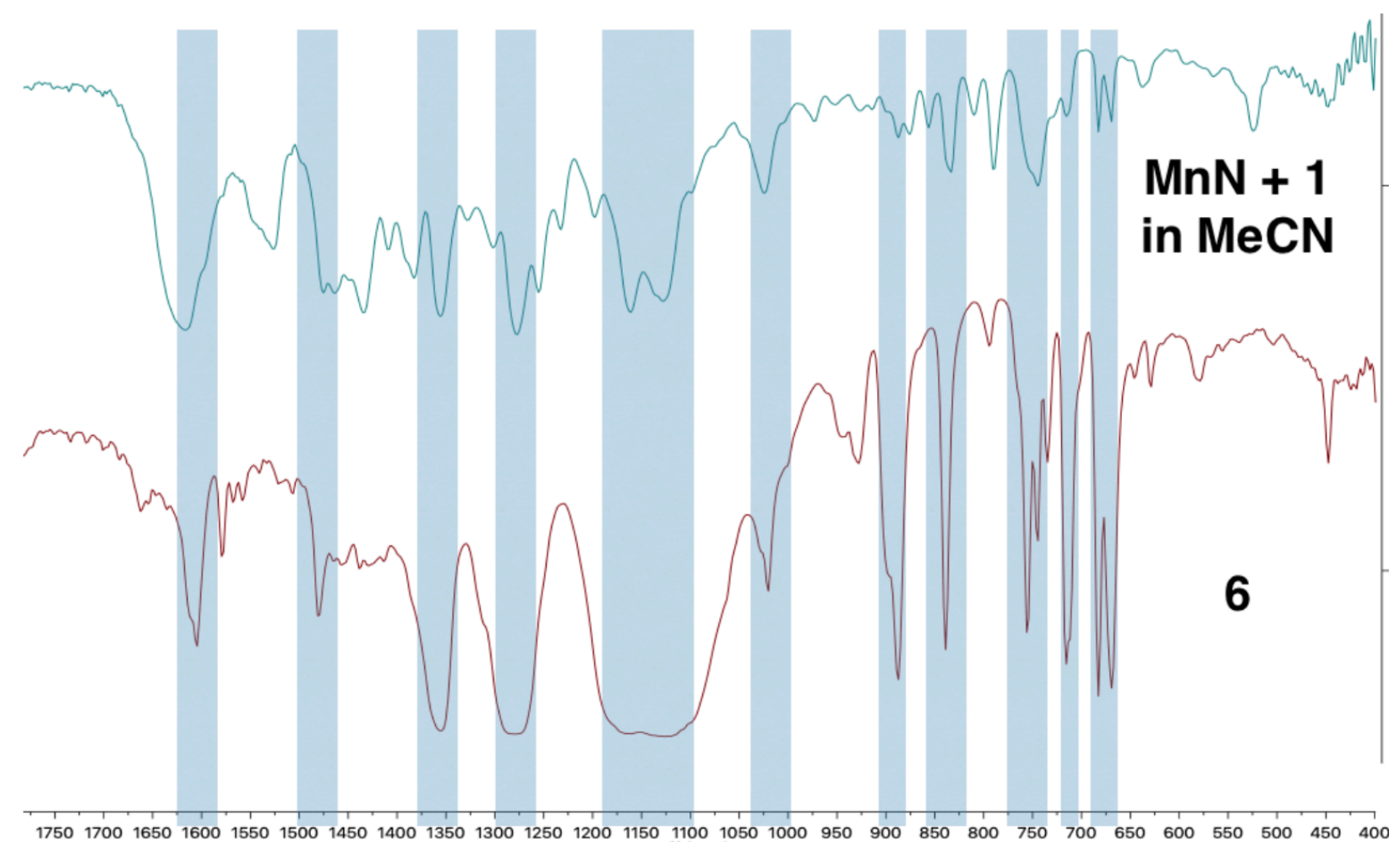

Figure S31. IR spectra of authentic sample of 6 (bottom), the crude mixture of 1 equiv 1 and 1 equiv $\mathrm{MnN}$ (top) prepared in MeCN (KBr pellets). All the signals of 6 appear in the IR spectrum of the crude reaction mixture highlighted in the blue boxes. (Scheme 9)
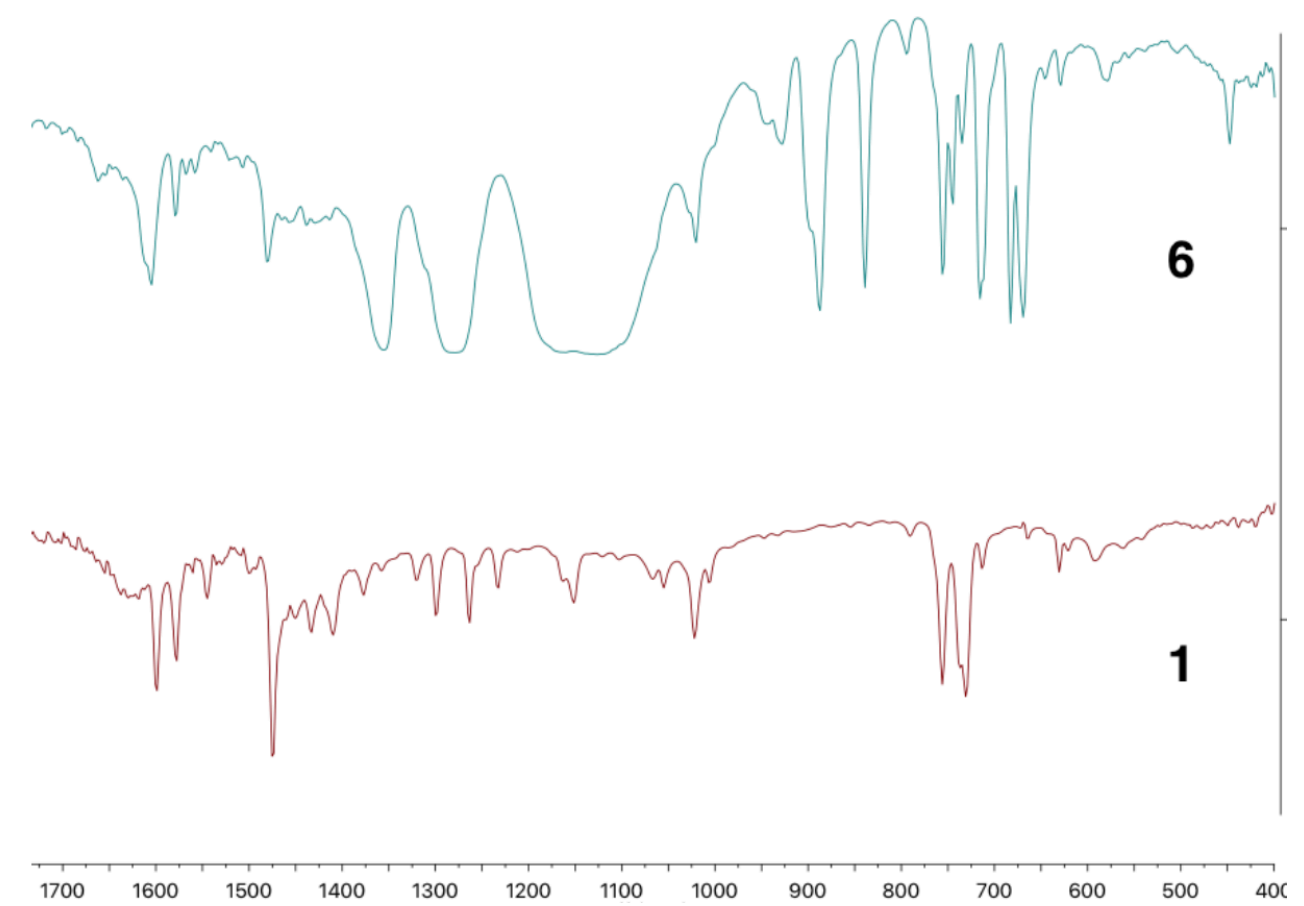

Figure S32. IR spectra of authentic sample of 1 (bottom) and 6 for comparison of the fingerprint region. 


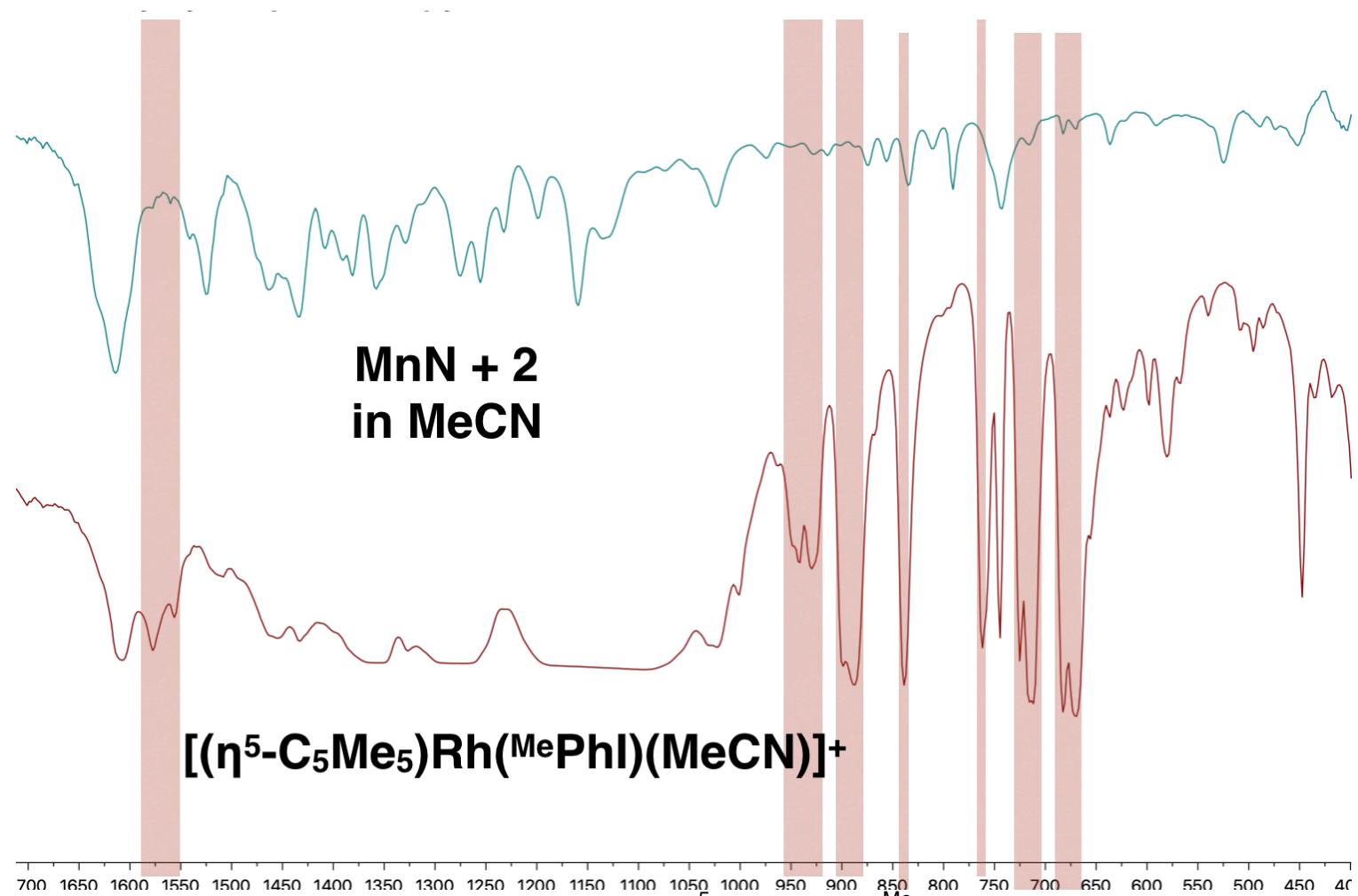

Figure S33. IR spectra of authentic sample of $\left[\left(\eta^{5}-\mathrm{C}_{5} \mathrm{Me}_{5}\right) \mathrm{Rh}\left({ }^{\mathrm{Me}} \mathrm{Phl}\right)(\mathrm{MeCN})\right]\left[\mathrm{BArF}_{24}\right]$ (bottom), the crude mixture of 1 equiv 2 and 1 equiv $\mathrm{MnN}$ (top) prepared in MeCN (KBr pellets). All the signals of $\left[\left(\eta^{5}-\mathrm{C}_{5} \mathrm{Me}_{5}\right) \mathrm{Rh}\left({ }^{\mathrm{Me}} \mathrm{PhI}\right)(\mathrm{MeCN})\right]\left[\mathrm{BArF}_{24}\right]$ does not appear in the IR spectrum of the crude reaction mixture highlighted in the red boxes.

$\mathrm{g}$ value

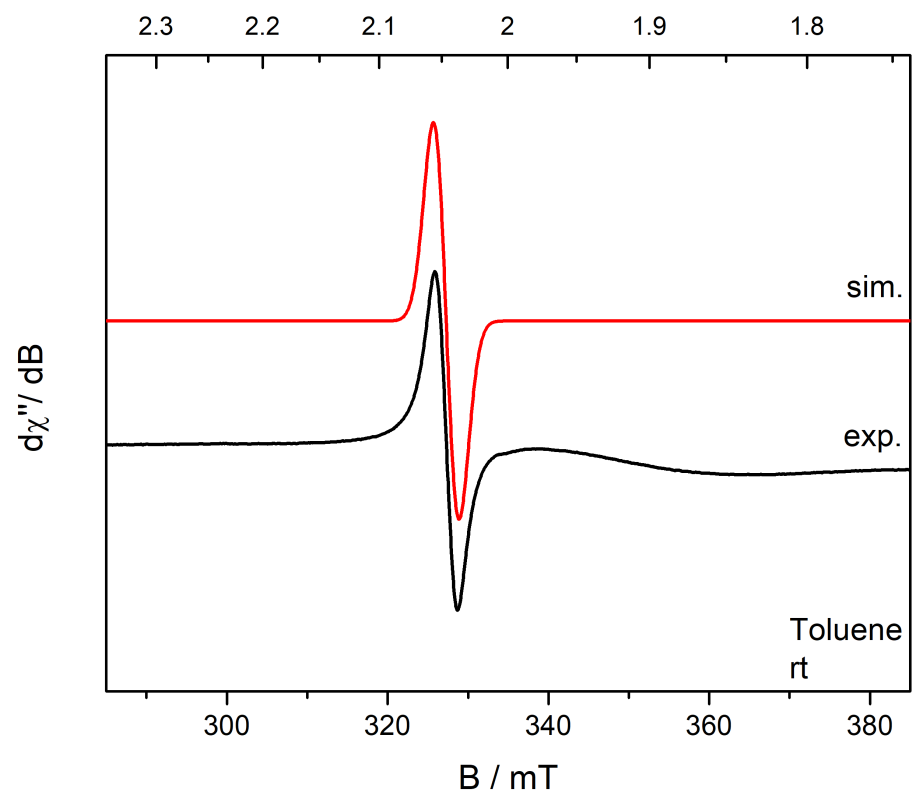

Figure S34. X-band EPR spectrum of the crude mixture of 1 and $\mathrm{MnN}$ prepared in benzene recorded at room temperature in toluene (microwave frequency $=9.379 \mathrm{GHz}$, power $=2.0 \mathrm{~mW}$, power attenuation $=20.0 \mathrm{~dB}$, modulation amplitude $=4.000 \mathrm{G}$ ). Simulation $(\mathrm{red}): g_{\text {iso }}=2.047$ (Scheme 10a) 


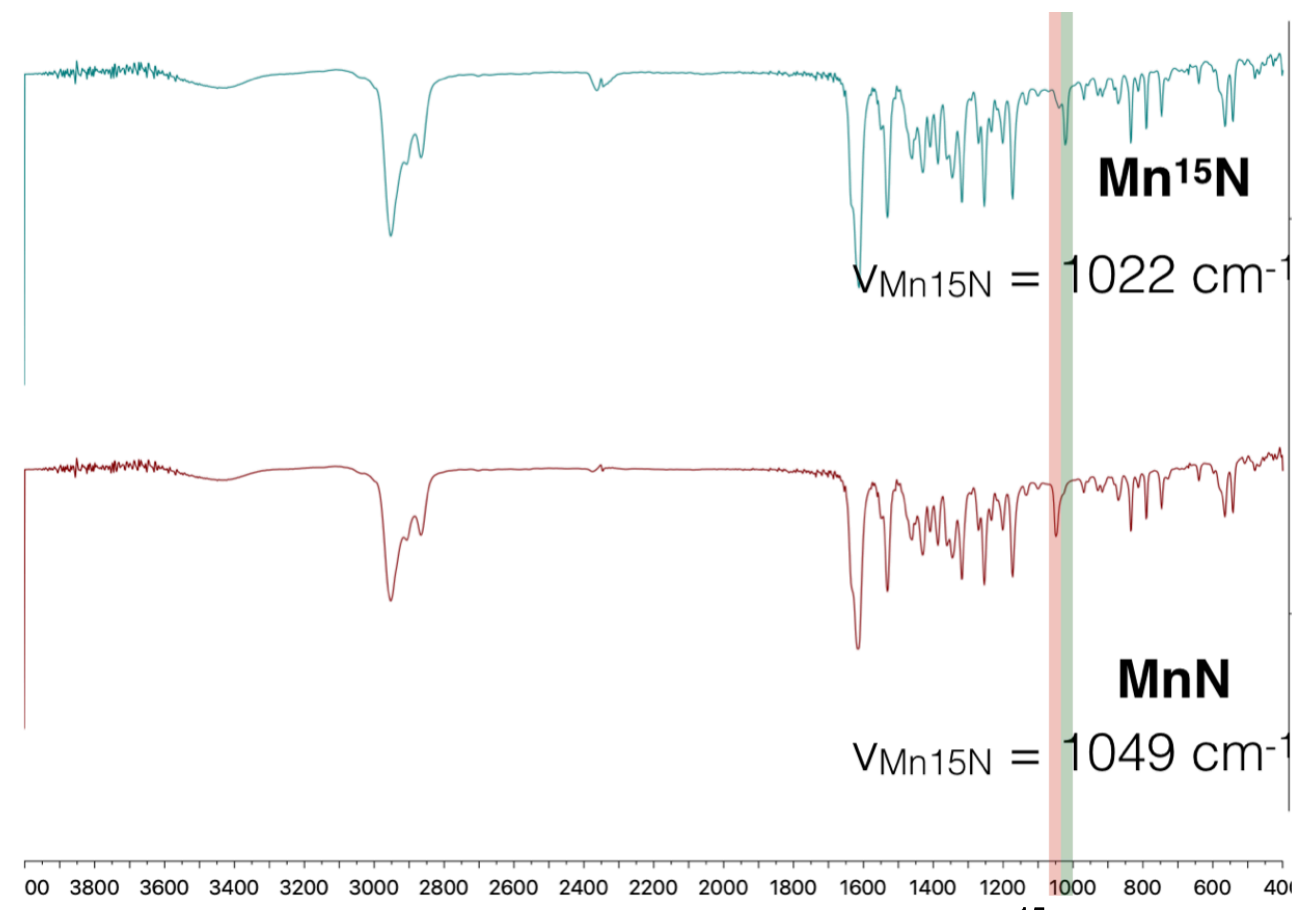

Figure S35. IR spectra of authentic sample of $\mathrm{MnN}$ (bottom) and $\mathrm{Mn}^{15} \mathrm{~N}$ (top) in $\mathrm{KBr}$ pellets. 


\section{Crystallographic Data}

\section{Compound $\left(\eta^{5}-\mathrm{C}_{5} \mathrm{Me}_{5}\right) \mathrm{Rh}\left({ }^{\mathrm{Me}} \mathrm{PhI}\right) \mathrm{Cl}$ :}

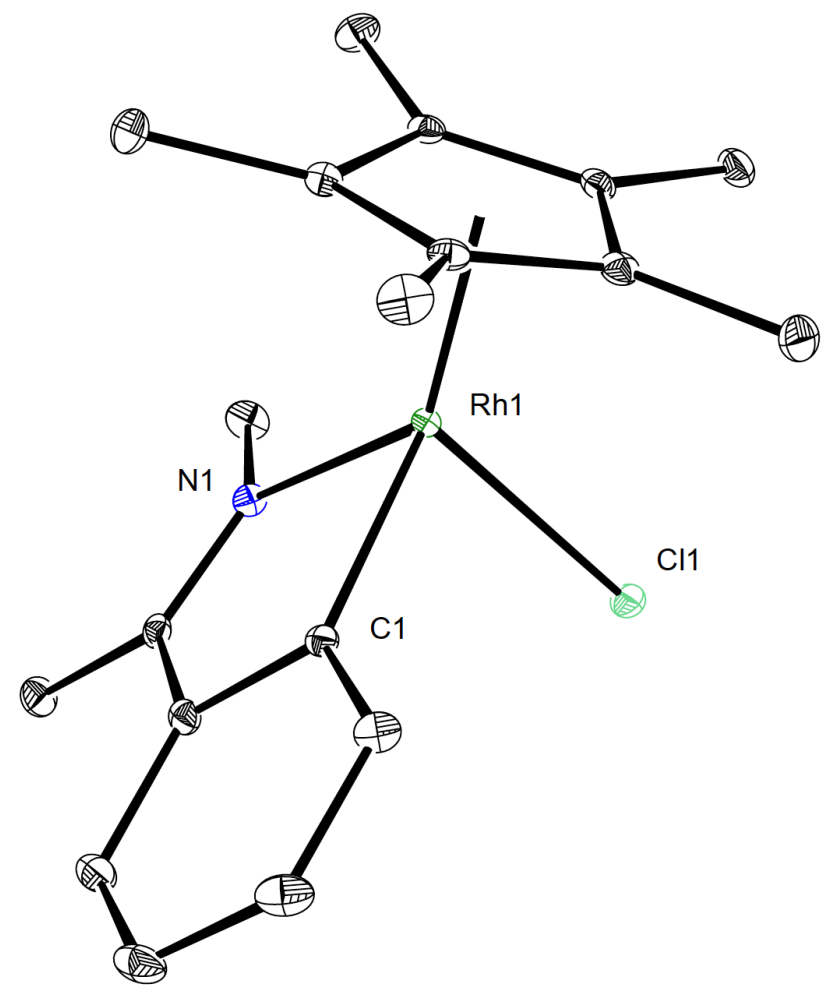

Table S1

Experimental details

\begin{tabular}{|c|c|}
\hline \multicolumn{2}{|l|}{ Crystal data } \\
\hline Chemical formula & $\mathrm{C}_{46} \mathrm{H}_{66} \mathrm{Cl}_{2} \mathrm{~N}_{2} \mathrm{O}_{2} \mathrm{Rh}_{2}$ \\
\hline$M_{\mathrm{r}}$ & 955.72 \\
\hline Crystal system, space group & Monoclinic, $P 2_{1} / n$ \\
\hline Temperature $(\mathrm{K})$ & 100 \\
\hline$a, b, c(\AA)$ & $13.1985(6), 7.9910(4), 21.6147(10)$ \\
\hline$\beta\left(^{\circ}\right)$ & $107.357(1)$ \\
\hline \begin{tabular}{|l|}
$V\left(\AA^{3}\right)$ \\
\end{tabular} & $2175.88(18)$ \\
\hline$Z$ & 2 \\
\hline Radiation type & Mo $K \alpha$ \\
\hline$\mu\left(\mathrm{mm}^{-1}\right)$ & 0.92 \\
\hline Crystal size $(\mathrm{mm})$ & $0.34 \times 0.12 \times 0.11$ \\
\hline
\end{tabular}




\begin{tabular}{|c|c|}
\hline \multicolumn{2}{|l|}{ Data collection } \\
\hline Diffractometer & Bruker D8 Venture Photon 100 CMOS \\
\hline Absorption correction & $\begin{array}{l}\text { Multi-scan } \\
\text { TWINABS BRUKER AXS }\end{array}$ \\
\hline$T_{\min }, T_{\max }$ & $0.641,0.746$ \\
\hline $\begin{array}{l}\text { No. of measured, independent and } \\
\text { observed }[I>2 \sigma(I)] \text { reflections }\end{array}$ & $77631,5621,5004$ \\
\hline$R_{\text {int }}$ & 0.057 \\
\hline$(\sin \theta / \lambda)_{\max }\left(\AA^{-1}\right)$ & 0.676 \\
\hline \multicolumn{2}{|l|}{ Refinement } \\
\hline$R\left[F^{2}>2 \sigma\left(F^{2}\right)\right], w R\left(F^{2}\right), S$ & $0.032,0.084,1.07$ \\
\hline No. of reflections & 5621 \\
\hline No. of parameters & 244 \\
\hline H-atom treatment & H-atom parameters constrained \\
\hline$\left.\Delta\rangle_{\max }, \Delta\right\rangle_{\min }\left(\mathrm{e} \AA^{-3}\right)$ & $0.81,-0.80$ \\
\hline
\end{tabular}

Computer programs: Bruker APEX3, Bruker SAINT, SHELXT 2014/5 (Sheldrick, 2014), SHELXL2018/3 (Sheldrick, 2018).

Document origin: publCIF [Westrip, S. P. (2010). J. Apply. Cryst., 43, 920-925].

full crystallographic data

Computing details

Data collection: Bruker APEX3; cell refinement: Bruker SAINT; data reduction: Bruker SAINT; program(s) used to solve structure: SHELXT 2014/5 (Sheldrick, 2014); program(s) used to refine structure: SHELXL2018/3 (Sheldrick, 2018).

\section{(SMK12)}

Crystal data

\begin{tabular}{|l|l|}
\hline $\mathrm{C}_{46} \mathrm{H}_{66} \mathrm{Cl}_{2} \mathrm{~N}_{2} \mathrm{O}_{2} \mathrm{Rh}_{2}$ & $F(000)=992$ \\
\hline$M_{r}=955.72$ & $D_{\mathrm{x}}=1.459 \mathrm{Mg} \mathrm{m}^{-3}$ \\
\hline Monoclinic, $P 2_{1} / n$ & Mo $K \alpha$ radiation, $\lambda=0.71073 \AA$ \\
\hline$a=13.1985(6) \AA$ & Cell parameters from 9446 reflections \\
\hline$b=7.9910(4) \AA$ & $\theta=2.7-28.7^{\circ}$ \\
\hline
\end{tabular}




\begin{tabular}{|l|l|}
\hline$c=21.6147(10) \AA$ & $\mu=0.92 \mathrm{~mm}^{-1}$ \\
\hline$\beta=107.357(1)^{\circ}$ & $T=100 \mathrm{~K}$ \\
\hline$V=2175.88(18) \AA^{3}$ & Rod, orange \\
\hline$Z=2$ & $0.34 \times 0.12 \times 0.11 \mathrm{~mm}$ \\
\hline
\end{tabular}

\section{Data collection}

\begin{tabular}{|l|l|}
\hline $\begin{array}{l}\text { Bruker D8 Venture Photon 100 CMOS } \\
\text { diffractometer }\end{array}$ & 5004 reflections with $I>2 \sigma(I)$ \\
\hline Radiation source: I $\mu$ S microfocus source & $R_{\text {int }}=0.057$ \\
\hline phi and $\omega$ scans & $\theta_{\max }=28.7^{\circ}, \theta_{\min }=2.1^{\circ}$ \\
\hline $\begin{array}{l}\text { Absorption correction: multi-scan } \\
\text { TWINABS BRUKER AXS }\end{array}$ & $h=-17 \rightarrow 14$ \\
\hline$T_{\min }=0.641, T_{\max }=0.746$ & $k=-10 \rightarrow 10$ \\
\hline 77631 measured reflections & $l=-29 \rightarrow 29$ \\
\hline 5621 independent reflections & \\
\hline
\end{tabular}

\section{Refinement}

\begin{tabular}{|l|l|}
\hline Refinement on $F^{2}$ & 0 restraints \\
\hline Least-squares matrix: full & $\begin{array}{l}\text { Hydrogen site location: inferred from } \\
\text { neighbouring sites }\end{array}$ \\
\hline$R\left[F^{2}>2 \sigma\left(F^{2}\right)\right]=0.032$ & H-atom parameters constrained \\
\hline$w R\left(F^{2}\right)=0.084$ & $\begin{array}{l}w=1 /\left[\sigma^{2}\left(F_{\mathrm{o}}^{2}\right)+(0.0362 P)^{2}+5.3006 P\right] \\
\text { where } P=\left(F_{\mathrm{o}}^{2}+2 F_{\mathrm{c}}^{2}\right) / 3\end{array}$ \\
\hline$S=1.07$ & $(\Delta / \sigma)_{\max }=0.001$ \\
\hline 5621 reflections & $\Delta\rangle_{\max }=0.81 \mathrm{e} \AA^{-3}$ \\
\hline 244 parameters & $\Delta\rangle_{\min }=-0.80 \mathrm{e} \AA^{-3}$ \\
\hline
\end{tabular}

\section{Special details}

Experimental. Collected, solved and refined by Hongyu Zhong

Geometry. All esds (except the esd in the dihedral angle between two 1.s. planes) are estimated using the full covariance matrix. The cell esds are taken into account individually in the estimation of esds in distances, angles and torsion angles; correlations between esds in cell parameters are only used when they are defined by crystal symmetry. An approximate (isotropic) treatment of cell esds is used for estimating esds involving l.s. planes.

Fractional atomic coordinates and isotropic or equivalent isotropic displacement parameters $\left(\AA^{2}\right)$ for (SMK12)

\begin{tabular}{|l|l|l|l|}
\hline$x$ & $y$ & $z$ & $U_{\text {iso }} * / U_{\text {eq }}$ \\
\hline
\end{tabular}




\begin{tabular}{|c|c|c|c|c|}
\hline $\mathrm{C} 1$ & $0.27948(18)$ & $0.6165(3)$ & $0.49107(11)$ & $0.0104(4)$ \\
\hline C1S & $0.2557(3)$ & $-0.1134(5)$ & $0.6519(2)$ & $0.0479(10)$ \\
\hline H1S1 & 0.306726 & -0.184071 & 0.682329 & $0.057^{*}$ \\
\hline H1S2 & 0.241666 & -0.159342 & 0.608644 & $0.057^{*}$ \\
\hline $\mathrm{C} 2$ & $0.2297(2)$ & $0.5181(3)$ & $0.52694(12)$ & $0.0158(5)$ \\
\hline H2 & 0.160073 & 0.483309 & 0.508102 & $0.019^{*}$ \\
\hline $\mathrm{C} 2 \mathrm{~S}$ & $0.2945(3)$ & $0.0627(5)$ & $0.6547(2)$ & $0.0449(9)$ \\
\hline $\mathrm{H} 2 \mathrm{~S} 1$ & 0.369767 & 0.067084 & 0.677685 & $0.054 *$ \\
\hline $\mathrm{H} 2 \mathrm{~S} 2$ & 0.283651 & 0.105292 & 0.611229 & $0.054 *$ \\
\hline C3 & $0.2829(2)$ & $0.4712(4)$ & $0.59061(13)$ & $0.0197(5)$ \\
\hline $\mathrm{H} 3$ & 0.247664 & 0.408999 & 0.614198 & $0.024 *$ \\
\hline O1S & $0.2372(2)$ & $0.1618(3)$ & $0.68750(13)$ & $0.0429(6)$ \\
\hline $\mathrm{C} 4$ & $0.3886(2)$ & $0.5169(4)$ & $0.61902(12)$ & $0.0204(5)$ \\
\hline H4 & 0.423325 & 0.486577 & 0.661620 & $0.024 *$ \\
\hline $\mathrm{C} 4 \mathrm{~S}$ & $0.1776(2)$ & $0.0524(4)$ & $0.71466(15)$ & $0.0294(7)$ \\
\hline H4S1 & 0.112444 & 0.105834 & 0.716339 & $0.035^{*}$ \\
\hline H4S2 & 0.218264 & 0.019898 & 0.758216 & $0.035^{*}$ \\
\hline $\mathrm{C} 5$ & $0.4419(2)$ & $0.6078(3)$ & $0.58367(12)$ & $0.0163(5)$ \\
\hline H5 & 0.513022 & 0.635211 & 0.601930 & $0.020^{*}$ \\
\hline C6 & $0.38764(18)$ & $0.6580(3)$ & $0.52018(11)$ & $0.0121(4)$ \\
\hline $\mathrm{C} 7$ & $0.43749(18)$ & $0.7446(3)$ & $0.47724(11)$ & $0.0115(4)$ \\
\hline $\mathrm{C} 8$ & $0.55356(19)$ & $0.7872(3)$ & $0.49766(13)$ & $0.0171(5)$ \\
\hline H8A & 0.585440 & 0.752770 & 0.541745 & $0.026^{*}$ \\
\hline H8B & 0.587445 & 0.730276 & 0.470070 & $0.026^{*}$ \\
\hline $\mathrm{H} 8 \mathrm{C}$ & 0.562015 & 0.905834 & 0.494215 & $0.026^{*}$ \\
\hline C9 & $0.4125(2)$ & 0.8477 (4) & $0.36931(12)$ & $0.0179(5)$ \\
\hline H9A & 0.354305 & 0.862296 & 0.330572 & $0.027 *$ \\
\hline H9B & 0.444986 & 0.954135 & 0.383345 & $0.027 *$ \\
\hline H9C & 0.463953 & 0.773625 & 0.360636 & $0.027^{*}$ \\
\hline $\mathrm{C} 10$ & $0.06616(18)$ & $0.7849(3)$ & $0.41101(12)$ & $0.0123(4)$ \\
\hline C11 & $0.04676(18)$ & $0.6713(3)$ & $0.35794(12)$ & $0.0130(5)$ \\
\hline C12 & $0.08355(18)$ & 0.7509 (3) & $0.30761(11)$ & $0.0125(4)$ \\
\hline $\mathrm{C} 13$ & $0.12828(17)$ & $0.9058(3)$ & $0.33074(11)$ & $0.0115(4)$ \\
\hline C14 & $0.12450(18)$ & $0.9250(3)$ & $0.39743(11)$ & $0.0116(4)$ \\
\hline C15 & $0.0260(2)$ & $0.7701(4)$ & $0.46884(13)$ & $0.0195(5)$ \\
\hline $\mathrm{H} 15 \mathrm{~A}$ & 0.050851 & 0.863509 & 0.497243 & $0.029 *$ \\
\hline H15B & -0.050157 & 0.769213 & 0.454773 & $0.029 *$ \\
\hline
\end{tabular}




\begin{tabular}{|l|l|l|l|l|}
\hline H15C & 0.051780 & 0.667985 & 0.491511 & $0.029^{*}$ \\
\hline C16 & $-0.0128(2)$ & $0.5094(3)$ & $0.35002(14)$ & $0.0197(5)$ \\
\hline H16A & -0.013652 & 0.458496 & 0.309630 & $0.030^{*}$ \\
\hline H16B & 0.021402 & 0.435593 & 0.385090 & $0.030^{*}$ \\
\hline H16C & -0.084315 & 0.530135 & 0.350263 & $0.030^{*}$ \\
\hline C17 & $0.0741(2)$ & $0.6750(3)$ & $0.24280(12)$ & $0.0169(5)$ \\
\hline H17A & 0.040121 & 0.567809 & 0.239706 & $0.025^{*}$ \\
\hline H17B & 0.032515 & 0.747139 & 0.209249 & $0.025^{*}$ \\
\hline H17C & 0.143545 & 0.661510 & 0.237883 & $0.025^{*}$ \\
\hline C18 & $0.1684(2)$ & $1.0361(3)$ & $0.29424(12)$ & $0.0177(5)$ \\
\hline H18A & 0.194871 & 1.129990 & 0.322106 & $0.027^{*}$ \\
\hline H18B & 0.224474 & 0.989960 & 0.279722 & $0.027^{*}$ \\
\hline H18C & 0.111527 & 1.072194 & 0.257484 & $0.027^{*}$ \\
\hline C19 & $0.1572(2)$ & $1.0784(3)$ & $0.43826(12)$ & $0.0164(5)$ \\
\hline H19A & 0.195234 & 1.152264 & 0.418082 & $0.025^{*}$ \\
\hline H19B & 0.095180 & 1.134332 & 0.442468 & $0.025^{*}$ \\
\hline H19C & 0.202031 & 1.047076 & 0.480413 & $0.025^{*}$ \\
\hline C11 & $0.26563(4)$ & $0.44179(7)$ & $0.36012(3)$ & $0.01368(12)$ \\
\hline N1 & $0.37359(15)$ & $0.7761(3)$ & $0.42008(10)$ & $0.0113(4)$ \\
\hline Rh1 & $0.21661(2)$ & $0.70057(2)$ & $0.39959(2)$ & $0.00824(6)$ \\
\hline C3S & $0.1537(3)$ & $-0.0969(4)$ & $0.6707(2)$ & $0.0410(9)$ \\
\hline H3S1 & 0.092933 & -0.076944 & 0.633052 & $0.049^{*}$ \\
\hline H3S2 & 0.140718 & -0.195829 & 0.693242 & $0.049^{*}$ \\
\hline & & & & \\
\hline
\end{tabular}

\section{Atomic displacement parameters $\left(\AA^{2}\right)$ for (SMK12)}

\begin{tabular}{|l|l|l|l|l|l|l|}
\hline & $U^{11}$ & $U^{22}$ & $U^{33}$ & $U^{12}$ & $U^{13}$ & $U^{23}$ \\
\hline C1 & $0.0123(10)$ & $0.0099(11)$ & $0.0093(10)$ & $0.0021(8)$ & $0.0036(8)$ & $-0.0007(8)$ \\
\hline C1S & $0.045(2)$ & $0.037(2)$ & $0.056(2)$ & $0.0076(17)$ & $0.0059(18)$ & $-0.0165(18)$ \\
\hline C2 & $0.0163(11)$ & $0.0167(12)$ & $0.0166(11)$ & $0.0017(9)$ & $0.0080(9)$ & $0.0025(10)$ \\
\hline C2S & $0.046(2)$ & $0.038(2)$ & $0.061(2)$ & $0.0119(16)$ & $0.0318(19)$ & $0.0146(18)$ \\
\hline C3 & $0.0231(13)$ & $0.0220(14)$ & $0.0181(12)$ & $0.0061(10)$ & $0.0122(10)$ & $0.0068(10)$ \\
\hline O1S & $0.0657(17)$ & $0.0245(12)$ & $0.0500(15)$ & $-0.0094(12)$ & $0.0348(14)$ & $-0.0056(11)$ \\
\hline C4 & $0.0261(13)$ & $0.0248(14)$ & $0.0100(11)$ & $0.0093(11)$ & $0.0049(10)$ & $0.0023(10)$ \\
\hline C4S & $0.0249(14)$ & $0.0362(18)$ & $0.0277(15)$ & $0.0071(13)$ & $0.0090(12)$ & $0.0104(13)$ \\
\hline C5 & $0.0160(11)$ & $0.0190(13)$ & $0.0123(11)$ & $0.0045(10)$ & $0.0016(9)$ & $-0.0014(9)$ \\
\hline C6 & $0.0127(10)$ & $0.0127(11)$ & $0.0106(10)$ & $0.0016(9)$ & $0.0031(8)$ & $-0.0027(9)$ \\
\hline C7 & $0.0099(10)$ & $0.0102(11)$ & $0.0142(11)$ & $0.0001(8)$ & $0.0029(8)$ & $-0.0035(9)$ \\
\hline
\end{tabular}




\begin{tabular}{|l|l|l|l|l|l|l|}
\hline C8 & $0.0103(10)$ & $0.0188(13)$ & $0.0207(12)$ & $-0.0018(9)$ & $0.0023(9)$ & $-0.0013(10)$ \\
\hline C9 & $0.0147(11)$ & $0.0223(13)$ & $0.0179(12)$ & $-0.0021(10)$ & $0.0069(9)$ & $0.0049(10)$ \\
\hline C10 & $0.0088(10)$ & $0.0144(12)$ & $0.0141(11)$ & $0.0033(8)$ & $0.0042(8)$ & $0.0029(9)$ \\
\hline C11 & $0.0080(10)$ & $0.0147(12)$ & $0.0159(11)$ & $0.0018(8)$ & $0.0028(8)$ & $0.0014(9)$ \\
\hline C12 & $0.0089(9)$ & $0.0151(11)$ & $0.0118(11)$ & $0.0018(9)$ & $0.0005(8)$ & $0.0001(9)$ \\
\hline C13 & $0.0109(10)$ & $0.0134(11)$ & $0.0101(10)$ & $0.0044(8)$ & $0.0029(8)$ & $0.0008(9)$ \\
\hline C14 & $0.0106(10)$ & $0.0137(12)$ & $0.0114(10)$ & $0.0015(8)$ & $0.0046(8)$ & $0.0008(9)$ \\
\hline C15 & $0.0182(12)$ & $0.0234(14)$ & $0.0217(13)$ & $0.0018(10)$ & $0.0130(10)$ & $0.0025(11)$ \\
\hline C16 & $0.0136(11)$ & $0.0173(13)$ & $0.0265(13)$ & $-0.0038(10)$ & $0.0036(10)$ & $0.0000(11)$ \\
\hline C17 & $0.0161(11)$ & $0.0196(13)$ & $0.0124(11)$ & $0.0012(9)$ & $0.0003(9)$ & $-0.0024(9)$ \\
\hline C18 & $0.0227(12)$ & $0.0147(12)$ & $0.0180(12)$ & $0.0017(10)$ & $0.0098(10)$ & $0.0035(10)$ \\
\hline C19 & $0.0192(11)$ & $0.0150(12)$ & $0.0164(11)$ & $-0.0002(9)$ & $0.0075(9)$ & $-0.0037(10)$ \\
\hline C11 & $0.0153(2)$ & $0.0117(3)$ & $0.0138(3)$ & $0.0025(2)$ & $0.0039(2)$ & $-0.0014(2)$ \\
\hline N1 & $0.0107(9)$ & $0.0116(10)$ & $0.0126(9)$ & $-0.0005(7)$ & $0.0050(7)$ & $0.0009(8)$ \\
\hline Rh1 & $0.00757(9)$ & $0.00920(10)$ & $0.00829(9)$ & $0.00009(6)$ & $0.00289(6)$ & $0.00023(6)$ \\
\hline C3S & $0.0239(15)$ & $0.0278(17)$ & $0.060(2)$ & $-0.0038(13)$ & $-0.0049(15)$ & $0.0070(16)$ \\
\hline
\end{tabular}

Geometric parameters $\left(\AA,{ }^{\circ}\right)$ for (SMK12)

\begin{tabular}{|l|l|l|l|}
\hline $\mathrm{C} 1-\mathrm{C} 2$ & $1.398(3)$ & $\mathrm{C} 10-\mathrm{C} 14$ & $1.437(3)$ \\
\hline $\mathrm{C} 1-\mathrm{C} 6$ & $1.417(3)$ & $\mathrm{C} 10-\mathrm{C} 15$ & $1.501(3)$ \\
\hline $\mathrm{C} 1-\mathrm{Rh} 1$ & $2.018(2)$ & $\mathrm{C} 10-\mathrm{Rh} 1$ & $2.180(2)$ \\
\hline $\mathrm{C} 1 \mathrm{~S}-\mathrm{C} 2 \mathrm{~S}$ & $1.493(6)$ & $\mathrm{C} 11-\mathrm{C} 12$ & $1.462(3)$ \\
\hline $\mathrm{C} 1 \mathrm{~S}-\mathrm{C} 3 \mathrm{~S}$ & $1.523(5)$ & $\mathrm{C} 11-\mathrm{C} 16$ & $1.497(3)$ \\
\hline $\mathrm{C} 1 \mathrm{~S}-\mathrm{H} 1 \mathrm{~S} 1$ & 0.9700 & $\mathrm{C} 11-\mathrm{Rh} 1$ & $2.165(2)$ \\
\hline $\mathrm{C} 1 \mathrm{~S}-\mathrm{H} 1 \mathrm{~S} 2$ & 0.9700 & $\mathrm{C} 12-\mathrm{C} 13$ & $1.398(4)$ \\
\hline $\mathrm{C} 2-\mathrm{C} 3$ & $1.397(4)$ & $\mathrm{C} 12-\mathrm{C} 17$ & $1.497(3)$ \\
\hline $\mathrm{C} 2-\mathrm{H} 2$ & 0.9300 & $\mathrm{C} 12-\mathrm{Rh} 1$ & $2.262(2)$ \\
\hline $\mathrm{C} 2 \mathrm{~S}-\mathrm{O} 1 \mathrm{~S}$ & $1.421(4)$ & $\mathrm{C} 13-\mathrm{C} 14$ & $1.465(3)$ \\
\hline $\mathrm{C} 2 \mathrm{~S}-\mathrm{H} 2 \mathrm{~S} 1$ & 0.9700 & $\mathrm{C} 13-\mathrm{C} 18$ & $1.496(3)$ \\
\hline $\mathrm{C} 2 \mathrm{~S}-\mathrm{H} 2 \mathrm{~S} 2$ & 0.9700 & $\mathrm{C} 13-\mathrm{Rh} 1$ & $2.286(2)$ \\
\hline $\mathrm{C} 3-\mathrm{C} 4$ & $1.395(4)$ & $\mathrm{C} 14-\mathrm{C} 19$ & $1.497(3)$ \\
\hline $\mathrm{C} 3-\mathrm{H} 3$ & 0.9300 & $\mathrm{C} 14-\mathrm{Rh} 1$ & $2.159(2)$ \\
\hline $\mathrm{O} 1 \mathrm{~S}-\mathrm{C} 4 \mathrm{~S}$ & $1.416(4)$ & $\mathrm{C} 15-\mathrm{H} 15 \mathrm{~A}$ & 0.9600 \\
\hline $\mathrm{C} 4-\mathrm{C} 5$ & $1.388(4)$ & $\mathrm{C} 15-\mathrm{H} 15 \mathrm{~B}$ & 0.9600 \\
\hline $\mathrm{C} 4-\mathrm{H} 4$ & 0.9300 & $\mathrm{C} 15-\mathrm{H} 15 \mathrm{C}$ & 0.9600 \\
\hline $\mathrm{C} 4 \mathrm{~S}-\mathrm{C} 3 \mathrm{~S}$ & $1.499(5)$ & $\mathrm{C} 16-\mathrm{H} 16 \mathrm{~A}$ & 0.9600 \\
\hline $\mathrm{C} 4 \mathrm{~S}-\mathrm{H} 4 \mathrm{~S} 1$ & 0.9700 & $\mathrm{C} 16-\mathrm{H} 16 \mathrm{~B}$ & 0.9600 \\
\hline
\end{tabular}




\begin{tabular}{|c|c|c|c|}
\hline $\mathrm{C} 4 \mathrm{~S}-\mathrm{H} 4 \mathrm{~S} 2$ & 0.9700 & $\mathrm{C} 16-\mathrm{H} 16 \mathrm{C}$ & 0.9600 \\
\hline $\mathrm{C} 5-\mathrm{C} 6$ & $1.404(3)$ & C17-H17A & 0.9600 \\
\hline $\mathrm{C} 5-\mathrm{H} 5$ & 0.9300 & C17-H17B & 0.9600 \\
\hline $\mathrm{C} 6-\mathrm{C} 7$ & $1.462(3)$ & $\mathrm{C} 17-\mathrm{H} 17 \mathrm{C}$ & 0.9600 \\
\hline $\mathrm{C} 7-\mathrm{N} 1$ & $1.296(3)$ & $\mathrm{C} 18-\mathrm{H} 18 \mathrm{~A}$ & 0.9600 \\
\hline $\mathrm{C} 7-\mathrm{C} 8$ & $1.501(3)$ & $\mathrm{C} 18-\mathrm{H} 18 \mathrm{~B}$ & 0.9600 \\
\hline $\mathrm{C} 8-\mathrm{H} 8 \mathrm{~A}$ & 0.9600 & $\mathrm{C} 18-\mathrm{H} 18 \mathrm{C}$ & 0.9600 \\
\hline $\mathrm{C} 8-\mathrm{H} 8 \mathrm{~B}$ & 0.9600 & C19-H19A & 0.9600 \\
\hline $\mathrm{C} 8-\mathrm{H} 8 \mathrm{C}$ & 0.9600 & $\mathrm{C} 19-\mathrm{H} 19 \mathrm{~B}$ & 0.9600 \\
\hline $\mathrm{C} 9-\mathrm{N} 1$ & $1.460(3)$ & $\mathrm{C} 19-\mathrm{H} 19 \mathrm{C}$ & 0.9600 \\
\hline $\mathrm{C} 9-\mathrm{H} 9 \mathrm{~A}$ & 0.9600 & $\mathrm{Cl1}-\mathrm{Rh} 1$ & $2.3983(6)$ \\
\hline C9-H9B & 0.9600 & $\mathrm{~N} 1-\mathrm{Rh} 1$ & 2.0751 (19) \\
\hline $\mathrm{C} 9-\mathrm{H} 9 \mathrm{C}$ & 0.9600 & $\mathrm{C} 3 \mathrm{~S}-\mathrm{H} 3 \mathrm{~S} 1$ & 0.9700 \\
\hline $\mathrm{C} 10-\mathrm{C} 11$ & $1.425(3)$ & $\mathrm{C} 3 \mathrm{~S}-\mathrm{H} 3 \mathrm{~S} 2$ & 0.9700 \\
\hline $\mathrm{C} 2-\mathrm{C} 1-\mathrm{C} 6$ & $117.6(2)$ & $\mathrm{C} 18-\mathrm{C} 13-\mathrm{Rh} 1$ & $130.61(16)$ \\
\hline $\mathrm{C} 2-\mathrm{C} 1-\mathrm{Rh} 1$ & $127.37(17)$ & $\mathrm{C} 10-\mathrm{C} 14-\mathrm{C} 13$ & $107.3(2)$ \\
\hline $\mathrm{C} 6-\mathrm{C} 1-\mathrm{Rh} 1$ & $115.00(17)$ & $\mathrm{C} 10-\mathrm{C} 14-\mathrm{C} 19$ & $126.5(2)$ \\
\hline $\mathrm{C} 2 \mathrm{~S}-\mathrm{C} 1 \mathrm{~S}-\mathrm{C} 3 \mathrm{~S}$ & $103.1(3)$ & $\mathrm{C} 13-\mathrm{C} 14-\mathrm{C} 19$ & $125.3(2)$ \\
\hline $\mathrm{C} 2 \mathrm{~S}-\mathrm{C} 1 \mathrm{~S}-\mathrm{H} 1 \mathrm{~S} 1$ & 111.1 & $\mathrm{C} 10-\mathrm{C} 14-\mathrm{Rh} 1$ & $71.43(13)$ \\
\hline $\mathrm{C} 3 \mathrm{~S}-\mathrm{C} 1 \mathrm{~S}-\mathrm{H} 1 \mathrm{~S} 1$ & 111.1 & $\mathrm{C} 13-\mathrm{C} 14-\mathrm{Rh} 1$ & $75.53(13)$ \\
\hline $\mathrm{C} 2 \mathrm{~S}-\mathrm{C} 1 \mathrm{~S}-\mathrm{H} 1 \mathrm{~S} 2$ & 111.1 & $\mathrm{C} 19-\mathrm{C} 14-\mathrm{Rh} 1$ & $127.32(16)$ \\
\hline $\mathrm{C} 3 \mathrm{~S}-\mathrm{C} 1 \mathrm{~S}-\mathrm{H} 1 \mathrm{~S} 2$ & 111.1 & $\mathrm{C} 10-\mathrm{C} 15-\mathrm{H} 15 \mathrm{~A}$ & 109.5 \\
\hline $\mathrm{H} 1 \mathrm{~S} 1-\mathrm{C} 1 \mathrm{~S}-\mathrm{H} 1 \mathrm{~S} 2$ & 109.1 & $\mathrm{C} 10-\mathrm{C} 15-\mathrm{H} 15 \mathrm{~B}$ & 109.5 \\
\hline $\mathrm{C} 3-\mathrm{C} 2-\mathrm{C} 1$ & $121.1(2)$ & $\mathrm{H} 15 \mathrm{~A}-\mathrm{C} 15-\mathrm{H} 15 \mathrm{~B}$ & 109.5 \\
\hline $\mathrm{C} 3-\mathrm{C} 2-\mathrm{H} 2$ & 119.5 & $\mathrm{C} 10-\mathrm{C} 15-\mathrm{H} 15 \mathrm{C}$ & 109.5 \\
\hline $\mathrm{C} 1-\mathrm{C} 2-\mathrm{H} 2$ & 119.5 & $\mathrm{H} 15 \mathrm{~A}-\mathrm{C} 15-\mathrm{H} 15 \mathrm{C}$ & 109.5 \\
\hline $\mathrm{O} 1 \mathrm{~S}-\mathrm{C} 2 \mathrm{~S}-\mathrm{C} 1 \mathrm{~S}$ & $108.6(3)$ & $\mathrm{H} 15 \mathrm{~B}-\mathrm{C} 15-\mathrm{H} 15 \mathrm{C}$ & 109.5 \\
\hline $\mathrm{O} 1 \mathrm{~S}-\mathrm{C} 2 \mathrm{~S}-\mathrm{H} 2 \mathrm{~S} 1$ & 110.0 & $\mathrm{C} 11-\mathrm{C} 16-\mathrm{H} 16 \mathrm{~A}$ & 109.5 \\
\hline $\mathrm{C} 1 \mathrm{~S}-\mathrm{C} 2 \mathrm{~S}-\mathrm{H} 2 \mathrm{~S} 1$ & 110.0 & $\mathrm{C} 11-\mathrm{C} 16-\mathrm{H} 16 \mathrm{~B}$ & 109.5 \\
\hline $\mathrm{O} 1 \mathrm{~S}-\mathrm{C} 2 \mathrm{~S}-\mathrm{H} 2 \mathrm{~S} 2$ & 110.0 & $\mathrm{H} 16 \mathrm{~A}-\mathrm{C} 16-\mathrm{H} 16 \mathrm{~B}$ & 109.5 \\
\hline $\mathrm{C} 1 \mathrm{~S}-\mathrm{C} 2 \mathrm{~S}-\mathrm{H} 2 \mathrm{~S} 2$ & 110.0 & $\mathrm{C} 11-\mathrm{C} 16-\mathrm{H} 16 \mathrm{C}$ & 109.5 \\
\hline $\mathrm{H} 2 \mathrm{~S} 1-\mathrm{C} 2 \mathrm{~S}-\mathrm{H} 2 \mathrm{~S} 2$ & 108.4 & $\mathrm{H} 16 \mathrm{~A}-\mathrm{C} 16-\mathrm{H} 16 \mathrm{C}$ & 109.5 \\
\hline $\mathrm{C} 4-\mathrm{C} 3-\mathrm{C} 2$ & $120.4(2)$ & $\mathrm{H} 16 \mathrm{~B}-\mathrm{C} 16-\mathrm{H} 16 \mathrm{C}$ & 109.5 \\
\hline $\mathrm{C} 4-\mathrm{C} 3-\mathrm{H} 3$ & 119.8 & $\mathrm{C} 12-\mathrm{C} 17-\mathrm{H} 17 \mathrm{~A}$ & 109.5 \\
\hline $\mathrm{C} 2-\mathrm{C} 3-\mathrm{H} 3$ & 119.8 & $\mathrm{C} 12-\mathrm{C} 17-\mathrm{H} 17 \mathrm{~B}$ & 109.5 \\
\hline $\mathrm{C} 4 \mathrm{~S}-\mathrm{O} 1 \mathrm{~S}-\mathrm{C} 2 \mathrm{~S}$ & $107.9(3)$ & $\mathrm{H} 17 \mathrm{~A}-\mathrm{C} 17-\mathrm{H} 17 \mathrm{~B}$ & 109.5 \\
\hline
\end{tabular}




\begin{tabular}{|c|c|c|c|}
\hline $\mathrm{C} 5-\mathrm{C} 4-\mathrm{C} 3$ & $119.9(2)$ & $\mathrm{C} 12-\mathrm{C} 17-\mathrm{H} 17 \mathrm{C}$ & 109.5 \\
\hline $\mathrm{C} 5-\mathrm{C} 4-\mathrm{H} 4$ & 120.0 & $\mathrm{H} 17 \mathrm{~A}-\mathrm{C} 17-\mathrm{H} 17 \mathrm{C}$ & 109.5 \\
\hline $\mathrm{C} 3-\mathrm{C} 4-\mathrm{H} 4$ & 120.0 & $\mathrm{H} 17 \mathrm{~B}-\mathrm{C} 17-\mathrm{H} 17 \mathrm{C}$ & 109.5 \\
\hline $\mathrm{O} 1 \mathrm{~S}-\mathrm{C} 4 \mathrm{~S}-\mathrm{C} 3 \mathrm{~S}$ & $105.5(3)$ & $\mathrm{C} 13-\mathrm{C} 18-\mathrm{H} 18 \mathrm{~A}$ & 109.5 \\
\hline $\mathrm{O} 1 \mathrm{~S}-\mathrm{C} 4 \mathrm{~S}-\mathrm{H} 4 \mathrm{~S} 1$ & 110.6 & $\mathrm{C} 13-\mathrm{C} 18-\mathrm{H} 18 \mathrm{~B}$ & 109.5 \\
\hline $\mathrm{C} 3 \mathrm{~S}-\mathrm{C} 4 \mathrm{~S}-\mathrm{H} 4 \mathrm{~S} 1$ & 110.6 & $\mathrm{H} 18 \mathrm{~A}-\mathrm{C} 18-\mathrm{H} 18 \mathrm{~B}$ & 109.5 \\
\hline $\mathrm{O} 1 \mathrm{~S}-\mathrm{C} 4 \mathrm{~S}-\mathrm{H} 4 \mathrm{~S} 2$ & 110.6 & $\mathrm{C} 13-\mathrm{C} 18-\mathrm{H} 18 \mathrm{C}$ & 109.5 \\
\hline $\mathrm{C} 3 \mathrm{~S}-\mathrm{C} 4 \mathrm{~S}-\mathrm{H} 4 \mathrm{~S} 2$ & 110.6 & $\mathrm{H} 18 \mathrm{~A}-\mathrm{C} 18-\mathrm{H} 18 \mathrm{C}$ & 109.5 \\
\hline $\mathrm{H} 4 \mathrm{~S} 1-\mathrm{C} 4 \mathrm{~S}-\mathrm{H} 4 \mathrm{~S} 2$ & 108.8 & $\mathrm{H} 18 \mathrm{~B}-\mathrm{C} 18-\mathrm{H} 18 \mathrm{C}$ & 109.5 \\
\hline $\mathrm{C} 4-\mathrm{C} 5-\mathrm{C} 6$ & $119.6(2)$ & $\mathrm{C} 14-\mathrm{C} 19-\mathrm{H} 19 \mathrm{~A}$ & 109.5 \\
\hline $\mathrm{C} 4-\mathrm{C} 5-\mathrm{H} 5$ & 120.2 & $\mathrm{C} 14-\mathrm{C} 19-\mathrm{H} 19 \mathrm{~B}$ & 109.5 \\
\hline $\mathrm{C} 6-\mathrm{C} 5-\mathrm{H} 5$ & 120.2 & $\mathrm{H} 19 \mathrm{~A}-\mathrm{C} 19-\mathrm{H} 19 \mathrm{~B}$ & 109.5 \\
\hline $\mathrm{C} 5-\mathrm{C} 6-\mathrm{C} 1$ & $121.3(2)$ & $\mathrm{C} 14-\mathrm{C} 19-\mathrm{H} 19 \mathrm{C}$ & 109.5 \\
\hline $\mathrm{C} 5-\mathrm{C} 6-\mathrm{C} 7$ & $124.1(2)$ & $\mathrm{H} 19 \mathrm{~A}-\mathrm{C} 19-\mathrm{H} 19 \mathrm{C}$ & 109.5 \\
\hline $\mathrm{C} 1-\mathrm{C} 6-\mathrm{C} 7$ & $114.4(2)$ & $\mathrm{H} 19 \mathrm{~B}-\mathrm{C} 19-\mathrm{H} 19 \mathrm{C}$ & 109.5 \\
\hline $\mathrm{N} 1-\mathrm{C} 7-\mathrm{C} 6$ & $114.2(2)$ & $\mathrm{C} 7-\mathrm{N} 1-\mathrm{C} 9$ & $121.2(2)$ \\
\hline $\mathrm{N} 1-\mathrm{C} 7-\mathrm{C} 8$ & $123.6(2)$ & $\mathrm{C} 7-\mathrm{N} 1-\mathrm{Rh} 1$ & $118.12(16)$ \\
\hline $\mathrm{C} 6-\mathrm{C} 7-\mathrm{C} 8$ & $122.3(2)$ & C9-N1-Rh1 & $120.43(15)$ \\
\hline $\mathrm{C} 7-\mathrm{C} 8-\mathrm{H} 8 \mathrm{~A}$ & 109.5 & $\mathrm{C} 1-\mathrm{Rh} 1-\mathrm{N} 1$ & $78.18(9)$ \\
\hline $\mathrm{C} 7-\mathrm{C} 8-\mathrm{H} 8 \mathrm{~B}$ & 109.5 & $\mathrm{C} 1-\mathrm{Rh} 1-\mathrm{C} 14$ & $111.22(9)$ \\
\hline $\mathrm{H} 8 \mathrm{~A}-\mathrm{C} 8-\mathrm{H} 8 \mathrm{~B}$ & 109.5 & $\mathrm{~N} 1-\mathrm{Rh} 1-\mathrm{C} 14$ & $106.43(8)$ \\
\hline $\mathrm{C} 7-\mathrm{C} 8-\mathrm{H} 8 \mathrm{C}$ & 109.5 & $\mathrm{C} 1-\mathrm{Rh} 1-\mathrm{C} 11$ & $116.34(9)$ \\
\hline $\mathrm{H} 8 \mathrm{~A}-\mathrm{C} 8-\mathrm{H} 8 \mathrm{C}$ & 109.5 & $\mathrm{~N} 1-\mathrm{Rh} 1-\mathrm{C} 11$ & $164.65(9)$ \\
\hline $\mathrm{H} 8 \mathrm{~B}-\mathrm{C} 8-\mathrm{H} 8 \mathrm{C}$ & 109.5 & $\mathrm{C} 14-\mathrm{Rh} 1-\mathrm{C} 11$ & $64.79(9)$ \\
\hline $\mathrm{N} 1-\mathrm{C} 9-\mathrm{H} 9 \mathrm{~A}$ & 109.5 & $\mathrm{C} 1-\mathrm{Rh} 1-\mathrm{C} 10$ & $96.56(9)$ \\
\hline $\mathrm{N} 1-\mathrm{C} 9-\mathrm{H} 9 \mathrm{~B}$ & 109.5 & $\mathrm{~N} 1-\mathrm{Rh} 1-\mathrm{C} 10$ & $140.46(9)$ \\
\hline $\mathrm{H} 9 \mathrm{~A}-\mathrm{C} 9-\mathrm{H} 9 \mathrm{~B}$ & 109.5 & $\mathrm{C} 14-\mathrm{Rh} 1-\mathrm{C} 10$ & $38.69(9)$ \\
\hline $\mathrm{N} 1-\mathrm{C} 9-\mathrm{H} 9 \mathrm{C}$ & 109.5 & $\mathrm{C} 11-\mathrm{Rh} 1-\mathrm{C} 10$ & $38.29(9)$ \\
\hline $\mathrm{H} 9 \mathrm{~A}-\mathrm{C} 9-\mathrm{H} 9 \mathrm{C}$ & 109.5 & $\mathrm{C} 1-\mathrm{Rh} 1-\mathrm{C} 12$ & $154.84(9)$ \\
\hline $\mathrm{H} 9 \mathrm{~B}-\mathrm{C} 9-\mathrm{H} 9 \mathrm{C}$ & 109.5 & $\mathrm{~N} 1-\mathrm{Rh} 1-\mathrm{C} 12$ & $126.87(8)$ \\
\hline $\mathrm{C} 11-\mathrm{C} 10-\mathrm{C} 14$ & $108.1(2)$ & $\mathrm{C} 14-\mathrm{Rh} 1-\mathrm{C} 12$ & $63.18(9)$ \\
\hline $\mathrm{C} 11-\mathrm{C} 10-\mathrm{C} 15$ & $126.6(2)$ & $\mathrm{C} 11-\mathrm{Rh} 1-\mathrm{C} 12$ & $38.50(9)$ \\
\hline $\mathrm{C} 14-\mathrm{C} 10-\mathrm{C} 15$ & $125.1(2)$ & $\mathrm{C} 10-\mathrm{Rh} 1-\mathrm{C} 12$ & $63.25(9)$ \\
\hline $\mathrm{C} 11-\mathrm{C} 10-\mathrm{Rh} 1$ & $70.29(13)$ & $\mathrm{C} 1-\mathrm{Rh} 1-\mathrm{C} 13$ & $149.04(9)$ \\
\hline $\mathrm{C} 14-\mathrm{C} 10-\mathrm{Rh} 1$ & $69.88(13)$ & N1-Rh1-C13 & $102.30(8)$ \\
\hline $\mathrm{C} 15-\mathrm{C} 10-\mathrm{Rh} 1$ & $129.02(17)$ & $\mathrm{C} 14-\mathrm{Rh} 1-\mathrm{C} 13$ & $38.36(8)$ \\
\hline $\mathrm{C} 10-\mathrm{C} 11-\mathrm{C} 12$ & $107.6(2)$ & C11-Rh1-C13 & $62.81(9)$ \\
\hline
\end{tabular}




\begin{tabular}{|c|c|c|c|}
\hline $\mathrm{C} 10-\mathrm{C} 11-\mathrm{C} 16$ & $127.2(2)$ & $\mathrm{C} 10-\mathrm{Rh} 1-\mathrm{C} 13$ & $63.07(8)$ \\
\hline $\mathrm{C} 12-\mathrm{C} 11-\mathrm{C} 16$ & $124.8(2)$ & $\mathrm{C} 12-\mathrm{Rh} 1-\mathrm{C} 13$ & $35.80(9)$ \\
\hline $\mathrm{C} 10-\mathrm{C} 11-\mathrm{Rh} 1$ & $71.42(13)$ & $\mathrm{C} 1-\mathrm{Rh} 1-\mathrm{Cl} 1$ & $89.18(7)$ \\
\hline $\mathrm{C} 12-\mathrm{C} 11-\mathrm{Rh} 1$ & $74.33(13)$ & $\mathrm{N} 1-\mathrm{Rh} 1-\mathrm{Cl1}$ & $88.21(6)$ \\
\hline $\mathrm{C} 16-\mathrm{C} 11-\mathrm{Rh} 1$ & $125.73(17)$ & $\mathrm{C} 14-\mathrm{Rh} 1-\mathrm{Cl} 1$ & $156.68(6)$ \\
\hline $\mathrm{C} 13-\mathrm{C} 12-\mathrm{C} 11$ & $108.5(2)$ & $\mathrm{C} 11-\mathrm{Rh} 1-\mathrm{C} 11$ & $96.59(7)$ \\
\hline $\mathrm{C} 13-\mathrm{C} 12-\mathrm{C} 17$ & $127.0(2)$ & $\mathrm{C} 10-\mathrm{Rh} 1-\mathrm{Cl} 1$ & $131.19(7)$ \\
\hline $\mathrm{C} 11-\mathrm{C} 12-\mathrm{C} 17$ & $124.5(2)$ & $\mathrm{C} 12-\mathrm{Rh} 1-\mathrm{Cl1}$ & $93.54(7)$ \\
\hline $\mathrm{C} 13-\mathrm{C} 12-\mathrm{Rh} 1$ & $73.06(13)$ & $\mathrm{C} 13-\mathrm{Rh} 1-\mathrm{C} 11$ & $121.74(6)$ \\
\hline $\mathrm{C} 11-\mathrm{C} 12-\mathrm{Rh} 1$ & $67.17(13)$ & $\mathrm{C} 4 \mathrm{~S}-\mathrm{C} 3 \mathrm{~S}-\mathrm{C} 1 \mathrm{~S}$ & $101.7(3)$ \\
\hline $\mathrm{C} 17-\mathrm{C} 12-\mathrm{Rh} 1$ & $125.23(17)$ & $\mathrm{C} 4 \mathrm{~S}-\mathrm{C} 3 \mathrm{~S}-\mathrm{H} 3 \mathrm{~S} 1$ & 111.4 \\
\hline $\mathrm{C} 12-\mathrm{C} 13-\mathrm{C} 14$ & $108.0(2)$ & $\mathrm{C} 1 \mathrm{~S}-\mathrm{C} 3 \mathrm{~S}-\mathrm{H} 3 \mathrm{~S} 1$ & 111.4 \\
\hline $\mathrm{C} 12-\mathrm{C} 13-\mathrm{C} 18$ & $127.3(2)$ & $\mathrm{C} 4 \mathrm{~S}-\mathrm{C} 3 \mathrm{~S}-\mathrm{H} 3 \mathrm{~S} 2$ & 111.4 \\
\hline $\mathrm{C} 14-\mathrm{C} 13-\mathrm{C} 18$ & $124.5(2)$ & $\mathrm{C} 1 \mathrm{~S}-\mathrm{C} 3 \mathrm{~S}-\mathrm{H} 3 \mathrm{~S} 2$ & 111.4 \\
\hline $\mathrm{C} 12-\mathrm{C} 13-\mathrm{Rh} 1$ & $71.14(14)$ & $\mathrm{H} 3 \mathrm{~S} 1-\mathrm{C} 3 \mathrm{~S}-\mathrm{H} 3 \mathrm{~S} 2$ & 109.3 \\
\hline $\mathrm{C} 14-\mathrm{C} 13-\mathrm{Rh} 1$ & $66.12(13)$ & & \\
\hline $\mathrm{C} 6-\mathrm{C} 1-\mathrm{C} 2-\mathrm{C} 3$ & $3.8(4)$ & $\begin{array}{l}\mathrm{C} 10-\mathrm{C} 11-\mathrm{C} 12- \\
\mathrm{Rh} 1\end{array}$ & $64.15(15)$ \\
\hline $\mathrm{Rh} 1-\mathrm{C} 1-\mathrm{C} 2-\mathrm{C} 3$ & $-178.96(19)$ & $\begin{array}{l}\mathrm{C} 16-\mathrm{C} 11-\mathrm{C} 12- \\
\mathrm{Rh} 1\end{array}$ & $-122.9(2)$ \\
\hline $\begin{array}{l}\mathrm{C} 3 \mathrm{~S}-\mathrm{C} 1 \mathrm{~S}-\mathrm{C} 2 \mathrm{~S}- \\
\mathrm{O} 1 \mathrm{~S}\end{array}$ & $13.6(4)$ & $\begin{array}{l}\mathrm{C} 11-\mathrm{C} 12-\mathrm{C} 13- \\
\mathrm{C} 14\end{array}$ & $2.2(3)$ \\
\hline $\mathrm{C} 1-\mathrm{C} 2-\mathrm{C} 3-\mathrm{C} 4$ & $-2.3(4)$ & $\begin{array}{l}\mathrm{C} 17-\mathrm{C} 12-\mathrm{C} 13- \\
\mathrm{C} 14\end{array}$ & $-177.6(2)$ \\
\hline $\begin{array}{l}\mathrm{C} 1 \mathrm{~S}-\mathrm{C} 2 \mathrm{~S}-\mathrm{O} 1 \mathrm{~S}- \\
\mathrm{C} 4 \mathrm{~S}\end{array}$ & $9.6(4)$ & $\begin{array}{l}\mathrm{Rh} 1-\mathrm{C} 12-\mathrm{C} 13- \\
\mathrm{C} 14\end{array}$ & $-55.86(15)$ \\
\hline $\mathrm{C} 2-\mathrm{C} 3-\mathrm{C} 4-\mathrm{C} 5$ & $-0.8(4)$ & $\begin{array}{l}\mathrm{C} 11-\mathrm{C} 12-\mathrm{C} 13- \\
\mathrm{C} 18\end{array}$ & $-174.8(2)$ \\
\hline $\begin{array}{l}\mathrm{C} 2 \mathrm{~S}-\mathrm{O} 1 \mathrm{~S}-\mathrm{C} 4 \mathrm{~S}- \\
\mathrm{C} 3 \mathrm{~S}\end{array}$ & $-29.5(4)$ & $\begin{array}{l}\mathrm{C} 17-\mathrm{C} 12-\mathrm{C} 13- \\
\mathrm{C} 18\end{array}$ & $5.5(4)$ \\
\hline $\mathrm{C} 3-\mathrm{C} 4-\mathrm{C} 5-\mathrm{C} 6$ & $2.2(4)$ & $\begin{array}{l}\text { Rh1-C12-C13- } \\
\text { C18 }\end{array}$ & $127.2(2)$ \\
\hline $\mathrm{C} 4-\mathrm{C} 5-\mathrm{C} 6-\mathrm{C} 1$ & $-0.6(4)$ & $\begin{array}{l}\mathrm{C} 11-\mathrm{C} 12-\mathrm{C} 13- \\
\mathrm{Rh} 1\end{array}$ & $58.02(16)$ \\
\hline $\mathrm{C} 4-\mathrm{C} 5-\mathrm{C} 6-\mathrm{C} 7$ & $-175.9(2)$ & $\begin{array}{l}\mathrm{C} 17-\mathrm{C} 12-\mathrm{C} 13- \\
\mathrm{Rh} 1\end{array}$ & $-121.7(2)$ \\
\hline $\mathrm{C} 2-\mathrm{C} 1-\mathrm{C} 6-\mathrm{C} 5$ & $-2.4(4)$ & $\begin{array}{l}\mathrm{C} 11-\mathrm{C} 10-\mathrm{C} 14- \\
\mathrm{C} 13\end{array}$ & $7.5(3)$ \\
\hline $\mathrm{Rh} 1-\mathrm{C} 1-\mathrm{C} 6-\mathrm{C} 5$ & $-179.99(19)$ & $\begin{array}{l}\mathrm{C} 15-\mathrm{C} 10-\mathrm{C} 14- \\
\mathrm{C} 13\end{array}$ & $-168.2(2)$ \\
\hline
\end{tabular}




\begin{tabular}{|c|c|c|c|}
\hline $\mathrm{C} 2-\mathrm{C} 1-\mathrm{C} 6-\mathrm{C} 7$ & $173.4(2)$ & $\begin{array}{l}\text { Rh1-C10-C14- } \\
\text { C13 }\end{array}$ & $67.63(15)$ \\
\hline $\mathrm{Rh} 1-\mathrm{C} 1-\mathrm{C} 6-\mathrm{C} 7$ & $-4.2(3)$ & $\begin{array}{l}\mathrm{C} 11-\mathrm{C} 10-\mathrm{C} 14- \\
\mathrm{C} 19\end{array}$ & $176.7(2)$ \\
\hline $\mathrm{C} 5-\mathrm{C} 6-\mathrm{C} 7-\mathrm{N} 1$ & $178.5(2)$ & $\begin{array}{l}\mathrm{C} 15-\mathrm{C} 10-\mathrm{C} 14- \\
\mathrm{C} 19\end{array}$ & $1.0(4)$ \\
\hline $\mathrm{C} 1-\mathrm{C} 6-\mathrm{C} 7-\mathrm{N} 1$ & $2.8(3)$ & $\begin{array}{l}\text { Rh1-C10-C14- } \\
\text { C19 }\end{array}$ & $-123.2(2)$ \\
\hline $\mathrm{C} 5-\mathrm{C} 6-\mathrm{C} 7-\mathrm{C} 8$ & $0.4(4)$ & $\begin{array}{l}\mathrm{C} 11-\mathrm{C} 10-\mathrm{C} 14- \\
\mathrm{Rh} 1\end{array}$ & $-60.18(16)$ \\
\hline $\mathrm{C} 1-\mathrm{C} 6-\mathrm{C} 7-\mathrm{C} 8$ & $-175.3(2)$ & $\begin{array}{l}\mathrm{C} 15-\mathrm{C} 10-\mathrm{C} 14- \\
\mathrm{Rh} 1\end{array}$ & $124.2(2)$ \\
\hline $\begin{array}{l}\mathrm{C} 14-\mathrm{C} 10-\mathrm{C} 11- \\
\mathrm{C} 12\end{array}$ & $-6.2(3)$ & $\begin{array}{l}\mathrm{C} 12-\mathrm{C} 13-\mathrm{C} 14- \\
\mathrm{C} 10\end{array}$ & $-5.9(3)$ \\
\hline $\begin{array}{l}\mathrm{C} 15-\mathrm{C} 10-\mathrm{C} 11- \\
\mathrm{C} 12\end{array}$ & $169.4(2)$ & $\begin{array}{l}\mathrm{C} 18-\mathrm{C} 13-\mathrm{C} 14- \\
\mathrm{C} 10\end{array}$ & $171.1(2)$ \\
\hline $\begin{array}{l}\mathrm{Rh} 1-\mathrm{C} 10-\mathrm{C} 11- \\
\mathrm{C} 12\end{array}$ & $-66.08(15)$ & $\begin{array}{l}\text { Rh1-C13-C14- } \\
\text { C10 }\end{array}$ & $-64.87(15)$ \\
\hline $\begin{array}{l}\mathrm{C} 14-\mathrm{C} 10-\mathrm{C} 11- \\
\mathrm{C} 16\end{array}$ & $-178.9(2)$ & $\begin{array}{l}\mathrm{C} 12-\mathrm{C} 13-\mathrm{C} 14- \\
\mathrm{C} 19\end{array}$ & $-175.3(2)$ \\
\hline $\begin{array}{l}\mathrm{C} 15-\mathrm{C} 10-\mathrm{C} 11- \\
\mathrm{C} 16\end{array}$ & $-3.3(4)$ & $\begin{array}{l}\mathrm{C} 18-\mathrm{C} 13-\mathrm{C} 14- \\
\mathrm{C} 19\end{array}$ & $1.7(4)$ \\
\hline $\begin{array}{l}\mathrm{Rh} 1-\mathrm{C} 10-\mathrm{C} 11- \\
\mathrm{C} 16\end{array}$ & $121.2(2)$ & $\begin{array}{l}\text { Rh1-C13-C14- } \\
\text { C19 }\end{array}$ & $125.8(2)$ \\
\hline $\begin{array}{l}\mathrm{C} 14-\mathrm{C} 10-\mathrm{C} 11- \\
\mathrm{Rh} 1\end{array}$ & $59.92(16)$ & $\begin{array}{l}\mathrm{C} 12-\mathrm{C} 13-\mathrm{C} 14- \\
\mathrm{Rh} 1\end{array}$ & $58.93(16)$ \\
\hline $\begin{array}{l}\mathrm{C} 15-\mathrm{C} 10-\mathrm{C} 11- \\
\mathrm{Rh} 1\end{array}$ & $-124.5(2)$ & $\begin{array}{l}\text { C18-C13-C14- } \\
\text { Rh1 }\end{array}$ & $-124.0(2)$ \\
\hline $\begin{array}{l}\mathrm{C} 10-\mathrm{C} 11-\mathrm{C} 12- \\
\mathrm{C} 13\end{array}$ & $2.5(3)$ & $\mathrm{C} 6-\mathrm{C} 7-\mathrm{N} 1-\mathrm{C} 9$ & $-174.2(2)$ \\
\hline $\begin{array}{l}\mathrm{C} 16-\mathrm{C} 11-\mathrm{C} 12- \\
\mathrm{C} 13\end{array}$ & $175.4(2)$ & $\mathrm{C} 8-\mathrm{C} 7-\mathrm{N} 1-\mathrm{C} 9$ & $3.9(4)$ \\
\hline $\begin{array}{l}\mathrm{Rh} 1-\mathrm{C} 11-\mathrm{C} 12- \\
\mathrm{C} 13\end{array}$ & $-61.69(16)$ & $\mathrm{C} 6-\mathrm{C} 7-\mathrm{N} 1-\mathrm{Rh} 1$ & $-0.2(3)$ \\
\hline $\begin{array}{l}\mathrm{C} 10-\mathrm{C} 11-\mathrm{C} 12- \\
\mathrm{C} 17\end{array}$ & $-177.8(2)$ & $\mathrm{C} 8-\mathrm{C} 7-\mathrm{N} 1-\mathrm{Rh} 1$ & $177.91(18)$ \\
\hline $\begin{array}{l}\mathrm{C} 16-\mathrm{C} 11-\mathrm{C} 12- \\
\mathrm{C} 17\end{array}$ & $-4.9(4)$ & $\begin{array}{l}\mathrm{O} 1 \mathrm{~S}-\mathrm{C} 4 \mathrm{~S}-\mathrm{C} 3 \mathrm{~S}- \\
\mathrm{C} 1 \mathrm{~S}\end{array}$ & $36.8(3)$ \\
\hline $\begin{array}{l}\mathrm{Rh} 1-\mathrm{C} 11-\mathrm{C} 12- \\
\mathrm{C} 17\end{array}$ & $118.0(2)$ & $\begin{array}{l}\mathrm{C} 2 \mathrm{~S}-\mathrm{C} 1 \mathrm{~S}-\mathrm{C} 3 \mathrm{~S}- \\
\mathrm{C} 4 \mathrm{~S}\end{array}$ & $-29.9(4)$ \\
\hline
\end{tabular}

Document origin: publCIF [Westrip, S. P. (2010). J. Apply. Cryst., 43, 920-925]. 


\section{Compound 2:}

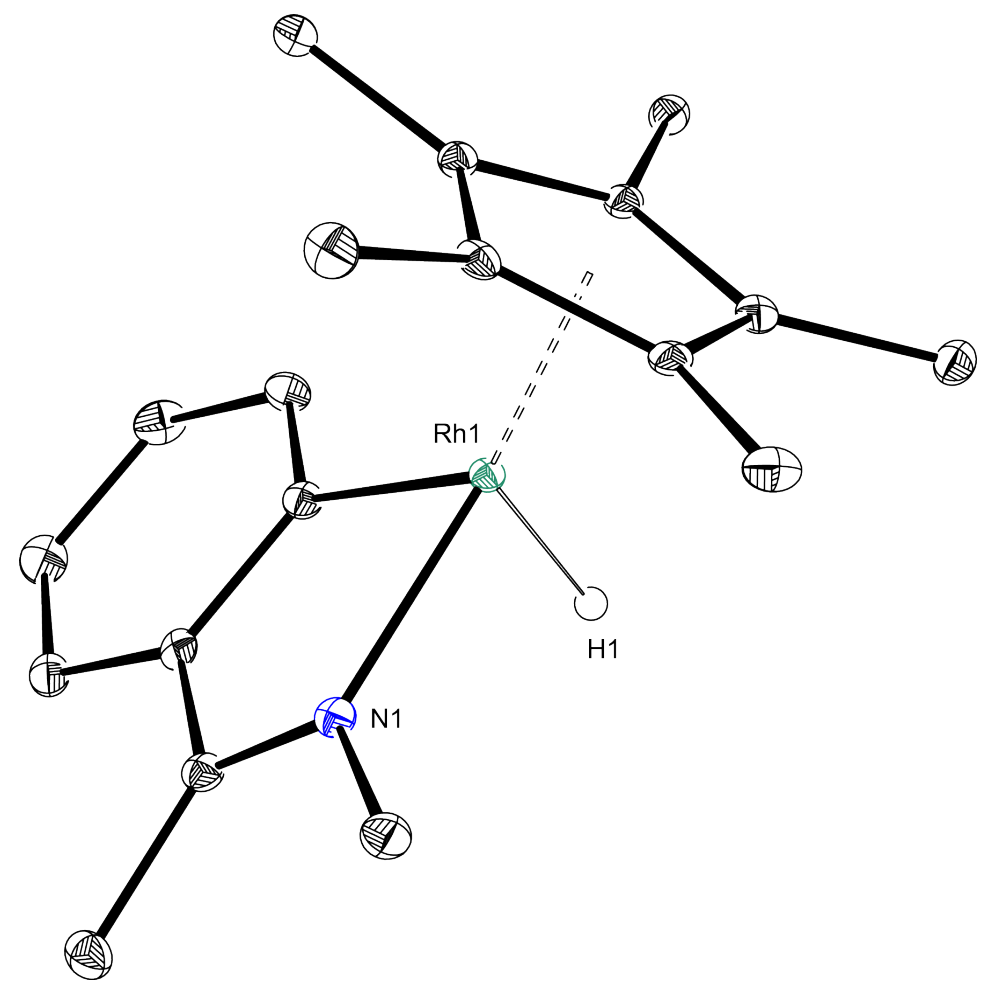

Table S2

Experimental details

\begin{tabular}{|l|l|}
\hline Crystal data & $\mathrm{C}_{76} \mathrm{H}_{104} \mathrm{~N}_{4} \mathrm{Rh}_{4}$ \\
\hline Chemical formula & 1485.27 \\
\hline$M_{\mathrm{r}}$ & Monoclinic, $P 2_{1} / c$ \\
\hline Crystal system, space group & 100 \\
\hline Temperature $(\mathrm{K})$ & $12.8238(7), 7.5442(4), 17.2155(10)$ \\
\hline$a, b, c(\AA)$ & $102.900(2)$ \\
\hline$\beta\left({ }^{\circ}\right)$ & $1623.48(16)$ \\
\hline$V\left(\AA^{3}\right)$ & 1 \\
\hline$Z$ & Mo $K \alpha$ \\
\hline Radiation type & 1.05 \\
\hline$\mu\left(\mathrm{mm}^{-1}\right)$ & $0.10 \times 0.09 \times 0.06$ \\
\hline Crystal size $(\mathrm{mm})$ & \\
\hline & \\
\hline Data collection & \\
\hline
\end{tabular}




\begin{tabular}{|l|l|}
\hline Diffractometer & Bruker D8 Venture Photon 100 CMOS \\
\hline Absorption correction & $\begin{array}{l}\text { Multi-scan } \\
\text { TWINABS BRUKER AXS }\end{array}$ \\
\hline$T_{\min }, T_{\max }$ & $0.716,0.746$ \\
\hline $\begin{array}{l}\text { No. of measured, independent and } \\
\text { observed }[I>2 \sigma(I)] \text { reflections }\end{array}$ & $51430,4165,3015$ \\
\hline$R_{\text {int }}$ & 0.106 \\
\hline$(\sin \theta / \lambda)_{\max }\left(\AA^{-1}\right)$ & 0.676 \\
\hline & \\
\hline Refinement & \\
\hline$R\left[F^{2}>2 \sigma\left(F^{2}\right)\right], w R\left(F^{2}\right), S$ & $0.062,0.119,1.07$ \\
\hline No. of reflections & 4165 \\
\hline No. of parameters & 194 \\
\hline H-atom treatment & $\begin{array}{l}\text { H atoms treated by a mixture of independent } \\
\text { and constrained refinement }\end{array}$ \\
\hline & $\begin{array}{l}w=1 /\left[\sigma^{2}\left(F_{\mathrm{o}}{ }^{2}\right)+(0.0292 P)^{2}+12.5981 P\right] \\
\text { where } P=\left(F_{\mathrm{o}}{ }^{2}+2 F_{\mathrm{c}}{ }^{2}\right) / 3\end{array}$ \\
\hline$\left.\Delta\rangle_{\max }, \Delta\right\rangle_{\min }\left(\mathrm{e} \AA^{-3}\right)$ & $1.37,-0.89$ \\
\hline & \\
\hline
\end{tabular}

Computer programs: Bruker APEX3, Bruker SAINT, SHELXT 2014/5 (Sheldrick, 2014), SHELXL2018/3 (Sheldrick, 2018).

Document origin: publCIF [Westrip, S. P. (2010). J. Apply. Cryst., 43, 920-925].

full crystallographic data

Computing details

Data collection: Bruker APEX3; cell refinement: Bruker SAINT; data reduction: Bruker SAINT; program(s) used to solve structure: SHELXT 2014/5 (Sheldrick, 2014); program(s) used to refine structure: SHELXL2018/3 (Sheldrick, 2018).

(SMK13)

Crystal data

\begin{tabular}{|l|l|}
\hline $\mathrm{C}_{76} \mathrm{H}_{104} \mathrm{~N}_{4} \mathrm{Rh}_{4}$ & $F(000)=768$ \\
\hline$M_{r}=1485.27$ & $D_{\mathrm{x}}=1.519 \mathrm{Mg} \mathrm{m}^{-3}$ \\
\hline Monoclinic, $P 2_{1} / c$ & Mo $K \alpha$ radiation, $\lambda=0.71073 \AA$ \\
\hline$a=12.8238(7) \AA$ & Cell parameters from 9968 reflections \\
\hline$b=7.5442(4) \AA$ & $\theta=2.4-28.7^{\circ}$ \\
\hline
\end{tabular}




\begin{tabular}{|l|l|}
\hline$c=17.2155(10) \AA$ & $\mu=1.05 \mathrm{~mm}^{-1}$ \\
\hline$\beta=102.900(2)^{\circ}$ & $T=100 \mathrm{~K}$ \\
\hline$V=1623.48(16) \AA^{3}$ & Rod, yellow \\
\hline$Z=1$ & $0.10 \times 0.09 \times 0.06 \mathrm{~mm}$ \\
\hline
\end{tabular}

\section{Data collection}

\begin{tabular}{|l|l|}
\hline $\begin{array}{l}\text { Bruker D8 Venture Photon } 100 \text { CMOS } \\
\text { diffractometer }\end{array}$ & 3015 reflections with $I>2 \sigma(I)$ \\
\hline Radiation source: $I \mu S$ microfocus source & $R_{\text {int }}=0.106$ \\
\hline phi and $\omega$ scans & $\theta_{\max }=28.7^{\circ}, \theta_{\min }=2.6^{\circ}$ \\
\hline $\begin{array}{l}\text { Absorption correction: multi-scan } \\
\text { TWINABS BRUKER AXS }\end{array}$ & $h=-17 \rightarrow 15$ \\
\hline$T_{\min }=0.716, T_{\max }=0.746$ & $k=-10 \rightarrow 10$ \\
\hline 51430 measured reflections & $l=-23 \rightarrow 23$ \\
\hline 4165 independent reflections & \\
\hline
\end{tabular}

\section{Refinement}

\begin{tabular}{|l|l|}
\hline Refinement on $F^{2}$ & 0 restraints \\
\hline Least-squares matrix: full & Hydrogen site location: mixed \\
\hline$R\left[F^{2}>2 \sigma\left(F^{2}\right)\right]=0.062$ & $\begin{array}{l}\text { H atoms treated by a mixture of independent } \\
\text { and constrained refinement }\end{array}$ \\
\hline$w R\left(F^{2}\right)=0.119$ & $\begin{array}{l}w=1 /\left[\sigma^{2}\left(F_{\mathrm{o}}^{2}\right)+(0.0292 P)^{2}+12.5981 P\right] \\
\text { where } P=\left(F_{\mathrm{o}}{ }^{2}+2 F_{\mathrm{c}}{ }^{2}\right) / 3\end{array}$ \\
\hline$S=1.07$ & $(\Delta / \sigma)_{\max }=0.001$ \\
\hline 4165 reflections & $\Delta\rangle_{\max }=1.37 \mathrm{e} \AA^{-3}$ \\
\hline 194 parameters & $\Delta\rangle_{\min }=-0.89 \mathrm{e} \AA^{-3}$ \\
\hline
\end{tabular}

\section{Special details}

Experimental. Collected, solved and refined by Hongyu Zhong

Geometry. All esds (except the esd in the dihedral angle between two 1.s. planes) are estimated using the full covariance matrix. The cell esds are taken into account individually in the estimation of esds in distances, angles and torsion angles; correlations between esds in cell parameters are only used when they are defined by crystal symmetry. An approximate (isotropic) treatment of cell esds is used for estimating esds involving l.s. planes.

Fractional atomic coordinates and isotropic or equivalent isotropic displacement parameters $\left(\AA^{2}\right)$ for (SMK13)

\begin{tabular}{|l|l|l|l|l|}
\hline & $x$ & $y$ & $z$ & $U_{\text {iso }} * / U_{\text {eq }}$ \\
\hline
\end{tabular}




\begin{tabular}{|c|c|c|c|c|}
\hline $\mathrm{C} 1$ & $0.5823(4)$ & $0.7766(6)$ & $0.6934(3)$ & $0.0131(10)$ \\
\hline $\mathrm{C} 2$ & $0.6750(4)$ & $0.8882(7)$ & $0.6989(3)$ & $0.0131(9)$ \\
\hline C3 & $0.6916(4)$ & $0.9103(6)$ & 0.6207 (3) & $0.0138(10)$ \\
\hline $\mathrm{C} 4$ & $0.6070(4)$ & $0.8195(6)$ & $0.5656(3)$ & $0.0137(10)$ \\
\hline C5 & $0.5385(4)$ & $0.7420(6)$ & $0.6096(3)$ & $0.0132(10)$ \\
\hline C6 & $0.5261(4)$ & $0.7362(7)$ & $0.7591(3)$ & $0.0158(11)$ \\
\hline H6A & 0.466756 & 0.658511 & 0.739360 & $0.024 *$ \\
\hline H6B & 0.575051 & 0.679750 & 0.802341 & $0.024 *$ \\
\hline $\mathrm{H} 6 \mathrm{C}$ & 0.500451 & 0.844369 & 0.777522 & $0.024 *$ \\
\hline C7 & $0.7399(4)$ & $0.9689(7)$ & $0.7735(3)$ & $0.0168(11)$ \\
\hline H7A & 0.711560 & 0.932498 & 0.818119 & $0.025^{*}$ \\
\hline H7B & 0.812811 & 0.930391 & 0.781230 & $0.025^{*}$ \\
\hline $\mathrm{H} 7 \mathrm{C}$ & 0.736877 & 1.095767 & 0.769082 & $0.025^{*}$ \\
\hline $\mathrm{C} 8$ & $0.7756(4)$ & $1.0237(7)$ & $0.5973(3)$ & $0.0205(12)$ \\
\hline H8A & 0.769545 & 1.015303 & 0.540778 & $0.031 *$ \\
\hline H8B & 0.765924 & 1.144757 & 0.611360 & $0.031 *$ \\
\hline $\mathrm{H} 8 \mathrm{C}$ & 0.845206 & 0.983749 & 0.624655 & $0.031^{*}$ \\
\hline C9 & $0.5877(4)$ & $0.8299(7)$ & $0.4763(3)$ & $0.0190(11)$ \\
\hline H9A & 0.646755 & 0.889269 & 0.461678 & $0.029 *$ \\
\hline H9B & 0.581133 & 0.712382 & 0.454394 & $0.029^{*}$ \\
\hline H9C & 0.523036 & 0.894792 & 0.455822 & $0.029^{*}$ \\
\hline $\mathrm{C} 10$ & $0.4335(4)$ & $0.6520(7)$ & $0.5772(3)$ & $0.0169(11)$ \\
\hline $\mathrm{H} 10 \mathrm{~A}$ & 0.403851 & 0.611512 & 0.620580 & $0.025^{*}$ \\
\hline H10B & 0.385019 & 0.734188 & 0.545232 & $0.025^{*}$ \\
\hline H10C & 0.444455 & 0.552633 & 0.545130 & $0.025^{*}$ \\
\hline C11 & $0.7926(4)$ & $0.4990(7)$ & $0.7462(3)$ & $0.0138(10)$ \\
\hline C12 & $0.7761(4)$ & $0.4895(7)$ & $0.8243(3)$ & $0.0180(11)$ \\
\hline H12 & 0.714142 & 0.537414 & 0.835253 & $0.022 *$ \\
\hline C13 & 0.8505 (4) & $0.4099(7)$ & $0.8850(3)$ & $0.0218(12)$ \\
\hline H13 & 0.838932 & 0.407973 & 0.936441 & $0.026^{*}$ \\
\hline $\mathrm{C} 14$ & $0.9430(4)$ & $0.3325(7)$ & $0.8696(3)$ & $0.0230(12)$ \\
\hline H14 & 0.992718 & 0.280187 & 0.910864 & $0.028^{*}$ \\
\hline C15 & $0.9607(4)$ & $0.3334(7)$ & $0.7939(3)$ & $0.0178(11)$ \\
\hline H15 & 1.021572 & 0.280055 & 0.783500 & $0.021^{*}$ \\
\hline $\mathrm{C} 16$ & $0.8863(4)$ & $0.4155(6)$ & $0.7322(3)$ & $0.0143(10)$ \\
\hline $\mathrm{C} 17$ & $0.8965(4)$ & $0.4248(6)$ & $0.6499(3)$ & $0.0146(11)$ \\
\hline C18 & $0.9864(4)$ & $0.3338(7)$ & $0.6210(3)$ & 0.0209 (12) \\
\hline
\end{tabular}




\begin{tabular}{|l|l|l|l|l|}
\hline H18A & 1.032797 & 0.274919 & 0.664955 & $0.031^{*}$ \\
\hline H18B & 0.956858 & 0.248334 & 0.580799 & $0.031^{*}$ \\
\hline H18C & 1.026528 & 0.420443 & 0.599069 & $0.031^{*}$ \\
\hline C19 & $0.8205(4)$ & $0.5360(8)$ & $0.5182(3)$ & $0.0192(11)$ \\
\hline H19A & 0.759768 & 0.606131 & 0.493245 & $0.029^{*}$ \\
\hline H19B & 0.884957 & 0.594218 & 0.512548 & $0.029^{*}$ \\
\hline H19C & 0.815977 & 0.421579 & 0.493330 & $0.029^{*}$ \\
\hline N1 & $0.8217(3)$ & $0.5149(5)$ & $0.6032(2)$ & $0.0134(9)$ \\
\hline Rh1 & $0.69982(3)$ & $0.61650(5)$ & $0.65104(2)$ & $0.01058(12)$ \\
\hline H1 & $0.652(4)$ & $0.428(7)$ & $0.644(3)$ & $0.023(15)^{*}$ \\
\hline
\end{tabular}

Atomic displacement parameters $\left(\AA^{2}\right)$ for (SMK13)

\begin{tabular}{|l|l|l|l|l|l|l|}
\hline & $U^{11}$ & $U^{22}$ & $U^{33}$ & $U^{12}$ & $U^{13}$ & $U^{23}$ \\
\hline C1 & $0.015(2)$ & $0.010(2)$ & $0.014(3)$ & $0.0025(19)$ & $0.001(2)$ & $-0.001(2)$ \\
\hline C2 & $0.013(2)$ & $0.011(2)$ & $0.016(2)$ & $0.000(2)$ & $0.0038(18)$ & $0.000(2)$ \\
\hline C3 & $0.013(2)$ & $0.008(2)$ & $0.021(3)$ & $0.0017(19)$ & $0.006(2)$ & $0.003(2)$ \\
\hline C4 & $0.017(2)$ & $0.008(2)$ & $0.015(3)$ & $0.0038(19)$ & $0.001(2)$ & $-0.0006(19)$ \\
\hline C5 & $0.015(2)$ & $0.007(2)$ & $0.017(3)$ & $0.0020(19)$ & $0.003(2)$ & $0.003(2)$ \\
\hline C6 & $0.016(2)$ & $0.015(3)$ & $0.016(3)$ & $-0.001(2)$ & $0.003(2)$ & $-0.001(2)$ \\
\hline C7 & $0.016(3)$ & $0.015(3)$ & $0.019(3)$ & $0.002(2)$ & $0.003(2)$ & $-0.001(2)$ \\
\hline C8 & $0.021(3)$ & $0.018(3)$ & $0.024(3)$ & $-0.003(2)$ & $0.009(2)$ & $0.003(2)$ \\
\hline C9 & $0.026(3)$ & $0.016(3)$ & $0.016(3)$ & $0.004(2)$ & $0.007(2)$ & $0.001(2)$ \\
\hline C10 & $0.018(3)$ & $0.016(3)$ & $0.016(3)$ & $-0.001(2)$ & $0.001(2)$ & $-0.001(2)$ \\
\hline C11 & $0.016(2)$ & $0.010(2)$ & $0.016(3)$ & $0.000(2)$ & $0.003(2)$ & $0.001(2)$ \\
\hline C12 & $0.020(3)$ & $0.015(3)$ & $0.019(3)$ & $0.003(2)$ & $0.006(2)$ & $0.002(2)$ \\
\hline C13 & $0.032(3)$ & $0.021(3)$ & $0.012(3)$ & $0.002(2)$ & $0.004(2)$ & $0.002(2)$ \\
\hline C14 & $0.025(3)$ & $0.016(3)$ & $0.026(3)$ & $0.002(2)$ & $0.001(2)$ & $0.004(2)$ \\
\hline C15 & $0.016(3)$ & $0.012(3)$ & $0.024(3)$ & $0.000(2)$ & $0.002(2)$ & $0.001(2)$ \\
\hline C16 & $0.014(2)$ & $0.007(2)$ & $0.022(3)$ & $-0.0042(19)$ & $0.003(2)$ & $0.001(2)$ \\
\hline C17 & $0.016(2)$ & $0.011(3)$ & $0.017(3)$ & $-0.0017(19)$ & $0.005(2)$ & $-0.001(2)$ \\
\hline C18 & $0.018(3)$ & $0.017(3)$ & $0.030(3)$ & $-0.001(2)$ & $0.009(2)$ & $-0.001(2)$ \\
\hline C19 & $0.019(3)$ & $0.023(3)$ & $0.017(3)$ & $-0.001(2)$ & $0.008(2)$ & $-0.002(2)$ \\
\hline N1 & $0.014(2)$ & $0.009(2)$ & $0.018(2)$ & $-0.0032(17)$ & $0.0056(17)$ & $-0.0003(17)$ \\
\hline Rh1 & $0.01144(18)$ & $0.00808(18)$ & $0.01230(19)$ & $0.00028(17)$ & $0.00282(13)$ & $0.00060(17)$ \\
\hline
\end{tabular}

Geometric parameters $\left(\AA,{ }^{\circ}\right)$ for (SMK13) 


\begin{tabular}{|c|c|c|c|}
\hline $\mathrm{C} 1-\mathrm{C} 2$ & $1.443(7)$ & $\mathrm{C} 10-\mathrm{H} 10 \mathrm{~A}$ & 0.9600 \\
\hline $\mathrm{C} 1-\mathrm{C} 5$ & $1.450(7)$ & C10-H10B & 0.9600 \\
\hline $\mathrm{C} 1-\mathrm{C} 6$ & $1.502(7)$ & $\mathrm{C} 10-\mathrm{H} 10 \mathrm{C}$ & 0.9600 \\
\hline $\mathrm{C} 1-\mathrm{Rh} 1$ & $2.180(5)$ & $\mathrm{C} 11-\mathrm{C} 12$ & $1.408(7)$ \\
\hline $\mathrm{C} 2-\mathrm{C} 3$ & $1.420(7)$ & $\mathrm{C} 11-\mathrm{C} 16$ & $1.425(7)$ \\
\hline $\mathrm{C} 2-\mathrm{C} 7$ & $1.495(7)$ & C11-Rh1 & $2.003(5)$ \\
\hline $\mathrm{C} 2-\mathrm{Rh} 1$ & $2.258(5)$ & $\mathrm{C} 12-\mathrm{C} 13$ & $1.385(7)$ \\
\hline $\mathrm{C} 3-\mathrm{C} 4$ & $1.444(7)$ & $\mathrm{C} 12-\mathrm{H} 12$ & 0.9300 \\
\hline $\mathrm{C} 3-\mathrm{C} 8$ & $1.500(7)$ & $\mathrm{C} 13-\mathrm{C} 14$ & $1.400(8)$ \\
\hline $\mathrm{C} 3-\mathrm{Rh} 1$ & $2.274(5)$ & C13-H13 & 0.9300 \\
\hline $\mathrm{C} 4-\mathrm{C} 5$ & $1.409(7)$ & $\mathrm{C} 14-\mathrm{C} 15$ & $1.372(8)$ \\
\hline $\mathrm{C} 4-\mathrm{C} 9$ & $1.503(7)$ & C14-H14 & 0.9300 \\
\hline $\mathrm{C} 4-\mathrm{Rh} 1$ & $2.268(5)$ & $\mathrm{C} 15-\mathrm{C} 16$ & $1.402(7)$ \\
\hline $\mathrm{C} 5-\mathrm{C} 10$ & $1.500(7)$ & C15-H15 & 0.9300 \\
\hline $\mathrm{C} 5-\mathrm{Rh} 1$ & $2.242(5)$ & $\mathrm{C} 16-\mathrm{C} 17$ & $1.454(7)$ \\
\hline C6-H6A & 0.9600 & $\mathrm{C} 17-\mathrm{N} 1$ & $1.298(6)$ \\
\hline C6-H6B & 0.9600 & $\mathrm{C} 17-\mathrm{C} 18$ & $1.518(7)$ \\
\hline $\mathrm{C} 6-\mathrm{H} 6 \mathrm{C}$ & 0.9600 & C18-H18A & 0.9600 \\
\hline $\mathrm{C} 7-\mathrm{H} 7 \mathrm{~A}$ & 0.9600 & $\mathrm{C} 18-\mathrm{H} 18 \mathrm{~B}$ & 0.9600 \\
\hline C7-H7B & 0.9600 & $\mathrm{C} 18-\mathrm{H} 18 \mathrm{C}$ & 0.9600 \\
\hline $\mathrm{C} 7-\mathrm{H} 7 \mathrm{C}$ & 0.9600 & $\mathrm{C} 19-\mathrm{N} 1$ & $1.469(6)$ \\
\hline $\mathrm{C} 8-\mathrm{H} 8 \mathrm{~A}$ & 0.9600 & C19-H19A & 0.9600 \\
\hline $\mathrm{C} 8-\mathrm{H} 8 \mathrm{~B}$ & 0.9600 & $\mathrm{C} 19-\mathrm{H} 19 \mathrm{~B}$ & 0.9600 \\
\hline $\mathrm{C} 8-\mathrm{H} 8 \mathrm{C}$ & 0.9600 & C19-H19C & 0.9600 \\
\hline C9-H9A & 0.9600 & $\mathrm{~N} 1-\mathrm{Rh} 1$ & $2.070(4)$ \\
\hline C9-H9B & 0.9600 & $\mathrm{Rh} 1-\mathrm{H} 1$ & $1.55(6)$ \\
\hline $\mathrm{C} 9-\mathrm{H} 9 \mathrm{C}$ & 0.9600 & & \\
\hline $\mathrm{C} 2-\mathrm{C} 1-\mathrm{C} 5$ & $107.5(4)$ & $\mathrm{C} 16-\mathrm{C} 11-\mathrm{Rh} 1$ & $115.3(4)$ \\
\hline $\mathrm{C} 2-\mathrm{C} 1-\mathrm{C} 6$ & $126.6(4)$ & $\mathrm{C} 13-\mathrm{C} 12-\mathrm{C} 11$ & $121.2(5)$ \\
\hline $\mathrm{C} 5-\mathrm{C} 1-\mathrm{C} 6$ & $124.6(4)$ & $\mathrm{C} 13-\mathrm{C} 12-\mathrm{H} 12$ & 119.4 \\
\hline $\mathrm{C} 2-\mathrm{C} 1-\mathrm{Rh} 1$ & $74.0(3)$ & $\mathrm{C} 11-\mathrm{C} 12-\mathrm{H} 12$ & 119.4 \\
\hline $\mathrm{C} 5-\mathrm{C} 1-\mathrm{Rh} 1$ & $73.2(3)$ & $\mathrm{C} 12-\mathrm{C} 13-\mathrm{C} 14$ & $120.5(5)$ \\
\hline $\mathrm{C} 6-\mathrm{C} 1-\mathrm{Rh} 1$ & $128.5(3)$ & $\mathrm{C} 12-\mathrm{C} 13-\mathrm{H} 13$ & 119.7 \\
\hline $\mathrm{C} 3-\mathrm{C} 2-\mathrm{C} 1$ & $107.6(4)$ & $\mathrm{C} 14-\mathrm{C} 13-\mathrm{H} 13$ & 119.7 \\
\hline $\mathrm{C} 3-\mathrm{C} 2-\mathrm{C} 7$ & $126.5(4)$ & $\mathrm{C} 15-\mathrm{C} 14-\mathrm{C} 13$ & $120.3(5)$ \\
\hline $\mathrm{C} 1-\mathrm{C} 2-\mathrm{C} 7$ & $125.9(4)$ & $\mathrm{C} 15-\mathrm{C} 14-\mathrm{H} 14$ & 119.8 \\
\hline
\end{tabular}




\begin{tabular}{|c|c|c|c|}
\hline $\mathrm{C} 3-\mathrm{C} 2-\mathrm{Rh} 1$ & $72.4(3)$ & $\mathrm{C} 13-\mathrm{C} 14-\mathrm{H} 14$ & 119.8 \\
\hline $\mathrm{C} 1-\mathrm{C} 2-\mathrm{Rh} 1$ & $68.1(3)$ & $\mathrm{C} 14-\mathrm{C} 15-\mathrm{C} 16$ & $119.5(5)$ \\
\hline $\mathrm{C} 7-\mathrm{C} 2-\mathrm{Rh} 1$ & $125.9(3)$ & $\mathrm{C} 14-\mathrm{C} 15-\mathrm{H} 15$ & 120.3 \\
\hline $\mathrm{C} 2-\mathrm{C} 3-\mathrm{C} 4$ & $108.5(4)$ & $\mathrm{C} 16-\mathrm{C} 15-\mathrm{H} 15$ & 120.3 \\
\hline $\mathrm{C} 2-\mathrm{C} 3-\mathrm{C} 8$ & $126.2(5)$ & $\mathrm{C} 15-\mathrm{C} 16-\mathrm{C} 11$ & $121.7(5)$ \\
\hline $\mathrm{C} 4-\mathrm{C} 3-\mathrm{C} 8$ & $125.0(5)$ & $\mathrm{C} 15-\mathrm{C} 16-\mathrm{C} 17$ & $124.4(5)$ \\
\hline $\mathrm{C} 2-\mathrm{C} 3-\mathrm{Rh} 1$ & $71.1(3)$ & $\mathrm{C} 11-\mathrm{C} 16-\mathrm{C} 17$ & $114.0(4)$ \\
\hline $\mathrm{C} 4-\mathrm{C} 3-\mathrm{Rh} 1$ & $71.2(3)$ & $\mathrm{N} 1-\mathrm{C} 17-\mathrm{C} 16$ & $114.5(4)$ \\
\hline $\mathrm{C} 8-\mathrm{C} 3-\mathrm{Rh} 1$ & $128.2(3)$ & $\mathrm{N} 1-\mathrm{C} 17-\mathrm{C} 18$ & $123.0(5)$ \\
\hline $\mathrm{C} 5-\mathrm{C} 4-\mathrm{C} 3$ & $108.1(4)$ & $\mathrm{C} 16-\mathrm{C} 17-\mathrm{C} 18$ & $122.5(5)$ \\
\hline $\mathrm{C} 5-\mathrm{C} 4-\mathrm{C} 9$ & $126.3(5)$ & $\mathrm{C} 17-\mathrm{C} 18-\mathrm{H} 18 \mathrm{~A}$ & 109.5 \\
\hline $\mathrm{C} 3-\mathrm{C} 4-\mathrm{C} 9$ & $125.0(4)$ & $\mathrm{C} 17-\mathrm{C} 18-\mathrm{H} 18 \mathrm{~B}$ & 109.5 \\
\hline $\mathrm{C} 5-\mathrm{C} 4-\mathrm{Rh} 1$ & $70.8(3)$ & $\mathrm{H} 18 \mathrm{~A}-\mathrm{C} 18-\mathrm{H} 18 \mathrm{~B}$ & 109.5 \\
\hline $\mathrm{C} 3-\mathrm{C} 4-\mathrm{Rh} 1$ & $71.7(3)$ & $\mathrm{C} 17-\mathrm{C} 18-\mathrm{H} 18 \mathrm{C}$ & 109.5 \\
\hline $\mathrm{C} 9-\mathrm{C} 4-\mathrm{Rh} 1$ & $129.9(3)$ & $\mathrm{H} 18 \mathrm{~A}-\mathrm{C} 18-\mathrm{H} 18 \mathrm{C}$ & 109.5 \\
\hline $\mathrm{C} 4-\mathrm{C} 5-\mathrm{C} 1$ & $108.1(4)$ & $\mathrm{H} 18 \mathrm{~B}-\mathrm{C} 18-\mathrm{H} 18 \mathrm{C}$ & 109.5 \\
\hline $\mathrm{C} 4-\mathrm{C} 5-\mathrm{C} 10$ & $127.1(5)$ & $\mathrm{N} 1-\mathrm{C} 19-\mathrm{H} 19 \mathrm{~A}$ & 109.5 \\
\hline $\mathrm{C} 1-\mathrm{C} 5-\mathrm{C} 10$ & $124.6(4)$ & $\mathrm{N} 1-\mathrm{C} 19-\mathrm{H} 19 \mathrm{~B}$ & 109.5 \\
\hline $\mathrm{C} 4-\mathrm{C} 5-\mathrm{Rh} 1$ & $72.8(3)$ & $\mathrm{H} 19 \mathrm{~A}-\mathrm{C} 19-\mathrm{H} 19 \mathrm{~B}$ & 109.5 \\
\hline $\mathrm{C} 1-\mathrm{C} 5-\mathrm{Rh} 1$ & $68.6(3)$ & $\mathrm{N} 1-\mathrm{C} 19-\mathrm{H} 19 \mathrm{C}$ & 109.5 \\
\hline $\mathrm{C} 10-\mathrm{C} 5-\mathrm{Rh} 1$ & $127.9(3)$ & $\mathrm{H} 19 \mathrm{~A}-\mathrm{C} 19-\mathrm{H} 19 \mathrm{C}$ & 109.5 \\
\hline $\mathrm{C} 1-\mathrm{C} 6-\mathrm{H} 6 \mathrm{~A}$ & 109.5 & $\mathrm{H} 19 \mathrm{~B}-\mathrm{C} 19-\mathrm{H} 19 \mathrm{C}$ & 109.5 \\
\hline $\mathrm{C} 1-\mathrm{C} 6-\mathrm{H} 6 \mathrm{~B}$ & 109.5 & $\mathrm{C} 17-\mathrm{N} 1-\mathrm{C} 19$ & $121.0(4)$ \\
\hline $\mathrm{H} 6 \mathrm{~A}-\mathrm{C} 6-\mathrm{H} 6 \mathrm{~B}$ & 109.5 & $\mathrm{C} 17-\mathrm{N} 1-\mathrm{Rh} 1$ & $117.9(3)$ \\
\hline $\mathrm{C} 1-\mathrm{C} 6-\mathrm{H} 6 \mathrm{C}$ & 109.5 & $\mathrm{C} 19-\mathrm{N} 1-\mathrm{Rh} 1$ & $121.0(3)$ \\
\hline $\mathrm{H} 6 \mathrm{~A}-\mathrm{C} 6-\mathrm{H} 6 \mathrm{C}$ & 109.5 & $\mathrm{C} 11-\mathrm{Rh} 1-\mathrm{N} 1$ & $78.36(18)$ \\
\hline $\mathrm{H} 6 \mathrm{~B}-\mathrm{C} 6-\mathrm{H} 6 \mathrm{C}$ & 109.5 & $\mathrm{C} 11-\mathrm{Rh} 1-\mathrm{C} 1$ & $107.46(19)$ \\
\hline $\mathrm{C} 2-\mathrm{C} 7-\mathrm{H} 7 \mathrm{~A}$ & 109.5 & $\mathrm{~N} 1-\mathrm{Rh} 1-\mathrm{C} 1$ & $168.01(17)$ \\
\hline $\mathrm{C} 2-\mathrm{C} 7-\mathrm{H} 7 \mathrm{~B}$ & 109.5 & $\mathrm{C} 11-\mathrm{Rh} 1-\mathrm{C} 5$ & $141.62(19)$ \\
\hline $\mathrm{H} 7 \mathrm{~A}-\mathrm{C} 7-\mathrm{H} 7 \mathrm{~B}$ & 109.5 & $\mathrm{~N} 1-\mathrm{Rh} 1-\mathrm{C} 5$ & $139.06(17)$ \\
\hline $\mathrm{C} 2-\mathrm{C} 7-\mathrm{H} 7 \mathrm{C}$ & 109.5 & $\mathrm{C} 1-\mathrm{Rh} 1-\mathrm{C} 5$ & $38.23(18)$ \\
\hline $\mathrm{H} 7 \mathrm{~A}-\mathrm{C} 7-\mathrm{H} 7 \mathrm{C}$ & 109.5 & $\mathrm{C} 11-\mathrm{Rh} 1-\mathrm{C} 2$ & $102.07(19)$ \\
\hline $\mathrm{H} 7 \mathrm{~B}-\mathrm{C} 7-\mathrm{H} 7 \mathrm{C}$ & 109.5 & $\mathrm{~N} 1-\mathrm{Rh} 1-\mathrm{C} 2$ & $131.44(16)$ \\
\hline $\mathrm{C} 3-\mathrm{C} 8-\mathrm{H} 8 \mathrm{~A}$ & 109.5 & $\mathrm{C} 1-\mathrm{Rh} 1-\mathrm{C} 2$ & $37.89(17)$ \\
\hline $\mathrm{C} 3-\mathrm{C} 8-\mathrm{H} 8 \mathrm{~B}$ & 109.5 & $\mathrm{C} 5-\mathrm{Rh} 1-\mathrm{C} 2$ & $62.46(17)$ \\
\hline $\mathrm{H} 8 \mathrm{~A}-\mathrm{C} 8-\mathrm{H} 8 \mathrm{~B}$ & 109.5 & $\mathrm{C} 11-\mathrm{Rh} 1-\mathrm{C} 4$ & $163.49(19)$ \\
\hline $\mathrm{C} 3-\mathrm{C} 8-\mathrm{H} 8 \mathrm{C}$ & 109.5 & $\mathrm{~N} 1-\mathrm{Rh} 1-\mathrm{C} 4$ & 109.01 (17) \\
\hline
\end{tabular}




\begin{tabular}{|c|c|c|c|}
\hline $\mathrm{H} 8 \mathrm{~A}-\mathrm{C} 8-\mathrm{H} 8 \mathrm{C}$ & 109.5 & $\mathrm{C} 1-\mathrm{Rh} 1-\mathrm{C} 4$ & $62.67(18)$ \\
\hline $\mathrm{H} 8 \mathrm{~B}-\mathrm{C} 8-\mathrm{H} 8 \mathrm{C}$ & 109.5 & $\mathrm{C} 5-\mathrm{Rh} 1-\mathrm{C} 4$ & $36.41(17)$ \\
\hline $\mathrm{C} 4-\mathrm{C} 9-\mathrm{H} 9 \mathrm{~A}$ & 109.5 & $\mathrm{C} 2-\mathrm{Rh} 1-\mathrm{C} 4$ & $61.81(17)$ \\
\hline $\mathrm{C} 4-\mathrm{C} 9-\mathrm{H} 9 \mathrm{~B}$ & 109.5 & $\mathrm{C} 11-\mathrm{Rh} 1-\mathrm{C} 3$ & $127.4(2)$ \\
\hline $\mathrm{H} 9 \mathrm{~A}-\mathrm{C} 9-\mathrm{H} 9 \mathrm{~B}$ & 109.5 & $\mathrm{~N} 1-\mathrm{Rh} 1-\mathrm{C} 3$ & $105.65(16)$ \\
\hline $\mathrm{C} 4-\mathrm{C} 9-\mathrm{H} 9 \mathrm{C}$ & 109.5 & $\mathrm{C} 1-\mathrm{Rh} 1-\mathrm{C} 3$ & $62.42(18)$ \\
\hline $\mathrm{H} 9 \mathrm{~A}-\mathrm{C} 9-\mathrm{H} 9 \mathrm{C}$ & 109.5 & $\mathrm{C} 5-\mathrm{Rh} 1-\mathrm{C} 3$ & $61.52(17)$ \\
\hline $\mathrm{H} 9 \mathrm{~B}-\mathrm{C} 9-\mathrm{H} 9 \mathrm{C}$ & 109.5 & $\mathrm{C} 2-\mathrm{Rh} 1-\mathrm{C} 3$ & $36.52(17)$ \\
\hline $\mathrm{C} 5-\mathrm{C} 10-\mathrm{H} 10 \mathrm{~A}$ & 109.5 & $\mathrm{C} 4-\mathrm{Rh} 1-\mathrm{C} 3$ & $37.08(18)$ \\
\hline $\mathrm{C} 5-\mathrm{C} 10-\mathrm{H} 10 \mathrm{~B}$ & 109.5 & $\mathrm{C} 11-\mathrm{Rh} 1-\mathrm{H} 1$ & $79(2)$ \\
\hline $\mathrm{H} 10 \mathrm{~A}-\mathrm{C} 10-\mathrm{H} 10 \mathrm{~B}$ & 109.5 & $\mathrm{~N} 1-\mathrm{Rh} 1-\mathrm{H} 1$ & $87(2)$ \\
\hline $\mathrm{C} 5-\mathrm{C} 10-\mathrm{H} 10 \mathrm{C}$ & 109.5 & $\mathrm{C} 1-\mathrm{Rh} 1-\mathrm{H} 1$ & $104(2)$ \\
\hline $\mathrm{H} 10 \mathrm{~A}-\mathrm{C} 10-\mathrm{H} 10 \mathrm{C}$ & 109.5 & $\mathrm{C} 5-\mathrm{Rh} 1-\mathrm{H} 1$ & $92(2)$ \\
\hline $\mathrm{H} 10 \mathrm{~B}-\mathrm{C} 10-\mathrm{H} 10 \mathrm{C}$ & 109.5 & $\mathrm{C} 2-\mathrm{Rh} 1-\mathrm{H} 1$ & $141(2)$ \\
\hline $\mathrm{C} 12-\mathrm{C} 11-\mathrm{C} 16$ & $116.8(5)$ & $\mathrm{C} 4-\mathrm{Rh} 1-\mathrm{H} 1$ & $115(2)$ \\
\hline $\mathrm{C} 12-\mathrm{C} 11-\mathrm{Rh} 1$ & $128.0(4)$ & C3-Rh1-H1 & $152(2)$ \\
\hline $\mathrm{C} 5-\mathrm{C} 1-\mathrm{C} 2-\mathrm{C} 3$ & $-4.0(5)$ & $\mathrm{C} 3-\mathrm{C} 4-\mathrm{C} 5-\mathrm{Rh} 1$ & $-62.4(3)$ \\
\hline $\mathrm{C} 6-\mathrm{C} 1-\mathrm{C} 2-\mathrm{C} 3$ & $-171.5(5)$ & $\mathrm{C} 9-\mathrm{C} 4-\mathrm{C} 5-\mathrm{Rh} 1$ & $125.9(5)$ \\
\hline $\mathrm{Rh} 1-\mathrm{C} 1-\mathrm{C} 2-\mathrm{C} 3$ & $62.0(3)$ & $\mathrm{C} 2-\mathrm{C} 1-\mathrm{C} 5-\mathrm{C} 4$ & $4.1(5)$ \\
\hline $\mathrm{C} 5-\mathrm{C} 1-\mathrm{C} 2-\mathrm{C} 7$ & $174.6(5)$ & $\mathrm{C} 6-\mathrm{C} 1-\mathrm{C} 5-\mathrm{C} 4$ & $171.9(5)$ \\
\hline $\mathrm{C} 6-\mathrm{C} 1-\mathrm{C} 2-\mathrm{C} 7$ & $7.1(8)$ & $\mathrm{Rh} 1-\mathrm{C} 1-\mathrm{C} 5-\mathrm{C} 4$ & $-62.4(3)$ \\
\hline $\mathrm{Rh} 1-\mathrm{C} 1-\mathrm{C} 2-\mathrm{C} 7$ & $-119.4(5)$ & $\mathrm{C} 2-\mathrm{C} 1-\mathrm{C} 5-\mathrm{C} 10$ & $-171.3(4)$ \\
\hline $\mathrm{C} 5-\mathrm{C} 1-\mathrm{C} 2-\mathrm{Rh} 1$ & $-66.0(3)$ & $\mathrm{C} 6-\mathrm{C} 1-\mathrm{C} 5-\mathrm{C} 10$ & $-3.5(8)$ \\
\hline $\mathrm{C} 6-\mathrm{C} 1-\mathrm{C} 2-\mathrm{Rh} 1$ & $126.4(5)$ & $\mathrm{Rh} 1-\mathrm{C} 1-\mathrm{C} 5-\mathrm{C} 10$ & $122.1(5)$ \\
\hline $\mathrm{C} 1-\mathrm{C} 2-\mathrm{C} 3-\mathrm{C} 4$ & $2.5(5)$ & $\mathrm{C} 2-\mathrm{C} 1-\mathrm{C} 5-\mathrm{Rh} 1$ & $66.5(3)$ \\
\hline $\mathrm{C} 7-\mathrm{C} 2-\mathrm{C} 3-\mathrm{C} 4$ & $-176.2(5)$ & $\mathrm{C} 6-\mathrm{C} 1-\mathrm{C} 5-\mathrm{Rh} 1$ & $-125.6(5)$ \\
\hline $\mathrm{Rh} 1-\mathrm{C} 2-\mathrm{C} 3-\mathrm{C} 4$ & $61.8(3)$ & $\begin{array}{l}\mathrm{C} 16-\mathrm{C} 11-\mathrm{C} 12- \\
\mathrm{C} 13\end{array}$ & $3.1(8)$ \\
\hline $\mathrm{C} 1-\mathrm{C} 2-\mathrm{C} 3-\mathrm{C} 8$ & $176.6(5)$ & $\begin{array}{l}\mathrm{Rh} 1-\mathrm{C} 11-\mathrm{C} 12- \\
\mathrm{C} 13\end{array}$ & $-177.0(4)$ \\
\hline $\mathrm{C} 7-\mathrm{C} 2-\mathrm{C} 3-\mathrm{C} 8$ & $-2.0(8)$ & $\begin{array}{l}\mathrm{C} 11-\mathrm{C} 12-\mathrm{C} 13- \\
\mathrm{C} 14\end{array}$ & $-1.8(8)$ \\
\hline $\mathrm{Rh} 1-\mathrm{C} 2-\mathrm{C} 3-\mathrm{C} 8$ & $-124.1(5)$ & $\begin{array}{l}\mathrm{C} 12-\mathrm{C} 13-\mathrm{C} 14- \\
\mathrm{C} 15\end{array}$ & $-0.5(8)$ \\
\hline $\mathrm{C} 1-\mathrm{C} 2-\mathrm{C} 3-\mathrm{Rh} 1$ & $-59.3(3)$ & $\begin{array}{l}\mathrm{C} 13-\mathrm{C} 14-\mathrm{C} 15- \\
\mathrm{C} 16\end{array}$ & $1.2(8)$ \\
\hline $\mathrm{C} 7-\mathrm{C} 2-\mathrm{C} 3-\mathrm{Rh} 1$ & $122.1(5)$ & $\begin{array}{l}\mathrm{C} 14-\mathrm{C} 15-\mathrm{C} 16- \\
\mathrm{C} 11\end{array}$ & $0.3(8)$ \\
\hline
\end{tabular}




\begin{tabular}{|l|l|l|l|}
\hline $\mathrm{C} 2-\mathrm{C} 3-\mathrm{C} 4-\mathrm{C} 5$ & $0.1(6)$ & $\begin{array}{l}\mathrm{C} 14-\mathrm{C} 15-\mathrm{C} 16- \\
\mathrm{C} 17\end{array}$ & $180.0(5)$ \\
\hline $\mathrm{C} 8-\mathrm{C} 3-\mathrm{C} 4-\mathrm{C} 5$ & $-174.2(5)$ & $\begin{array}{l}\mathrm{C} 12-\mathrm{C} 11-\mathrm{C} 16- \\
\mathrm{C} 15\end{array}$ & $-2.3(7)$ \\
\hline $\mathrm{Rh} 1-\mathrm{C} 3-\mathrm{C} 4-\mathrm{C} 5$ & $61.8(3)$ & $\begin{array}{l}\mathrm{R} 1-\mathrm{C} 11-\mathrm{C} 16- \\
\mathrm{C} 15\end{array}$ & $177.7(4)$ \\
\hline $\mathrm{C} 2-\mathrm{C} 3-\mathrm{C} 4-\mathrm{C} 9$ & $172.0(5)$ & $\begin{array}{l}\mathrm{C} 12-\mathrm{C} 11-\mathrm{C} 16- \\
\mathrm{C} 17\end{array}$ & $177.9(4)$ \\
\hline $\mathrm{C} 8-\mathrm{C} 3-\mathrm{C} 4-\mathrm{C} 9$ & $-2.3(8)$ & $\begin{array}{l}\mathrm{R} 1-\mathrm{C} 11-\mathrm{C} 16- \\
\mathrm{C} 17\end{array}$ & $-2.0(6)$ \\
\hline $\mathrm{Rh} 1-\mathrm{C} 3-\mathrm{C} 4-\mathrm{C} 9$ & $-126.3(5)$ & $\mathrm{C} 15-\mathrm{C} 16-\mathrm{C} 17-\mathrm{N} 1$ & $-176.5(5)$ \\
\hline $\mathrm{C} 2-\mathrm{C} 3-\mathrm{C} 4-\mathrm{Rh} 1$ & $-61.7(3)$ & $\mathrm{C} 11-\mathrm{C} 16-\mathrm{C} 17-\mathrm{N} 1$ & $3.2(6)$ \\
\hline $\mathrm{C} 8-\mathrm{C} 3-\mathrm{C} 4-\mathrm{Rh} 1$ & $124.1(5)$ & $\begin{array}{l}\mathrm{C} 15-\mathrm{C} 16-\mathrm{C} 17- \\
\mathrm{C} 18\end{array}$ & $4.5(8)$ \\
\hline $\mathrm{C} 3-\mathrm{C} 4-\mathrm{C} 5-\mathrm{C} 1$ & $-2.6(5)$ & $\begin{array}{l}\mathrm{C} 11-\mathrm{C} 16-\mathrm{C} 17- \\
\mathrm{C} 18\end{array}$ & $-175.8(4)$ \\
\hline $\mathrm{C} 9-\mathrm{C} 4-\mathrm{C} 5-\mathrm{C} 1$ & $-174.3(5)$ & $\mathrm{C} 16-\mathrm{C} 17-\mathrm{N} 1-\mathrm{C} 19$ & $-179.7(4)$ \\
\hline $\mathrm{R} 1-\mathrm{C} 4-\mathrm{C} 5-\mathrm{C} 1$ & $59.8(3)$ & $\mathrm{C} 18-\mathrm{C} 17-\mathrm{N} 1-\mathrm{C} 19$ & $-0.8(7)$ \\
\hline $\mathrm{C} 3-\mathrm{C} 4-\mathrm{C} 5-\mathrm{C} 10$ & $172.7(5)$ & $\mathrm{C} 16-\mathrm{C} 17-\mathrm{N} 1-\mathrm{Rh} 1$ & $-2.9(6)$ \\
\hline $\mathrm{C} 9-\mathrm{C} 4-\mathrm{C} 5-\mathrm{C} 10$ & $1.0(8)$ & $\mathrm{C} 18-\mathrm{C} 17-\mathrm{N} 1-\mathrm{Rh} 1$ & $176.1(4)$ \\
\hline $\mathrm{R} 1-\mathrm{C} 4-\mathrm{C} 5-\mathrm{C} 10$ & $-124.9(5)$ & & \\
\hline
\end{tabular}

Document origin: publCIF [Westrip, S. P. (2010). J. Apply. Cryst., 43, 920-925].

\section{Compound 3:}




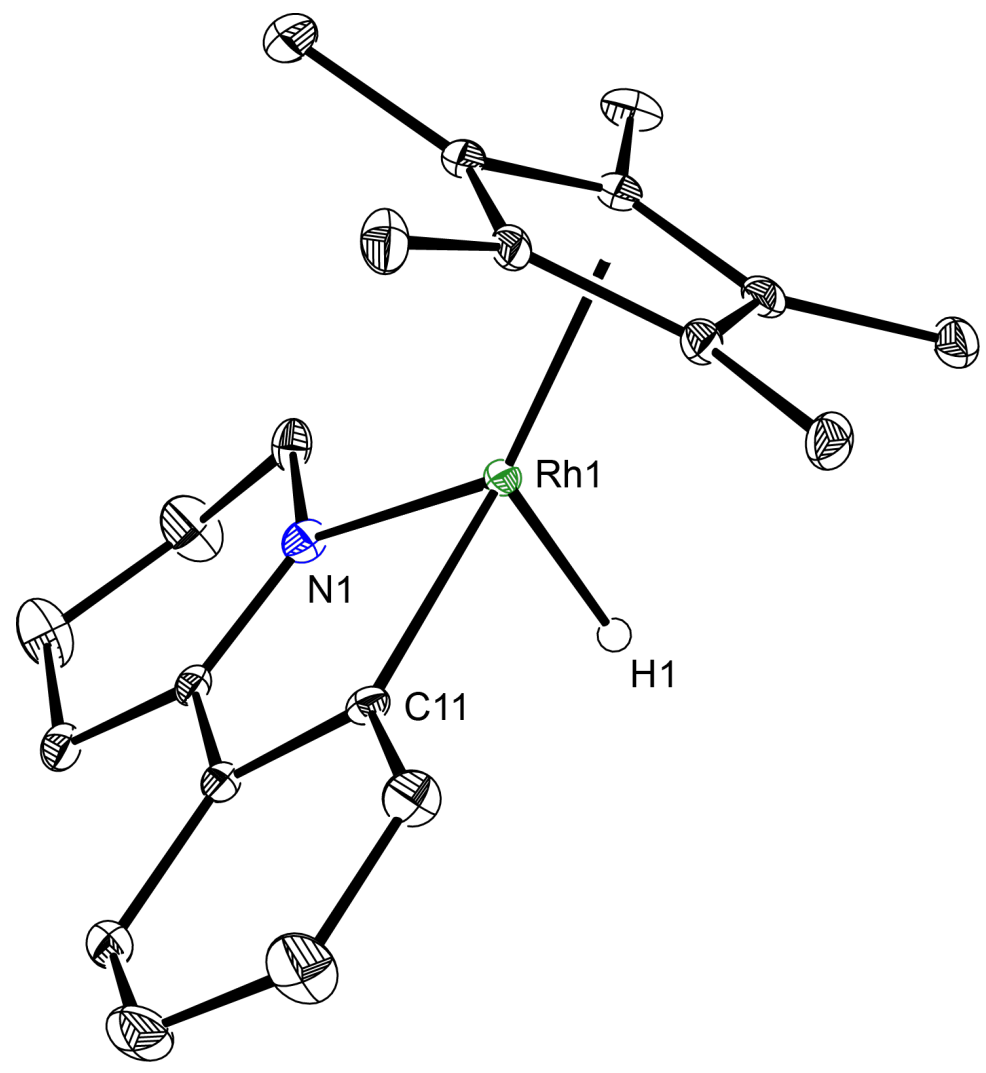

Table S3

Experimental details

\begin{tabular}{|c|c|}
\hline \multicolumn{2}{|l|}{ Crystal data } \\
\hline Chemical formula & $\mathrm{C}_{21} \mathrm{H}_{28} \mathrm{NRh}$ \\
\hline$M_{\mathrm{r}}$ & 397.35 \\
\hline Crystal system, space group & Monoclinic, $P 2_{1} / c$ \\
\hline Temperature (K) & 100 \\
\hline$a, b, c(\AA)$ & $13.5272(6), 7.7023(3), 18.0407(8)$ \\
\hline$\beta\left(^{\circ}\right)$ & $111.505(1)$ \\
\hline$V\left(\AA^{3}\right)$ & $1748.82(13)$ \\
\hline$Z$ & 4 \\
\hline Radiation type & Mo $K \alpha$ \\
\hline$\mu\left(\mathrm{mm}^{-1}\right)$ & 0.98 \\
\hline Crystal size $(\mathrm{mm})$ & $0.21 \times 0.11 \times 0.11$ \\
\hline \multicolumn{2}{|l|}{ Data collection } \\
\hline Diffractometer & Bruker D8 Venture Photon $100 \mathrm{CMOS}$ \\
\hline
\end{tabular}




\begin{tabular}{|l|l|}
\hline Absorption correction & $\begin{array}{l}\text { Multi-scan } \\
\text { TWINABS BRUKER AXS }\end{array}$ \\
\hline$T_{\min }, T_{\max }$ & $0.716,0.746$ \\
\hline $\begin{array}{l}\text { No. of measured, independent and } \\
\text { observed }[I>2 \sigma(I)] \text { reflections }\end{array}$ & $63903,4530,3908$ \\
\hline$R_{\text {int }}$ & 0.047 \\
\hline$(\sin \theta / \lambda)_{\max }\left(\AA^{-1}\right)$ & 0.677 \\
\hline & \\
\hline Refinement & $0.035,0.088,1.08$ \\
\hline$R\left[F^{2}>2 \sigma\left(F^{2}\right)\right], w R\left(F^{2}\right), S$ & 4530 \\
\hline No. of reflections & 212 \\
\hline No. of parameters & $\begin{array}{l}\text { H atoms treated by a mixture of independent } \\
\text { and constrained refinement }\end{array}$ \\
\hline H-atom treatment & $0.66,-0.68$ \\
\hline$\left.\Delta\rangle_{\max }, \Delta\right\rangle_{\min }\left(\mathrm{e} \AA^{-3}\right)$ &
\end{tabular}

Computer programs: Bruker APEX3, Bruker SAINT, SHELXT 2014/5 (Sheldrick, 2014), SHELXL2018/3 (Sheldrick, 2018).

Document origin: publCIF [Westrip, S. P. (2010). J. Apply. Cryst., 43, 920-925].

full crystallographic data

Computing details

Data collection: Bruker APEX3; cell refinement: Bruker SAINT; data reduction: Bruker SAINT; program(s) used to solve structure: SHELXT 2014/5 (Sheldrick, 2014); program(s) used to refine structure: SHELXL2018/3 (Sheldrick, 2018).

\section{(SMK11)}

\section{Crystal data}

\begin{tabular}{|l|l|}
\hline $\mathrm{C}_{21} \mathrm{H}_{28} \mathrm{NRh}$ & $F(000)=824$ \\
\hline$M_{r}=397.35$ & $D_{\mathrm{x}}=1.509 \mathrm{Mg} \mathrm{m}^{-3}$ \\
\hline Monoclinic, $P 2_{1} / c$ & Mo $K \alpha$ radiation, $\lambda=0.71073 \AA$ \\
\hline$a=13.5272(6) \AA$ & Cell parameters from 9628 reflections \\
\hline$b=7.7023(3) \AA$ & $\theta=2.9-28.7^{\circ}$ \\
\hline$c=18.0407(8) \AA$ & $\mu=0.98 \mathrm{~mm}^{-1}$ \\
\hline$\beta=111.505(1)^{\circ}$ & $T=100 \mathrm{~K}$ \\
\hline$V=1748.82(13) \AA^{3}$ & Plate, orange-yellow \\
\hline
\end{tabular}




\begin{tabular}{|l|l|}
\hline$Z=4$ & $0.21 \times 0.11 \times 0.11 \mathrm{~mm}$ \\
\hline
\end{tabular}

\section{Data collection}

\begin{tabular}{|l|l|}
\hline $\begin{array}{l}\text { Bruker D8 Venture Photon } 100 \mathrm{CMOS} \\
\text { diffractometer }\end{array}$ & 3908 reflections with $I>2 \sigma(I)$ \\
\hline Radiation source: $\mathrm{I} \mu \mathrm{S}$ microfocus source & $R_{\text {int }}=0.047$ \\
\hline phi and $\omega$ scans & $\theta_{\max }=28.8^{\circ}, \theta_{\min }=2.4^{\circ}$ \\
\hline $\begin{array}{l}\text { Absorption correction: multi-scan } \\
\text { TWINABS BRUKER AXS }\end{array}$ & $h=-18 \rightarrow 18$ \\
\hline$T_{\min }=0.716, T_{\max }=0.746$ & $k=-10 \rightarrow 10$ \\
\hline 63903 measured reflections & $l=-24 \rightarrow 24$ \\
\hline 4530 independent reflections & \\
\hline
\end{tabular}

\section{Refinement}

\begin{tabular}{|l|l|}
\hline Refinement on $F^{2}$ & 0 restraints \\
\hline Least-squares matrix: full & Hydrogen site location: mixed \\
\hline$R\left[F^{2}>2 \sigma\left(F^{2}\right)\right]=0.035$ & $\begin{array}{l}\text { H atoms treated by a mixture of independent } \\
\text { and constrained refinement }\end{array}$ \\
\hline$w R\left(F^{2}\right)=0.088$ & $\begin{array}{l}w=1 /\left[\sigma^{2}\left(F_{\mathrm{o}}^{2}\right)+(0.0355 P)^{2}+5.0929 P\right] \\
\text { where } P=\left(F_{\mathrm{o}}^{2}+2 F_{\mathrm{c}}^{2}\right) / 3\end{array}$ \\
\hline$S=1.08$ & $(\Delta / \sigma)_{\max }=0.002$ \\
\hline 4530 reflections & $\Delta\rangle_{\max }=0.66 \mathrm{e} \AA^{-3}$ \\
\hline 212 parameters & $\Delta\rangle_{\min }=-0.68 \mathrm{e} \AA^{-3}$ \\
\hline
\end{tabular}

\section{Special details}

Experimental. Collected, solved and refined by Hongyu Zhong

Geometry. All esds (except the esd in the dihedral angle between two 1.s. planes) are estimated using the full covariance matrix. The cell esds are taken into account individually in the estimation of esds in distances, angles and torsion angles; correlations between esds in cell parameters are only used when they are defined by crystal symmetry. An approximate (isotropic) treatment of cell esds is used for estimating esds involving 1.s. planes.

Fractional atomic coordinates and isotropic or equivalent isotropic displacement parameters $\left(\AA^{2}\right)$ for (SMK11)

\begin{tabular}{|l|l|l|l|l|}
\hline & $x$ & $y$ & $z$ & $U_{\text {iso }} * / U_{\text {eq }}$ \\
\hline C1 & $0.6653(2)$ & $0.0856(4)$ & $0.70461(18)$ & $0.0176(6)$ \\
\hline C2 & $0.6817(2)$ & $0.0733(4)$ & $0.63168(19)$ & $0.0190(6)$ \\
\hline C3 & $0.5991(2)$ & $0.1722(4)$ & $0.57194(18)$ & $0.0190(6)$ \\
\hline
\end{tabular}




\begin{tabular}{|c|c|c|c|c|}
\hline $\mathrm{C} 4$ & $0.5320(2)$ & $0.2425(4)$ & $0.60911(18)$ & $0.0184(6)$ \\
\hline $\mathrm{C} 5$ & $0.5739(2)$ & 0.1947 (4) & $0.69222(18)$ & $0.0174(6)$ \\
\hline C6 & $0.7639(3)$ & $-0.0364(5)$ & $0.6165(2)$ & $0.0295(7)$ \\
\hline H6A & 0.758364 & -0.021482 & 0.562261 & $0.044 *$ \\
\hline H6B & 0.833364 & -0.001656 & 0.651737 & $0.044 *$ \\
\hline $\mathrm{H} 6 \mathrm{C}$ & 0.752484 & -0.156184 & 0.625658 & $0.044 *$ \\
\hline C7 & $0.5805(3)$ & $0.1776(4)$ & $0.48442(19)$ & $0.0265(7)$ \\
\hline H7A & 0.638070 & 0.120544 & 0.475276 & $0.040^{*}$ \\
\hline H7B & 0.515021 & 0.119601 & 0.454948 & $0.040^{*}$ \\
\hline $\mathrm{H} 7 \mathrm{C}$ & 0.576522 & 0.296266 & 0.467245 & $0.040^{*}$ \\
\hline $\mathrm{C} 8$ & $0.4291(2)$ & $0.3369(4)$ & $0.5690(2)$ & $0.0241(6)$ \\
\hline H8A & 0.400094 & 0.370461 & 0.608158 & $0.036^{*}$ \\
\hline H8B & 0.441528 & 0.438542 & 0.542927 & $0.036^{*}$ \\
\hline $\mathrm{H} 8 \mathrm{C}$ & 0.379824 & 0.262043 & 0.530416 & $0.036^{*}$ \\
\hline C9 & $0.5206(2)$ & $0.2265(4)$ & $0.7508(2)$ & $0.0232(6)$ \\
\hline H9A & 0.461713 & 0.304101 & 0.727736 & $0.035^{*}$ \\
\hline H9B & 0.495461 & 0.118431 & 0.763712 & $0.035^{*}$ \\
\hline $\mathrm{H} 9 \mathrm{C}$ & 0.570556 & 0.277473 & 0.798350 & $0.035^{*}$ \\
\hline $\mathrm{C} 10$ & $0.7295(2)$ & $-0.0043(4)$ & $0.7810(2)$ & $0.0250(7)$ \\
\hline H10A & 0.702097 & 0.025686 & 0.821490 & $0.037 *$ \\
\hline H10B & 0.724998 & -0.127635 & 0.772913 & $0.037 *$ \\
\hline H10C & 0.802324 & 0.031740 & 0.797509 & $0.037 *$ \\
\hline C11 & $0.7873(2)$ & $0.4644(3)$ & $0.77204(15)$ & $0.0137(5)$ \\
\hline $\mathrm{C} 12$ & $0.7702(3)$ & $0.4631(4)$ & $0.84472(18)$ & $0.0228(6)$ \\
\hline H12 & 0.710843 & 0.407428 & 0.847761 & $0.027 *$ \\
\hline C13 & $0.8417(3)$ & $0.5447(6)$ & $0.9117(2)$ & $0.0360(9)$ \\
\hline H13 & 0.826496 & 0.551044 & 0.957940 & $0.043 *$ \\
\hline $\mathrm{C} 14$ & $0.9357(3)$ & $0.6169(5)$ & $0.9108(2)$ & $0.0307(8)$ \\
\hline H14 & 0.987386 & 0.656563 & 0.957781 & $0.037^{*}$ \\
\hline C15 & $0.9515(2)$ & $0.6293(4)$ & $0.83873(18)$ & $0.0221(6)$ \\
\hline H15 & 1.009416 & 0.689386 & 0.835583 & $0.026^{*}$ \\
\hline C16 & $0.8773(2)$ & $0.5482(4)$ & $0.77054(16)$ & $0.0146(5)$ \\
\hline C17 & $0.8891(2)$ & $0.5455(3)$ & $0.69354(16)$ & $0.0140(5)$ \\
\hline $\mathrm{C} 18$ & $0.9763(2)$ & $0.6331(4)$ & $0.67936(18)$ & $0.0188(6)$ \\
\hline H18A & 1.042724 & 0.593706 & 0.719145 & $0.023^{*}$ \\
\hline H18B & 0.970654 & 0.756661 & 0.687359 & $0.023 *$ \\
\hline C19 & $0.9816(3)$ & $0.6070(5)$ & $0.6006(2)$ & $0.0326(8)$ \\
\hline
\end{tabular}




\begin{tabular}{|l|l|l|l|l|}
\hline H19A & 1.008834 & 0.712430 & 0.585620 & $0.039^{*}$ \\
\hline H19B & 1.032461 & 0.515267 & 0.604562 & $0.039^{*}$ \\
\hline C20 & $0.8850(3)$ & $0.5639(6)$ & $0.5391(2)$ & $0.0361(9)$ \\
\hline H20A & 0.901720 & 0.511231 & 0.496248 & $0.043^{*}$ \\
\hline H20B & 0.847390 & 0.671247 & 0.518492 & $0.043^{*}$ \\
\hline C21 & $0.8130(2)$ & $0.4496(4)$ & $0.55778(17)$ & $0.0195(6)$ \\
\hline H21A & 0.741644 & 0.473049 & 0.520488 & $0.023^{*}$ \\
\hline H21B & 0.830222 & 0.331239 & 0.548670 & $0.023^{*}$ \\
\hline N1 & $0.81201(19)$ & $0.4598(3)$ & $0.63706(14)$ & $0.0168(5)$ \\
\hline Rh1 & $0.69275(2)$ & $0.35702(3)$ & $0.66815(2)$ & $0.01223(7)$ \\
\hline H1 & $0.647(3)$ & $0.540(6)$ & $0.654(3)$ & $0.045(12)^{*}$ \\
\hline
\end{tabular}

\section{Atomic displacement parameters $\left(\AA^{2}\right)$ for (SMK11)}

\begin{tabular}{|l|l|l|l|l|l|l|}
\hline & $U^{11}$ & $U^{22}$ & $U^{33}$ & $U^{12}$ & $U^{13}$ & $U^{23}$ \\
\hline C1 & $0.0124(12)$ & $0.0129(12)$ & $0.0277(15)$ & $-0.0026(10)$ & $0.0076(11)$ & $0.0010(11)$ \\
\hline C2 & $0.0159(13)$ & $0.0132(13)$ & $0.0282(15)$ & $-0.0027(11)$ & $0.0085(11)$ & $-0.0050(12)$ \\
\hline C3 & $0.0177(13)$ & $0.0142(13)$ & $0.0246(15)$ & $-0.0043(10)$ & $0.0070(11)$ & $-0.0047(11)$ \\
\hline C4 & $0.0151(13)$ & $0.0133(13)$ & $0.0242(14)$ & $-0.0043(10)$ & $0.0041(11)$ & $-0.0010(11)$ \\
\hline C5 & $0.0140(12)$ & $0.0148(13)$ & $0.0241(14)$ & $-0.0016(10)$ & $0.0077(11)$ & $-0.0001(11)$ \\
\hline C6 & $0.0258(16)$ & $0.0257(17)$ & $0.0399(19)$ & $0.0007(13)$ & $0.0154(14)$ & $-0.0111(14)$ \\
\hline C7 & $0.0325(17)$ & $0.0216(16)$ & $0.0240(15)$ & $-0.0088(13)$ & $0.0087(13)$ & $-0.0069(12)$ \\
\hline C8 & $0.0166(14)$ & $0.0196(15)$ & $0.0305(16)$ & $0.0003(11)$ & $0.0021(12)$ & $0.0008(12)$ \\
\hline C9 & $0.0186(14)$ & $0.0210(15)$ & $0.0343(17)$ & $-0.0005(12)$ & $0.0150(13)$ & $0.0014(13)$ \\
\hline C10 & $0.0175(14)$ & $0.0217(15)$ & $0.0353(17)$ & $0.0012(12)$ & $0.0091(13)$ & $0.0098(13)$ \\
\hline C11 & $0.0168(12)$ & $0.0131(12)$ & $0.0116(12)$ & $0.0027(10)$ & $0.0054(10)$ & $-0.0008(10)$ \\
\hline C12 & $0.0259(15)$ & $0.0277(16)$ & $0.0189(14)$ & $-0.0025(13)$ & $0.0129(12)$ & $-0.0008(12)$ \\
\hline C13 & $0.0354(19)$ & $0.059(3)$ & $0.0167(15)$ & $-0.0075(18)$ & $0.0131(14)$ & $-0.0098(16)$ \\
\hline C14 & $0.0326(18)$ & $0.039(2)$ & $0.0180(15)$ & $-0.0057(15)$ & $0.0056(13)$ & $-0.0097(14)$ \\
\hline C15 & $0.0176(14)$ & $0.0256(16)$ & $0.0209(14)$ & $0.0003(12)$ & $0.0046(11)$ & $-0.0053(12)$ \\
\hline C16 & $0.0141(12)$ & $0.0139(13)$ & $0.0155(12)$ & $0.0019(10)$ & $0.0052(10)$ & $-0.0007(10)$ \\
\hline C17 & $0.0132(12)$ & $0.0122(12)$ & $0.0174(13)$ & $0.0027(10)$ & $0.0066(10)$ & $0.0014(10)$ \\
\hline C18 & $0.0165(13)$ & $0.0163(13)$ & $0.0249(14)$ & $0.0020(11)$ & $0.0092(11)$ & $0.0035(11)$ \\
\hline C19 & $0.0269(17)$ & $0.044(2)$ & $0.0289(17)$ & $-0.0106(15)$ & $0.0127(14)$ & $0.0105(15)$ \\
\hline C20 & $0.0343(19)$ & $0.056(2)$ & $0.0248(17)$ & $-0.0131(18)$ & $0.0191(15)$ & $-0.0054(16)$ \\
\hline C21 & $0.0162(13)$ & $0.0263(16)$ & $0.0172(13)$ & $0.0032(11)$ & $0.0074(11)$ & $0.0035(11)$ \\
\hline N1 & $0.0192(11)$ & $0.0158(12)$ & $0.0177(11)$ & $0.0011(9)$ & $0.0095(9)$ & $0.0012(9)$ \\
\hline Rh1 & $0.01177(11)$ & $0.01150(11)$ & $0.01436(11)$ & $-0.00144(8)$ & $0.00588(8)$ & $-0.00115(8)$ \\
\hline
\end{tabular}


Geometric parameters $\left(A,{ }^{\circ}\right)$ for (SMK11)

\begin{tabular}{|c|c|c|c|}
\hline $\mathrm{C} 1-\mathrm{C} 2$ & $1.415(4)$ & $\mathrm{C} 10-\mathrm{H} 10 \mathrm{C}$ & 0.9600 \\
\hline $\mathrm{C} 1-\mathrm{C} 5$ & $1.443(4)$ & $\mathrm{C} 11-\mathrm{C} 16$ & $1.387(4)$ \\
\hline $\mathrm{C} 1-\mathrm{C} 10$ & $1.502(4)$ & $\mathrm{C} 11-\mathrm{C} 12$ & $1.412(4)$ \\
\hline $\mathrm{C} 1-\mathrm{Rh} 1$ & $2.263(3)$ & C11-Rh1 & $2.019(3)$ \\
\hline $\mathrm{C} 2-\mathrm{C} 3$ & $1.452(4)$ & $\mathrm{C} 12-\mathrm{C} 13$ & $1.391(5)$ \\
\hline $\mathrm{C} 2-\mathrm{C} 6$ & $1.499(4)$ & $\mathrm{C} 12-\mathrm{H} 12$ & 0.9300 \\
\hline $\mathrm{C} 2-\mathrm{Rh} 1$ & $2.271(3)$ & $\mathrm{C} 13-\mathrm{C} 14$ & $1.394(5)$ \\
\hline $\mathrm{C} 3-\mathrm{C} 4$ & $1.419(4)$ & $\mathrm{C} 13-\mathrm{H} 13$ & 0.9300 \\
\hline $\mathrm{C} 3-\mathrm{C} 7$ & $1.506(4)$ & $\mathrm{C} 14-\mathrm{C} 15$ & $1.397(5)$ \\
\hline $\mathrm{C} 3-\mathrm{Rh} 1$ & $2.243(3)$ & $\mathrm{C} 14-\mathrm{H} 14$ & 0.9300 \\
\hline $\mathrm{C} 4-\mathrm{C} 5$ & $1.443(4)$ & $\mathrm{C} 15-\mathrm{C} 16$ & $1.416(4)$ \\
\hline $\mathrm{C} 4-\mathrm{C} 8$ & $1.500(4)$ & $\mathrm{C} 15-\mathrm{H} 15$ & 0.9300 \\
\hline $\mathrm{C} 4-\mathrm{Rh} 1$ & $2.224(3)$ & $\mathrm{C} 16-\mathrm{C} 17$ & $1.455(4)$ \\
\hline $\mathrm{C} 5-\mathrm{C} 9$ & $1.502(4)$ & $\mathrm{C} 17-\mathrm{N} 1$ & $1.335(4)$ \\
\hline $\mathrm{C} 5-\mathrm{Rh} 1$ & $2.202(3)$ & $\mathrm{C} 17-\mathrm{C} 18$ & $1.460(4)$ \\
\hline C6-H6A & 0.9600 & $\mathrm{C} 18-\mathrm{C} 19$ & $1.463(5)$ \\
\hline C6-H6B & 0.9600 & C18-H18A & 0.9700 \\
\hline C6-H6C & 0.9600 & C18-H18B & 0.9700 \\
\hline C7-H7A & 0.9600 & $\mathrm{C} 19-\mathrm{C} 20$ & $1.410(5)$ \\
\hline C7-H7B & 0.9600 & C19-H19A & 0.9700 \\
\hline C7-H7C & 0.9600 & C19-H19B & 0.9700 \\
\hline $\mathrm{C} 8-\mathrm{H} 8 \mathrm{~A}$ & 0.9600 & $\mathrm{C} 20-\mathrm{C} 21$ & $1.441(5)$ \\
\hline $\mathrm{C} 8-\mathrm{H} 8 \mathrm{~B}$ & 0.9600 & $\mathrm{C} 20-\mathrm{H} 20 \mathrm{~A}$ & 0.9700 \\
\hline $\mathrm{C} 8-\mathrm{H} 8 \mathrm{C}$ & 0.9600 & $\mathrm{C} 20-\mathrm{H} 20 \mathrm{~B}$ & 0.9700 \\
\hline C9-H9A & 0.9600 & $\mathrm{C} 21-\mathrm{N} 1$ & $1.437(4)$ \\
\hline C9-H9B & 0.9600 & $\mathrm{C} 21-\mathrm{H} 21 \mathrm{~A}$ & 0.9700 \\
\hline C9-H9C & 0.9600 & $\mathrm{C} 21-\mathrm{H} 21 \mathrm{~B}$ & 0.9700 \\
\hline $\mathrm{C} 10-\mathrm{H} 10 \mathrm{~A}$ & 0.9600 & $\mathrm{~N} 1-\mathrm{Rh} 1$ & $2.051(2)$ \\
\hline $\mathrm{C} 10-\mathrm{H} 10 \mathrm{~B}$ & 0.9600 & $\mathrm{Rh} 1-\mathrm{H} 1$ & $1.53(5)$ \\
\hline $\mathrm{C} 2-\mathrm{C} 1-\mathrm{C} 5$ & $108.2(3)$ & $\mathrm{C} 14-\mathrm{C} 13-\mathrm{H} 13$ & 119.4 \\
\hline $\mathrm{C} 2-\mathrm{C} 1-\mathrm{C} 10$ & $126.0(3)$ & $\mathrm{C} 13-\mathrm{C} 14-\mathrm{C} 15$ & $119.3(3)$ \\
\hline $\mathrm{C} 5-\mathrm{C} 1-\mathrm{C} 10$ & $125.8(3)$ & $\mathrm{C} 13-\mathrm{C} 14-\mathrm{H} 14$ & 120.3 \\
\hline $\mathrm{C} 2-\mathrm{C} 1-\mathrm{Rh} 1$ & $72.14(17)$ & $\mathrm{C} 15-\mathrm{C} 14-\mathrm{H} 14$ & 120.3 \\
\hline $\mathrm{C} 5-\mathrm{C} 1-\mathrm{Rh} 1$ & $68.86(16)$ & $\mathrm{C} 14-\mathrm{C} 15-\mathrm{C} 16$ & $118.6(3)$ \\
\hline
\end{tabular}




\begin{tabular}{|c|c|c|c|}
\hline $\mathrm{C} 10-\mathrm{C} 1-\mathrm{Rh} 1$ & $126.3(2)$ & $\mathrm{C} 14-\mathrm{C} 15-\mathrm{H} 15$ & 120.7 \\
\hline $\mathrm{C} 1-\mathrm{C} 2-\mathrm{C} 3$ & $108.3(3)$ & $\mathrm{C} 16-\mathrm{C} 15-\mathrm{H} 15$ & 120.7 \\
\hline $\mathrm{C} 1-\mathrm{C} 2-\mathrm{C} 6$ & $125.9(3)$ & $\mathrm{C} 11-\mathrm{C} 16-\mathrm{C} 15$ & $122.4(3)$ \\
\hline $\mathrm{C} 3-\mathrm{C} 2-\mathrm{C} 6$ & $125.5(3)$ & $\mathrm{C} 11-\mathrm{C} 16-\mathrm{C} 17$ & $114.6(2)$ \\
\hline $\mathrm{C} 1-\mathrm{C} 2-\mathrm{Rh} 1$ & $71.48(16)$ & $\mathrm{C} 15-\mathrm{C} 16-\mathrm{C} 17$ & $123.0(3)$ \\
\hline $\mathrm{C} 3-\mathrm{C} 2-\mathrm{Rh} 1$ & $70.20(16)$ & $\mathrm{N} 1-\mathrm{C} 17-\mathrm{C} 16$ & $114.0(2)$ \\
\hline $\mathrm{C} 6-\mathrm{C} 2-\mathrm{Rh} 1$ & $128.4(2)$ & $\mathrm{N} 1-\mathrm{C} 17-\mathrm{C} 18$ & $123.4(3)$ \\
\hline $\mathrm{C} 4-\mathrm{C} 3-\mathrm{C} 2$ & $107.5(3)$ & $\mathrm{C} 16-\mathrm{C} 17-\mathrm{C} 18$ & $122.6(2)$ \\
\hline $\mathrm{C} 4-\mathrm{C} 3-\mathrm{C} 7$ & $126.5(3)$ & $\mathrm{C} 17-\mathrm{C} 18-\mathrm{C} 19$ & $115.9(3)$ \\
\hline $\mathrm{C} 2-\mathrm{C} 3-\mathrm{C} 7$ & $125.5(3)$ & $\mathrm{C} 17-\mathrm{C} 18-\mathrm{H} 18 \mathrm{~A}$ & 108.3 \\
\hline $\mathrm{C} 4-\mathrm{C} 3-\mathrm{Rh} 1$ & $70.73(16)$ & $\mathrm{C} 19-\mathrm{C} 18-\mathrm{H} 18 \mathrm{~A}$ & 108.3 \\
\hline $\mathrm{C} 2-\mathrm{C} 3-\mathrm{Rh} 1$ & $72.29(16)$ & $\mathrm{C} 17-\mathrm{C} 18-\mathrm{H} 18 \mathrm{~B}$ & 108.3 \\
\hline $\mathrm{C} 7-\mathrm{C} 3-\mathrm{Rh} 1$ & $128.6(2)$ & $\mathrm{C} 19-\mathrm{C} 18-\mathrm{H} 18 \mathrm{~B}$ & 108.3 \\
\hline $\mathrm{C} 3-\mathrm{C} 4-\mathrm{C} 5$ & $108.5(3)$ & $\mathrm{H} 18 \mathrm{~A}-\mathrm{C} 18-\mathrm{H} 18 \mathrm{~B}$ & 107.4 \\
\hline $\mathrm{C} 3-\mathrm{C} 4-\mathrm{C} 8$ & $126.8(3)$ & $\mathrm{C} 20-\mathrm{C} 19-\mathrm{C} 18$ & $115.8(3)$ \\
\hline $\mathrm{C} 5-\mathrm{C} 4-\mathrm{C} 8$ & $124.5(3)$ & $\mathrm{C} 20-\mathrm{C} 19-\mathrm{H} 19 \mathrm{~A}$ & 108.3 \\
\hline $\mathrm{C} 3-\mathrm{C} 4-\mathrm{Rh} 1$ & $72.23(16)$ & $\mathrm{C} 18-\mathrm{C} 19-\mathrm{H} 19 \mathrm{~A}$ & 108.3 \\
\hline $\mathrm{C} 5-\mathrm{C} 4-\mathrm{Rh} 1$ & $70.14(16)$ & $\mathrm{C} 20-\mathrm{C} 19-\mathrm{H} 19 \mathrm{~B}$ & 108.3 \\
\hline $\mathrm{C} 8-\mathrm{C} 4-\mathrm{Rh} 1$ & $127.6(2)$ & $\mathrm{C} 18-\mathrm{C} 19-\mathrm{H} 19 \mathrm{~B}$ & 108.3 \\
\hline $\mathrm{C} 1-\mathrm{C} 5-\mathrm{C} 4$ & $107.3(3)$ & H19A-C19-H19B & 107.4 \\
\hline $\mathrm{C} 1-\mathrm{C} 5-\mathrm{C} 9$ & $126.3(3)$ & $\mathrm{C} 19-\mathrm{C} 20-\mathrm{C} 21$ & $118.0(3)$ \\
\hline $\mathrm{C} 4-\mathrm{C} 5-\mathrm{C} 9$ & $125.6(3)$ & $\mathrm{C} 19-\mathrm{C} 20-\mathrm{H} 20 \mathrm{~A}$ & 107.8 \\
\hline $\mathrm{C} 1-\mathrm{C} 5-\mathrm{Rh} 1$ & $73.46(16)$ & $\mathrm{C} 21-\mathrm{C} 20-\mathrm{H} 20 \mathrm{~A}$ & 107.8 \\
\hline $\mathrm{C} 4-\mathrm{C} 5-\mathrm{Rh} 1$ & $71.80(16)$ & $\mathrm{C} 19-\mathrm{C} 20-\mathrm{H} 20 \mathrm{~B}$ & 107.8 \\
\hline $\mathrm{C} 9-\mathrm{C} 5-\mathrm{Rh} 1$ & $128.3(2)$ & $\mathrm{C} 21-\mathrm{C} 20-\mathrm{H} 20 \mathrm{~B}$ & 107.8 \\
\hline $\mathrm{C} 2-\mathrm{C} 6-\mathrm{H} 6 \mathrm{~A}$ & 109.5 & $\mathrm{H} 20 \mathrm{~A}-\mathrm{C} 20-\mathrm{H} 20 \mathrm{~B}$ & 107.1 \\
\hline $\mathrm{C} 2-\mathrm{C} 6-\mathrm{H} 6 \mathrm{~B}$ & 109.5 & $\mathrm{~N} 1-\mathrm{C} 21-\mathrm{C} 20$ & $117.0(3)$ \\
\hline $\mathrm{H} 6 \mathrm{~A}-\mathrm{C} 6-\mathrm{H} 6 \mathrm{~B}$ & 109.5 & $\mathrm{~N} 1-\mathrm{C} 21-\mathrm{H} 21 \mathrm{~A}$ & 108.1 \\
\hline $\mathrm{C} 2-\mathrm{C} 6-\mathrm{H} 6 \mathrm{C}$ & 109.5 & $\mathrm{C} 20-\mathrm{C} 21-\mathrm{H} 21 \mathrm{~A}$ & 108.1 \\
\hline $\mathrm{H} 6 \mathrm{~A}-\mathrm{C} 6-\mathrm{H} 6 \mathrm{C}$ & 109.5 & $\mathrm{~N} 1-\mathrm{C} 21-\mathrm{H} 21 \mathrm{~B}$ & 108.1 \\
\hline $\mathrm{H} 6 \mathrm{~B}-\mathrm{C} 6-\mathrm{H} 6 \mathrm{C}$ & 109.5 & $\mathrm{C} 20-\mathrm{C} 21-\mathrm{H} 21 \mathrm{~B}$ & 108.1 \\
\hline $\mathrm{C} 3-\mathrm{C} 7-\mathrm{H} 7 \mathrm{~A}$ & 109.5 & $\mathrm{H} 21 \mathrm{~A}-\mathrm{C} 21-\mathrm{H} 21 \mathrm{~B}$ & 107.3 \\
\hline $\mathrm{C} 3-\mathrm{C} 7-\mathrm{H} 7 \mathrm{~B}$ & 109.5 & $\mathrm{C} 17-\mathrm{N} 1-\mathrm{C} 21$ & $119.8(2)$ \\
\hline $\mathrm{H} 7 \mathrm{~A}-\mathrm{C} 7-\mathrm{H} 7 \mathrm{~B}$ & 109.5 & C17-N1-Rh1 & $117.06(18)$ \\
\hline $\mathrm{C} 3-\mathrm{C} 7-\mathrm{H} 7 \mathrm{C}$ & 109.5 & $\mathrm{C} 21-\mathrm{N} 1-\mathrm{Rh} 1$ & $123.12(19)$ \\
\hline $\mathrm{H} 7 \mathrm{~A}-\mathrm{C} 7-\mathrm{H} 7 \mathrm{C}$ & 109.5 & $\mathrm{C} 11-\mathrm{Rh} 1-\mathrm{N} 1$ & $78.55(10)$ \\
\hline $\mathrm{H} 7 \mathrm{~B}-\mathrm{C} 7-\mathrm{H} 7 \mathrm{C}$ & 109.5 & $\mathrm{C} 11-\mathrm{Rh} 1-\mathrm{C} 5$ & $108.04(11)$ \\
\hline
\end{tabular}




\begin{tabular}{|c|c|c|c|}
\hline $\mathrm{C} 4-\mathrm{C} 8-\mathrm{H} 8 \mathrm{~A}$ & 109.5 & $\mathrm{~N} 1-\mathrm{Rh} 1-\mathrm{C} 5$ & $167.78(10)$ \\
\hline $\mathrm{C} 4-\mathrm{C} 8-\mathrm{H} 8 \mathrm{~B}$ & 109.5 & $\mathrm{C} 11-\mathrm{Rh} 1-\mathrm{C} 4$ & $141.53(11)$ \\
\hline $\mathrm{H} 8 \mathrm{~A}-\mathrm{C} 8-\mathrm{H} 8 \mathrm{~B}$ & 109.5 & $\mathrm{~N} 1-\mathrm{Rh} 1-\mathrm{C} 4$ & $138.71(10)$ \\
\hline $\mathrm{C} 4-\mathrm{C} 8-\mathrm{H} 8 \mathrm{C}$ & 109.5 & $\mathrm{C} 5-\mathrm{Rh} 1-\mathrm{C} 4$ & $38.06(11)$ \\
\hline $\mathrm{H} 8 \mathrm{~A}-\mathrm{C} 8-\mathrm{H} 8 \mathrm{C}$ & 109.5 & $\mathrm{C} 11-\mathrm{Rh} 1-\mathrm{C} 3$ & $164.64(11)$ \\
\hline $\mathrm{H} 8 \mathrm{~B}-\mathrm{C} 8-\mathrm{H} 8 \mathrm{C}$ & 109.5 & $\mathrm{~N} 1-\mathrm{Rh} 1-\mathrm{C} 3$ & $108.13(10)$ \\
\hline $\mathrm{C} 5-\mathrm{C} 9-\mathrm{H} 9 \mathrm{~A}$ & 109.5 & $\mathrm{C} 5-\mathrm{Rh} 1-\mathrm{C} 3$ & $63.00(11)$ \\
\hline $\mathrm{C} 5-\mathrm{C} 9-\mathrm{H} 9 \mathrm{~B}$ & 109.5 & $\mathrm{C} 4-\mathrm{Rh} 1-\mathrm{C} 3$ & $37.04(11)$ \\
\hline $\mathrm{H} 9 \mathrm{~A}-\mathrm{C} 9-\mathrm{H} 9 \mathrm{~B}$ & 109.5 & $\mathrm{C} 11-\mathrm{Rh} 1-\mathrm{C} 1$ & $102.97(11)$ \\
\hline $\mathrm{C} 5-\mathrm{C} 9-\mathrm{H} 9 \mathrm{C}$ & 109.5 & $\mathrm{~N} 1-\mathrm{Rh} 1-\mathrm{C} 1$ & $131.70(10)$ \\
\hline $\mathrm{H} 9 \mathrm{~A}-\mathrm{C} 9-\mathrm{H} 9 \mathrm{C}$ & 109.5 & $\mathrm{C} 5-\mathrm{Rh} 1-\mathrm{C} 1$ & $37.68(10)$ \\
\hline $\mathrm{H} 9 \mathrm{~B}-\mathrm{C} 9-\mathrm{H} 9 \mathrm{C}$ & 109.5 & $\mathrm{C} 4-\mathrm{Rh} 1-\mathrm{C} 1$ & $62.42(10)$ \\
\hline $\mathrm{C} 1-\mathrm{C} 10-\mathrm{H} 10 \mathrm{~A}$ & 109.5 & $\mathrm{C} 3-\mathrm{Rh} 1-\mathrm{C} 1$ & $62.11(11)$ \\
\hline $\mathrm{C} 1-\mathrm{C} 10-\mathrm{H} 10 \mathrm{~B}$ & 109.5 & $\mathrm{C} 11-\mathrm{Rh} 1-\mathrm{C} 2$ & $127.97(11)$ \\
\hline $\mathrm{H} 10 \mathrm{~A}-\mathrm{C} 10-\mathrm{H} 10 \mathrm{~B}$ & 109.5 & $\mathrm{~N} 1-\mathrm{Rh} 1-\mathrm{C} 2$ & $105.48(10)$ \\
\hline $\mathrm{C} 1-\mathrm{C} 10-\mathrm{H} 10 \mathrm{C}$ & 109.5 & $\mathrm{C} 5-\mathrm{Rh} 1-\mathrm{C} 2$ & $62.31(11)$ \\
\hline $\mathrm{H} 10 \mathrm{~A}-\mathrm{C} 10-\mathrm{H} 10 \mathrm{C}$ & 109.5 & $\mathrm{C} 4-\mathrm{Rh} 1-\mathrm{C} 2$ & $62.01(11)$ \\
\hline $\mathrm{H} 10 \mathrm{~B}-\mathrm{C} 10-\mathrm{H} 10 \mathrm{C}$ & 109.5 & $\mathrm{C} 3-\mathrm{Rh} 1-\mathrm{C} 2$ & $37.51(11)$ \\
\hline $\mathrm{C} 16-\mathrm{C} 11-\mathrm{C} 12$ & $117.7(3)$ & $\mathrm{C} 1-\mathrm{Rh} 1-\mathrm{C} 2$ & $36.37(11)$ \\
\hline $\mathrm{C} 16-\mathrm{C} 11-\mathrm{Rh} 1$ & $115.76(19)$ & $\mathrm{C} 11-\mathrm{Rh} 1-\mathrm{H} 1$ & $81.6(16)$ \\
\hline $\mathrm{C} 12-\mathrm{C} 11-\mathrm{Rh} 1$ & $126.5(2)$ & $\mathrm{N} 1-\mathrm{Rh} 1-\mathrm{H} 1$ & $85.0(16)$ \\
\hline $\mathrm{C} 13-\mathrm{C} 12-\mathrm{C} 11$ & $120.4(3)$ & $\mathrm{C} 5-\mathrm{Rh} 1-\mathrm{H} 1$ & $105.9(16)$ \\
\hline $\mathrm{C} 13-\mathrm{C} 12-\mathrm{H} 12$ & 119.8 & $\mathrm{C} 4-\mathrm{Rh} 1-\mathrm{H} 1$ & $91.2(17)$ \\
\hline $\mathrm{C} 11-\mathrm{C} 12-\mathrm{H} 12$ & 119.8 & $\mathrm{C} 3-\mathrm{Rh} 1-\mathrm{H} 1$ & $112.4(17)$ \\
\hline $\mathrm{C} 12-\mathrm{C} 13-\mathrm{C} 14$ & $121.1(3)$ & $\mathrm{C} 1-\mathrm{Rh} 1-\mathrm{H} 1$ & $143.3(16)$ \\
\hline $\mathrm{C} 12-\mathrm{C} 13-\mathrm{H} 13$ & 119.4 & $\mathrm{C} 2-\mathrm{Rh} 1-\mathrm{H} 1$ & $149.7(17)$ \\
\hline $\mathrm{C} 5-\mathrm{C} 1-\mathrm{C} 2-\mathrm{C} 3$ & $-1.1(3)$ & $\mathrm{C} 8-\mathrm{C} 4-\mathrm{C} 5-\mathrm{C} 1$ & $172.0(3)$ \\
\hline $\mathrm{C} 10-\mathrm{C} 1-\mathrm{C} 2-\mathrm{C} 3$ & $176.9(3)$ & $\mathrm{Rh} 1-\mathrm{C} 4-\mathrm{C} 5-\mathrm{C} 1$ & $-65.37(19)$ \\
\hline $\mathrm{Rh} 1-\mathrm{C} 1-\mathrm{C} 2-\mathrm{C} 3$ & $-60.8(2)$ & $\mathrm{C} 3-\mathrm{C} 4-\mathrm{C} 5-\mathrm{C} 9$ & $-173.1(3)$ \\
\hline $\mathrm{C} 5-\mathrm{C} 1-\mathrm{C} 2-\mathrm{C} 6$ & $-175.9(3)$ & $\mathrm{C} 8-\mathrm{C} 4-\mathrm{C} 5-\mathrm{C} 9$ & $1.9(5)$ \\
\hline $\mathrm{C} 10-\mathrm{C} 1-\mathrm{C} 2-\mathrm{C} 6$ & $2.2(5)$ & $\mathrm{Rh} 1-\mathrm{C} 4-\mathrm{C} 5-\mathrm{C} 9$ & $124.5(3)$ \\
\hline $\mathrm{Rh} 1-\mathrm{C} 1-\mathrm{C} 2-\mathrm{C} 6$ & $124.4(3)$ & $\mathrm{C} 3-\mathrm{C} 4-\mathrm{C} 5-\mathrm{Rh} 1$ & $62.4(2)$ \\
\hline $\mathrm{C} 5-\mathrm{C} 1-\mathrm{C} 2-\mathrm{Rh} 1$ & $59.7(2)$ & $\mathrm{C} 8-\mathrm{C} 4-\mathrm{C} 5-\mathrm{Rh} 1$ & $-122.6(3)$ \\
\hline $\mathrm{C} 10-\mathrm{C} 1-\mathrm{C} 2-\mathrm{Rh} 1$ & $-122.3(3)$ & $\begin{array}{l}\mathrm{C} 16-\mathrm{C} 11-\mathrm{C} 12- \\
\mathrm{C} 13\end{array}$ & $0.4(5)$ \\
\hline $\mathrm{C} 1-\mathrm{C} 2-\mathrm{C} 3-\mathrm{C} 4$ & $-0.7(3)$ & $\begin{array}{l}\mathrm{Rh} 1-\mathrm{C} 11-\mathrm{C} 12- \\
\mathrm{C} 13\end{array}$ & $-179.2(3)$ \\
\hline
\end{tabular}




\begin{tabular}{|c|c|c|c|}
\hline $\mathrm{C} 6-\mathrm{C} 2-\mathrm{C} 3-\mathrm{C} 4$ & $174.1(3)$ & $\begin{array}{l}\mathrm{C} 11-\mathrm{C} 12-\mathrm{C} 13- \\
\mathrm{C} 14\end{array}$ & $-5.3(6)$ \\
\hline $\mathrm{Rh} 1-\mathrm{C} 2-\mathrm{C} 3-\mathrm{C} 4$ & $-62.3(2)$ & $\begin{array}{l}\mathrm{C} 12-\mathrm{C} 13-\mathrm{C} 14- \\
\mathrm{C} 15\end{array}$ & $9.0(6)$ \\
\hline $\mathrm{C} 1-\mathrm{C} 2-\mathrm{C} 3-\mathrm{C} 7$ & $-173.2(3)$ & $\begin{array}{l}\mathrm{C} 13-\mathrm{C} 14-\mathrm{C} 15- \\
\mathrm{C} 16\end{array}$ & $-7.8(5)$ \\
\hline $\mathrm{C} 6-\mathrm{C} 2-\mathrm{C} 3-\mathrm{C} 7$ & $1.6(5)$ & $\begin{array}{l}\mathrm{C} 12-\mathrm{C} 11-\mathrm{C} 16- \\
\mathrm{C} 15\end{array}$ & $0.6(4)$ \\
\hline $\mathrm{Rh} 1-\mathrm{C} 2-\mathrm{C} 3-\mathrm{C} 7$ & $125.2(3)$ & $\begin{array}{l}\mathrm{Rh} 1-\mathrm{C} 11-\mathrm{C} 16- \\
\mathrm{C} 15\end{array}$ & $-179.8(2)$ \\
\hline $\mathrm{C} 1-\mathrm{C} 2-\mathrm{C} 3-\mathrm{Rh} 1$ & $61.6(2)$ & $\begin{array}{l}\mathrm{C} 12-\mathrm{C} 11-\mathrm{C} 16- \\
\mathrm{C} 17\end{array}$ & $-179.5(3)$ \\
\hline $\mathrm{C} 6-\mathrm{C} 2-\mathrm{C} 3-\mathrm{Rh} 1$ & $-123.6(3)$ & $\begin{array}{l}\mathrm{Rh} 1-\mathrm{C} 11-\mathrm{C} 16- \\
\mathrm{C} 17\end{array}$ & $0.1(3)$ \\
\hline $\mathrm{C} 2-\mathrm{C} 3-\mathrm{C} 4-\mathrm{C} 5$ & $2.3(3)$ & $\begin{array}{l}\mathrm{C} 14-\mathrm{C} 15-\mathrm{C} 16- \\
\mathrm{C} 11\end{array}$ & $3.1(5)$ \\
\hline $\mathrm{C} 7-\mathrm{C} 3-\mathrm{C} 4-\mathrm{C} 5$ & $174.7(3)$ & $\begin{array}{l}\mathrm{C} 14-\mathrm{C} 15-\mathrm{C} 16- \\
\mathrm{C} 17\end{array}$ & $-176.8(3)$ \\
\hline $\mathrm{Rh} 1-\mathrm{C} 3-\mathrm{C} 4-\mathrm{C} 5$ & $-61.1(2)$ & $\mathrm{C} 11-\mathrm{C} 16-\mathrm{C} 17-\mathrm{N} 1$ & $-1.1(4)$ \\
\hline $\mathrm{C} 2-\mathrm{C} 3-\mathrm{C} 4-\mathrm{C} 8$ & $-172.6(3)$ & $\mathrm{C} 15-\mathrm{C} 16-\mathrm{C} 17-\mathrm{N} 1$ & $178.8(3)$ \\
\hline $\mathrm{C} 7-\mathrm{C} 3-\mathrm{C} 4-\mathrm{C} 8$ & $-0.1(5)$ & $\begin{array}{l}\mathrm{C} 11-\mathrm{C} 16-\mathrm{C} 17- \\
\mathrm{C} 18\end{array}$ & $177.3(3)$ \\
\hline $\mathrm{Rh} 1-\mathrm{C} 3-\mathrm{C} 4-\mathrm{C} 8$ & $124.1(3)$ & $\begin{array}{l}\mathrm{C} 15-\mathrm{C} 16-\mathrm{C} 17- \\
\mathrm{C} 18\end{array}$ & $-2.8(4)$ \\
\hline $\mathrm{C} 2-\mathrm{C} 3-\mathrm{C} 4-\mathrm{Rh} 1$ & $63.4(2)$ & $\mathrm{N} 1-\mathrm{C} 17-\mathrm{C} 18-\mathrm{C} 19$ & $-6.2(4)$ \\
\hline $\mathrm{C} 7-\mathrm{C} 3-\mathrm{C} 4-\mathrm{Rh} 1$ & $-124.2(3)$ & $\begin{array}{l}\mathrm{C} 16-\mathrm{C} 17-\mathrm{C} 18- \\
\mathrm{C} 19\end{array}$ & $175.5(3)$ \\
\hline $\mathrm{C} 2-\mathrm{C} 1-\mathrm{C} 5-\mathrm{C} 4$ & $2.5(3)$ & $\begin{array}{l}\mathrm{C} 17-\mathrm{C} 18-\mathrm{C} 19- \\
\mathrm{C} 20\end{array}$ & $24.5(5)$ \\
\hline $\mathrm{C} 10-\mathrm{C} 1-\mathrm{C} 5-\mathrm{C} 4$ & $-175.5(3)$ & $\begin{array}{l}\mathrm{C} 18-\mathrm{C} 19-\mathrm{C} 20- \\
\mathrm{C} 21\end{array}$ & $-38.0(5)$ \\
\hline $\mathrm{Rh} 1-\mathrm{C} 1-\mathrm{C} 5-\mathrm{C} 4$ & $64.27(19)$ & $\mathrm{C} 19-\mathrm{C} 20-\mathrm{C} 21-\mathrm{N} 1$ & $32.3(5)$ \\
\hline $\mathrm{C} 2-\mathrm{C} 1-\mathrm{C} 5-\mathrm{C} 9$ & $172.5(3)$ & $\mathrm{C} 16-\mathrm{C} 17-\mathrm{N} 1-\mathrm{C} 21$ & $178.9(2)$ \\
\hline $\mathrm{C} 10-\mathrm{C} 1-\mathrm{C} 5-\mathrm{C} 9$ & $-5.5(5)$ & $\mathrm{C} 18-\mathrm{C} 17-\mathrm{N} 1-\mathrm{C} 21$ & $0.5(4)$ \\
\hline $\mathrm{Rh} 1-\mathrm{C} 1-\mathrm{C} 5-\mathrm{C} 9$ & $-125.7(3)$ & $\mathrm{C} 16-\mathrm{C} 17-\mathrm{N} 1-\mathrm{Rh} 1$ & $1.6(3)$ \\
\hline $\mathrm{C} 2-\mathrm{C} 1-\mathrm{C} 5-\mathrm{Rh} 1$ & $-61.8(2)$ & $\mathrm{C} 18-\mathrm{C} 17-\mathrm{N} 1-\mathrm{Rh} 1$ & $-176.8(2)$ \\
\hline $\mathrm{C} 10-\mathrm{C} 1-\mathrm{C} 5-\mathrm{Rh} 1$ & $120.2(3)$ & $\mathrm{C} 20-\mathrm{C} 21-\mathrm{N} 1-\mathrm{C} 17$ & $-12.9(4)$ \\
\hline $\mathrm{C} 3-\mathrm{C} 4-\mathrm{C} 5-\mathrm{C} 1$ & $-2.9(3)$ & $\mathrm{C} 20-\mathrm{C} 21-\mathrm{N} 1-\mathrm{Rh} 1$ & $164.2(3)$ \\
\hline
\end{tabular}

Document origin: publCIF [Westrip, S. P. (2010). J. Apply. Cryst., 43, 920-925].

\section{Compound 5:}




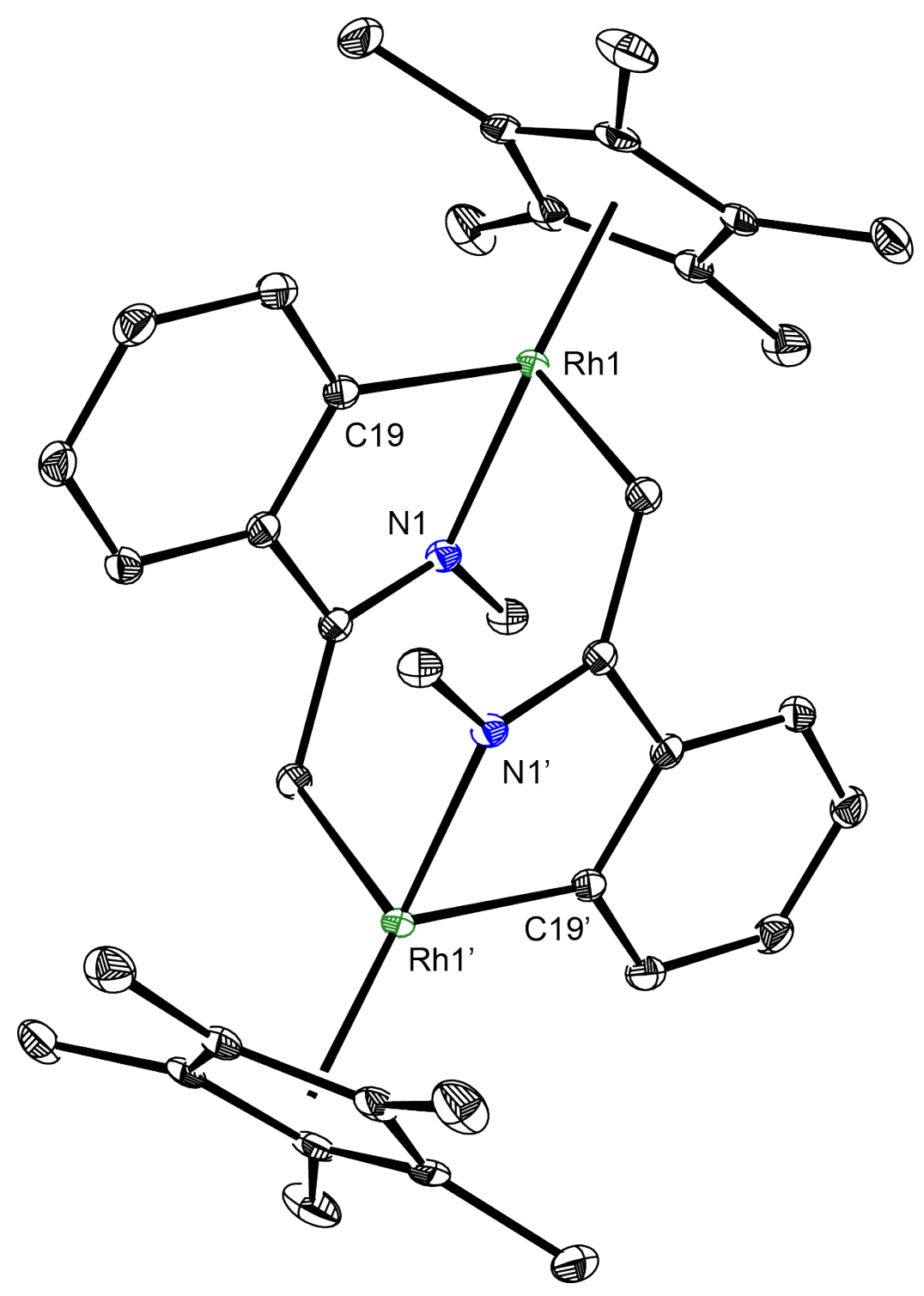

Table S4

Experimental details

\begin{tabular}{|l|l|}
\hline Crystal data & \multicolumn{2}{|l|}{} \\
\hline Chemical formula & $\mathrm{C}_{38} \mathrm{H}_{48} \mathrm{~N}_{2} \mathrm{Rh}_{2}$ \\
\hline$M_{\mathrm{r}}$ & 738.60 \\
\hline Crystal system, space group & Triclinic, $P^{-} 1$ \\
\hline Temperature (K) & 100 \\
\hline$a, b, c(\AA)$ & $8.6741(2), 9.5339(2), 10.0675(2)$ \\
\hline$\alpha, \beta, \gamma\left(^{\circ}\right)$ & $99.450(1), 109.730(1), 90.874(1)$ \\
\hline
\end{tabular}




\begin{tabular}{|c|c|}
\hline$V\left(\AA^{3}\right)$ & $770.81(3)$ \\
\hline$Z$ & 1 \\
\hline Radiation type & $\mathrm{Cu} K \alpha$ \\
\hline$\mu\left(\mathrm{mm}^{-1}\right)$ & 8.87 \\
\hline Crystal size $(\mathrm{mm})$ & $0.18 \times 0.14 \times 0.09$ \\
\hline \multicolumn{2}{|l|}{ Data collection } \\
\hline Diffractometer & Bruker $A P E X$-II CCD \\
\hline Absorption correction & $\begin{array}{l}\text { Multi-scan } \\
\text { TWINABS BRUKER AXS }\end{array}$ \\
\hline$T_{\min }, T_{\max }$ & $0.595,0.753$ \\
\hline $\begin{array}{l}\text { No. of measured, independent and } \\
\text { observed }[I>2 \sigma(I)] \text { reflections }\end{array}$ & $11027,2663,2632$ \\
\hline$R_{\text {int }}$ & 0.017 \\
\hline$(\sin \theta / \lambda)_{\max }\left(\AA^{-1}\right)$ & 0.595 \\
\hline \multicolumn{2}{|l|}{ Refinement } \\
\hline$R\left[F^{2}>2 \sigma\left(F^{2}\right)\right], w R\left(F^{2}\right), S$ & $0.019,0.051,1.07$ \\
\hline No. of reflections & 2663 \\
\hline No. of parameters & 195 \\
\hline H-atom treatment & H-atom parameters constrained \\
\hline$\left.\Delta\rangle_{\max }, \Delta\right\rangle_{\min }\left(\mathrm{e} \AA^{-3}\right)$ & $0.53,-0.48$ \\
\hline
\end{tabular}

Computer programs: Bruker APEX2, Bruker SAINT, SHELXT 2014/4 (Sheldrick, 2014), SHELXL2018/3 (Sheldrick, 2018).

Document origin: publCIF [Westrip, S. P. (2010). J. Apply. Cryst., 43, 920-925].

\section{full crystallographic data}

\section{Computing details}

Data collection: Bruker APEX2; cell refinement: Bruker SAINT; data reduction: Bruker SAINT; program(s) used to solve structure: SHELXT 2014/4 (Sheldrick, 2014); program(s) used to refine structure: SHELXL2018/3 (Sheldrick, 2018).

\section{(SMK20)}




\section{Crystal data}

\begin{tabular}{|l|l|}
\hline $\mathrm{C}_{38} \mathrm{H}_{48} \mathrm{~N}_{2} \mathrm{Rh}_{2}$ & $Z=1$ \\
\hline$M_{r}=738.60$ & $F(000)=380$ \\
\hline Triclinic, $P^{-} 1$ & $D_{\mathrm{x}}=1.591 \mathrm{Mg} \mathrm{m}^{-3}$ \\
\hline$a=8.6741(2) \AA$ & $\mathrm{Cu}$ Ka radiation, $\lambda=1.54178 \AA$ \\
\hline$b=9.5339(2) \AA$ & Cell parameters from 9966 reflections \\
\hline$c=10.0675(2) \AA$ & $\theta=4.7-66.6^{\circ}$ \\
\hline$\alpha=99.450(1)^{\circ}$ & $\mu=8.87 \mathrm{~mm}^{-1}$ \\
\hline$\beta=109.730(1)^{\circ}$ & $T=100 \mathrm{~K}$ \\
\hline$\gamma=90.874(1)^{\circ}$ & Plate, orange \\
\hline$V=770.81(3) \AA^{3}$ & $0.18 \times 0.14 \times 0.09 \mathrm{~mm}$ \\
\hline &
\end{tabular}

\section{Data collection}

\begin{tabular}{|l|l|}
\hline $\begin{array}{l}\text { Bruker APEX-II CCD } \\
\text { diffractometer }\end{array}$ & 2632 reflections with $I>2 \sigma(I)$ \\
\hline Radiation source: I $\mu$ S microfocus source & $R_{\text {int }}=0.017$ \\
\hline$\phi$ and $\omega$ scans & $\theta_{\max }=66.6^{\circ}, \theta_{\min }=4.7^{\circ}$ \\
\hline $\begin{array}{l}\text { Absorption correction: multi-scan } \\
\text { TWINABS BRUKER AXS }\end{array}$ & $h=-10 \rightarrow 10$ \\
\hline$T_{\min }=0.595, T_{\max }=0.753$ & $k=-11 \rightarrow 11$ \\
\hline 11027 measured reflections & $l=-11 \rightarrow 11$ \\
\hline 2663 independent reflections & \\
\hline
\end{tabular}

\section{Refinement}

\begin{tabular}{|l|l|}
\hline Refinement on $F^{2}$ & 0 restraints \\
\hline Least-squares matrix: full & $\begin{array}{l}\text { Hydrogen site location: inferred from } \\
\text { neighbouring sites }\end{array}$ \\
\hline$R\left[F^{2}>2 \sigma\left(F^{2}\right)\right]=0.019$ & H-atom parameters constrained \\
\hline$w R\left(F^{2}\right)=0.051$ & $\begin{array}{l}w=1 /\left[\sigma^{2}\left(F_{\mathrm{o}}{ }^{2}\right)+(0.0294 P)^{2}+0.7759 P\right] \\
\text { where } P=\left(F_{\mathrm{o}}{ }^{2}+2 F_{\mathrm{c}}{ }^{2}\right) / 3\end{array}$ \\
\hline$S=1.07$ & $(\Delta / \sigma)_{\max }=0.002$ \\
\hline 2663 reflections & $\Delta\rangle_{\max }=0.53$ e $\AA^{-3}$ \\
\hline 195 parameters & $\Delta\rangle_{\min }=-0.48 \mathrm{e} \AA^{-3}$ \\
\hline
\end{tabular}

\section{Special details}

Experimental. Collected, solved and refined by Hongyu Zhong

Geometry. All esds (except the esd in the dihedral angle between two 1.s. planes) are estimated 
using the full covariance matrix. The cell esds are taken into account individually in the estimation of esds in distances, angles and torsion angles; correlations between esds in cell parameters are only used when they are defined by crystal symmetry. An approximate (isotropic) treatment of cell esds is used for estimating esds involving l.s. planes.

Fractional atomic coordinates and isotropic or equivalent isotropic displacement parameters $\left(\AA^{2}\right)$ for (SMK20)

\begin{tabular}{|c|c|c|c|c|}
\hline & $x$ & $y$ & $z$ & $U_{\text {iso }} * / U_{\text {eq }}$ \\
\hline $\mathrm{C} 1$ & $0.3592(3)$ & $0.3408(2)$ & $0.8173(2)$ & $0.0232(5)$ \\
\hline $\mathrm{C} 2$ & $0.5111(3)$ & $0.2956(2)$ & $0.9056(2)$ & $0.0204(4)$ \\
\hline C3 & $0.6406(3)$ & $0.3582(2)$ & $0.8766(2)$ & $0.0208(4)$ \\
\hline $\mathrm{C} 4$ & $0.5726(3)$ & $0.4474(2)$ & $0.7722(2)$ & $0.0237(5)$ \\
\hline C5 & 0.3989 (3) & $0.4406(2)$ & 0.7392 (2) & $0.0259(5)$ \\
\hline C6 & $0.1946(3)$ & $0.3073(3)$ & $0.8281(3)$ & $0.0366(6)$ \\
\hline H6A & 0.196394 & 0.345463 & 0.925205 & $0.055^{*}$ \\
\hline H6B & 0.169980 & 0.203707 & 0.807696 & $0.055^{*}$ \\
\hline $\mathrm{H} 6 \mathrm{C}$ & 0.109860 & 0.350849 & 0.758521 & $0.055^{*}$ \\
\hline C7 & $0.5242(3)$ & $0.2036(3)$ & $1.0146(2)$ & $0.0289(5)$ \\
\hline H7A & 0.628146 & 0.158017 & 1.034905 & $0.043^{*}$ \\
\hline H7B & 0.432390 & 0.130167 & 0.977587 & $0.043^{*}$ \\
\hline $\mathrm{H} 7 \mathrm{C}$ & 0.520702 & 0.262196 & 1.102999 & $0.043 *$ \\
\hline $\mathrm{C} 8$ & $0.8206(3)$ & $0.3438(3)$ & $0.9511(3)$ & $0.0296(5)$ \\
\hline H8A & 0.852560 & 0.388531 & 1.052357 & $0.044 *$ \\
\hline H8B & 0.885143 & 0.390913 & 0.905443 & $0.044^{*}$ \\
\hline $\mathrm{H} 8 \mathrm{C}$ & 0.841332 & 0.242534 & 0.944084 & $0.044 *$ \\
\hline C9 & $0.6711(4)$ & $0.5412(3)$ & 0.7199 (3) & $0.0366(6)$ \\
\hline H9A & 0.601516 & 0.564725 & 0.628337 & $0.055^{*}$ \\
\hline H9B & 0.764015 & 0.491050 & 0.706368 & $0.055^{*}$ \\
\hline H9C & 0.712244 & 0.629071 & 0.790602 & $0.055^{*}$ \\
\hline $\mathrm{C} 10$ & $0.2801(4)$ & $0.5282(3)$ & $0.6472(3)$ & $0.0395(7)$ \\
\hline $\mathrm{H} 10 \mathrm{~A}$ & 0.338163 & 0.585162 & 0.603679 & $0.059 *$ \\
\hline H10B & 0.231286 & 0.591702 & 0.706414 & $0.059^{*}$ \\
\hline $\mathrm{H} 10 \mathrm{C}$ & 0.193187 & 0.464856 & 0.571539 & $0.059^{*}$ \\
\hline C11 & 0.7777 (3) & $0.1774(2)$ & $0.5883(2)$ & $0.0205(4)$ \\
\hline H11A & 0.816648 & 0.230510 & 0.686929 & $0.031 *$ \\
\hline H11B & 0.821840 & 0.083358 & 0.586396 & $0.031 *$ \\
\hline $\mathrm{H} 11 \mathrm{C}$ & 0.814805 & 0.229943 & 0.527352 & $0.031^{*}$ \\
\hline $\mathrm{C} 12$ & 0.5818 & $-0.0047(2)$ & $0.3123(2)$ & 0.0185 (4) \\
\hline
\end{tabular}




\begin{tabular}{|l|l|l|l|l|}
\hline H12A & 0.701565 & 0.018025 & 0.340468 & $0.022^{*}$ \\
\hline H12B & 0.529288 & 0.007560 & 0.211476 & $0.022^{*}$ \\
\hline C13 & $0.5163(3)$ & $0.0923(2)$ & $0.4050(2)$ & $0.0167(4)$ \\
\hline C14 & $0.3386(3)$ & $0.1104(2)$ & $0.3635(2)$ & $0.0179(4)$ \\
\hline C15 & $0.2268(3)$ & $0.0709(2)$ & $0.2238(2)$ & $0.0210(4)$ \\
\hline H15 & 0.263678 & 0.028161 & 0.148972 & $0.025^{*}$ \\
\hline C16 & $0.0620(3)$ & $0.0942(2)$ & $0.1944(2)$ & $0.0245(5)$ \\
\hline H16 & -0.014078 & 0.068311 & 0.099439 & $0.029^{*}$ \\
\hline C17 & $0.0092(3)$ & $0.1555(2)$ & $0.3046(3)$ & $0.0242(5)$ \\
\hline H17 & -0.103682 & 0.170996 & 0.284830 & $0.029^{*}$ \\
\hline C18 & $0.1195(3)$ & $0.1946(2)$ & $0.4435(2)$ & $0.0217(4)$ \\
\hline H18 & 0.080608 & 0.235351 & 0.517791 & $0.026^{*}$ \\
\hline C19 & $0.2874(3)$ & $0.1750(2)$ & $0.4765(2)$ & $0.0173(4)$ \\
\hline N1 & $0.5981(2)$ & $0.15992(18)$ & $0.53470(19)$ & $0.0167(4)$ \\
\hline Rh1 & $0.46545(2)$ & $0.22419(2)$ & $0.66888(2)$ & $0.01472(7)$ \\
\hline
\end{tabular}

\section{Atomic displacement parameters $\left(\AA^{2}\right)$ for (SMK20)}

\begin{tabular}{|l|l|l|l|l|l|l|}
\hline & $U^{11}$ & $U^{22}$ & $U^{33}$ & $U^{12}$ & $U^{13}$ & $U^{23}$ \\
\hline C1 & $0.0244(11)$ & $0.0226(11)$ & $0.0184(11)$ & $0.0026(9)$ & $0.0081(9)$ & $-0.0095(9)$ \\
\hline C2 & $0.0288(11)$ & $0.0149(10)$ & $0.0136(10)$ & $0.0010(8)$ & $0.0064(9)$ & $-0.0061(8)$ \\
\hline C3 & $0.0275(11)$ & $0.0150(10)$ & $0.0163(10)$ & $0.0005(8)$ & $0.0064(9)$ & $-0.0045(8)$ \\
\hline C4 & $0.0380(13)$ & $0.0111(10)$ & $0.0193(11)$ & $-0.0015(9)$ & $0.0099(10)$ & $-0.0048(8)$ \\
\hline C5 & $0.0375(13)$ & $0.0163(11)$ & $0.0180(11)$ & $0.0106(9)$ & $0.0055(10)$ & $-0.0055(9)$ \\
\hline C6 & $0.0265(13)$ & $0.0484(16)$ & $0.0306(14)$ & $0.0010(11)$ & $0.0139(11)$ & $-0.0129(12)$ \\
\hline C7 & $0.0456(15)$ & $0.0227(11)$ & $0.0182(11)$ & $-0.0020(10)$ & $0.0131(11)$ & $-0.0009(9)$ \\
\hline C8 & $0.0247(12)$ & $0.0294(13)$ & $0.0269(13)$ & $-0.0002(10)$ & $0.0035(10)$ & $-0.0049(10)$ \\
\hline C9 & $0.0608(18)$ & $0.0202(12)$ & $0.0299(14)$ & $-0.0104(12)$ & $0.0203(13)$ & $-0.0016(10)$ \\
\hline C10 & $0.0539(17)$ & $0.0272(13)$ & $0.0276(14)$ & $0.0213(12)$ & $0.0033(12)$ & $-0.0006(11)$ \\
\hline C11 & $0.0195(10)$ & $0.0177(10)$ & $0.0239(11)$ & $-0.0004(8)$ & $0.0082(9)$ & $0.0015(9)$ \\
\hline C12 & $0.0249(11)$ & $0.0135(10)$ & $0.0177(11)$ & $0.0012(8)$ & $0.0077(9)$ & $0.0036(8)$ \\
\hline C13 & $0.0241(11)$ & $0.0104(9)$ & $0.0174(10)$ & $0.0010(8)$ & $0.0083(9)$ & $0.0049(8)$ \\
\hline C14 & $0.0242(11)$ & $0.0123(9)$ & $0.0177(11)$ & $0.0025(8)$ & $0.0068(9)$ & $0.0049(8)$ \\
\hline C15 & $0.0286(12)$ & $0.0161(10)$ & $0.0178(11)$ & $0.0029(9)$ & $0.0077(9)$ & $0.0020(8)$ \\
\hline C16 & $0.0263(12)$ & $0.0216(11)$ & $0.0184(11)$ & $0.0027(9)$ & $-0.0009(9)$ & $0.0021(9)$ \\
\hline C17 & $0.0195(10)$ & $0.0230(11)$ & $0.0261(12)$ & $0.0051(9)$ & $0.0026(9)$ & $0.0040(9)$ \\
\hline C18 & $0.0220(11)$ & $0.0201(10)$ & $0.0222(11)$ & $0.0049(9)$ & $0.0074(9)$ & $0.0016(9)$ \\
\hline C19 & $0.0231(11)$ & $0.0106(9)$ & $0.0169(10)$ & $0.0015(8)$ & $0.0055(9)$ & $0.0015(8)$ \\
\hline
\end{tabular}




\begin{tabular}{|l|l|l|l|l|l|l|}
\hline N1 & $0.0194(9)$ & $0.0133(8)$ & $0.0176(9)$ & $0.0020(7)$ & $0.0068(7)$ & $0.0023(7)$ \\
\hline Rh1 & $0.01768(10)$ & $0.01182(10)$ & $0.01341(10)$ & $0.00215(6)$ & $0.00479(7)$ & $-0.00007(6)$ \\
\hline
\end{tabular}

Geometric parameters $\left(\AA,{ }^{\circ}\right)$ for (SMK20)

\begin{tabular}{|c|c|c|c|}
\hline $\mathrm{C} 1-\mathrm{C} 5$ & $1.435(4)$ & $\mathrm{C} 9-\mathrm{H} 9 \mathrm{C}$ & 0.9800 \\
\hline $\mathrm{C} 1-\mathrm{C} 2$ & $1.437(3)$ & $\mathrm{C} 10-\mathrm{H} 10 \mathrm{~A}$ & 0.9800 \\
\hline $\mathrm{C} 1-\mathrm{C} 6$ & $1.502(3)$ & $\mathrm{C} 10-\mathrm{H} 10 \mathrm{~B}$ & 0.9800 \\
\hline $\mathrm{C} 1-\mathrm{Rh} 1$ & $2.176(2)$ & $\mathrm{C} 10-\mathrm{H} 10 \mathrm{C}$ & 0.9800 \\
\hline $\mathrm{C} 2-\mathrm{C} 3$ & $1.401(3)$ & $\mathrm{C} 11-\mathrm{N} 1$ & $1.463(3)$ \\
\hline $\mathrm{C} 2-\mathrm{C} 7$ & $1.491(3)$ & C11-H11A & 0.9800 \\
\hline $\mathrm{C} 2-\mathrm{Rh} 1$ & $2.263(2)$ & C11-H11B & 0.9800 \\
\hline $\mathrm{C} 3-\mathrm{C} 4$ & $1.437(3)$ & C11-H11C & 0.9800 \\
\hline $\mathrm{C} 3-\mathrm{C} 8$ & $1.507(3)$ & $\mathrm{C} 12-\mathrm{C} 13$ & $1.462(3)$ \\
\hline $\mathrm{C} 3-\mathrm{Rh} 1$ & $2.287(2)$ & $\mathrm{C} 12-\mathrm{Rh} 1^{\mathrm{i}}$ & $2.177(2)$ \\
\hline $\mathrm{C} 4-\mathrm{C} 5$ & $1.428(4)$ & $\mathrm{C} 12-\mathrm{H} 12 \mathrm{~A}$ & 0.9900 \\
\hline $\mathrm{C} 4-\mathrm{C} 9$ & $1.497(3)$ & C12-H12B & 0.9900 \\
\hline $\mathrm{C} 4-\mathrm{Rh} 1$ & 2.244 (2) & $\mathrm{C} 13-\mathrm{N} 1$ & 1.307 (3) \\
\hline $\mathrm{C} 5-\mathrm{C} 10$ & $1.501(3)$ & $\mathrm{C} 13-\mathrm{C} 14$ & $1.475(3)$ \\
\hline $\mathrm{C} 5-\mathrm{Rh} 1$ & $2.219(2)$ & $\mathrm{C} 14-\mathrm{C} 15$ & $1.398(3)$ \\
\hline C6-H6A & 0.9800 & $\mathrm{C} 14-\mathrm{C} 19$ & $1.411(3)$ \\
\hline C6-H6B & 0.9800 & $\mathrm{C} 15-\mathrm{C} 16$ & $1.388(3)$ \\
\hline $\mathrm{C} 6-\mathrm{H} 6 \mathrm{C}$ & 0.9800 & C15-H15 & 0.9500 \\
\hline C7-H7A & 0.9800 & $\mathrm{C} 16-\mathrm{C} 17$ & $1.386(3)$ \\
\hline C7-H7B & 0.9800 & C16-H16 & 0.9500 \\
\hline $\mathrm{C} 7-\mathrm{H} 7 \mathrm{C}$ & 0.9800 & $\mathrm{C} 17-\mathrm{C} 18$ & $1.387(3)$ \\
\hline $\mathrm{C} 8-\mathrm{H} 8 \mathrm{~A}$ & 0.9800 & C17-H17 & 0.9500 \\
\hline $\mathrm{C} 8-\mathrm{H} 8 \mathrm{~B}$ & 0.9800 & $\mathrm{C} 18-\mathrm{C} 19$ & $1.403(3)$ \\
\hline $\mathrm{C} 8-\mathrm{H} 8 \mathrm{C}$ & 0.9800 & C18-H18 & 0.9500 \\
\hline C9-H9A & 0.9800 & C19-Rh1 & $2.004(2)$ \\
\hline C9-H9B & 0.9800 & $\mathrm{~N} 1-\mathrm{Rh} 1$ & $2.0793(17)$ \\
\hline $\mathrm{C} 5-\mathrm{C} 1-\mathrm{C} 2$ & $107.5(2)$ & $\mathrm{H} 11 \mathrm{~A}-\mathrm{C} 11-\mathrm{H} 11 \mathrm{~B}$ & 109.5 \\
\hline $\mathrm{C} 5-\mathrm{C} 1-\mathrm{C} 6$ & $127.4(2)$ & $\mathrm{N} 1-\mathrm{C} 11-\mathrm{H} 11 \mathrm{C}$ & 109.5 \\
\hline $\mathrm{C} 2-\mathrm{C} 1-\mathrm{C} 6$ & $124.3(2)$ & $\mathrm{H} 11 \mathrm{~A}-\mathrm{C} 11-\mathrm{H} 11 \mathrm{C}$ & 109.5 \\
\hline $\mathrm{C} 5-\mathrm{C} 1-\mathrm{Rh} 1$ & $72.56(12)$ & $\mathrm{H} 11 \mathrm{~B}-\mathrm{C} 11-\mathrm{H} 11 \mathrm{C}$ & 109.5 \\
\hline $\mathrm{C} 2-\mathrm{C} 1-\mathrm{Rh} 1$ & $74.42(12)$ & $\mathrm{C} 13-\mathrm{C} 12-\mathrm{Rh} 1^{\mathrm{i}}$ & $109.81(14)$ \\
\hline $\mathrm{C} 6-\mathrm{C} 1-\mathrm{Rh} 1$ & $126.77(16)$ & $\mathrm{C} 13-\mathrm{C} 12-\mathrm{H} 12 \mathrm{~A}$ & 109.7 \\
\hline
\end{tabular}




\begin{tabular}{|c|c|c|c|}
\hline $\mathrm{C} 3-\mathrm{C} 2-\mathrm{C} 1$ & $108.7(2)$ & $\mathrm{Rh} 11^{\mathrm{i}}-\mathrm{C} 12-\mathrm{H} 12 \mathrm{~A}$ & 109.7 \\
\hline $\mathrm{C} 3-\mathrm{C} 2-\mathrm{C} 7$ & $127.0(2)$ & $\mathrm{C} 13-\mathrm{C} 12-\mathrm{H} 12 \mathrm{~B}$ & 109.7 \\
\hline $\mathrm{C} 1-\mathrm{C} 2-\mathrm{C} 7$ & $124.2(2)$ & $\mathrm{Rh} 11^{\mathrm{i}}-\mathrm{C} 12-\mathrm{H} 12 \mathrm{~B}$ & 109.7 \\
\hline $\mathrm{C} 3-\mathrm{C} 2-\mathrm{Rh} 1$ & $73.02(12)$ & $\mathrm{H} 12 \mathrm{~A}-\mathrm{C} 12-\mathrm{H} 12 \mathrm{~B}$ & 108.2 \\
\hline $\mathrm{C} 1-\mathrm{C} 2-\mathrm{Rh} 1$ & $67.87(12)$ & $\mathrm{N} 1-\mathrm{C} 13-\mathrm{C} 12$ & $126.5(2)$ \\
\hline $\mathrm{C} 7-\mathrm{C} 2-\mathrm{Rh} 1$ & $127.43(15)$ & $\mathrm{N} 1-\mathrm{C} 13-\mathrm{C} 14$ & $112.17(18)$ \\
\hline $\mathrm{C} 2-\mathrm{C} 3-\mathrm{C} 4$ & $108.1(2)$ & $\mathrm{C} 12-\mathrm{C} 13-\mathrm{C} 14$ & $121.02(19)$ \\
\hline $\mathrm{C} 2-\mathrm{C} 3-\mathrm{C} 8$ & $125.7(2)$ & $\mathrm{C} 15-\mathrm{C} 14-\mathrm{C} 19$ & $121.1(2)$ \\
\hline $\mathrm{C} 4-\mathrm{C} 3-\mathrm{C} 8$ & $126.0(2)$ & $\mathrm{C} 15-\mathrm{C} 14-\mathrm{C} 13$ & $124.11(19)$ \\
\hline $\mathrm{C} 2-\mathrm{C} 3-\mathrm{Rh} 1$ & $71.13(12)$ & $\mathrm{C} 19-\mathrm{C} 14-\mathrm{C} 13$ & $114.75(19)$ \\
\hline $\mathrm{C} 4-\mathrm{C} 3-\mathrm{Rh} 1$ & $69.88(12)$ & $\mathrm{C} 16-\mathrm{C} 15-\mathrm{C} 14$ & $120.1(2)$ \\
\hline $\mathrm{C} 8-\mathrm{C} 3-\mathrm{Rh} 1$ & $128.43(15)$ & C16-C15-H15 & 120.0 \\
\hline $\mathrm{C} 5-\mathrm{C} 4-\mathrm{C} 3$ & $108.0(2)$ & $\mathrm{C} 14-\mathrm{C} 15-\mathrm{H} 15$ & 120.0 \\
\hline $\mathrm{C} 5-\mathrm{C} 4-\mathrm{C} 9$ & $126.7(2)$ & $\mathrm{C} 17-\mathrm{C} 16-\mathrm{C} 15$ & $119.5(2)$ \\
\hline $\mathrm{C} 3-\mathrm{C} 4-\mathrm{C} 9$ & $125.0(2)$ & $\mathrm{C} 17-\mathrm{C} 16-\mathrm{H} 16$ & 120.2 \\
\hline $\mathrm{C} 5-\mathrm{C} 4-\mathrm{Rh} 1$ & $70.40(12)$ & $\mathrm{C} 15-\mathrm{C} 16-\mathrm{H} 16$ & 120.2 \\
\hline $\mathrm{C} 3-\mathrm{C} 4-\mathrm{Rh} 1$ & $73.15(12)$ & $\mathrm{C} 16-\mathrm{C} 17-\mathrm{C} 18$ & $120.7(2)$ \\
\hline $\mathrm{C} 9-\mathrm{C} 4-\mathrm{Rh} 1$ & $126.64(16)$ & $\mathrm{C} 16-\mathrm{C} 17-\mathrm{H} 17$ & 119.7 \\
\hline $\mathrm{C} 4-\mathrm{C} 5-\mathrm{C} 1$ & 107.5 & $\mathrm{C} 18-\mathrm{C} 17-\mathrm{H} 17$ & 119.7 \\
\hline $\mathrm{C} 4-\mathrm{C} 5-\mathrm{C} 10$ & $126.5(2)$ & $\mathrm{C} 17-\mathrm{C} 18-\mathrm{C} 19$ & $121.3(2)$ \\
\hline $\mathrm{C} 1-\mathrm{C} 5-\mathrm{C} 10$ & $125.9(2)$ & $\mathrm{C} 17-\mathrm{C} 18-\mathrm{H} 18$ & 119.4 \\
\hline $\mathrm{C} 4-\mathrm{C} 5-\mathrm{Rh} 1$ & $72.29(12)$ & $\mathrm{C} 19-\mathrm{C} 18-\mathrm{H} 18$ & 119.4 \\
\hline $\mathrm{C} 1-\mathrm{C} 5-\mathrm{Rh} 1$ & $69.34(12)$ & $\mathrm{C} 18-\mathrm{C} 19-\mathrm{C} 14$ & $117.3(2)$ \\
\hline $\mathrm{C} 10-\mathrm{C} 5-\mathrm{Rh} 1$ & $126.56(16)$ & $\mathrm{C} 18-\mathrm{C} 19-\mathrm{Rh} 1$ & $127.37(16)$ \\
\hline $\mathrm{C} 1-\mathrm{C} 6-\mathrm{H} 6 \mathrm{~A}$ & 109.5 & $\mathrm{C} 14-\mathrm{C} 19-\mathrm{Rh} 1$ & $115.29(16)$ \\
\hline $\mathrm{C} 1-\mathrm{C} 6-\mathrm{H} 6 \mathrm{~B}$ & 109.5 & $\mathrm{C} 13-\mathrm{N} 1-\mathrm{C} 11$ & $121.23(18)$ \\
\hline $\mathrm{H} 6 \mathrm{~A}-\mathrm{C} 6-\mathrm{H} 6 \mathrm{~B}$ & 109.5 & $\mathrm{C} 13-\mathrm{N} 1-\mathrm{Rh} 1$ & $117.53(14)$ \\
\hline $\mathrm{C} 1-\mathrm{C} 6-\mathrm{H} 6 \mathrm{C}$ & 109.5 & $\mathrm{C} 11-\mathrm{N} 1-\mathrm{Rh} 1$ & $120.97(14)$ \\
\hline $\mathrm{H} 6 \mathrm{~A}-\mathrm{C} 6-\mathrm{H} 6 \mathrm{C}$ & 109.5 & $\mathrm{C} 19-\mathrm{Rh} 1-\mathrm{N} 1$ & $77.95(8)$ \\
\hline $\mathrm{H} 6 \mathrm{~B}-\mathrm{C} 6-\mathrm{H} 6 \mathrm{C}$ & 109.5 & $\mathrm{C} 19-\mathrm{Rh} 1-\mathrm{C} 1$ & $107.72(9)$ \\
\hline $\mathrm{C} 2-\mathrm{C} 7-\mathrm{H} 7 \mathrm{~A}$ & 109.5 & $\mathrm{~N} 1-\mathrm{Rh} 1-\mathrm{C} 1$ & $166.14(8)$ \\
\hline $\mathrm{C} 2-\mathrm{C} 7-\mathrm{H} 7 \mathrm{~B}$ & 109.5 & $\mathrm{C} 19-\mathrm{Rh} 1-\mathrm{C} 12^{\mathrm{i}}$ & $85.06(8)$ \\
\hline $\mathrm{H} 7 \mathrm{~A}-\mathrm{C} 7-\mathrm{H} 7 \mathrm{~B}$ & 109.5 & $\mathrm{~N} 1-\mathrm{Rh} 1-\mathrm{C} 12^{\mathrm{i}}$ & $91.49(7)$ \\
\hline $\mathrm{C} 2-\mathrm{C} 7-\mathrm{H} 7 \mathrm{C}$ & 109.5 & $\mathrm{C} 1-\mathrm{Rh} 1-\mathrm{C} 12^{\mathrm{i}}$ & $101.49(9)$ \\
\hline $\mathrm{H} 7 \mathrm{~A}-\mathrm{C} 7-\mathrm{H} 7 \mathrm{C}$ & 109.5 & $\mathrm{C} 19-\mathrm{Rh} 1-\mathrm{C} 5$ & 99.07 (8) \\
\hline $\mathrm{H} 7 \mathrm{~B}-\mathrm{C} 7-\mathrm{H} 7 \mathrm{C}$ & 109.5 & $\mathrm{~N} 1-\mathrm{Rh} 1-\mathrm{C} 5$ & $129.53(8)$ \\
\hline $\mathrm{C} 3-\mathrm{C} 8-\mathrm{H} 8 \mathrm{~A}$ & 109.5 & $\mathrm{C} 1-\mathrm{Rh} 1-\mathrm{C} 5$ & $38.10(9)$ \\
\hline
\end{tabular}




\begin{tabular}{|c|c|c|c|}
\hline $\mathrm{C} 3-\mathrm{C} 8-\mathrm{H} 8 \mathrm{~B}$ & 109.5 & $\mathrm{C} 12^{\mathrm{i}}-\mathrm{Rh} 1-\mathrm{C} 5$ & $138.86(9)$ \\
\hline $\mathrm{H} 8 \mathrm{~A}-\mathrm{C} 8-\mathrm{H} 8 \mathrm{~B}$ & 109.5 & $\mathrm{C} 19-\mathrm{Rh} 1-\mathrm{C} 4$ & $123.54(8)$ \\
\hline $\mathrm{C} 3-\mathrm{C} 8-\mathrm{H} 8 \mathrm{C}$ & 109.5 & $\mathrm{~N} 1-\mathrm{Rh} 1-\mathrm{C} 4$ & $103.27(8)$ \\
\hline $\mathrm{H} 8 \mathrm{~A}-\mathrm{C} 8-\mathrm{H} 8 \mathrm{C}$ & 109.5 & $\mathrm{C} 1-\mathrm{Rh} 1-\mathrm{C} 4$ & $62.96(9)$ \\
\hline $\mathrm{H} 8 \mathrm{~B}-\mathrm{C} 8-\mathrm{H} 8 \mathrm{C}$ & 109.5 & $\mathrm{C} 12^{\mathrm{i}}-\mathrm{Rh} 1-\mathrm{C} 4$ & $149.73(8)$ \\
\hline $\mathrm{C} 4-\mathrm{C} 9-\mathrm{H} 9 \mathrm{~A}$ & 109.5 & C5-Rh1-C4 & $37.31(9)$ \\
\hline $\mathrm{C} 4-\mathrm{C} 9-\mathrm{H} 9 \mathrm{~B}$ & 109.5 & $\mathrm{C} 19-\mathrm{Rh} 1-\mathrm{C} 2$ & $143.08(9)$ \\
\hline $\mathrm{H} 9 \mathrm{~A}-\mathrm{C} 9-\mathrm{H} 9 \mathrm{~B}$ & 109.5 & $\mathrm{~N} 1-\mathrm{Rh} 1-\mathrm{C} 2$ & $138.86(8)$ \\
\hline $\mathrm{C} 4-\mathrm{C} 9-\mathrm{H} 9 \mathrm{C}$ & 109.5 & $\mathrm{C} 1-\mathrm{Rh} 1-\mathrm{C} 2$ & $37.71(8)$ \\
\hline $\mathrm{H} 9 \mathrm{~A}-\mathrm{C} 9-\mathrm{H} 9 \mathrm{C}$ & 109.5 & $\mathrm{C} 12^{\mathrm{i}}-\mathrm{Rh} 1-\mathrm{C} 2$ & $90.20(8)$ \\
\hline $\mathrm{H} 9 \mathrm{~B}-\mathrm{C} 9-\mathrm{H} 9 \mathrm{C}$ & 109.5 & $\mathrm{C} 5-\mathrm{Rh} 1-\mathrm{C} 2$ & $62.21(8)$ \\
\hline $\mathrm{C} 5-\mathrm{C} 10-\mathrm{H} 10 \mathrm{~A}$ & 109.5 & $\mathrm{C} 4-\mathrm{Rh} 1-\mathrm{C} 2$ & $61.32(8)$ \\
\hline $\mathrm{C} 5-\mathrm{C} 10-\mathrm{H} 10 \mathrm{~B}$ & 109.5 & $\mathrm{C} 19-\mathrm{Rh} 1-\mathrm{C} 3$ & $159.86(8)$ \\
\hline $\mathrm{H} 10 \mathrm{~A}-\mathrm{C} 10-\mathrm{H} 10 \mathrm{~B}$ & 109.5 & $\mathrm{~N} 1-\mathrm{Rh} 1-\mathrm{C} 3$ & $108.30(7)$ \\
\hline $\mathrm{C} 5-\mathrm{C} 10-\mathrm{H} 10 \mathrm{C}$ & 109.5 & $\mathrm{C} 1-\mathrm{Rh} 1-\mathrm{C} 3$ & $62.14(8)$ \\
\hline $\mathrm{H} 10 \mathrm{~A}-\mathrm{C} 10-\mathrm{H} 10 \mathrm{C}$ & 109.5 & $\mathrm{C} 12^{\mathrm{i}}-\mathrm{Rh} 1-\mathrm{C} 3$ & $113.34(8)$ \\
\hline $\mathrm{H} 10 \mathrm{~B}-\mathrm{C} 10-\mathrm{H} 10 \mathrm{C}$ & 109.5 & $\mathrm{C} 5-\mathrm{Rh} 1-\mathrm{C} 3$ & $61.90(8)$ \\
\hline $\mathrm{N} 1-\mathrm{C} 11-\mathrm{H} 11 \mathrm{~A}$ & 109.5 & $\mathrm{C} 4-\mathrm{Rh} 1-\mathrm{C} 3$ & $36.97(8)$ \\
\hline $\mathrm{N} 1-\mathrm{C} 11-\mathrm{H} 11 \mathrm{~B}$ & 109.5 & $\mathrm{C} 2-\mathrm{Rh} 1-\mathrm{C} 3$ & $35.85(8)$ \\
\hline $\mathrm{C} 5-\mathrm{C} 1-\mathrm{C} 2-\mathrm{C} 3$ & $-3.6(2)$ & $\mathrm{C} 9-\mathrm{C} 4-\mathrm{C} 5-\mathrm{Rh} 1$ & $121.7(2)$ \\
\hline $\mathrm{C} 6-\mathrm{C} 1-\mathrm{C} 2-\mathrm{C} 3$ & $-173.9(2)$ & $\mathrm{C} 2-\mathrm{C} 1-\mathrm{C} 5-\mathrm{C} 4$ & $4.2(2)$ \\
\hline $\mathrm{Rh} 1-\mathrm{C} 1-\mathrm{C} 2-\mathrm{C} 3$ & $61.95(15)$ & $\mathrm{C} 6-\mathrm{C} 1-\mathrm{C} 5-\mathrm{C} 4$ & $174.0(2)$ \\
\hline $\mathrm{C} 5-\mathrm{C} 1-\mathrm{C} 2-\mathrm{C} 7$ & $173.32(19)$ & $\mathrm{Rh} 1-\mathrm{C} 1-\mathrm{C} 5-\mathrm{C} 4$ & $-62.63(14)$ \\
\hline $\mathrm{C} 6-\mathrm{C} 1-\mathrm{C} 2-\mathrm{C} 7$ & $3.1(3)$ & $\mathrm{C} 2-\mathrm{C} 1-\mathrm{C} 5-\mathrm{C} 10$ & $-172.3(2)$ \\
\hline $\mathrm{Rh} 1-\mathrm{C} 1-\mathrm{C} 2-\mathrm{C} 7$ & $-121.1(2)$ & $\mathrm{C} 6-\mathrm{C} 1-\mathrm{C} 5-\mathrm{C} 10$ & $-2.5(4)$ \\
\hline $\mathrm{C} 5-\mathrm{C} 1-\mathrm{C} 2-\mathrm{Rh} 1$ & $-65.55(14)$ & $\mathrm{Rh} 1-\mathrm{C} 1-\mathrm{C} 5-\mathrm{C} 10$ & $120.9(2)$ \\
\hline $\mathrm{C} 6-\mathrm{C} 1-\mathrm{C} 2-\mathrm{Rh} 1$ & $124.2(2)$ & $\mathrm{C} 2-\mathrm{C} 1-\mathrm{C} 5-\mathrm{Rh} 1$ & $66.80(14)$ \\
\hline $\mathrm{C} 1-\mathrm{C} 2-\mathrm{C} 3-\mathrm{C} 4$ & $1.6(2)$ & $\mathrm{C} 6-\mathrm{C} 1-\mathrm{C} 5-\mathrm{Rh} 1$ & $-123.3(2)$ \\
\hline $\mathrm{C} 7-\mathrm{C} 2-\mathrm{C} 3-\mathrm{C} 4$ & $-175.2(2)$ & $\mathrm{Rh} 1{ }^{\mathrm{i}}-\mathrm{C} 12-\mathrm{C} 13-\mathrm{N} 1$ & $96.1(2)$ \\
\hline $\mathrm{Rh} 1-\mathrm{C} 2-\mathrm{C} 3-\mathrm{C} 4$ & $60.35(14)$ & $\begin{array}{l}\mathrm{Rh} 11^{\mathrm{i}}-\mathrm{C} 12-\mathrm{C} 13- \\
\mathrm{C} 14\end{array}$ & $-76.7(2)$ \\
\hline $\mathrm{C} 1-\mathrm{C} 2-\mathrm{C} 3-\mathrm{C} 8$ & $177.0(2)$ & $\mathrm{N} 1-\mathrm{C} 13-\mathrm{C} 14-\mathrm{C} 15$ & $168.51(19)$ \\
\hline $\mathrm{C} 7-\mathrm{C} 2-\mathrm{C} 3-\mathrm{C} 8$ & $0.2(3)$ & $\begin{array}{l}\text { C12-C13-C14- } \\
\text { C15 }\end{array}$ & $-17.7(3)$ \\
\hline $\mathrm{Rh} 1-\mathrm{C} 2-\mathrm{C} 3-\mathrm{C} 8$ & $-124.3(2)$ & $\mathrm{N} 1-\mathrm{C} 13-\mathrm{C} 14-\mathrm{C} 19$ & $-10.1(3)$ \\
\hline $\mathrm{C} 1-\mathrm{C} 2-\mathrm{C} 3-\mathrm{Rh} 1$ & $-58.74(14)$ & $\begin{array}{l}\mathrm{C} 12-\mathrm{C} 13-\mathrm{C} 14- \\
\mathrm{C} 19\end{array}$ & $163.68(19)$ \\
\hline
\end{tabular}




\begin{tabular}{|c|c|c|c|}
\hline $\mathrm{C} 7-\mathrm{C} 2-\mathrm{C} 3-\mathrm{Rh} 1$ & $124.4(2)$ & $\begin{array}{l}\mathrm{C} 19-\mathrm{C} 14-\mathrm{C} 15- \\
\mathrm{C} 16\end{array}$ & $-0.4(3)$ \\
\hline $\mathrm{C} 2-\mathrm{C} 3-\mathrm{C} 4-\mathrm{C} 5$ & $1.0(2)$ & $\begin{array}{l}\mathrm{C} 13-\mathrm{C} 14-\mathrm{C} 15- \\
\mathrm{C} 16\end{array}$ & $-179.0(2)$ \\
\hline $\mathrm{C} 8-\mathrm{C} 3-\mathrm{C} 4-\mathrm{C} 5$ & $-174.3(2)$ & $\begin{array}{l}\mathrm{C} 14-\mathrm{C} 15-\mathrm{C} 16- \\
\mathrm{C} 17\end{array}$ & $-0.6(3)$ \\
\hline $\mathrm{Rh} 1-\mathrm{C} 3-\mathrm{C} 4-\mathrm{C} 5$ & $62.15(14)$ & $\begin{array}{l}\mathrm{C} 15-\mathrm{C} 16-\mathrm{C} 17- \\
\mathrm{C} 18\end{array}$ & $0.4(3)$ \\
\hline $\mathrm{C} 2-\mathrm{C} 3-\mathrm{C} 4-\mathrm{C} 9$ & $175.5(2)$ & $\begin{array}{l}\mathrm{C} 16-\mathrm{C} 17-\mathrm{C} 18- \\
\mathrm{C} 19\end{array}$ & $0.8(4)$ \\
\hline $\mathrm{C} 8-\mathrm{C} 3-\mathrm{C} 4-\mathrm{C} 9$ & $0.2(3)$ & $\begin{array}{l}\mathrm{C} 17-\mathrm{C} 18-\mathrm{C} 19- \\
\mathrm{C} 14\end{array}$ & $-1.7(3)$ \\
\hline $\mathrm{Rh} 1-\mathrm{C} 3-\mathrm{C} 4-\mathrm{C} 9$ & $-123.3(2)$ & $\begin{array}{l}\text { C17-C18-C19- } \\
\text { Rh1 }\end{array}$ & $179.91(17)$ \\
\hline $\mathrm{C} 2-\mathrm{C} 3-\mathrm{C} 4-\mathrm{Rh} 1$ & $-61.14(15)$ & $\begin{array}{l}\mathrm{C} 15-\mathrm{C} 14-\mathrm{C} 19- \\
\mathrm{C} 18\end{array}$ & $1.6(3)$ \\
\hline $\mathrm{C} 8-\mathrm{C} 3-\mathrm{C} 4-\mathrm{Rh} 1$ & $123.5(2)$ & $\begin{array}{l}\mathrm{C} 13-\mathrm{C} 14-\mathrm{C} 19- \\
\mathrm{C} 18\end{array}$ & $-179.78(18)$ \\
\hline $\mathrm{C} 3-\mathrm{C} 4-\mathrm{C} 5-\mathrm{C} 1$ & $-3.2(2)$ & $\begin{array}{l}\text { C15-C14-C19- } \\
\text { Rh1 }\end{array}$ & $-179.89(16)$ \\
\hline $\mathrm{C} 9-\mathrm{C} 4-\mathrm{C} 5-\mathrm{C} 1$ & $-177.6(2)$ & $\begin{array}{l}\text { C13-C14-C19- } \\
\text { Rh1 }\end{array}$ & $-1.2(2)$ \\
\hline $\mathrm{Rh} 1-\mathrm{C} 4-\mathrm{C} 5-\mathrm{C} 1$ & $60.72(14)$ & $\mathrm{C} 12-\mathrm{C} 13-\mathrm{N} 1-\mathrm{C} 11$ & $17.5(3)$ \\
\hline $\mathrm{C} 3-\mathrm{C} 4-\mathrm{C} 5-\mathrm{C} 10$ & $173.3(2)$ & $\mathrm{C} 14-\mathrm{C} 13-\mathrm{N} 1-\mathrm{C} 11$ & $-169.14(18)$ \\
\hline $\mathrm{C} 9-\mathrm{C} 4-\mathrm{C} 5-\mathrm{C} 10$ & $-1.1(4)$ & $\mathrm{C} 12-\mathrm{C} 13-\mathrm{N} 1-\mathrm{Rh} 1$ & $-156.58(16)$ \\
\hline $\mathrm{Rh} 1-\mathrm{C} 4-\mathrm{C} 5-\mathrm{C} 10$ & $-122.8(2)$ & $\mathrm{C} 14-\mathrm{C} 13-\mathrm{N} 1-\mathrm{Rh} 1$ & $16.8(2)$ \\
\hline $\mathrm{C} 3-\mathrm{C} 4-\mathrm{C} 5-\mathrm{Rh} 1$ & $-63.93(14)$ & & \\
\hline
\end{tabular}

Symmetry code: (i) $-x+1,-y,-z+1$.

Document origin: publCIF [Westrip, S. P. (2010). J. Apply. Cryst., 43, 920-925].

Compound $\left[\left(\eta^{5}-\mathrm{C}_{5} \mathrm{Me}_{5}\right) \mathrm{Rh}\left({ }^{\mathrm{Me}} \mathrm{PhI}\right)(\mathrm{MeCN})\right]\left[\mathrm{BArF}_{24}\right]$ : 


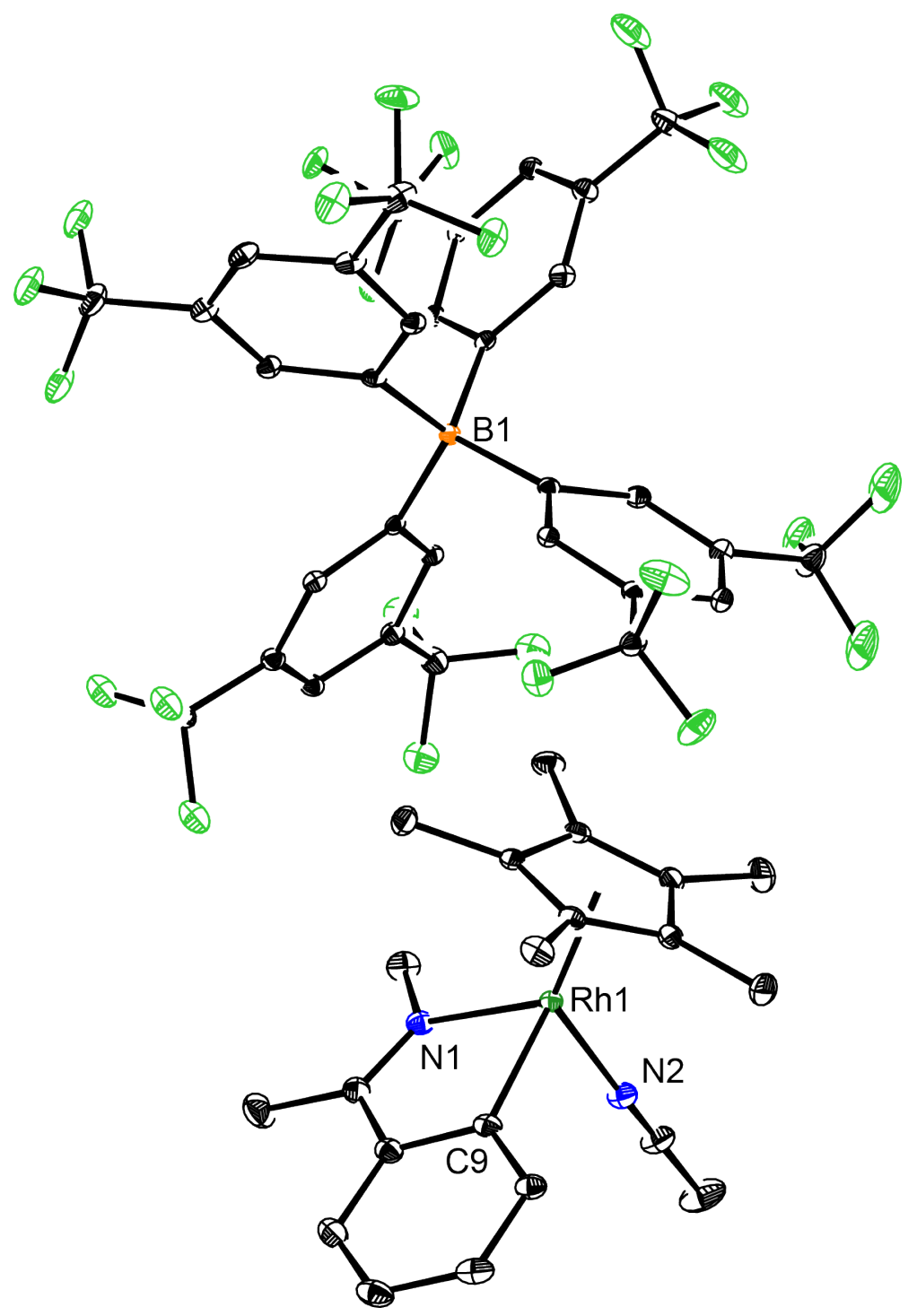

Table S5

Experimental details

\begin{tabular}{|c|c|}
\hline \multicolumn{2}{|l|}{ Crystal data } \\
\hline Chemical formula & $\mathrm{C}_{53} \mathrm{H}_{40} \mathrm{BF}_{24} \mathrm{~N}_{2} \mathrm{Rh}$ \\
\hline$M_{\mathrm{r}}$ & 1274.59 \\
\hline Crystal system, space group & Triclinic, $P^{-}{ }_{1}$ \\
\hline Temperature (K) & 100 \\
\hline$a, b, c(\AA)$ & $12.8596(2), 12.8714(2), 16.7648(2)$ \\
\hline$\alpha, \beta, \gamma\left(^{\circ}\right)$ & $92.496(1), 96.418(1), 105.15$ \\
\hline$V\left(\AA^{3}\right)$ & $2654.01(7)$ \\
\hline
\end{tabular}




\begin{tabular}{|l|l|}
\hline$Z$ & 2 \\
\hline Radiation type & Cu $K \alpha$ \\
\hline$\mu\left(\mathrm{mm}^{-1}\right)$ & 3.73 \\
\hline Crystal size $(\mathrm{mm})$ & $0.24 \times 0.17 \times 0.12$ \\
\hline \multicolumn{2}{|l|}{} \\
\hline Data collection & Bruker $A P E X$-II CCD \\
\hline Diffractometer & $\begin{array}{l}\text { Multi-scan } \\
\text { Absorption correction }\end{array}$ \\
\hline$T_{\min ,}, T_{\max }$ & $0.639,0.753$ \\
\hline $\begin{array}{l}\text { No. of measured, independent and } \\
\text { observed }[I>2 \sigma(I)] \text { reflections }\end{array}$ & $45300,9193,9181$ \\
\hline$R_{\text {int }}$ & \\
\hline$(\text { sin } \theta / \lambda)_{\max }\left(\AA^{-1}\right)$ & 0.018 \\
\hline & 0.595 \\
\hline Refinement & \multicolumn{2}{|l}{} \\
\hline$R\left[F^{2}>2 \sigma\left(F^{2}\right)\right], w R\left(F^{2}\right), S$ & $0.047,0.116,1.10$ \\
\hline No. of reflections & 9193 \\
\hline No. of parameters & 744 \\
\hline H-atom treatment & H-atom parameters constrained \\
\hline$\left.\Delta\rangle_{\max }, \Delta\right\rangle_{\min }\left(\mathrm{e} \AA^{-3}\right)$ & $1.55,-1.27$ \\
\hline &
\end{tabular}

Computer programs: Bruker APEX2, Bruker SAINT, SHELXT 2014/4 (Sheldrick, 2014), SHELXL2018/3 (Sheldrick, 2018).

Document origin: publCIF [Westrip, S. P. (2010). J. Apply. Cryst., 43, 920-925].

\section{full crystallographic data}

\section{Computing details}

Data collection: Bruker APEX2; cell refinement: Bruker SAINT; data reduction: Bruker SAINT; program(s) used to solve structure: SHELXT 2014/4 (Sheldrick, 2014); program(s) used to refine structure: SHELXL2018/3 (Sheldrick, 2018).

\section{(SMK21)}

\section{Crystal data}

\begin{tabular}{|l|l|}
\hline $\mathrm{C}_{53} \mathrm{H}_{40} \mathrm{BF}_{24} \mathrm{~N}_{2} \mathrm{Rh}$ & $Z=2$ \\
\hline
\end{tabular}




\begin{tabular}{|l|l|}
\hline$M_{r}=1274.59$ & $F(000)=1276$ \\
\hline Triclinic, $P^{-} 1$ & $D_{\mathrm{x}}=1.595 \mathrm{Mg} \mathrm{m}^{-3}$ \\
\hline$a=12.8596(2) \AA$ & Cu Ka radiation, $\lambda=1.54178 \AA$ \\
\hline$b=12.8714(2) \AA$ & Cell parameters from 9735 reflections \\
\hline$c=16.7648(2) \AA$ & $\theta=2.7-66.7^{\circ}$ \\
\hline$\alpha=92.496(1)^{\circ}$ & $\mu=3.73 \mathrm{~mm}^{-1}$ \\
\hline$\beta=96.418(1)^{\circ}$ & $T=100 \mathrm{~K}$ \\
\hline$\gamma=105.15^{\circ}$ & Block, yellow \\
\hline$V=2654.01(7) \AA^{3}$ & $0.24 \times 0.17 \times 0.12 \mathrm{~mm}$ \\
\hline
\end{tabular}

\section{Data collection}

\begin{tabular}{|l|l|}
\hline $\begin{array}{l}\text { Bruker APEX-II CCD } \\
\text { diffractometer }\end{array}$ & 9181 reflections with $I>2 \sigma(I)$ \\
\hline Radiation source: I $\mu$ S microfocus source & $R_{\text {int }}=0.018$ \\
\hline$\phi$ and $\omega$ scans & $\theta_{\max }=66.7^{\circ}, \theta_{\min }=2.7^{\circ}$ \\
\hline $\begin{array}{l}\text { Absorption correction: multi-scan } \\
\text { TWINABS BRUKER AXS }\end{array}$ & $h=-15 \rightarrow 15$ \\
\hline$T_{\min }=0.639, T_{\max }=0.753$ & $k=-15 \rightarrow 15$ \\
\hline 45300 measured reflections & $l=-19 \rightarrow 19$ \\
\hline 9193 independent reflections & \\
\hline
\end{tabular}

\section{Refinement}

\begin{tabular}{|l|l|}
\hline Refinement on $F^{2}$ & 0 restraints \\
\hline Least-squares matrix: full & $\begin{array}{l}\text { Hydrogen site location: inferred from } \\
\text { neighbouring sites }\end{array}$ \\
\hline$R\left[F^{2}>2 \sigma\left(F^{2}\right)\right]=0.047$ & H-atom parameters constrained \\
\hline$w R\left(F^{2}\right)=0.116$ & $\begin{array}{l}w=1 /\left[\sigma^{2}\left(F_{\mathrm{o}}{ }^{2}\right)+(0.0507 P)^{2}+8.9261 P\right] \\
\text { where } P=\left(F_{\mathrm{o}}{ }^{2}+2 F_{\mathrm{c}}{ }^{2}\right) / 3\end{array}$ \\
\hline$S=1.10$ & $(\Delta / \sigma)_{\max }=0.001$ \\
\hline 9193 reflections & $\Delta\rangle_{\max }=1.55 \mathrm{e} \AA^{-3}$ \\
\hline 744 parameters & $\Delta\rangle_{\min }=-1.27 \mathrm{e} \AA^{-3}$ \\
\hline
\end{tabular}

\section{Special details}

Experimental. Collected, solved and refined by Hongyu Zhong

Geometry. All esds (except the esd in the dihedral angle between two 1.s. planes) are estimated using the full covariance matrix. The cell esds are taken into account individually in the estimation of esds in distances, angles and torsion angles; correlations between esds in cell parameters are only used when they are defined by crystal symmetry. An approximate (isotropic) 
treatment of cell esds is used for estimating esds involving 1.s. planes.

Fractional atomic coordinates and isotropic or equivalent isotropic displacement parameters $\left(\AA^{2}\right)$ for (SMK21)

\begin{tabular}{|c|c|c|c|c|c|}
\hline & $x$ & $y$ & $z$ & $U_{\text {iso }} * / U_{\text {eq }}$ & Occ. $(<1)$ \\
\hline $\mathrm{C} 1$ & $1.1016(3)$ & $0.5532(3)$ & $0.7108(2)$ & $0.0274(8)$ & \\
\hline H1A & 1.037969 & 0.569400 & 0.681708 & $0.041 *$ & \\
\hline H1B & 1.162878 & 0.572764 & 0.679417 & $0.041 *$ & \\
\hline $\mathrm{H} 1 \mathrm{C}$ & 1.084920 & 0.475847 & 0.718727 & $0.041 *$ & \\
\hline $\mathrm{C} 2$ & $1.2791(3)$ & $0.5312(4)$ & $0.8236(3)$ & $0.0361(10)$ & \\
\hline $\mathrm{H} 2 \mathrm{~A}$ & 1.336254 & 0.539071 & 0.869054 & $0.054 *$ & \\
\hline $\mathrm{H} 2 \mathrm{~B}$ & 1.232695 & 0.456910 & 0.817710 & $0.054^{*}$ & \\
\hline $\mathrm{H} 2 \mathrm{C}$ & 1.312158 & 0.547803 & 0.774199 & $0.054 *$ & \\
\hline $\mathrm{C} 3$ & $1.2118(3)$ & $0.6075(3)$ & $0.8388(2)$ & $0.0221(7)$ & \\
\hline $\mathrm{C} 4$ & $1.2372(3)$ & $0.6831(3)$ & 0.9109 (2) & $0.0206(7)$ & \\
\hline $\mathrm{C} 5$ & $1.3249(3)$ & $0.6927(3)$ & $0.9706(2)$ & $0.0271(8)$ & \\
\hline H5 & 1.369213 & 0.644309 & 0.968534 & $0.032 *$ & \\
\hline C6 & $1.3469(3)$ & $0.7727(3)$ & $1.0326(2)$ & $0.0293(8)$ & \\
\hline H6 & 1.406042 & 0.778844 & 1.073550 & $0.035^{*}$ & \\
\hline $\mathrm{C} 7$ & $1.2828(3)$ & $0.8441(3)$ & $1.0351(2)$ & $0.0266(8)$ & \\
\hline $\mathrm{H} 7$ & 1.298999 & 0.899781 & 1.077259 & $0.032 *$ & \\
\hline $\mathrm{C} 8$ & $1.1948(3)$ & $0.8345(3)$ & $0.9759(2)$ & $0.0217(7)$ & \\
\hline H8 & 1.151465 & 0.883880 & 0.978134 & $0.026^{*}$ & \\
\hline $\mathrm{C} 9$ & $1.1699(3)$ & $0.7536(3)$ & $0.9140(2)$ & $0.0181(7)$ & \\
\hline $\mathrm{C} 10$ & 0.8704 (3) & $0.6362(3)$ & $0.7744(2)$ & $0.0185(7)$ & \\
\hline C11 & 0.8815 (3) & $0.7475(3)$ & $0.7793(2)$ & $0.0201(7)$ & \\
\hline $\mathrm{C} 12$ & $0.9176(3)$ & $0.7910(3)$ & $0.8621(2)$ & $0.0190(7)$ & \\
\hline $\mathrm{C} 13$ & $0.9237(3)$ & $0.7025(3)$ & $0.9091(2)$ & $0.0181(7)$ & \\
\hline $\mathrm{C} 14$ & $0.9020(3)$ & $0.6080(3)$ & $0.8551(2)$ & $0.0171(7)$ & \\
\hline $\mathrm{C} 15$ & $0.8291(3)$ & $0.5578(3)$ & $0.7020(2)$ & $0.0267(8)$ & \\
\hline $\mathrm{H} 15 \mathrm{~A}$ & 0.830552 & 0.485213 & 0.716703 & $0.040^{*}$ & \\
\hline H15B & 0.754445 & 0.557846 & 0.682448 & $0.040^{*}$ & \\
\hline H15C & 0.875400 & 0.578842 & 0.659627 & $0.040^{*}$ & \\
\hline $\mathrm{C} 16$ & $0.8589(3)$ & $0.8136(3)$ & $0.7113(2)$ & $0.0321(9)$ & \\
\hline H16A & 0.873389 & 0.889257 & 0.731565 & $0.048^{*}$ & \\
\hline H16B & 0.905847 & 0.808084 & 0.670013 & $0.048^{*}$ & \\
\hline $\mathrm{H} 16 \mathrm{C}$ & 0.782625 & 0.786709 & 0.687838 & $0.048 *$ & \\
\hline
\end{tabular}




\begin{tabular}{|c|c|c|c|c|}
\hline $\mathrm{C} 17$ & $0.9267(3)$ & $0.9044(3)$ & $0.8924(2)$ & $0.0284(8)$ \\
\hline H17A & 0.919410 & 0.947936 & 0.846603 & $0.043^{*}$ \\
\hline H17B & 0.869046 & 0.904870 & 0.925858 & $0.043 *$ \\
\hline $\mathrm{H} 17 \mathrm{C}$ & 0.997665 & 0.934701 & 0.924468 & $0.043 *$ \\
\hline $\mathrm{C} 18$ & $0.9448(3)$ & $0.7055(3)$ & $0.9990(2)$ & $0.0245(8)$ \\
\hline H18A & 0.944611 & 0.633156 & 1.015131 & $0.037^{*}$ \\
\hline H18B & 1.015593 & 0.755894 & 1.018006 & $0.037^{*}$ \\
\hline $\mathrm{H} 18 \mathrm{C}$ & 0.887818 & 0.729310 & 1.022761 & $0.037 *$ \\
\hline C19 & $0.8983(3)$ & $0.4961(3)$ & $0.8787(2)$ & $0.0257(8)$ \\
\hline H19A & 0.881887 & 0.446277 & 0.830324 & $0.039 *$ \\
\hline H19B & 0.968818 & 0.495672 & 0.907490 & $0.039 *$ \\
\hline H19C & 0.841724 & 0.473381 & 0.913818 & $0.039^{*}$ \\
\hline $\mathrm{C} 20$ & $0.4275(3)$ & $0.2298(2)$ & $0.61744(19)$ & $0.0132(6)$ \\
\hline $\mathrm{C} 21$ & $0.4314(3)$ & $0.1544(2)$ & $0.55630(19)$ & $0.0143(6)$ \\
\hline $\mathrm{H} 21$ & 0.496100 & 0.132254 & 0.554503 & $0.017 *$ \\
\hline $\mathrm{C} 22$ & $0.3427(3)$ & $0.1105(2)$ & 0.49767 (19) & $0.0149(6)$ \\
\hline $\mathrm{C} 23$ & $0.2473(3)$ & $0.1404(3)$ & $0.4976(2)$ & $0.0170(7)$ \\
\hline $\mathrm{H} 23$ & 0.186996 & 0.109902 & 0.457771 & $0.020^{*}$ \\
\hline $\mathrm{C} 24$ & $0.2418(3)$ & $0.2166(3)$ & $0.5575(2)$ & $0.0192(7)$ \\
\hline $\mathrm{C} 25$ & 0.3301 & $0.2598(3)$ & $0.6160(2)$ & $0.0170(7)$ \\
\hline $\mathrm{H} 25$ & 0.324271 & 0.311402 & 0.656267 & $0.020^{*}$ \\
\hline $\mathrm{C} 26$ & $0.3502(3)$ & $0.0257(3)$ & $0.4363(2)$ & $0.0195(7)$ \\
\hline $\mathrm{C} 27$ & $0.1397(3)$ & $0.2512(3)$ & $0.5567(2)$ & $0.0281(8)$ \\
\hline $\mathrm{C} 28$ & $0.6454(3)$ & $0.2708(2)$ & $0.67084(19)$ & $0.0125(6)$ \\
\hline $\mathrm{C} 29$ & $0.7244(3)$ & $0.2444(2)$ & $0.7251(2)$ & $0.0148(6)$ \\
\hline $\mathrm{H} 29$ & 0.706688 & 0.223045 & 0.776496 & $0.018^{*}$ \\
\hline $\mathrm{C} 30$ & $0.8278(3)$ & $0.2486(3)$ & $0.7056(2)$ & $0.0172(7)$ \\
\hline $\mathrm{C} 31$ & $0.8584(3)$ & $0.2835(3)$ & $0.6326(2)$ & $0.0175(7)$ \\
\hline $\mathrm{H} 31$ & 0.928973 & 0.286205 & 0.619386 & $0.021 *$ \\
\hline $\mathrm{C} 32$ & $0.7834(3)$ & $0.3145(3)$ & $0.5791(2)$ & $0.0172(7)$ \\
\hline $\mathrm{C} 33$ & $0.6788(3)$ & $0.3067(2)$ & $0.59781(19)$ & $0.0145(6)$ \\
\hline H33 & 0.628352 & 0.326545 & 0.559648 & $0.017^{*}$ \\
\hline $\mathrm{C} 34$ & $0.9083(3)$ & $0.2132(3)$ & $0.7622(2)$ & $0.0215(7)$ \\
\hline $\mathrm{C} 35$ & $0.8148(3)$ & $0.3532(3)$ & $0.5002(2)$ & $0.0236(7)$ \\
\hline $\mathrm{C} 36$ & $0.4826(2)$ & $0.1857(3)$ & $0.76082(19)$ & $0.0136(6)$ \\
\hline $\mathrm{C} 37$ & $0.4036(3)$ & $0.2003(3)$ & $0.80829(19)$ & $0.0150(6)$ \\
\hline H37 & 0.381167 & 0.264952 & 0.805871 & $0.018^{*}$ \\
\hline
\end{tabular}




\begin{tabular}{|c|c|c|c|c|c|}
\hline $\mathrm{C} 38$ & $0.3570(3)$ & $0.1233(3)$ & $0.8587(2)$ & $0.0185(7)$ & \\
\hline C39 & $0.3882(3)$ & $0.0282(3)$ & $0.8653(2)$ & $0.0207(7)$ & \\
\hline H39 & 0.357656 & -0.023589 & 0.900777 & $0.025^{*}$ & \\
\hline $\mathrm{C} 40$ & $0.4656(3)$ & $0.0115(3)$ & $0.8184(2)$ & $0.0202(7)$ & \\
\hline $\mathrm{C} 41$ & $0.5102(3)$ & $0.0876(3)$ & $0.76641(19)$ & $0.0157(6)$ & \\
\hline $\mathrm{H} 41$ & 0.560952 & 0.072475 & 0.733666 & $0.019^{*}$ & \\
\hline $\mathrm{C} 42$ & $0.2679(3)$ & $0.1397(3)$ & $0.9042(2)$ & $0.0283(9)$ & \\
\hline $\mathrm{C} 43$ & $0.4979(3)$ & $-0.0924(3)$ & $0.8220(2)$ & $0.0282(8)$ & \\
\hline $\mathrm{C} 44$ & $0.5354(2)$ & $0.3966(3)$ & $0.72353(19)$ & $0.0135(6)$ & \\
\hline $\mathrm{C} 45$ & $0.5700(2)$ & $0.4404(3)$ & $0.8031(2)$ & $0.0145(6)$ & \\
\hline $\mathrm{H} 45$ & 0.580486 & 0.393929 & 0.843987 & $0.017 *$ & \\
\hline $\mathrm{C} 46$ & $0.5893(3)$ & $0.5506(3)$ & $0.8238(2)$ & $0.0160(7)$ & \\
\hline $\mathrm{C} 47$ & $0.5761(3)$ & $0.6217(3)$ & $0.7661(2)$ & $0.0193(7)$ & \\
\hline $\mathrm{H} 47$ & 0.589626 & 0.696700 & 0.780290 & $0.023 *$ & \\
\hline $\mathrm{C} 48$ & $0.5426(3)$ & $0.5799(3)$ & $0.6870(2)$ & $0.0207(7)$ & \\
\hline $\mathrm{C} 49$ & $0.5222(3)$ & 0.4698 & $0.6665(2)$ & $0.0163(7)$ & \\
\hline H49 & 0.498526 & 0.443498 & 0.611851 & $0.020^{*}$ & \\
\hline $\mathrm{C} 50$ & $0.6223(3)$ & $0.5944(3)$ & $0.9098(2)$ & $0.0196(7)$ & \\
\hline $\mathrm{C} 51$ & $0.5298(4)$ & $0.6537(3)$ & $0.6231(2)$ & $0.0335(9)$ & \\
\hline $\mathrm{C} 52$ & $1.1814(3)$ & $0.8865(3)$ & $0.7139(2)$ & $0.0285(8)$ & \\
\hline $\mathrm{C} 53$ & $1.2411(4)$ & $0.9557(4)$ & $0.6583(3)$ & $0.0453(12)$ & \\
\hline H53A & 1.296799 & 0.923935 & 0.640351 & $0.068^{*}$ & \\
\hline H53B & 1.275908 & 1.027343 & 0.685490 & $0.068^{*}$ & \\
\hline $\mathrm{H} 53 \mathrm{C}$ & 1.190809 & 0.962350 & 0.611571 & $0.068^{*}$ & \\
\hline B1 & $0.5245(3)$ & $0.2708(3)$ & $0.6939(2)$ & $0.0129(7)$ & \\
\hline N1 & $1.1302(2)$ & $0.6151(2)$ & $0.78915(17)$ & $0.0189(6)$ & \\
\hline $\mathrm{N} 2$ & $1.1347(2)$ & $0.8327(2)$ & $0.75730(18)$ & $0.0232(6)$ & \\
\hline $\mathrm{F} 1$ & $0.44874(17)$ & $0.04308(16)$ & $0.41230(12)$ & $0.0243(4)$ & \\
\hline $\mathrm{F} 2$ & $0.32971(19)$ & $-0.07221(16)$ & $0.46620(14)$ & $0.0293(5)$ & \\
\hline F3 & $0.2792(2)$ & $0.01634(19)$ & $0.36961(13)$ & $0.0349(6)$ & \\
\hline F19 & $0.6810(2)$ & $0.69749(17)$ & $0.91653(14)$ & $0.0364(6)$ & \\
\hline F20 & 0.53709 (19) & $0.5899(2)$ & $0.94939(14)$ & $0.0409(6)$ & \\
\hline $\mathrm{F} 21$ & $0.68350(18)$ & $0.54092(18)$ & $0.95177(12)$ & $0.0296(5)$ & \\
\hline Rh1 & $1.04312(2)$ & $0.72210(2)$ & $0.82465(2)$ & $0.01406(9)$ & \\
\hline $\mathrm{F} 4$ & $0.1016(4)$ & $0.2763(5)$ & $0.4858(4)$ & $0.0453(7)$ & $0.515(3)$ \\
\hline F5 & $0.1454(7)$ & $0.3337(6)$ & $0.6092(6)$ & $0.0453(7)$ & $0.515(3)$ \\
\hline F6 & 0.0583 (4) & $0.1674(5)$ & $0.5754(4)$ & $0.0453(7)$ & $0.515(3)$ \\
\hline
\end{tabular}




\begin{tabular}{|c|c|c|c|c|c|}
\hline F7 & $1.0095(5)$ & $0.2863(6)$ & $0.7697(3)$ & $0.0323(5)$ & $0.515(3)$ \\
\hline F8 & $0.8820(4)$ & $0.2102(4)$ & $0.8380(3)$ & $0.0323(5)$ & $0.515(3)$ \\
\hline F9 & 0.9217 (4) & $0.1207(5)$ & $0.7392(3)$ & $0.0323(5)$ & $0.515(3)$ \\
\hline F10 & $0.7680(4)$ & $0.4336(7)$ & $0.4766(4)$ & $0.0335(5)$ & $0.515(3)$ \\
\hline F11 & $0.9247(5)$ & $0.4030(4)$ & $0.5062(3)$ & $0.0335(5)$ & $0.515(3)$ \\
\hline F12 & 0.7902 (4) & $0.2806(5)$ & $0.4417(4)$ & $0.0335(5)$ & $0.515(3)$ \\
\hline F13 & $0.271(5)$ & $0.246(3)$ & $0.916(4)$ & 0.028 (4) & $0.515(3)$ \\
\hline F14 & $0.285(3)$ & $0.108(3)$ & $0.9821(19)$ & $0.030(3)$ & $0.515(3)$ \\
\hline F15 & $0.175(2)$ & $0.075(2)$ & $0.8733(19)$ & $0.038(3)$ & $0.515(3)$ \\
\hline F16 & $0.4943(5)$ & $-0.1359(4)$ & $0.8911(5)$ & $0.0354(6)$ & $0.515(3)$ \\
\hline F17 & $0.6008(7)$ & $-0.0794(6)$ & $0.8038(5)$ & $0.0354(6)$ & $0.515(3)$ \\
\hline F18 & $0.4309(5)$ & $-0.1686(5)$ & $0.7668(4)$ & $0.0354(6)$ & $0.515(3)$ \\
\hline F22 & $0.5942(7)$ & $0.7541(7)$ & $0.6449(6)$ & $0.0544(8)$ & $0.515(3)$ \\
\hline F23 & $0.4261(5)$ & $0.6750(4)$ & $0.6230(3)$ & $0.0544(8)$ & $0.515(3)$ \\
\hline F24 & $0.5285(6)$ & $0.6209(4)$ & $0.5520(4)$ & $0.0544(8)$ & $0.515(3)$ \\
\hline F4A & $0.1359(5)$ & $0.3220(5)$ & $0.4955(4)$ & $0.0453(7)$ & $0.485(3)$ \\
\hline F5A & $0.1347(7)$ & $0.3091(7)$ & $0.6223(6)$ & $0.0453(7)$ & $0.485(3)$ \\
\hline F6A & $0.0494(5)$ & $0.1747(6)$ & $0.5369(4)$ & $0.0453(7)$ & $0.485(3)$ \\
\hline F7A & $0.9933(5)$ & $0.2923(6)$ & $0.7922(3)$ & $0.0323(5)$ & $0.485(3)$ \\
\hline F8A & $0.8662(4)$ & $0.1620(5)$ & $0.8244(3)$ & $0.0323(5)$ & $0.485(3)$ \\
\hline F9A & $0.9522(4)$ & $0.1395(5)$ & $0.7234(4)$ & $0.0323(5)$ & $0.485(3)$ \\
\hline F10A & $0.7978(5)$ & $0.4464(7)$ & $0.4864(5)$ & $0.0335(5)$ & 0.485 (3) \\
\hline F11A & $0.9165(5)$ & $0.3575(5)$ & $0.4896(3)$ & $0.0335(5)$ & $0.485(3)$ \\
\hline F12A & $0.7504(5)$ & $0.2806(6)$ & $0.4364(4)$ & $0.0335(5)$ & $0.485(3)$ \\
\hline F13A & $0.260(5)$ & $0.240(4)$ & $0.906(4)$ & $0.037(9)$ & 0.485 (3) \\
\hline F14A & $0.262(3)$ & $0.105(3)$ & $0.973(2)$ & $0.047(8)$ & $0.485(3)$ \\
\hline F15A & $0.165(3)$ & 0.097 (3) & $0.860(2)$ & $0.055(6)$ & $0.485(3)$ \\
\hline F16A & $0.5235(5)$ & $-0.1090(4)$ & $0.9028(5)$ & $0.0354(6)$ & 0.485 (3) \\
\hline F17A & $0.5839(7)$ & $-0.0948(6)$ & $0.7860(5)$ & $0.0354(6)$ & 0.485 (3) \\
\hline F18A & $0.4186(6)$ & $-0.1798(5)$ & $0.7927(4)$ & $0.0354(6)$ & 0.485 (3) \\
\hline F23A & $0.4442(6)$ & $0.6160(5)$ & $0.5702(4)$ & $0.0544(8)$ & $0.485(3)$ \\
\hline F24A & $0.6051(6)$ & $0.6429(5)$ & $0.5650(4)$ & $0.0544(8)$ & $0.485(3)$ \\
\hline F22A & $0.5608(7)$ & $0.7545(8)$ & $0.6407(6)$ & $0.0544(8)$ & $0.485(3)$ \\
\hline
\end{tabular}

\section{Atomic displacement parameters $\left(\AA^{2}\right)$ for (SMK21)}

\begin{tabular}{|l|l|l|l|l|l|l|}
\hline & $U^{11}$ & $U^{22}$ & $U^{33}$ & $U^{12}$ & $U^{13}$ & $U^{23}$ \\
\hline C1 & $0.0282(19)$ & $0.030(2)$ & $0.0236(19)$ & $0.0069(16)$ & $0.0079(16)$ & $-0.0064(16)$ \\
\hline
\end{tabular}




\begin{tabular}{|c|c|c|c|c|c|c|}
\hline $\mathrm{C} 2$ & $0.029(2)$ & $0.041(2)$ & $0.043(2)$ & $0.0177(19)$ & $0.0042(18)$ & $-0.0010(19)$ \\
\hline $\mathrm{C} 3$ & $0.0167(17)$ & $0.0232(18)$ & $0.0279(19)$ & $0.0050(14)$ & $0.0085(15)$ & $0.0058(15)$ \\
\hline $\mathrm{C} 4$ & $0.0159(16)$ & $0.0241(18)$ & $0.0212(18)$ & $0.0025(14)$ & 0.0045 (14) & $0.0071(14)$ \\
\hline $\mathrm{C} 5$ & $0.0202(18)$ & $0.034(2)$ & $0.028(2)$ & $0.0082(15)$ & $0.0028(15)$ & $0.0103(16)$ \\
\hline C6 & $0.0208(18)$ & $0.041(2)$ & $0.0211(19)$ & $0.0011(16)$ & $-0.0025(15)$ & 0.0078 (16) \\
\hline $\mathrm{C} 7$ & $0.0236(18)$ & 0.032 (2) & $0.0174(18)$ & $-0.0039(15)$ & $0.0020(15)$ & $0.0019(15)$ \\
\hline $\mathrm{C} 8$ & $0.0191(17)$ & $0.0236(18)$ & 0.0197 (17) & $0.0006(14)$ & $0.0036(14)$ & $0.0026(14)$ \\
\hline C9 & $0.0140(15)$ & $0.0233(17)$ & $0.0155(16)$ & $0.0010(13)$ & $0.0043(13)$ & $0.0054(13)$ \\
\hline $\mathrm{C} 10$ & $0.0119(15)$ & 0.0205 (17) & $0.0217(18)$ & 0.0029 (13) & $0.0008(13)$ & $-0.0011(14)$ \\
\hline C11 & $0.0172(16)$ & $0.0222(17)$ & $0.0219(18)$ & $0.0071(14)$ & $0.0009(14)$ & $0.0042(14)$ \\
\hline C12 & $0.0149(16)$ & $0.0190(17)$ & $0.0243(18)$ & $0.0061(13)$ & $0.0044(14)$ & $0.0004(14)$ \\
\hline C13 & $0.0122(15)$ & $0.0224(17)$ & $0.0204(17)$ & $0.0047(13)$ & $0.0047(13)$ & $0.0017(14)$ \\
\hline $\mathrm{C} 14$ & $0.0111(15)$ & $0.0171(16)$ & $0.0230(17)$ & $0.0026(12)$ & 0.0045 (13) & $0.0023(13)$ \\
\hline C15 & $0.0214(18)$ & $0.0289(19)$ & $0.0256(19)$ & $0.0027(15)$ & $-0.0012(15)$ & $-0.0073(16)$ \\
\hline $\mathrm{C} 16$ & $0.035(2)$ & 0.034 (2) & $0.028(2)$ & $0.0130(18)$ & $-0.0015(17)$ & 0.0118 (17) \\
\hline C17 & $0.030(2)$ & $0.0201(18)$ & $0.036(2)$ & $0.0094(15)$ & $0.0049(17)$ & $-0.0034(16)$ \\
\hline $\mathrm{C} 18$ & $0.0225(18)$ & $0.034(2)$ & $0.0182(18)$ & $0.0079(15)$ & $0.0056(14)$ & $0.0016(15)$ \\
\hline C19 & $0.0252(19)$ & $0.0186(17)$ & $0.035(2)$ & $0.0062(14)$ & $0.0083(16)$ & $0.0063(15)$ \\
\hline $\mathrm{C} 20$ & $0.0138(15)$ & $0.0116(14)$ & $0.0137(15)$ & $0.0015(12)$ & $0.0032(12)$ & $0.0030(12)$ \\
\hline $\mathrm{C} 21$ & $0.0144(15)$ & $0.0126(15)$ & $0.0166(16)$ & $0.0040(12)$ & $0.0031(13)$ & $0.0031(12)$ \\
\hline $\mathrm{C} 22$ & $0.0187(16)$ & $0.0115(15)$ & $0.0144(16)$ & $0.0037(12)$ & $0.0018(13)$ & $0.0020(12)$ \\
\hline $\mathrm{C} 23$ & $0.0163(16)$ & $0.0165(16)$ & $0.0165(16)$ & $0.0030(13)$ & $-0.0013(13)$ & $-0.0001(13)$ \\
\hline $\mathrm{C} 24$ & $0.0171(16)$ & $0.0213(17)$ & $0.0192(17)$ & $0.0054(13)$ & $0.0028(14)$ & $0.0005(13)$ \\
\hline $\mathrm{C} 25$ & $0.0165(16)$ & $0.0188(16)$ & $0.0159(16)$ & $0.0045(13)$ & $0.0045(13)$ & $-0.0016(13)$ \\
\hline $\mathrm{C} 26$ & $0.0234(17)$ & $0.0154(16)$ & $0.0196(17)$ & $0.0083(13)$ & $-0.0032(14)$ & $-0.0023(13)$ \\
\hline $\mathrm{C} 27$ & $0.0172(18)$ & $0.040(2)$ & $0.026(2)$ & $0.0108(16)$ & $-0.0032(15)$ & $-0.0115(17)$ \\
\hline $\mathrm{C} 28$ & $0.0137(15)$ & $0.0084(14)$ & $0.0145(15)$ & $0.0016(11)$ & $0.0022(12)$ & $-0.0025(11)$ \\
\hline $\mathrm{C} 29$ & $0.0153(15)$ & $0.0135(15)$ & $0.0147(15)$ & $0.0021(12)$ & $0.0030(13)$ & $0.0000(12)$ \\
\hline $\mathrm{C} 30$ & $0.0161(16)$ & $0.0160(16)$ & $0.0185(17)$ & $0.0032(13)$ & $0.0013(13)$ & $-0.0010(13)$ \\
\hline $\mathrm{C} 31$ & $0.0138(15)$ & $0.0186(16)$ & $0.0205(17)$ & $0.0049(13)$ & $0.0042(13)$ & $-0.0007(13)$ \\
\hline C32 & $0.0183(16)$ & $0.0154(15)$ & $0.0171(16)$ & $0.0032(13)$ & $0.0032(13)$ & $-0.0003(13)$ \\
\hline $\mathrm{C} 33$ & $0.0143(15)$ & $0.0136(15)$ & $0.0147(15)$ & $0.0024(12)$ & $0.0019(12)$ & $-0.0005(12)$ \\
\hline C34 & $0.0162(16)$ & $0.0285(19)$ & $0.0215(18)$ & $0.0078(14)$ & $0.0039(14)$ & $0.0043(14)$ \\
\hline C35 & $0.0210(17)$ & 0.0307 (19) & $0.0214(18)$ & $0.0080(15)$ & $0.0077(14)$ & $0.0064(15)$ \\
\hline $\mathrm{C} 36$ & $0.0112(14)$ & $0.0144(15)$ & $0.0125(15)$ & $0.0004(12)$ & $-0.0007(12)$ & $-0.0038(12)$ \\
\hline C37 & $0.0153(15)$ & $0.0127(15)$ & $0.0152(16)$ & $0.0017(12)$ & $0.0009(13)$ & $-0.0047(12)$ \\
\hline C38 & $0.0172(16)$ & $0.0199(17)$ & $0.0157(16)$ & $-0.0007(13)$ & $0.0061(13)$ & $-0.0039(13)$ \\
\hline
\end{tabular}




\begin{tabular}{|c|c|c|c|c|c|c|}
\hline C39 & $0.0245(18)$ & $0.0168(16)$ & $0.0188(17)$ & $-0.0004(14)$ & $0.0083(14)$ & $0.0019(13)$ \\
\hline $\mathrm{C} 40$ & $0.0220(17)$ & $0.0167(16)$ & $0.0203(17)$ & $0.0013(13)$ & $0.0052(14)$ & $0.0008(13)$ \\
\hline $\mathrm{C} 41$ & $0.0162(15)$ & $0.0145(15)$ & $0.0157(16)$ & $0.0028(12)$ & $0.0034(13)$ & $-0.0010(12)$ \\
\hline $\mathrm{C} 42$ & $0.031(2)$ & $0.023(2)$ & $0.031(2)$ & $0.0015(16)$ & $0.0176(18)$ & $-0.0040(16)$ \\
\hline $\mathrm{C} 43$ & $0.034(2)$ & $0.0180(18)$ & $0.036(2)$ & $0.0066(16)$ & $0.0166(17)$ & $0.0091(16)$ \\
\hline C44 & 0.0105 (14) & $0.0149(15)$ & $0.0152(15)$ & $0.0030(12)$ & $0.0034(12)$ & $0.0003(12)$ \\
\hline $\mathrm{C} 45$ & $0.0120(15)$ & $0.0136(15)$ & $0.0181(16)$ & $0.0034(12)$ & $0.0029(12)$ & $0.0017(12)$ \\
\hline $\mathrm{C} 46$ & $0.0122(15)$ & $0.0164(16)$ & 0.0195 (17) & $0.0039(12)$ & $0.0039(13)$ & $-0.0006(13)$ \\
\hline $\mathrm{C} 47$ & $0.0177(16)$ & $0.0126(15)$ & 0.0276 (19) & 0.0047 (13) & 0.0019 (14) & $-0.0005(13)$ \\
\hline C48 & $0.0191(17)$ & $0.0176(17)$ & $0.0250(18)$ & $0.0043(13)$ & $0.0019(14)$ & $0.0044(14)$ \\
\hline C49 & $0.0151(15)$ & $0.0185(16)$ & $0.0140(16)$ & $0.0024(13)$ & $0.0015(13)$ & $0.0004(13)$ \\
\hline C50 & 0.0195 (17) & $0.0156(16)$ & $0.0232(18)$ & 0.0037 (13) & 0.0051 (14) & $-0.0035(13)$ \\
\hline C51 & $0.044(2)$ & $0.0212(19)$ & $0.028(2)$ & $0.0020(17)$ & $-0.0115(18)$ & $0.0059(16)$ \\
\hline C52 & $0.031(2)$ & $0.0249(19)$ & 0.0235 (19) & $-0.0023(16)$ & $0.0023(17)$ & $0.0026(16)$ \\
\hline C53 & $0.054(3)$ & $0.040(3)$ & $0.028(2)$ & $-0.013(2)$ & $0.008(2)$ & $0.0095(19)$ \\
\hline B1 & $0.0123(16)$ & $0.0132(17)$ & 0.0135 (17) & $0.0038(13)$ & $0.0025(14)$ & $-0.0006(13)$ \\
\hline N1 & 0.0165 (14) & $0.0204(14)$ & $0.0193(15)$ & $0.0028(11)$ & $0.0056(12)$ & $0.0001(11)$ \\
\hline $\mathrm{N} 2$ & $0.0228(15)$ & $0.0242(15)$ & $0.0200(15)$ & $0.0022(13)$ & 0.0017 (13) & 0.0025 (13) \\
\hline $\mathrm{F} 1$ & $0.0316(11)$ & $0.0224(10)$ & $0.0219(10)$ & $0.0114(9)$ & $0.0081(9)$ & $-0.0032(8)$ \\
\hline F2 & $0.0388(13)$ & $0.0108(9)$ & $0.0380(13)$ & $0.0057(9)$ & $0.0070(10)$ & $-0.0006(9)$ \\
\hline F3 & $0.0428(13)$ & 0.0357 (13) & $0.0262(12)$ & $0.0223(11)$ & $-0.0162(10)$ & $-0.0171(10)$ \\
\hline F19 & $0.0522(15)$ & $0.0183(11)$ & $0.0299(12)$ & $-0.0019(10)$ & $-0.0006(11)$ & $-0.0080(9)$ \\
\hline F20 & $0.0255(12)$ & $0.0696(18)$ & $0.0273(12)$ & $0.0121(12)$ & $0.0099(10)$ & $-0.0118(12)$ \\
\hline F21 & $0.0372(12)$ & $0.0312(12)$ & $0.0202(10)$ & $0.0145(10)$ & $-0.0070(9)$ & $-0.0061(9)$ \\
\hline Rh1 & $0.01340(13)$ & 0.01479 (13) & $0.01323(14)$ & $0.00262(9)$ & $0.00138(9)$ & $0.00076(9)$ \\
\hline F4 & $0.0247(11)$ & $0.0586(18)$ & $0.0547(16)$ & $0.0210(12)$ & $-0.0027(12)$ & $-0.0138(15)$ \\
\hline F5 & $0.0247(11)$ & $0.0586(18)$ & 0.0547 (16) & $0.0210(12)$ & $-0.0027(12)$ & $-0.0138(15)$ \\
\hline F6 & $0.0247(11)$ & $0.0586(18)$ & $0.0547(16)$ & $0.0210(12)$ & $-0.0027(12)$ & $-0.0138(15)$ \\
\hline F7 & $0.0258(11)$ & $0.0415(12)$ & $0.0326(14)$ & $0.0148(11)$ & $0.0008(9)$ & $0.0064(11)$ \\
\hline F8 & $0.0258(11)$ & $0.0415(12)$ & $0.0326(14)$ & $0.0148(11)$ & $0.0008(9)$ & $0.0064(11)$ \\
\hline F9 & $0.0258(11)$ & $0.0415(12)$ & $0.0326(14)$ & $0.0148(11)$ & $0.0008(9)$ & $0.0064(11)$ \\
\hline F10 & $0.0335(15)$ & $0.0408(12)$ & $0.0265(11)$ & $0.0066(17)$ & $0.0103(12)$ & $0.0082(10)$ \\
\hline F11 & $0.0335(15)$ & $0.0408(12)$ & $0.0265(11)$ & $0.0066(17)$ & $0.0103(12)$ & $0.0082(10)$ \\
\hline F12 & $0.0335(15)$ & $0.0408(12)$ & $0.0265(11)$ & $0.0066(17)$ & $0.0103(12)$ & $0.0082(10)$ \\
\hline F13 & $0.040(10)$ & $0.023(7)$ & $0.028(7)$ & $0.015(5)$ & $0.019(6)$ & $-0.001(4)$ \\
\hline F14 & $0.047(9)$ & $0.029(5)$ & $0.022(4)$ & $0.014(5)$ & $0.023(5)$ & $0.002(3)$ \\
\hline F15 & $0.015(6)$ & $0.041(7)$ & $0.054(8)$ & $0.001(4)$ & $0.015(5)$ & $-0.011(4)$ \\
\hline
\end{tabular}




\begin{tabular}{|l|l|l|l|l|l|l|}
\hline F16 & $0.0466(15)$ & $0.0165(12)$ & $0.046(2)$ & $0.0083(11)$ & $0.0162(13)$ & $0.0090(11)$ \\
\hline F17 & $0.0466(15)$ & $0.0165(12)$ & $0.046(2)$ & $0.0083(11)$ & $0.0162(13)$ & $0.0090(11)$ \\
\hline F18 & $0.0466(15)$ & $0.0165(12)$ & $0.046(2)$ & $0.0083(11)$ & $0.0162(13)$ & $0.0090(11)$ \\
\hline F22 & $0.084(2)$ & $0.0374(11)$ & $0.0442(12)$ & $0.0220(17)$ & $-0.0044(17)$ & $0.0212(9)$ \\
\hline F23 & $0.084(2)$ & $0.0374(11)$ & $0.0442(12)$ & $0.0220(17)$ & $-0.0044(17)$ & $0.0212(9)$ \\
\hline F24 & $0.084(2)$ & $0.0374(11)$ & $0.0442(12)$ & $0.0220(17)$ & $-0.0044(17)$ & $0.0212(9)$ \\
\hline F4A & $0.0247(11)$ & $0.0586(18)$ & $0.0547(16)$ & $0.0210(12)$ & $-0.0027(12)$ & $-0.0138(15)$ \\
\hline F5A & $0.0247(11)$ & $0.0586(18)$ & $0.0547(16)$ & $0.0210(12)$ & $-0.0027(12)$ & $-0.0138(15)$ \\
\hline F6A & $0.0247(11)$ & $0.0586(18)$ & $0.0547(16)$ & $0.0210(12)$ & $-0.0027(12)$ & $-0.0138(15)$ \\
\hline F7A & $0.0258(11)$ & $0.0415(12)$ & $0.0326(14)$ & $0.0148(11)$ & $0.0008(9)$ & $0.0064(11)$ \\
\hline F8A & $0.0258(11)$ & $0.0415(12)$ & $0.0326(14)$ & $0.0148(11)$ & $0.0008(9)$ & $0.0064(11)$ \\
\hline F9A & $0.0258(11)$ & $0.0415(12)$ & $0.0326(14)$ & $0.0148(11)$ & $0.0008(9)$ & $0.0064(11)$ \\
\hline F10A & $0.0335(15)$ & $0.0408(12)$ & $0.0265(11)$ & $0.0066(17)$ & $0.0103(12)$ & $0.0082(10)$ \\
\hline F11A & $0.0335(15)$ & $0.0408(12)$ & $0.0265(11)$ & $0.0066(17)$ & $0.0103(12)$ & $0.0082(10)$ \\
\hline F12A & $0.0335(15)$ & $0.0408(12)$ & $0.0265(11)$ & $0.0066(17)$ & $0.0103(12)$ & $0.0082(10)$ \\
\hline F13A & $0.039(10)$ & $0.040(11)$ & $0.040(18)$ & $0.015(7)$ & $0.026(13)$ & $0.003(9)$ \\
\hline F14A & $0.067(18)$ & $0.040(6)$ & $0.046(13)$ & $0.017(9)$ & $0.045(12)$ & $0.015(8)$ \\
\hline F15A & $0.019(5)$ & $0.070(15)$ & $0.071(14)$ & $0.006(8)$ & $0.011(7)$ & $-0.026(9)$ \\
\hline F16A & $0.0466(15)$ & $0.0165(12)$ & $0.046(2)$ & $0.0083(11)$ & $0.0162(13)$ & $0.0090(11)$ \\
\hline F17A & $0.0466(15)$ & $0.0165(12)$ & $0.046(2)$ & $0.0083(11)$ & $0.0162(13)$ & $0.0090(11)$ \\
\hline F18A & $0.0466(15)$ & $0.0165(12)$ & $0.046(2)$ & $0.0083(11)$ & $0.0162(13)$ & $0.0090(11)$ \\
\hline F23A & $0.084(2)$ & $0.0374(11)$ & $0.0442(12)$ & $0.0220(17)$ & $-0.0044(17)$ & $0.0212(9)$ \\
\hline F24A & $0.084(2)$ & $0.0374(11)$ & $0.0442(12)$ & $0.0220(17)$ & $-0.0044(17)$ & $0.0212(9)$ \\
\hline F22A & $0.084(2)$ & $0.0374(11)$ & $0.0442(12)$ & $0.0220(17)$ & $-0.0044(17)$ & $0.0212(9)$ \\
\hline
\end{tabular}

\section{Geometric parameters $\left(\AA,{ }^{\circ}\right)$ for (SMK21)}

\begin{tabular}{|l|l|l|l|}
\hline $\mathrm{C} 1-\mathrm{N} 1$ & $1.465(5)$ & $\mathrm{C} 28-\mathrm{B} 1$ & $1.643(4)$ \\
\hline $\mathrm{C} 1-\mathrm{H} 1 \mathrm{~A}$ & 0.9800 & $\mathrm{C} 29-\mathrm{C} 30$ & $1.393(5)$ \\
\hline $\mathrm{C} 1-\mathrm{H} 1 \mathrm{~B}$ & 0.9800 & $\mathrm{C} 29-\mathrm{H} 29$ & 0.9500 \\
\hline $\mathrm{C} 1-\mathrm{H} 1 \mathrm{C}$ & 0.9800 & $\mathrm{C} 30-\mathrm{C} 31$ & $1.385(5)$ \\
\hline $\mathrm{C} 2-\mathrm{C} 3$ & $1.500(5)$ & $\mathrm{C} 30-\mathrm{C} 34$ & $1.499(5)$ \\
\hline $\mathrm{C} 2-\mathrm{H} 2 \mathrm{~A}$ & 0.9800 & $\mathrm{C} 31-\mathrm{C} 32$ & $1.391(5)$ \\
\hline $\mathrm{C} 2-\mathrm{H} 2 \mathrm{~B}$ & 0.9800 & $\mathrm{C} 31-\mathrm{H} 31$ & 0.9500 \\
\hline $\mathrm{C} 2-\mathrm{H} 2 \mathrm{C}$ & 0.9800 & $\mathrm{C} 32-\mathrm{C} 33$ & $1.394(5)$ \\
\hline $\mathrm{C} 3-\mathrm{N} 1$ & $1.292(5)$ & $\mathrm{C} 32-\mathrm{C} 35$ & $1.494(5)$ \\
\hline $\mathrm{C} 3-\mathrm{C} 4$ & $1.468(5)$ & $\mathrm{C} 33-\mathrm{H} 33$ & 0.9500 \\
\hline $\mathrm{C} 4-\mathrm{C} 5$ & $1.397(5)$ & $\mathrm{C} 34-\mathrm{F} 9$ & $1.295(7)$ \\
\hline
\end{tabular}




\begin{tabular}{|c|c|c|c|}
\hline $\mathrm{C} 4-\mathrm{C} 9$ & $1.411(5)$ & C34-F7A & $1.317(8)$ \\
\hline $\mathrm{C} 5-\mathrm{C} 6$ & $1.382(6)$ & $\mathrm{C} 34-\mathrm{F} 8 \mathrm{~A}$ & $1.347(7)$ \\
\hline $\mathrm{C} 5-\mathrm{H} 5$ & 0.9500 & C34-F8 & $1.351(6)$ \\
\hline $\mathrm{C} 6-\mathrm{C} 7$ & $1.388(6)$ & C34-F7 & $1.381(7)$ \\
\hline C6- $-\mathrm{H} 6$ & 0.9500 & C34-F9A & $1.396(7)$ \\
\hline $\mathrm{C} 7-\mathrm{C} 8$ & $1.395(5)$ & $\mathrm{C} 35-\mathrm{F} 12$ & $1.277(8)$ \\
\hline $\mathrm{C} 7-\mathrm{H} 7$ & 0.9500 & $\mathrm{C} 35-\mathrm{F} 10 \mathrm{~A}$ & $1.300(10)$ \\
\hline $\mathrm{C} 8-\mathrm{C} 9$ & $1.387(5)$ & C35-F11A & $1.328(7)$ \\
\hline $\mathrm{C} 8-\mathrm{H} 8$ & 0.9500 & $\mathrm{C} 35-\mathrm{F} 10$ & $1.377(9)$ \\
\hline C9-Rh1 & $2.031(3)$ & C35-F11 & $1.382(7)$ \\
\hline $\mathrm{C} 10-\mathrm{C} 11$ & $1.400(5)$ & C35-F12A & $1.419(8)$ \\
\hline $\mathrm{C} 10-\mathrm{C} 14$ & $1.457(5)$ & C36-C37 & $1.403(5)$ \\
\hline $\mathrm{C} 10-\mathrm{C} 15$ & $1.498(5)$ & $\mathrm{C} 36-\mathrm{C} 41$ & $1.403(5)$ \\
\hline C10-Rh1 & $2.255(3)$ & $\mathrm{C} 36-\mathrm{B} 1$ & $1.637(5)$ \\
\hline $\mathrm{C} 11-\mathrm{C} 12$ & $1.452(5)$ & C37-C38 & $1.390(5)$ \\
\hline $\mathrm{C} 11-\mathrm{C} 16$ & $1.499(5)$ & C37-H37 & 0.9500 \\
\hline $\mathrm{C} 11-\mathrm{Rh} 1$ & $2.243(3)$ & C38-C39 & $1.389(5)$ \\
\hline $\mathrm{C} 12-\mathrm{C} 13$ & $1.427(5)$ & $\mathrm{C} 38-\mathrm{C} 42$ & $1.499(5)$ \\
\hline $\mathrm{C} 12-\mathrm{C} 17$ & $1.495(5)$ & $\mathrm{C} 39-\mathrm{C} 40$ & $1.389(5)$ \\
\hline $\mathrm{C} 12-\mathrm{Rh} 1$ & $2.171(3)$ & С39-H39 & 0.9500 \\
\hline $\mathrm{C} 13-\mathrm{C} 14$ & $1.431(5)$ & $\mathrm{C} 40-\mathrm{C} 41$ & $1.392(5)$ \\
\hline $\mathrm{C} 13-\mathrm{C} 18$ & $1.498(5)$ & $\mathrm{C} 40-\mathrm{C} 43$ & $1.502(5)$ \\
\hline C13-Rh1 & 2.177 (3) & $\mathrm{C} 41-\mathrm{H} 41$ & 0.9500 \\
\hline $\mathrm{C} 14-\mathrm{C} 19$ & $1.500(5)$ & C42-F14A & $1.26(4)$ \\
\hline C14-Rh1 & $2.142(3)$ & $\mathrm{C} 42-\mathrm{F} 15$ & $1.30(3)$ \\
\hline $\mathrm{C} 15-\mathrm{H} 15 \mathrm{~A}$ & 0.9800 & $\mathrm{C} 42-\mathrm{F} 13 \mathrm{~A}$ & $1.32(5)$ \\
\hline C15-H15B & 0.9800 & $\mathrm{C} 42-\mathrm{F} 13$ & $1.36(4)$ \\
\hline $\mathrm{C} 15-\mathrm{H} 15 \mathrm{C}$ & 0.9800 & $\mathrm{C} 42-\mathrm{F} 14$ & $1.40(4)$ \\
\hline C16-H16A & 0.9800 & $\mathrm{C} 42-\mathrm{F} 15 \mathrm{~A}$ & $1.41(4)$ \\
\hline C16-H16B & 0.9800 & $\mathrm{C} 43-\mathrm{F} 16$ & $1.308(9)$ \\
\hline $\mathrm{C} 16-\mathrm{H} 16 \mathrm{C}$ & 0.9800 & $\mathrm{C} 43-\mathrm{F} 17 \mathrm{~A}$ & $1.325(11)$ \\
\hline C17-H17A & 0.9800 & C43-F18A & $1.337(9)$ \\
\hline C17-H17B & 0.9800 & C43-F17 & $1.361(10)$ \\
\hline C17-H17C & 0.9800 & $\mathrm{C} 43-\mathrm{F} 18$ & $1.365(8)$ \\
\hline $\mathrm{C} 18-\mathrm{H} 18 \mathrm{~A}$ & 0.9800 & C43-F16A & $1.396(9)$ \\
\hline $\mathrm{C} 18-\mathrm{H} 18 \mathrm{~B}$ & 0.9800 & $\mathrm{C} 44-\mathrm{C} 49$ & $1.399(5)$ \\
\hline $\mathrm{C} 18-\mathrm{H} 18 \mathrm{C}$ & 0.9800 & $\mathrm{C} 44-\mathrm{C} 45$ & $1.403(5)$ \\
\hline
\end{tabular}




\begin{tabular}{|c|c|c|c|}
\hline C19-H19A & 0.9800 & $\mathrm{C} 44-\mathrm{B} 1$ & $1.639(4)$ \\
\hline C19-H19B & 0.9800 & $\mathrm{C} 45-\mathrm{C} 46$ & $1.395(5)$ \\
\hline $\mathrm{C} 19-\mathrm{H} 19 \mathrm{C}$ & 0.9800 & $\mathrm{C} 45-\mathrm{H} 45$ & 0.9500 \\
\hline $\mathrm{C} 20-\mathrm{C} 21$ & $1.395(4)$ & $\mathrm{C} 46-\mathrm{C} 47$ & $1.387(5)$ \\
\hline $\mathrm{C} 20-\mathrm{C} 25$ & $1.402(5)$ & $\mathrm{C} 46-\mathrm{C} 50$ & $1.498(5)$ \\
\hline $\mathrm{C} 20-\mathrm{B} 1$ & $1.649(5)$ & $\mathrm{C} 47-\mathrm{C} 48$ & $1.386(5)$ \\
\hline $\mathrm{C} 21-\mathrm{C} 22$ & $1.396(5)$ & $\mathrm{C} 47-\mathrm{H} 47$ & 0.9500 \\
\hline $\mathrm{C} 21-\mathrm{H} 21$ & 0.9500 & $\mathrm{C} 48-\mathrm{C} 49$ & $1.392(5)$ \\
\hline $\mathrm{C} 22-\mathrm{C} 23$ & $1.378(5)$ & C48-C51 & $1.485(5)$ \\
\hline $\mathrm{C} 22-\mathrm{C} 26$ & $1.496(4)$ & $\mathrm{C} 49-\mathrm{H} 49$ & 0.9500 \\
\hline $\mathrm{C} 23-\mathrm{C} 24$ & $1.394(5)$ & $\mathrm{C} 50-\mathrm{F} 20$ & $1.334(4)$ \\
\hline $\mathrm{C} 23-\mathrm{H} 23$ & 0.9500 & $\mathrm{C} 50-\mathrm{F} 19$ & $1.337(4)$ \\
\hline $\mathrm{C} 24-\mathrm{C} 25$ & $1.390(5)$ & $\mathrm{C} 50-\mathrm{F} 21$ & $1.338(4)$ \\
\hline $\mathrm{C} 24-\mathrm{C} 27$ & $1.492(5)$ & C51-F24 & $1.244(7)$ \\
\hline $\mathrm{C} 25-\mathrm{H} 25$ & 0.9500 & $\mathrm{C} 51-\mathrm{F} 22 \mathrm{~A}$ & $1.265(11)$ \\
\hline $\mathrm{C} 26-\mathrm{F} 1$ & $1.339(4)$ & $\mathrm{C} 51-\mathrm{F} 23 \mathrm{~A}$ & $1.303(8)$ \\
\hline $\mathrm{C} 26-\mathrm{F} 3$ & $1.341(4)$ & $\mathrm{C} 51-\mathrm{F} 22$ & $1.349(10)$ \\
\hline $\mathrm{C} 26-\mathrm{F} 2$ & $1.350(4)$ & C51-F23 & $1.430(8)$ \\
\hline $\mathrm{C} 27-\mathrm{F} 6 \mathrm{~A}$ & $1.310(8)$ & $\mathrm{C} 51-\mathrm{F} 24 \mathrm{~A}$ & $1.475(9)$ \\
\hline $\mathrm{C} 27-\mathrm{F} 4$ & $1.320(8)$ & $\mathrm{C} 52-\mathrm{N} 2$ & $1.137(5)$ \\
\hline $\mathrm{C} 27-\mathrm{F} 5 \mathrm{~A}$ & $1.318(11)$ & $\mathrm{C} 52-\mathrm{C} 53$ & $1.459(6)$ \\
\hline $\mathrm{C} 27-\mathrm{F} 5$ & $1.331(10)$ & $\mathrm{C} 53-\mathrm{H} 53 \mathrm{~A}$ & 0.9800 \\
\hline $\mathrm{C} 27-\mathrm{F} 4 \mathrm{~A}$ & $1.407(8)$ & $\mathrm{C} 53-\mathrm{H} 53 \mathrm{~B}$ & 0.9800 \\
\hline $\mathrm{C} 27-\mathrm{F} 6$ & $1.368(8)$ & C $53-\mathrm{H} 53 \mathrm{C}$ & 0.9800 \\
\hline $\mathrm{C} 28-\mathrm{C} 33$ & $1.400(5)$ & $\mathrm{N} 1-\mathrm{Rh} 1$ & 2.095 (3) \\
\hline $\mathrm{C} 28-\mathrm{C} 29$ & $1.405(5)$ & $\mathrm{N} 2-\mathrm{Rh} 1$ & $2.061(3)$ \\
\hline $\mathrm{N} 1-\mathrm{C} 1-\mathrm{H} 1 \mathrm{~A}$ & 109.5 & F7A $-\mathrm{C} 34-\mathrm{F} 8 \mathrm{~A}$ & $107.6(4)$ \\
\hline $\mathrm{N} 1-\mathrm{C} 1-\mathrm{H} 1 \mathrm{~B}$ & 109.5 & F9-C34-F8 & $109.4(4)$ \\
\hline $\mathrm{H} 1 \mathrm{~A}-\mathrm{C} 1-\mathrm{H} 1 \mathrm{~B}$ & 109.5 & F9-C34-F7 & $106.4(4)$ \\
\hline $\mathrm{N} 1-\mathrm{C} 1-\mathrm{H} 1 \mathrm{C}$ & 109.5 & $\mathrm{~F} 8-\mathrm{C} 34-\mathrm{F} 7$ & $104.1(4)$ \\
\hline $\mathrm{H} 1 \mathrm{~A}-\mathrm{C} 1-\mathrm{H} 1 \mathrm{C}$ & 109.5 & F7A $-\mathrm{C} 34-\mathrm{F} 9 \mathrm{~A}$ & $104.4(4)$ \\
\hline $\mathrm{H} 1 \mathrm{~B}-\mathrm{C} 1-\mathrm{H} 1 \mathrm{C}$ & 109.5 & $\mathrm{~F} 8 \mathrm{~A}-\mathrm{C} 34-\mathrm{F} 9 \mathrm{~A}$ & $104.8(4)$ \\
\hline $\mathrm{C} 3-\mathrm{C} 2-\mathrm{H} 2 \mathrm{~A}$ & 109.5 & $\mathrm{~F} 9-\mathrm{C} 34-\mathrm{C} 30$ & $113.2(4)$ \\
\hline $\mathrm{C} 3-\mathrm{C} 2-\mathrm{H} 2 \mathrm{~B}$ & 109.5 & F7A-C34-C30 & $113.6(4)$ \\
\hline $\mathrm{H} 2 \mathrm{~A}-\mathrm{C} 2-\mathrm{H} 2 \mathrm{~B}$ & 109.5 & $\mathrm{~F} 8 \mathrm{~A}-\mathrm{C} 34-\mathrm{C} 30$ & $114.2(3)$ \\
\hline $\mathrm{C} 3-\mathrm{C} 2-\mathrm{H} 2 \mathrm{C}$ & 109.5 & $\mathrm{~F} 8-\mathrm{C} 34-\mathrm{C} 30$ & $112.0(3)$ \\
\hline
\end{tabular}




\begin{tabular}{|c|c|c|c|}
\hline $\mathrm{H} 2 \mathrm{~A}-\mathrm{C} 2-\mathrm{H} 2 \mathrm{C}$ & 109.5 & $\mathrm{~F} 7-\mathrm{C} 34-\mathrm{C} 30$ & $111.2(4)$ \\
\hline $\mathrm{H} 2 \mathrm{~B}-\mathrm{C} 2-\mathrm{H} 2 \mathrm{C}$ & 109.5 & F9A-C34-C30 & $111.4(4)$ \\
\hline $\mathrm{N} 1-\mathrm{C} 3-\mathrm{C} 4$ & $114.7(3)$ & $\mathrm{F} 10 \mathrm{~A}-\mathrm{C} 35-\mathrm{F} 11 \mathrm{~A}$ & $108.4(4)$ \\
\hline $\mathrm{N} 1-\mathrm{C} 3-\mathrm{C} 2$ & $123.4(3)$ & $\mathrm{F} 12-\mathrm{C} 35-\mathrm{F} 10$ & $106.6(5)$ \\
\hline $\mathrm{C} 4-\mathrm{C} 3-\mathrm{C} 2$ & $121.8(3)$ & $\mathrm{F} 12-\mathrm{C} 35-\mathrm{F} 11$ & $108.4(4)$ \\
\hline $\mathrm{C} 5-\mathrm{C} 4-\mathrm{C} 9$ & $120.5(3)$ & $\mathrm{F} 10-\mathrm{C} 35-\mathrm{F} 11$ & $103.2(4)$ \\
\hline $\mathrm{C} 5-\mathrm{C} 4-\mathrm{C} 3$ & $124.5(3)$ & $\mathrm{F} 10 \mathrm{~A}-\mathrm{C} 35-\mathrm{F} 12 \mathrm{~A}$ & $105.4(5)$ \\
\hline $\mathrm{C} 9-\mathrm{C} 4-\mathrm{C} 3$ & $114.9(3)$ & $\mathrm{F} 11 \mathrm{~A}-\mathrm{C} 35-\mathrm{F} 12 \mathrm{~A}$ & $104.7(4)$ \\
\hline $\mathrm{C} 6-\mathrm{C} 5-\mathrm{C} 4$ & $119.9(4)$ & $\mathrm{F} 12-\mathrm{C} 35-\mathrm{C} 32$ & $115.1(4)$ \\
\hline $\mathrm{C} 6-\mathrm{C} 5-\mathrm{H} 5$ & 120.1 & $\mathrm{~F} 10 \mathrm{~A}-\mathrm{C} 35-\mathrm{C} 32$ & $113.3(5)$ \\
\hline $\mathrm{C} 4-\mathrm{C} 5-\mathrm{H} 5$ & 120.1 & $\mathrm{~F} 11 \mathrm{~A}-\mathrm{C} 35-\mathrm{C} 32$ & $114.5(4)$ \\
\hline $\mathrm{C} 5-\mathrm{C} 6-\mathrm{C} 7$ & $120.0(3)$ & F10-C35-C32 & $111.7(4)$ \\
\hline $\mathrm{C} 5-\mathrm{C} 6-\mathrm{H} 6$ & 120.0 & F11-C35-C32 & $111.1(3)$ \\
\hline $\mathrm{C} 7-\mathrm{C} 6-\mathrm{H} 6$ & 120.0 & $\mathrm{~F} 12 \mathrm{~A}-\mathrm{C} 35-\mathrm{C} 32$ & $109.7(4)$ \\
\hline $\mathrm{C} 6-\mathrm{C} 7-\mathrm{C} 8$ & $120.4(4)$ & $\mathrm{C} 37-\mathrm{C} 36-\mathrm{C} 41$ & $115.7(3)$ \\
\hline $\mathrm{C} 6-\mathrm{C} 7-\mathrm{H} 7$ & 119.8 & $\mathrm{C} 37-\mathrm{C} 36-\mathrm{B} 1$ & $120.8(3)$ \\
\hline $\mathrm{C} 8-\mathrm{C} 7-\mathrm{H} 7$ & 119.8 & $\mathrm{C} 41-\mathrm{C} 36-\mathrm{B} 1$ & $122.9(3)$ \\
\hline $\mathrm{C} 9-\mathrm{C} 8-\mathrm{C} 7$ & $120.6(3)$ & $\mathrm{C} 38-\mathrm{C} 37-\mathrm{C} 36$ & $122.2(3)$ \\
\hline $\mathrm{C} 9-\mathrm{C} 8-\mathrm{H} 8$ & 119.7 & C38-C37-H37 & 118.9 \\
\hline $\mathrm{C} 7-\mathrm{C} 8-\mathrm{H} 8$ & 119.7 & $\mathrm{C} 36-\mathrm{C} 37-\mathrm{H} 37$ & 118.9 \\
\hline $\mathrm{C} 8-\mathrm{C} 9-\mathrm{C} 4$ & $118.7(3)$ & $\mathrm{C} 39-\mathrm{C} 38-\mathrm{C} 37$ & $121.1(3)$ \\
\hline $\mathrm{C} 8-\mathrm{C} 9-\mathrm{Rh} 1$ & $126.9(3)$ & $\mathrm{C} 39-\mathrm{C} 38-\mathrm{C} 42$ & $118.8(3)$ \\
\hline $\mathrm{C} 4-\mathrm{C} 9-\mathrm{Rh} 1$ & $114.4(2)$ & $\mathrm{C} 37-\mathrm{C} 38-\mathrm{C} 42$ & $120.1(3)$ \\
\hline $\mathrm{C} 11-\mathrm{C} 10-\mathrm{C} 14$ & $107.1(3)$ & $\mathrm{C} 40-\mathrm{C} 39-\mathrm{C} 38$ & $117.8(3)$ \\
\hline $\mathrm{C} 11-\mathrm{C} 10-\mathrm{C} 15$ & $127.7(3)$ & $\mathrm{C} 40-\mathrm{C} 39-\mathrm{H} 39$ & 121.1 \\
\hline $\mathrm{C} 14-\mathrm{C} 10-\mathrm{C} 15$ & $125.2(3)$ & C $38-\mathrm{C} 39-\mathrm{H} 39$ & 121.1 \\
\hline $\mathrm{C} 11-\mathrm{C} 10-\mathrm{Rh} 1$ & $71.43(19)$ & $\mathrm{C} 39-\mathrm{C} 40-\mathrm{C} 41$ & $121.0(3)$ \\
\hline $\mathrm{C} 14-\mathrm{C} 10-\mathrm{Rh} 1$ & $66.51(18)$ & $\mathrm{C} 39-\mathrm{C} 40-\mathrm{C} 43$ & $118.9(3)$ \\
\hline $\mathrm{C} 15-\mathrm{C} 10-\mathrm{Rh} 1$ & $129.1(2)$ & $\mathrm{C} 41-\mathrm{C} 40-\mathrm{C} 43$ & $120.0(3)$ \\
\hline $\mathrm{C} 10-\mathrm{C} 11-\mathrm{C} 12$ & $109.3(3)$ & $\mathrm{C} 40-\mathrm{C} 41-\mathrm{C} 36$ & $122.2(3)$ \\
\hline $\mathrm{C} 10-\mathrm{C} 11-\mathrm{C} 16$ & $126.6(3)$ & $\mathrm{C} 40-\mathrm{C} 41-\mathrm{H} 41$ & 118.9 \\
\hline $\mathrm{C} 12-\mathrm{C} 11-\mathrm{C} 16$ & $124.1(3)$ & $\mathrm{C} 36-\mathrm{C} 41-\mathrm{H} 41$ & 118.9 \\
\hline $\mathrm{C} 10-\mathrm{C} 11-\mathrm{Rh} 1$ & $72.31(19)$ & $\mathrm{F} 14 \mathrm{~A}-\mathrm{C} 42-\mathrm{F} 13 \mathrm{~A}$ & $110(3)$ \\
\hline $\mathrm{C} 12-\mathrm{C} 11-\mathrm{Rh} 1$ & $68.13(18)$ & $\mathrm{F} 15-\mathrm{C} 42-\mathrm{F} 13$ & $116(3)$ \\
\hline $\mathrm{C} 16-\mathrm{C} 11-\mathrm{Rh} 1$ & $126.3(3)$ & $\mathrm{F} 15-\mathrm{C} 42-\mathrm{F} 14$ & $104.3(19)$ \\
\hline $\mathrm{C} 13-\mathrm{C} 12-\mathrm{C} 11$ & $107.3(3)$ & $\mathrm{F} 13-\mathrm{C} 42-\mathrm{F} 14$ & $104(3)$ \\
\hline $\mathrm{C} 13-\mathrm{C} 12-\mathrm{C} 17$ & $127.1(3)$ & $\mathrm{F} 14 \mathrm{~A}-\mathrm{C} 42-\mathrm{F} 15 \mathrm{~A}$ & $107(2)$ \\
\hline
\end{tabular}




\begin{tabular}{|c|c|c|c|}
\hline $\mathrm{C} 11-\mathrm{C} 12-\mathrm{C} 17$ & $124.7(3)$ & $\mathrm{F} 13 \mathrm{~A}-\mathrm{C} 42-\mathrm{F} 15 \mathrm{~A}$ & $94(3)$ \\
\hline $\mathrm{C} 13-\mathrm{C} 12-\mathrm{Rh} 1$ & 71.09 (19) & $\mathrm{F} 14 \mathrm{~A}-\mathrm{C} 42-\mathrm{C} 38$ & $118.8(17)$ \\
\hline $\mathrm{C} 11-\mathrm{C} 12-\mathrm{Rh} 1$ & $73.50(19)$ & $\mathrm{F} 15-\mathrm{C} 42-\mathrm{C} 38$ & $110.6(14)$ \\
\hline $\mathrm{C} 17-\mathrm{C} 12-\mathrm{Rh} 1$ & $128.8(2)$ & $\mathrm{F} 13 \mathrm{~A}-\mathrm{C} 42-\mathrm{C} 38$ & $113(2)$ \\
\hline $\mathrm{C} 12-\mathrm{C} 13-\mathrm{C} 14$ & $107.7(3)$ & $\mathrm{F} 13-\mathrm{C} 42-\mathrm{C} 38$ & $112(2)$ \\
\hline $\mathrm{C} 12-\mathrm{C} 13-\mathrm{C} 18$ & $126.9(3)$ & $\mathrm{F} 14-\mathrm{C} 42-\mathrm{C} 38$ & $109.0(14)$ \\
\hline $\mathrm{C} 14-\mathrm{C} 13-\mathrm{C} 18$ & $125.4(3)$ & $\mathrm{F} 15 \mathrm{~A}-\mathrm{C} 42-\mathrm{C} 38$ & $111.9(16)$ \\
\hline $\mathrm{C} 12-\mathrm{C} 13-\mathrm{Rh} 1$ & $70.61(19)$ & $\mathrm{F} 17 \mathrm{~A}-\mathrm{C} 43-\mathrm{F} 18 \mathrm{~A}$ & $107.0(4)$ \\
\hline $\mathrm{C} 14-\mathrm{C} 13-\mathrm{Rh} 1$ & $69.30(19)$ & $\mathrm{F} 16-\mathrm{C} 43-\mathrm{F} 17$ & $106.4(5)$ \\
\hline $\mathrm{C} 18-\mathrm{C} 13-\mathrm{Rh} 1$ & $127.2(2)$ & $\mathrm{F} 16-\mathrm{C} 43-\mathrm{F} 18$ & $105.8(4)$ \\
\hline $\mathrm{C} 13-\mathrm{C} 14-\mathrm{C} 10$ & $108.3(3)$ & $\mathrm{F} 17-\mathrm{C} 43-\mathrm{F} 18$ & $106.9(4)$ \\
\hline $\mathrm{C} 13-\mathrm{C} 14-\mathrm{C} 19$ & $125.8(3)$ & $\mathrm{F} 17 \mathrm{~A}-\mathrm{C} 43-\mathrm{F} 16 \mathrm{~A}$ & $106.7(6)$ \\
\hline $\mathrm{C} 10-\mathrm{C} 14-\mathrm{C} 19$ & $125.5(3)$ & $\mathrm{F} 18 \mathrm{~A}-\mathrm{C} 43-\mathrm{F} 16 \mathrm{~A}$ & $105.1(4)$ \\
\hline $\mathrm{C} 13-\mathrm{C} 14-\mathrm{Rh} 1$ & $72.01(19)$ & $\mathrm{F} 16-\mathrm{C} 43-\mathrm{C} 40$ & $116.0(4)$ \\
\hline $\mathrm{C} 10-\mathrm{C} 14-\mathrm{Rh} 1$ & $74.89(19)$ & $\mathrm{F} 17 \mathrm{~A}-\mathrm{C} 43-\mathrm{C} 40$ & $114.9(5)$ \\
\hline $\mathrm{C} 19-\mathrm{C} 14-\mathrm{Rh} 1$ & $125.0(2)$ & $\mathrm{F} 18 \mathrm{~A}-\mathrm{C} 43-\mathrm{C} 40$ & $114.1(4)$ \\
\hline $\mathrm{C} 10-\mathrm{C} 15-\mathrm{H} 15 \mathrm{~A}$ & 109.5 & $\mathrm{~F} 17-\mathrm{C} 43-\mathrm{C} 40$ & $111.2(4)$ \\
\hline $\mathrm{C} 10-\mathrm{C} 15-\mathrm{H} 15 \mathrm{~B}$ & 109.5 & $\mathrm{~F} 18-\mathrm{C} 43-\mathrm{C} 40$ & $110.0(4)$ \\
\hline $\mathrm{H} 15 \mathrm{~A}-\mathrm{C} 15-\mathrm{H} 15 \mathrm{~B}$ & 109.5 & $\mathrm{~F} 16 \mathrm{~A}-\mathrm{C} 43-\mathrm{C} 40$ & $108.3(4)$ \\
\hline $\mathrm{C} 10-\mathrm{C} 15-\mathrm{H} 15 \mathrm{C}$ & 109.5 & $\mathrm{C} 49-\mathrm{C} 44-\mathrm{C} 45$ & $116.1(3)$ \\
\hline $\mathrm{H} 15 \mathrm{~A}-\mathrm{C} 15-\mathrm{H} 15 \mathrm{C}$ & 109.5 & $\mathrm{C} 49-\mathrm{C} 44-\mathrm{B} 1$ & $119.7(3)$ \\
\hline $\mathrm{H} 15 \mathrm{~B}-\mathrm{C} 15-\mathrm{H} 15 \mathrm{C}$ & 109.5 & $\mathrm{C} 45-\mathrm{C} 44-\mathrm{B} 1$ & $123.8(3)$ \\
\hline $\mathrm{C} 11-\mathrm{C} 16-\mathrm{H} 16 \mathrm{~A}$ & 109.5 & $\mathrm{C} 46-\mathrm{C} 45-\mathrm{C} 44$ & $121.6(3)$ \\
\hline $\mathrm{C} 11-\mathrm{C} 16-\mathrm{H} 16 \mathrm{~B}$ & 109.5 & $\mathrm{C} 46-\mathrm{C} 45-\mathrm{H} 45$ & 119.2 \\
\hline $\mathrm{H} 16 \mathrm{~A}-\mathrm{C} 16-\mathrm{H} 16 \mathrm{~B}$ & 109.5 & $\mathrm{C} 44-\mathrm{C} 45-\mathrm{H} 45$ & 119.2 \\
\hline $\mathrm{C} 11-\mathrm{C} 16-\mathrm{H} 16 \mathrm{C}$ & 109.5 & $\mathrm{C} 47-\mathrm{C} 46-\mathrm{C} 45$ & $121.2(3)$ \\
\hline $\mathrm{H} 16 \mathrm{~A}-\mathrm{C} 16-\mathrm{H} 16 \mathrm{C}$ & 109.5 & $\mathrm{C} 47-\mathrm{C} 46-\mathrm{C} 50$ & $118.4(3)$ \\
\hline $\mathrm{H} 16 \mathrm{~B}-\mathrm{C} 16-\mathrm{H} 16 \mathrm{C}$ & 109.5 & $\mathrm{C} 45-\mathrm{C} 46-\mathrm{C} 50$ & $120.4(3)$ \\
\hline $\mathrm{C} 12-\mathrm{C} 17-\mathrm{H} 17 \mathrm{~A}$ & 109.5 & $\mathrm{C} 46-\mathrm{C} 47-\mathrm{C} 48$ & $118.0(3)$ \\
\hline $\mathrm{C} 12-\mathrm{C} 17-\mathrm{H} 17 \mathrm{~B}$ & 109.5 & $\mathrm{C} 46-\mathrm{C} 47-\mathrm{H} 47$ & 121.0 \\
\hline $\mathrm{H} 17 \mathrm{~A}-\mathrm{C} 17-\mathrm{H} 17 \mathrm{~B}$ & 109.5 & $\mathrm{C} 48-\mathrm{C} 47-\mathrm{H} 47$ & 121.0 \\
\hline $\mathrm{C} 12-\mathrm{C} 17-\mathrm{H} 17 \mathrm{C}$ & 109.5 & $\mathrm{C} 47-\mathrm{C} 48-\mathrm{C} 49$ & $120.8(3)$ \\
\hline $\mathrm{H} 17 \mathrm{~A}-\mathrm{C} 17-\mathrm{H} 17 \mathrm{C}$ & 109.5 & $\mathrm{C} 47-\mathrm{C} 48-\mathrm{C} 51$ & $119.6(3)$ \\
\hline $\mathrm{H} 17 \mathrm{~B}-\mathrm{C} 17-\mathrm{H} 17 \mathrm{C}$ & 109.5 & $\mathrm{C} 49-\mathrm{C} 48-\mathrm{C} 51$ & $119.6(3)$ \\
\hline $\mathrm{C} 13-\mathrm{C} 18-\mathrm{H} 18 \mathrm{~A}$ & 109.5 & $\mathrm{C} 48-\mathrm{C} 49-\mathrm{C} 44$ & $122.3(3)$ \\
\hline $\mathrm{C} 13-\mathrm{C} 18-\mathrm{H} 18 \mathrm{~B}$ & 109.5 & $\mathrm{C} 48-\mathrm{C} 49-\mathrm{H} 49$ & 118.9 \\
\hline $\mathrm{H} 18 \mathrm{~A}-\mathrm{C} 18-\mathrm{H} 18 \mathrm{~B}$ & 109.5 & $\mathrm{C} 44-\mathrm{C} 49-\mathrm{H} 49$ & 118.9 \\
\hline
\end{tabular}




\begin{tabular}{|c|c|c|c|}
\hline $\mathrm{C} 13-\mathrm{C} 18-\mathrm{H} 18 \mathrm{C}$ & 109.5 & $\mathrm{~F} 20-\mathrm{C} 50-\mathrm{F} 19$ & $106.8(3)$ \\
\hline $\mathrm{H} 18 \mathrm{~A}-\mathrm{C} 18-\mathrm{H} 18 \mathrm{C}$ & 109.5 & $\mathrm{~F} 20-\mathrm{C} 50-\mathrm{F} 21$ & $105.9(3)$ \\
\hline $\mathrm{H} 18 \mathrm{~B}-\mathrm{C} 18-\mathrm{H} 18 \mathrm{C}$ & 109.5 & $\mathrm{~F} 19-\mathrm{C} 50-\mathrm{F} 21$ & $105.7(3)$ \\
\hline $\mathrm{C} 14-\mathrm{C} 19-\mathrm{H} 19 \mathrm{~A}$ & 109.5 & $\mathrm{~F} 20-\mathrm{C} 50-\mathrm{C} 46$ & $112.4(3)$ \\
\hline $\mathrm{C} 14-\mathrm{C} 19-\mathrm{H} 19 \mathrm{~B}$ & 109.5 & $\mathrm{~F} 19-\mathrm{C} 50-\mathrm{C} 46$ & $112.4(3)$ \\
\hline H19A-C19-H19B & 109.5 & $\mathrm{~F} 21-\mathrm{C} 50-\mathrm{C} 46$ & $113.1(3)$ \\
\hline $\mathrm{C} 14-\mathrm{C} 19-\mathrm{H} 19 \mathrm{C}$ & 109.5 & $\mathrm{~F} 22 \mathrm{~A}-\mathrm{C} 51-\mathrm{F} 23 \mathrm{~A}$ & $118.1(5)$ \\
\hline $\mathrm{H} 19 \mathrm{~A}-\mathrm{C} 19-\mathrm{H} 19 \mathrm{C}$ & 109.5 & $\mathrm{~F} 24-\mathrm{C} 51-\mathrm{F} 22$ & $115.1(6)$ \\
\hline $\mathrm{H} 19 \mathrm{~B}-\mathrm{C} 19-\mathrm{H} 19 \mathrm{C}$ & 109.5 & $\mathrm{~F} 24-\mathrm{C} 51-\mathrm{F} 23$ & $103.0(5)$ \\
\hline $\mathrm{C} 21-\mathrm{C} 20-\mathrm{C} 25$ & $116.2(3)$ & $\mathrm{F} 22-\mathrm{C} 51-\mathrm{F} 23$ & $99.3(5)$ \\
\hline $\mathrm{C} 21-\mathrm{C} 20-\mathrm{B} 1$ & $122.7(3)$ & $\mathrm{F} 22 \mathrm{~A}-\mathrm{C} 51-\mathrm{F} 24 \mathrm{~A}$ & $100.1(6)$ \\
\hline $\mathrm{C} 25-\mathrm{C} 20-\mathrm{B} 1$ & $120.7(3)$ & $\mathrm{F} 23 \mathrm{~A}-\mathrm{C} 51-\mathrm{F} 24 \mathrm{~A}$ & $93.0(5)$ \\
\hline $\mathrm{C} 22-\mathrm{C} 21-\mathrm{C} 20$ & $121.7(3)$ & $\mathrm{F} 24-\mathrm{C} 51-\mathrm{C} 48$ & $118.1(4)$ \\
\hline $\mathrm{C} 22-\mathrm{C} 21-\mathrm{H} 21$ & 119.1 & $\mathrm{~F} 22 \mathrm{~A}-\mathrm{C} 51-\mathrm{C} 48$ & $118.9(5)$ \\
\hline $\mathrm{C} 20-\mathrm{C} 21-\mathrm{H} 21$ & 119.1 & $\mathrm{~F} 23 \mathrm{~A}-\mathrm{C} 51-\mathrm{C} 48$ & $114.0(4)$ \\
\hline $\mathrm{C} 23-\mathrm{C} 22-\mathrm{C} 21$ & $121.3(3)$ & $\mathrm{F} 22-\mathrm{C} 51-\mathrm{C} 48$ & $110.5(4)$ \\
\hline $\mathrm{C} 23-\mathrm{C} 22-\mathrm{C} 26$ & $119.6(3)$ & F23-C51-C48 & $108.6(4)$ \\
\hline $\mathrm{C} 21-\mathrm{C} 22-\mathrm{C} 26$ & $119.1(3)$ & $\mathrm{F} 24 \mathrm{~A}-\mathrm{C} 51-\mathrm{C} 48$ & $106.9(4)$ \\
\hline $\mathrm{C} 22-\mathrm{C} 23-\mathrm{C} 24$ & $118.1(3)$ & $\mathrm{N} 2-\mathrm{C} 52-\mathrm{C} 53$ & $179.8(5)$ \\
\hline $\mathrm{C} 22-\mathrm{C} 23-\mathrm{H} 23$ & 121.0 & $\mathrm{C} 52-\mathrm{C} 53-\mathrm{H} 53 \mathrm{~A}$ & 109.5 \\
\hline $\mathrm{C} 24-\mathrm{C} 23-\mathrm{H} 23$ & 121.0 & $\mathrm{C} 52-\mathrm{C} 53-\mathrm{H} 53 \mathrm{~B}$ & 109.5 \\
\hline $\mathrm{C} 25-\mathrm{C} 24-\mathrm{C} 23$ & $120.5(3)$ & $\mathrm{H} 53 \mathrm{~A}-\mathrm{C} 53-\mathrm{H} 53 \mathrm{~B}$ & 109.5 \\
\hline $\mathrm{C} 25-\mathrm{C} 24-\mathrm{C} 27$ & $121.1(3)$ & $\mathrm{C} 52-\mathrm{C} 53-\mathrm{H} 53 \mathrm{C}$ & 109.5 \\
\hline $\mathrm{C} 23-\mathrm{C} 24-\mathrm{C} 27$ & $118.4(3)$ & $\mathrm{H} 53 \mathrm{~A}-\mathrm{C} 53-\mathrm{H} 53 \mathrm{C}$ & 109.5 \\
\hline $\mathrm{C} 24-\mathrm{C} 25-\mathrm{C} 20$ & $122.3(3)$ & $\mathrm{H} 53 \mathrm{~B}-\mathrm{C} 53-\mathrm{H} 53 \mathrm{C}$ & 109.5 \\
\hline $\mathrm{C} 24-\mathrm{C} 25-\mathrm{H} 25$ & 118.9 & $\mathrm{C} 36-\mathrm{B} 1-\mathrm{C} 44$ & $113.4(3)$ \\
\hline $\mathrm{C} 20-\mathrm{C} 25-\mathrm{H} 25$ & 118.9 & $\mathrm{C} 36-\mathrm{B} 1-\mathrm{C} 28$ & $113.3(3)$ \\
\hline $\mathrm{F} 1-\mathrm{C} 26-\mathrm{F} 3$ & $106.7(3)$ & $\mathrm{C} 44-\mathrm{B} 1-\mathrm{C} 28$ & $104.7(2)$ \\
\hline $\mathrm{F} 1-\mathrm{C} 26-\mathrm{F} 2$ & $105.8(3)$ & $\mathrm{C} 36-\mathrm{B} 1-\mathrm{C} 20$ & $102.3(2)$ \\
\hline $\mathrm{F} 3-\mathrm{C} 26-\mathrm{F} 2$ & $106.6(3)$ & $\mathrm{C} 44-\mathrm{B} 1-\mathrm{C} 20$ & $110.1(3)$ \\
\hline $\mathrm{F} 1-\mathrm{C} 26-\mathrm{C} 22$ & $113.2(3)$ & $\mathrm{C} 28-\mathrm{B} 1-\mathrm{C} 20$ & $113.2(3)$ \\
\hline $\mathrm{F} 3-\mathrm{C} 26-\mathrm{C} 22$ & $113.1(3)$ & $\mathrm{C} 3-\mathrm{N} 1-\mathrm{C} 1$ & $121.2(3)$ \\
\hline $\mathrm{F} 2-\mathrm{C} 26-\mathrm{C} 22$ & $111.0(3)$ & $\mathrm{C} 3-\mathrm{N} 1-\mathrm{Rh} 1$ & $117.1(2)$ \\
\hline $\mathrm{F} 6 \mathrm{~A}-\mathrm{C} 27-\mathrm{F} 5 \mathrm{~A}$ & $110.9(5)$ & $\mathrm{C} 1-\mathrm{N} 1-\mathrm{Rh} 1$ & $121.7(2)$ \\
\hline $\mathrm{F} 4-\mathrm{C} 27-\mathrm{F} 5$ & $106.8(5)$ & $\mathrm{C} 52-\mathrm{N} 2-\mathrm{Rh} 1$ & $173.3(3)$ \\
\hline $\mathrm{F} 6 \mathrm{~A}-\mathrm{C} 27-\mathrm{F} 4 \mathrm{~A}$ & $102.6(5)$ & C9-Rh1-N2 & $89.86(13)$ \\
\hline $\mathrm{F} 5 \mathrm{~A}-\mathrm{C} 27-\mathrm{F} 4 \mathrm{~A}$ & $103.5(5)$ & C9-Rh1-N1 & $78.26(13)$ \\
\hline
\end{tabular}




\begin{tabular}{|c|c|c|c|}
\hline $\mathrm{F} 4-\mathrm{C} 27-\mathrm{F} 6$ & $104.1(4)$ & $\mathrm{N} 2-\mathrm{Rh} 1-\mathrm{N} 1$ & $87.00(12)$ \\
\hline $\mathrm{F} 5-\mathrm{C} 27-\mathrm{F} 6$ & $106.2(5)$ & C9-Rh1-C14 & $112.65(13)$ \\
\hline $\mathrm{F} 6 \mathrm{~A}-\mathrm{C} 27-\mathrm{C} 24$ & $115.5(4)$ & $\mathrm{N} 2-\mathrm{Rh} 1-\mathrm{C} 14$ & $157.38(13)$ \\
\hline $\mathrm{F} 4-\mathrm{C} 27-\mathrm{C} 24$ & $114.6(4)$ & $\mathrm{N} 1-\mathrm{Rh} 1-\mathrm{C} 14$ & 99.35 (12) \\
\hline $\mathrm{F} 5 \mathrm{~A}-\mathrm{C} 27-\mathrm{C} 24$ & $114.2(5)$ & C9-Rh1-C12 & $109.24(13)$ \\
\hline $\mathrm{F} 5-\mathrm{C} 27-\mathrm{C} 24$ & $114.9(5)$ & $\mathrm{N} 2-\mathrm{Rh} 1-\mathrm{C} 12$ & $106.90(13)$ \\
\hline $\mathrm{F} 4 \mathrm{~A}-\mathrm{C} 27-\mathrm{C} 24$ & $108.7(4)$ & $\mathrm{N} 1-\mathrm{Rh} 1-\mathrm{C} 12$ & $163.86(12)$ \\
\hline $\mathrm{F} 6-\mathrm{C} 27-\mathrm{C} 24$ & $109.4(4)$ & $\mathrm{C} 14-\mathrm{Rh} 1-\mathrm{C} 12$ & $64.69(13)$ \\
\hline $\mathrm{C} 33-\mathrm{C} 28-\mathrm{C} 29$ & $115.9(3)$ & C9-Rh1-C13 & $92.91(13)$ \\
\hline $\mathrm{C} 33-\mathrm{C} 28-\mathrm{B} 1$ & $121.2(3)$ & $\mathrm{N} 2-\mathrm{Rh} 1-\mathrm{C} 13$ & $143.26(13)$ \\
\hline $\mathrm{C} 29-\mathrm{C} 28-\mathrm{B} 1$ & $122.6(3)$ & $\mathrm{N} 1-\mathrm{Rh} 1-\mathrm{C} 13$ & $129.40(12)$ \\
\hline $\mathrm{C} 30-\mathrm{C} 29-\mathrm{C} 28$ & $121.9(3)$ & $\mathrm{C} 14-\mathrm{Rh} 1-\mathrm{C} 13$ & $38.69(13)$ \\
\hline $\mathrm{C} 30-\mathrm{C} 29-\mathrm{H} 29$ & 119.0 & $\mathrm{C} 12-\mathrm{Rh} 1-\mathrm{C} 13$ & $38.31(13)$ \\
\hline $\mathrm{C} 28-\mathrm{C} 29-\mathrm{H} 29$ & 119.0 & C9-Rh1-C11 & $147.40(14)$ \\
\hline $\mathrm{C} 31-\mathrm{C} 30-\mathrm{C} 29$ & $120.9(3)$ & $\mathrm{N} 2-\mathrm{Rh} 1-\mathrm{C} 11$ & $96.81(12)$ \\
\hline $\mathrm{C} 31-\mathrm{C} 30-\mathrm{C} 34$ & $118.1(3)$ & $\mathrm{N} 1-\mathrm{Rh} 1-\mathrm{C} 11$ & 133.77 (12) \\
\hline $\mathrm{C} 29-\mathrm{C} 30-\mathrm{C} 34$ & $121.0(3)$ & $\mathrm{C} 14-\mathrm{Rh} 1-\mathrm{C} 11$ & $63.17(13)$ \\
\hline $\mathrm{C} 30-\mathrm{C} 31-\mathrm{C} 32$ & $118.4(3)$ & $\mathrm{C} 12-\mathrm{Rh} 1-\mathrm{C} 11$ & $38.37(13)$ \\
\hline $\mathrm{C} 30-\mathrm{C} 31-\mathrm{H} 31$ & 120.8 & $\mathrm{C} 13-\mathrm{Rh} 1-\mathrm{C} 11$ & $63.28(13)$ \\
\hline $\mathrm{C} 32-\mathrm{C} 31-\mathrm{H} 31$ & 120.8 & $\mathrm{C} 9-\mathrm{Rh} 1-\mathrm{C} 10$ & $151.25(13)$ \\
\hline $\mathrm{C} 31-\mathrm{C} 32-\mathrm{C} 33$ & $120.4(3)$ & $\mathrm{N} 2-\mathrm{Rh} 1-\mathrm{C} 10$ & $118.86(12)$ \\
\hline $\mathrm{C} 31-\mathrm{C} 32-\mathrm{C} 35$ & $119.4(3)$ & $\mathrm{N} 1-\mathrm{Rh} 1-\mathrm{C} 10$ & $103.00(12)$ \\
\hline $\mathrm{C} 33-\mathrm{C} 32-\mathrm{C} 35$ & $120.2(3)$ & $\mathrm{C} 14-\mathrm{Rh} 1-\mathrm{C} 10$ & $38.60(13)$ \\
\hline $\mathrm{C} 32-\mathrm{C} 33-\mathrm{C} 28$ & $122.3(3)$ & $\mathrm{C} 12-\mathrm{Rh} 1-\mathrm{C} 10$ & $63.38(13)$ \\
\hline $\mathrm{C} 32-\mathrm{C} 33-\mathrm{H} 33$ & 118.8 & $\mathrm{C} 13-\mathrm{Rh} 1-\mathrm{C} 10$ & $63.73(13)$ \\
\hline $\mathrm{C} 28-\mathrm{C} 33-\mathrm{H} 33$ & 118.8 & $\mathrm{C} 11-\mathrm{Rh} 1-\mathrm{C} 10$ & $36.27(13)$ \\
\hline $\mathrm{N} 1-\mathrm{C} 3-\mathrm{C} 4-\mathrm{C} 5$ & $-176.8(3)$ & $\begin{array}{l}\mathrm{C} 29-\mathrm{C} 30-\mathrm{C} 34- \\
\text { F9A }\end{array}$ & $128.9(4)$ \\
\hline $\mathrm{C} 2-\mathrm{C} 3-\mathrm{C} 4-\mathrm{C} 5$ & $0.8(6)$ & $\begin{array}{l}\mathrm{C} 31-\mathrm{C} 32-\mathrm{C} 35- \\
\mathrm{F} 12\end{array}$ & $93.4(5)$ \\
\hline $\mathrm{N} 1-\mathrm{C} 3-\mathrm{C} 4-\mathrm{C} 9$ & $-1.1(5)$ & $\begin{array}{l}\mathrm{C} 33-\mathrm{C} 32-\mathrm{C} 35- \\
\mathrm{F} 12\end{array}$ & $-84.8(5)$ \\
\hline $\mathrm{C} 2-\mathrm{C} 3-\mathrm{C} 4-\mathrm{C} 9$ & $176.6(3)$ & $\begin{array}{l}\mathrm{C} 31-\mathrm{C} 32-\mathrm{C} 35- \\
\mathrm{F} 10 \mathrm{~A}\end{array}$ & $-127.2(4)$ \\
\hline $\mathrm{C} 9-\mathrm{C} 4-\mathrm{C} 5-\mathrm{C} 6$ & $-0.9(5)$ & $\begin{array}{l}\mathrm{C} 33-\mathrm{C} 32-\mathrm{C} 35- \\
\mathrm{F} 10 \mathrm{~A}\end{array}$ & $54.6(5)$ \\
\hline $\mathrm{C} 3-\mathrm{C} 4-\mathrm{C} 5-\mathrm{C} 6$ & $174.6(4)$ & $\begin{array}{l}\mathrm{C} 31-\mathrm{C} 32-\mathrm{C} 35- \\
\text { F11A }\end{array}$ & $-2.1(5)$ \\
\hline
\end{tabular}




\begin{tabular}{|c|c|c|c|}
\hline $\mathrm{C} 4-\mathrm{C} 5-\mathrm{C} 6-\mathrm{C} 7$ & $-0.7(6)$ & $\begin{array}{l}\mathrm{C} 33-\mathrm{C} 32-\mathrm{C} 35- \\
\mathrm{F} 11 \mathrm{~A}\end{array}$ & $179.7(4)$ \\
\hline $\mathrm{C} 5-\mathrm{C} 6-\mathrm{C} 7-\mathrm{C} 8$ & $1.1(6)$ & $\begin{array}{l}\mathrm{C} 31-\mathrm{C} 32-\mathrm{C} 35- \\
\mathrm{F} 10\end{array}$ & $-144.9(4)$ \\
\hline $\mathrm{C} 6-\mathrm{C} 7-\mathrm{C} 8-\mathrm{C} 9$ & $0.1(5)$ & $\begin{array}{l}\text { C33-C32-C35- } \\
\text { F10 }\end{array}$ & $36.9(5)$ \\
\hline $\mathrm{C} 7-\mathrm{C} 8-\mathrm{C} 9-\mathrm{C} 4$ & $-1.7(5)$ & $\begin{array}{l}\text { C31-C32-C35- } \\
\text { F11 }\end{array}$ & $-30.3(5)$ \\
\hline $\mathrm{C} 7-\mathrm{C} 8-\mathrm{C} 9-\mathrm{Rh} 1$ & $177.6(3)$ & $\begin{array}{l}\mathrm{C} 33-\mathrm{C} 32-\mathrm{C} 35- \\
\mathrm{F} 11\end{array}$ & $151.6(4)$ \\
\hline $\mathrm{C} 5-\mathrm{C} 4-\mathrm{C} 9-\mathrm{C} 8$ & $2.1(5)$ & $\begin{array}{l}\text { C31-C32-C35- } \\
\text { F12A }\end{array}$ & $115.3(4)$ \\
\hline $\mathrm{C} 3-\mathrm{C} 4-\mathrm{C} 9-\mathrm{C} 8$ & $-173.8(3)$ & $\begin{array}{l}\text { C33-C32-C35- } \\
\text { F12A }\end{array}$ & $-62.9(5)$ \\
\hline $\mathrm{C} 5-\mathrm{C} 4-\mathrm{C} 9-\mathrm{Rh} 1$ & $-177.3(3)$ & $\begin{array}{l}\mathrm{C} 41-\mathrm{C} 36-\mathrm{C} 37- \\
\mathrm{C} 38\end{array}$ & $1.1(4)$ \\
\hline $\mathrm{C} 3-\mathrm{C} 4-\mathrm{C} 9-\mathrm{Rh} 1$ & $6.8(4)$ & $\mathrm{B} 1-\mathrm{C} 36-\mathrm{C} 37-\mathrm{C} 38$ & $172.3(3)$ \\
\hline $\begin{array}{l}\mathrm{C} 14-\mathrm{C} 10-\mathrm{C} 11- \\
\mathrm{C} 12\end{array}$ & $-1.2(4)$ & $\begin{array}{l}\mathrm{C} 36-\mathrm{C} 37-\mathrm{C} 38- \\
\mathrm{C} 39\end{array}$ & $1.0(5)$ \\
\hline $\begin{array}{l}\mathrm{C} 15-\mathrm{C} 10-\mathrm{C} 11- \\
\mathrm{C} 12\end{array}$ & $176.2(3)$ & $\begin{array}{l}\mathrm{C} 36-\mathrm{C} 37-\mathrm{C} 38- \\
\mathrm{C} 42\end{array}$ & $-176.1(3)$ \\
\hline $\begin{array}{l}\mathrm{Rh} 1-\mathrm{C} 10-\mathrm{C} 11- \\
\mathrm{C} 12\end{array}$ & $-58.3(2)$ & $\begin{array}{l}\mathrm{C} 37-\mathrm{C} 38-\mathrm{C} 39- \\
\mathrm{C} 40\end{array}$ & $-1.6(5)$ \\
\hline $\begin{array}{l}\mathrm{C} 14-\mathrm{C} 10-\mathrm{C} 11- \\
\mathrm{C} 16\end{array}$ & $179.6(3)$ & $\begin{array}{l}\mathrm{C} 42-\mathrm{C} 38-\mathrm{C} 39- \\
\mathrm{C} 40\end{array}$ & $175.6(3)$ \\
\hline $\begin{array}{l}\mathrm{C} 15-\mathrm{C} 10-\mathrm{C} 11- \\
\mathrm{C} 16\end{array}$ & $-3.0(6)$ & $\begin{array}{l}\mathrm{C} 38-\mathrm{C} 39-\mathrm{C} 40- \\
\mathrm{C} 41\end{array}$ & $0.0(5)$ \\
\hline $\begin{array}{l}\mathrm{Rh} 1-\mathrm{C} 10-\mathrm{C} 11- \\
\mathrm{C} 16\end{array}$ & $122.5(4)$ & $\begin{array}{l}\mathrm{C} 38-\mathrm{C} 39-\mathrm{C} 40- \\
\mathrm{C} 43\end{array}$ & $-177.8(3)$ \\
\hline $\begin{array}{l}\mathrm{C} 14-\mathrm{C} 10-\mathrm{C} 11- \\
\mathrm{Rh} 1\end{array}$ & $57.1(2)$ & $\begin{array}{l}\mathrm{C} 39-\mathrm{C} 40-\mathrm{C} 41- \\
\mathrm{C} 36\end{array}$ & $2.3(5)$ \\
\hline $\begin{array}{l}\mathrm{C} 15-\mathrm{C} 10-\mathrm{C} 11- \\
\mathrm{Rh} 1\end{array}$ & $-125.5(4)$ & $\begin{array}{l}\mathrm{C} 43-\mathrm{C} 40-\mathrm{C} 41- \\
\mathrm{C} 36\end{array}$ & $-180.0(3)$ \\
\hline $\begin{array}{l}\mathrm{C} 10-\mathrm{C} 11-\mathrm{C} 12- \\
\mathrm{C} 13\end{array}$ & $-2.6(4)$ & $\begin{array}{l}\mathrm{C} 37-\mathrm{C} 36-\mathrm{C} 41- \\
\mathrm{C} 40\end{array}$ & $-2.7(5)$ \\
\hline $\begin{array}{l}\mathrm{C} 16-\mathrm{C} 11-\mathrm{C} 12- \\
\mathrm{C} 13\end{array}$ & $176.6(3)$ & $\mathrm{B} 1-\mathrm{C} 36-\mathrm{C} 41-\mathrm{C} 40$ & $-173.7(3)$ \\
\hline $\begin{array}{l}\mathrm{Rh} 1-\mathrm{C} 11-\mathrm{C} 12- \\
\mathrm{C} 13\end{array}$ & $-63.5(2)$ & $\begin{array}{l}\text { C39-C38-C42- } \\
\text { F14A }\end{array}$ & $37(2)$ \\
\hline $\begin{array}{l}\mathrm{C} 10-\mathrm{C} 11-\mathrm{C} 12- \\
\mathrm{C} 17\end{array}$ & $-173.0(3)$ & $\begin{array}{l}\mathrm{C} 37-\mathrm{C} 38-\mathrm{C} 42- \\
\mathrm{F} 14 \mathrm{~A}\end{array}$ & $-146(2)$ \\
\hline $\begin{array}{l}\mathrm{C} 16-\mathrm{C} 11-\mathrm{C} 12- \\
\mathrm{C} 17\end{array}$ & $6.3(6)$ & $\begin{array}{l}\mathrm{C} 39-\mathrm{C} 38-\mathrm{C} 42- \\
\text { F15 }\end{array}$ & $-70.2(11)$ \\
\hline $\mathrm{Rh} 1-\mathrm{C} 11-\mathrm{C} 12-$ & $126.2(3)$ & $\mathrm{C} 37-\mathrm{C} 38-\mathrm{C} 42-$ & $107.0(11)$ \\
\hline
\end{tabular}




\begin{tabular}{|c|c|c|c|}
\hline $\mathrm{C} 17$ & & F15 & \\
\hline $\begin{array}{l}\mathrm{C} 10-\mathrm{C} 11-\mathrm{C} 12- \\
\mathrm{Rh} 1\end{array}$ & $60.8(2)$ & $\begin{array}{l}\mathrm{C} 39-\mathrm{C} 38-\mathrm{C} 42- \\
\mathrm{F} 13 \mathrm{~A}\end{array}$ & $167(3)$ \\
\hline $\begin{array}{l}\mathrm{C} 16-\mathrm{C} 11-\mathrm{C} 12- \\
\mathrm{Rh} 1\end{array}$ & $-119.9(3)$ & $\begin{array}{l}\mathrm{C} 37-\mathrm{C} 38-\mathrm{C} 42- \\
\mathrm{F} 13 \mathrm{~A}\end{array}$ & $-15(3)$ \\
\hline $\begin{array}{l}\mathrm{C} 11-\mathrm{C} 12-\mathrm{C} 13- \\
\mathrm{C} 14\end{array}$ & $5.4(4)$ & $\begin{array}{l}\mathrm{C} 39-\mathrm{C} 38-\mathrm{C} 42- \\
\mathrm{F} 13\end{array}$ & $158(3)$ \\
\hline $\begin{array}{l}\mathrm{C} 17-\mathrm{C} 12-\mathrm{C} 13- \\
\mathrm{C} 14\end{array}$ & $175.5(3)$ & $\begin{array}{l}\mathrm{C} 37-\mathrm{C} 38-\mathrm{C} 42- \\
\text { F13 }\end{array}$ & $-24(3)$ \\
\hline $\begin{array}{l}\mathrm{Rh} 1-\mathrm{C} 12-\mathrm{C} 13- \\
\mathrm{C} 14\end{array}$ & $-59.6(2)$ & $\begin{array}{l}\mathrm{C} 39-\mathrm{C} 38-\mathrm{C} 42- \\
\mathrm{F} 14\end{array}$ & $43.9(15)$ \\
\hline $\begin{array}{l}\mathrm{C} 11-\mathrm{C} 12-\mathrm{C} 13- \\
\mathrm{C} 18\end{array}$ & $-172.4(3)$ & $\begin{array}{l}\mathrm{C} 37-\mathrm{C} 38-\mathrm{C} 42- \\
\mathrm{F} 14\end{array}$ & $-138.9(15)$ \\
\hline $\begin{array}{l}\mathrm{C} 17-\mathrm{C} 12-\mathrm{C} 13- \\
\mathrm{C} 18\end{array}$ & $-2.4(6)$ & $\begin{array}{l}\mathrm{C} 39-\mathrm{C} 38-\mathrm{C} 42- \\
\mathrm{F} 15 \mathrm{~A}\end{array}$ & $-88.2(12)$ \\
\hline $\begin{array}{l}\mathrm{Rh} 1-\mathrm{C} 12-\mathrm{C} 13- \\
\mathrm{C} 18\end{array}$ & $122.5(3)$ & $\begin{array}{l}\mathrm{C} 37-\mathrm{C} 38-\mathrm{C} 42- \\
\mathrm{F} 15 \mathrm{~A}\end{array}$ & $89.0(12)$ \\
\hline $\begin{array}{l}\mathrm{C} 11-\mathrm{C} 12-\mathrm{C} 13- \\
\mathrm{Rh} 1\end{array}$ & $65.1(2)$ & $\begin{array}{l}\mathrm{C} 39-\mathrm{C} 40-\mathrm{C} 43- \\
\mathrm{F} 16\end{array}$ & $-31.4(6)$ \\
\hline $\begin{array}{l}\mathrm{C} 17-\mathrm{C} 12-\mathrm{C} 13- \\
\mathrm{Rh} 1\end{array}$ & $-124.9(4)$ & $\begin{array}{l}\mathrm{C} 41-\mathrm{C} 40-\mathrm{C} 43- \\
\mathrm{F} 16\end{array}$ & $150.8(4)$ \\
\hline $\begin{array}{l}\mathrm{C} 12-\mathrm{C} 13-\mathrm{C} 14- \\
\mathrm{C} 10\end{array}$ & $-6.2(4)$ & $\begin{array}{l}\mathrm{C} 39-\mathrm{C} 40-\mathrm{C} 43- \\
\mathrm{F} 17 \mathrm{~A}\end{array}$ & $-169.5(5)$ \\
\hline $\begin{array}{l}\mathrm{C} 18-\mathrm{C} 13-\mathrm{C} 14- \\
\mathrm{C} 10\end{array}$ & $171.7(3)$ & $\begin{array}{l}\mathrm{C} 41-\mathrm{C} 40-\mathrm{C} 43- \\
\text { F17A }\end{array}$ & $12.7(6)$ \\
\hline $\begin{array}{l}\mathrm{Rh} 1-\mathrm{C} 13-\mathrm{C} 14- \\
\mathrm{C} 10\end{array}$ & $-66.7(2)$ & $\begin{array}{l}\mathrm{C} 39-\mathrm{C} 40-\mathrm{C} 43- \\
\text { F18A }\end{array}$ & $66.3(5)$ \\
\hline $\begin{array}{l}\mathrm{C} 12-\mathrm{C} 13-\mathrm{C} 14- \\
\mathrm{C} 19\end{array}$ & $-178.9(3)$ & $\begin{array}{l}\mathrm{C} 41-\mathrm{C} 40-\mathrm{C} 43- \\
\mathrm{F} 18 \mathrm{~A}\end{array}$ & $-111.4(4)$ \\
\hline $\begin{array}{l}\mathrm{C} 18-\mathrm{C} 13-\mathrm{C} 14- \\
\mathrm{C} 19\end{array}$ & $-1.0(5)$ & $\begin{array}{l}\mathrm{C} 39-\mathrm{C} 40-\mathrm{C} 43- \\
\mathrm{F} 17\end{array}$ & $-153.1(5)$ \\
\hline $\begin{array}{l}\mathrm{Rh} 1-\mathrm{C} 13-\mathrm{C} 14- \\
\mathrm{C} 19\end{array}$ & $120.6(3)$ & $\begin{array}{l}\mathrm{C} 41-\mathrm{C} 40-\mathrm{C} 43- \\
\mathrm{F} 17\end{array}$ & $29.1(6)$ \\
\hline $\begin{array}{l}\mathrm{C} 12-\mathrm{C} 13-\mathrm{C} 14- \\
\mathrm{Rh} 1\end{array}$ & $60.5(2)$ & $\begin{array}{l}\mathrm{C} 39-\mathrm{C} 40-\mathrm{C} 43- \\
\mathrm{F} 18\end{array}$ & $88.6(4)$ \\
\hline $\begin{array}{l}\mathrm{C} 18-\mathrm{C} 13-\mathrm{C} 14- \\
\mathrm{Rh} 1\end{array}$ & $-121.6(3)$ & $\begin{array}{l}\mathrm{C} 41-\mathrm{C} 40-\mathrm{C} 43- \\
\mathrm{F} 18\end{array}$ & $-89.2(4)$ \\
\hline $\begin{array}{l}\mathrm{C} 11-\mathrm{C} 10-\mathrm{C} 14- \\
\mathrm{C} 13\end{array}$ & $4.6(4)$ & $\begin{array}{l}\mathrm{C} 39-\mathrm{C} 40-\mathrm{C} 43- \\
\text { F16A }\end{array}$ & $-50.3(5)$ \\
\hline $\begin{array}{l}\mathrm{C} 15-\mathrm{C} 10-\mathrm{C} 14- \\
\mathrm{C} 13\end{array}$ & $-172.9(3)$ & $\begin{array}{l}\mathrm{C} 41-\mathrm{C} 40-\mathrm{C} 43- \\
\mathrm{F} 16 \mathrm{~A}\end{array}$ & $131.9(4)$ \\
\hline $\begin{array}{l}\mathrm{Rh} 1-\mathrm{C} 10-\mathrm{C} 14- \\
\mathrm{C} 13\end{array}$ & $64.8(2)$ & $\begin{array}{l}\mathrm{C} 49-\mathrm{C} 44-\mathrm{C} 45- \\
\mathrm{C} 46\end{array}$ & $0.3(5)$ \\
\hline $\begin{array}{l}\mathrm{C} 11-\mathrm{C} 10-\mathrm{C} 14- \\
\mathrm{C} 19\end{array}$ & $177.3(3)$ & $\mathrm{B} 1-\mathrm{C} 44-\mathrm{C} 45-\mathrm{C} 46$ & $172.9(3)$ \\
\hline
\end{tabular}




\begin{tabular}{|c|c|c|c|}
\hline $\begin{array}{l}\mathrm{C} 15-\mathrm{C} 10-\mathrm{C} 14- \\
\mathrm{C} 19\end{array}$ & $-0.1(5)$ & $\begin{array}{l}\mathrm{C} 44-\mathrm{C} 45-\mathrm{C} 46- \\
\mathrm{C} 47\end{array}$ & $-0.7(5)$ \\
\hline $\begin{array}{l}\text { Rh1-C10-C14- } \\
\text { C19 }\end{array}$ & $-122.5(3)$ & $\begin{array}{l}\mathrm{C} 44-\mathrm{C} 45-\mathrm{C} 46- \\
\mathrm{C} 50\end{array}$ & $177.8(3)$ \\
\hline $\begin{array}{l}\mathrm{C} 11-\mathrm{C} 10-\mathrm{C} 14- \\
\mathrm{Rh} 1\end{array}$ & $-60.2(2)$ & $\begin{array}{l}\mathrm{C} 45-\mathrm{C} 46-\mathrm{C} 47- \\
\mathrm{C} 48\end{array}$ & $0.3(5)$ \\
\hline $\begin{array}{l}\mathrm{C} 15-\mathrm{C} 10-\mathrm{C} 14- \\
\mathrm{Rh} 1\end{array}$ & $122.4(3)$ & $\begin{array}{l}\mathrm{C} 50-\mathrm{C} 46-\mathrm{C} 47- \\
\mathrm{C} 48\end{array}$ & $-178.3(3)$ \\
\hline $\begin{array}{l}\mathrm{C} 25-\mathrm{C} 20-\mathrm{C} 21- \\
\mathrm{C} 22\end{array}$ & $-0.7(5)$ & $\begin{array}{l}\mathrm{C} 46-\mathrm{C} 47-\mathrm{C} 48- \\
\mathrm{C} 49\end{array}$ & $0.4(5)$ \\
\hline $\mathrm{B} 1-\mathrm{C} 20-\mathrm{C} 21-\mathrm{C} 22$ & $171.8(3)$ & $\begin{array}{l}\mathrm{C} 46-\mathrm{C} 47-\mathrm{C} 48- \\
\mathrm{C} 51\end{array}$ & $-178.7(3)$ \\
\hline $\begin{array}{l}\mathrm{C} 20-\mathrm{C} 21-\mathrm{C} 22- \\
\mathrm{C} 23\end{array}$ & $0.3(5)$ & $\begin{array}{l}\mathrm{C} 47-\mathrm{C} 48-\mathrm{C} 49- \\
\mathrm{C} 44\end{array}$ & $-0.8(5)$ \\
\hline $\begin{array}{l}\mathrm{C} 20-\mathrm{C} 21-\mathrm{C} 22- \\
\mathrm{C} 26\end{array}$ & $-176.8(3)$ & $\begin{array}{l}\mathrm{C} 51-\mathrm{C} 48-\mathrm{C} 49- \\
\mathrm{C} 44\end{array}$ & $178.4(3)$ \\
\hline $\begin{array}{l}\mathrm{C} 21-\mathrm{C} 22-\mathrm{C} 23- \\
\mathrm{C} 24\end{array}$ & $0.5(5)$ & $\begin{array}{l}\mathrm{C} 45-\mathrm{C} 44-\mathrm{C} 49- \\
\mathrm{C} 48\end{array}$ & $0.4(5)$ \\
\hline $\begin{array}{l}\mathrm{C} 26-\mathrm{C} 22-\mathrm{C} 23- \\
\mathrm{C} 24\end{array}$ & $177.6(3)$ & $\mathrm{B} 1-\mathrm{C} 44-\mathrm{C} 49-\mathrm{C} 48$ & $-172.6(3)$ \\
\hline $\begin{array}{l}\mathrm{C} 22-\mathrm{C} 23-\mathrm{C} 24- \\
\mathrm{C} 25\end{array}$ & $-0.7(5)$ & $\begin{array}{l}\mathrm{C} 47-\mathrm{C} 46-\mathrm{C} 50- \\
\mathrm{F} 20\end{array}$ & $91.9(4)$ \\
\hline $\begin{array}{l}\mathrm{C} 22-\mathrm{C} 23-\mathrm{C} 24- \\
\mathrm{C} 27\end{array}$ & $178.6(3)$ & $\begin{array}{l}\mathrm{C} 45-\mathrm{C} 46-\mathrm{C} 50- \\
\mathrm{F} 20\end{array}$ & $-86.6(4)$ \\
\hline $\begin{array}{l}\mathrm{C} 23-\mathrm{C} 24-\mathrm{C} 25- \\
\mathrm{C} 20\end{array}$ & $0.2(5)$ & $\begin{array}{l}\mathrm{C} 47-\mathrm{C} 46-\mathrm{C} 50- \\
\text { F19 }\end{array}$ & $-28.6(4)$ \\
\hline $\begin{array}{l}\mathrm{C} 27-\mathrm{C} 24-\mathrm{C} 25- \\
\mathrm{C} 20\end{array}$ & $-179.1(3)$ & $\begin{array}{l}\mathrm{C} 45-\mathrm{C} 46-\mathrm{C} 50- \\
\mathrm{F} 19\end{array}$ & $152.9(3)$ \\
\hline $\begin{array}{l}\mathrm{C} 21-\mathrm{C} 20-\mathrm{C} 25- \\
\mathrm{C} 24\end{array}$ & $0.5(5)$ & $\begin{array}{l}\mathrm{C} 47-\mathrm{C} 46-\mathrm{C} 50- \\
\mathrm{F} 21\end{array}$ & $-148.2(3)$ \\
\hline $\mathrm{B} 1-\mathrm{C} 20-\mathrm{C} 25-\mathrm{C} 24$ & $-172.1(3)$ & $\begin{array}{l}\mathrm{C} 45-\mathrm{C} 46-\mathrm{C} 50- \\
\mathrm{F} 21\end{array}$ & $33.3(4)$ \\
\hline $\mathrm{C} 23-\mathrm{C} 22-\mathrm{C} 26-\mathrm{F} 1$ & $145.4(3)$ & $\begin{array}{l}\mathrm{C} 47-\mathrm{C} 48-\mathrm{C} 51- \\
\mathrm{F} 24\end{array}$ & $161.1(5)$ \\
\hline $\mathrm{C} 21-\mathrm{C} 22-\mathrm{C} 26-\mathrm{F} 1$ & $-37.4(4)$ & $\begin{array}{l}\mathrm{C} 49-\mathrm{C} 48-\mathrm{C} 51- \\
\mathrm{F} 24\end{array}$ & $-18.1(7)$ \\
\hline $\mathrm{C} 23-\mathrm{C} 22-\mathrm{C} 26-\mathrm{F} 3$ & $24.0(5)$ & $\begin{array}{l}\mathrm{C} 47-\mathrm{C} 48-\mathrm{C} 51- \\
\mathrm{F} 22 \mathrm{~A}\end{array}$ & $7.6(8)$ \\
\hline $\mathrm{C} 21-\mathrm{C} 22-\mathrm{C} 26-\mathrm{F} 3$ & $-158.9(3)$ & $\begin{array}{l}\mathrm{C} 49-\mathrm{C} 48-\mathrm{C} 51- \\
\mathrm{F} 22 \mathrm{~A}\end{array}$ & $-171.6(6)$ \\
\hline $\mathrm{C} 23-\mathrm{C} 22-\mathrm{C} 26-\mathrm{F} 2$ & $-95.8(4)$ & $\begin{array}{l}\mathrm{C} 47-\mathrm{C} 48-\mathrm{C} 51- \\
\mathrm{F} 23 \mathrm{~A}\end{array}$ & $-139.0(5)$ \\
\hline $\mathrm{C} 21-\mathrm{C} 22-\mathrm{C} 26-\mathrm{F} 2$ & $81.4(4)$ & $\begin{array}{l}\mathrm{C} 49-\mathrm{C} 48-\mathrm{C} 51- \\
\mathrm{F} 23 \mathrm{~A}\end{array}$ & $41.8(6)$ \\
\hline
\end{tabular}




\begin{tabular}{|c|c|c|c|}
\hline $\begin{array}{l}\mathrm{C} 25-\mathrm{C} 24-\mathrm{C} 27- \\
\mathrm{F} 6 \mathrm{~A}\end{array}$ & $-141.8(5)$ & $\begin{array}{l}\text { C47-C48-C51- } \\
\text { F22 }\end{array}$ & $25.7(7)$ \\
\hline $\begin{array}{l}\mathrm{C} 23-\mathrm{C} 24-\mathrm{C} 27- \\
\mathrm{F} 6 \mathrm{~A}\end{array}$ & $38.9(6)$ & $\begin{array}{l}\mathrm{C} 49-\mathrm{C} 48-\mathrm{C} 51- \\
\mathrm{F} 22\end{array}$ & $-153.5(5)$ \\
\hline $\mathrm{C} 25-\mathrm{C} 24-\mathrm{C} 27-\mathrm{F} 4$ & $131.8(5)$ & $\begin{array}{l}\mathrm{C} 47-\mathrm{C} 48-\mathrm{C} 51- \\
\text { F23 }\end{array}$ & $-82.2(5)$ \\
\hline $\mathrm{C} 23-\mathrm{C} 24-\mathrm{C} 27-\mathrm{F} 4$ & $-47.5(6)$ & $\begin{array}{l}\mathrm{C} 49-\mathrm{C} 48-\mathrm{C} 51- \\
\mathrm{F} 23\end{array}$ & $98.6(5)$ \\
\hline $\begin{array}{l}\mathrm{C} 25-\mathrm{C} 24-\mathrm{C} 27- \\
\mathrm{F} 5 \mathrm{~A}\end{array}$ & $-11.4(7)$ & $\begin{array}{l}\mathrm{C} 47-\mathrm{C} 48-\mathrm{C} 51- \\
\mathrm{F} 24 \mathrm{~A}\end{array}$ & $119.7(4)$ \\
\hline $\begin{array}{l}\mathrm{C} 23-\mathrm{C} 24-\mathrm{C} 27- \\
\mathrm{F} 5 \mathrm{~A}\end{array}$ & $169.3(5)$ & $\begin{array}{l}\text { C49-C48-C51- } \\
\text { F24A }\end{array}$ & $-59.5(5)$ \\
\hline $\mathrm{C} 25-\mathrm{C} 24-\mathrm{C} 27-\mathrm{F} 5$ & $7.5(7)$ & $\mathrm{C} 37-\mathrm{C} 36-\mathrm{B} 1-\mathrm{C} 44$ & $37.9(4)$ \\
\hline $\mathrm{C} 23-\mathrm{C} 24-\mathrm{C} 27-\mathrm{F} 5$ & $-171.8(5)$ & $\mathrm{C} 41-\mathrm{C} 36-\mathrm{B} 1-\mathrm{C} 44$ & $-151.6(3)$ \\
\hline $\begin{array}{l}\mathrm{C} 25-\mathrm{C} 24-\mathrm{C} 27- \\
\mathrm{F} 4 \mathrm{~A}\end{array}$ & $103.6(4)$ & $\mathrm{C} 37-\mathrm{C} 36-\mathrm{B} 1-\mathrm{C} 28$ & $157.1(3)$ \\
\hline $\begin{array}{l}\mathrm{C} 23-\mathrm{C} 24-\mathrm{C} 27- \\
\mathrm{F} 4 \mathrm{~A}\end{array}$ & $-75.7(5)$ & $\mathrm{C} 41-\mathrm{C} 36-\mathrm{B} 1-\mathrm{C} 28$ & $-32.4(4)$ \\
\hline $\mathrm{C} 25-\mathrm{C} 24-\mathrm{C} 27-\mathrm{F} 6$ & $-111.8(4)$ & $\mathrm{C} 37-\mathrm{C} 36-\mathrm{B} 1-\mathrm{C} 20$ & $-80.7(3)$ \\
\hline $\mathrm{C} 23-\mathrm{C} 24-\mathrm{C} 27-\mathrm{F} 6$ & $68.9(5)$ & $\mathrm{C} 41-\mathrm{C} 36-\mathrm{B} 1-\mathrm{C} 20$ & $89.9(3)$ \\
\hline $\begin{array}{l}\mathrm{C} 33-\mathrm{C} 28-\mathrm{C} 29- \\
\mathrm{C} 30\end{array}$ & $-3.1(4)$ & $\mathrm{C} 49-\mathrm{C} 44-\mathrm{B} 1-\mathrm{C} 36$ & $-151.5(3)$ \\
\hline $\mathrm{B} 1-\mathrm{C} 28-\mathrm{C} 29-\mathrm{C} 30$ & $-177.6(3)$ & $\mathrm{C} 45-\mathrm{C} 44-\mathrm{B} 1-\mathrm{C} 36$ & $36.1(4)$ \\
\hline $\begin{array}{l}\mathrm{C} 28-\mathrm{C} 29-\mathrm{C} 30- \\
\mathrm{C} 31\end{array}$ & $2.7(5)$ & $\mathrm{C} 49-\mathrm{C} 44-\mathrm{B} 1-\mathrm{C} 28$ & $84.4(3)$ \\
\hline $\begin{array}{l}\mathrm{C} 28-\mathrm{C} 29-\mathrm{C} 30- \\
\mathrm{C} 34\end{array}$ & $-176.0(3)$ & $\mathrm{C} 45-\mathrm{C} 44-\mathrm{B} 1-\mathrm{C} 28$ & $-87.9(3)$ \\
\hline $\begin{array}{l}\mathrm{C} 29-\mathrm{C} 30-\mathrm{C} 31- \\
\mathrm{C} 32\end{array}$ & $0.1(5)$ & $\mathrm{C} 49-\mathrm{C} 44-\mathrm{B} 1-\mathrm{C} 20$ & $-37.6(4)$ \\
\hline $\begin{array}{l}\mathrm{C} 34-\mathrm{C} 30-\mathrm{C} 31- \\
\mathrm{C} 32\end{array}$ & $178.8(3)$ & $\mathrm{C} 45-\mathrm{C} 44-\mathrm{B} 1-\mathrm{C} 20$ & $150.1(3)$ \\
\hline $\begin{array}{l}\mathrm{C} 30-\mathrm{C} 31-\mathrm{C} 32- \\
\mathrm{C} 33\end{array}$ & $-2.2(5)$ & $\mathrm{C} 33-\mathrm{C} 28-\mathrm{B} 1-\mathrm{C} 36$ & $157.4(3)$ \\
\hline $\begin{array}{l}\mathrm{C} 30-\mathrm{C} 31-\mathrm{C} 32- \\
\mathrm{C} 35\end{array}$ & $179.6(3)$ & $\mathrm{C} 29-\mathrm{C} 28-\mathrm{B} 1-\mathrm{C} 36$ & $-28.4(4)$ \\
\hline $\begin{array}{l}\mathrm{C} 31-\mathrm{C} 32-\mathrm{C} 33- \\
\mathrm{C} 28\end{array}$ & $1.7(5)$ & $\mathrm{C} 33-\mathrm{C} 28-\mathrm{B} 1-\mathrm{C} 44$ & $-78.5(3)$ \\
\hline $\begin{array}{l}\mathrm{C} 35-\mathrm{C} 32-\mathrm{C} 33- \\
\mathrm{C} 28\end{array}$ & $179.9(3)$ & $\mathrm{C} 29-\mathrm{C} 28-\mathrm{B} 1-\mathrm{C} 44$ & $95.7(3)$ \\
\hline $\begin{array}{l}\mathrm{C} 29-\mathrm{C} 28-\mathrm{C} 33- \\
\mathrm{C} 32\end{array}$ & $1.0(4)$ & $\mathrm{C} 33-\mathrm{C} 28-\mathrm{B} 1-\mathrm{C} 20$ & $41.4(4)$ \\
\hline $\mathrm{B} 1-\mathrm{C} 28-\mathrm{C} 33-\mathrm{C} 32$ & $175.5(3)$ & $\mathrm{C} 29-\mathrm{C} 28-\mathrm{B} 1-\mathrm{C} 20$ & $-144.4(3)$ \\
\hline $\mathrm{C} 31-\mathrm{C} 30-\mathrm{C} 34-\mathrm{F} 9$ & $-73.0(5)$ & $\mathrm{C} 21-\mathrm{C} 20-\mathrm{B} 1-\mathrm{C} 36$ & $-91.3(3)$ \\
\hline
\end{tabular}




\begin{tabular}{|l|l|l|l|}
\hline $\mathrm{C} 29-\mathrm{C} 30-\mathrm{C} 34-\mathrm{F} 9$ & $105.7(4)$ & $\mathrm{C} 25-\mathrm{C} 20-\mathrm{B} 1-\mathrm{C} 36$ & $80.8(3)$ \\
\hline $\begin{array}{l}\mathrm{C} 31-\mathrm{C} 30-\mathrm{C} 34- \\
\text { F7A }\end{array}$ & $67.8(5)$ & $\mathrm{C} 21-\mathrm{C} 20-\mathrm{B} 1-\mathrm{C} 44$ & $147.8(3)$ \\
\hline $\begin{array}{l}\mathrm{C} 29-\mathrm{C} 30-\mathrm{C} 34- \\
\text { F7A }\end{array}$ & $-113.5(4)$ & $\mathrm{C} 25-\mathrm{C} 20-\mathrm{B} 1-\mathrm{C} 44$ & $-40.0(4)$ \\
\hline $\begin{array}{l}\mathrm{C} 31-\mathrm{C} 30-\mathrm{C} 34- \\
\text { F8A }\end{array}$ & $-168.3(4)$ & $\mathrm{C} 21-\mathrm{C} 20-\mathrm{B} 1-\mathrm{C} 28$ & $31.0(4)$ \\
\hline $\begin{array}{l}\mathrm{C} 29-\mathrm{C} 30-\mathrm{C} 34- \\
\mathrm{F} 8 \mathrm{~A}-\end{array}$ & $10.4(5)$ & $\mathrm{C} 25-\mathrm{C} 20-\mathrm{B} 1-\mathrm{C} 28$ & $-156.9(3)$ \\
\hline $\mathrm{C} 31-\mathrm{C} 30-\mathrm{C} 34-\mathrm{F} 8$ & $162.7(4)$ & $\mathrm{C} 4-\mathrm{C} 3-\mathrm{N} 1-\mathrm{C} 1$ & $173.7(3)$ \\
\hline $\mathrm{C} 29-\mathrm{C} 30-\mathrm{C} 34-\mathrm{F} 8$ & $-18.6(5)$ & $\mathrm{C} 2-\mathrm{C} 3-\mathrm{N} 1-\mathrm{C} 1$ & $-3.9(5)$ \\
\hline $\mathrm{C} 31-\mathrm{C} 30-\mathrm{C} 34-\mathrm{F} 7$ & $46.8(5)$ & $\mathrm{C} 4-\mathrm{C} 3-\mathrm{N} 1-\mathrm{Rh} 1$ & $-5.1(4)$ \\
\hline $\mathrm{C} 29-\mathrm{C} 30-\mathrm{C} 34-\mathrm{F} 7$ & $-134.5(4)$ & $\mathrm{C} 2-\mathrm{C} 3-\mathrm{N} 1-\mathrm{Rh} 1$ & $177.3(3)$ \\
\hline $\begin{array}{l}\mathrm{C} 31-\mathrm{C} 30-\mathrm{C} 34- \\
\text { F9A }\end{array}$ & $-49.8(5)$ & & \\
\hline
\end{tabular}

Document origin: publCIF [Westrip, S. P. (2010). J. Apply. Cryst., 43, 920-925]. 


\section{Computational Analyses}

\section{1}

$\mathrm{E}(\mathrm{RB} 3 \mathrm{LYP}$, triple zeta) $=\mathbf{- 9 7 9 . 2 2 2 2 5 4 4}$

Thermal correction to Free Energy $=0.338541$

\# of imaginary frequency $=0$

\begin{tabular}{|c|c|c|c|}
\hline $\mathrm{Rh}$ & -1.708589000 & -0.477969000 & -0.814293000 \\
\hline $\mathrm{H}$ & -2.415784000 & -2.675946000 & 1.992166000 \\
\hline $\mathrm{H}$ & -1.962932000 & -3.826680000 & -0.085823000 \\
\hline C & -3.071004000 & -1.803220000 & 1.938195000 \\
\hline $\mathrm{H}$ & -4.030272000 & -2.090035000 & 2.389926000 \\
\hline $\mathrm{H}$ & -2.631712000 & -1.018427000 & 2.559044000 \\
\hline $\mathrm{H}$ & -3.675579000 & -4.265114000 & -0.169935000 \\
\hline $\mathrm{C}$ & -2.891380000 & -3.657529000 & -0.638846000 \\
\hline $\mathrm{H}$ & -2.740093000 & -4.034537000 & -1.652849000 \\
\hline $\mathrm{C}$ & -3.254137000 & -1.346407000 & 0.522379000 \\
\hline C & -3.266228000 & -2.207642000 & -0.643606000 \\
\hline $\mathrm{H}$ & -3.383692000 & 1.104455000 & 1.887385000 \\
\hline $\mathrm{C}$ & -3.775615000 & -0.056730000 & 0.120402000 \\
\hline C & -4.081383000 & 1.080139000 & 1.047562000 \\
\hline $\mathrm{C}$ & -3.667531000 & -1.427540000 & -1.752039000 \\
\hline $\mathrm{H}$ & -5.095321000 & 0.985054000 & 1.458425000 \\
\hline $\mathrm{H}$ & -3.278211000 & -2.817230000 & -3.350181000 \\
\hline C & -3.990618000 & -0.091024000 & -1.275991000 \\
\hline C & -3.788672000 & -1.866917000 & -3.179582000 \\
\hline $\mathrm{H}$ & -4.019482000 & 2.043947000 & 0.536424000 \\
\hline $\mathrm{H}$ & -3.348242000 & -1.131153000 & -3.861020000 \\
\hline $\mathrm{H}$ & -4.838709000 & -1.993546000 & -3.473255000 \\
\hline C & -4.568760000 & 1.004035000 & -2.120043000 \\
\hline $\mathrm{H}$ & -4.337902000 & 1.992173000 & -1.712322000 \\
\hline $\mathrm{H}$ & -5.661994000 & 0.923714000 & -2.186098000 \\
\hline $\mathrm{H}$ & -4.188810000 & 0.964597000 & -3.145640000 \\
\hline $\mathrm{H}$ & -2.023303000 & 1.549326000 & -3.113658000 \\
\hline $\mathrm{H}$ & -0.403030000 & -2.531157000 & 1.295764000 \\
\hline C & -1.034084000 & 1.151461000 & -3.305722000 \\
\hline $\mathrm{N}$ & -0.594606000 & 0.235127000 & -2.428107000 \\
\hline C & 0.009298000 & -1.482560000 & -0.541641000 \\
\hline $\mathrm{C}$ & 0.309715000 & -2.380422000 & 0.491612000 \\
\hline $\mathrm{C}$ & -0.275392000 & 1.573864000 & -4.388164000 \\
\hline $\mathrm{H}$ & -0.674111000 & 2.312615000 & -5.074008000 \\
\hline C & 0.648439000 & -0.307208000 & -2.571949000 \\
\hline C & 0.995912000 & -1.278959000 & -1.541191000 \\
\hline C & 1.518249000 & -3.077633000 & 0.515806000 \\
\hline $\mathrm{H}$ & 1.722525000 & -3.773964000 & 1.325471000 \\
\hline C & 1.001693000 & 1.030041000 & -4.554975000 \\
\hline C & 1.463210000 & 0.093070000 & -3.641530000 \\
\hline C & 2.213495000 & -1.978618000 & -1.511350000 \\
\hline $\mathrm{C}$ & 2.473656000 & -2.882594000 & -0.488496000 \\
\hline $\mathrm{H}$ & 1.628892000 & 1.338621000 & -5.385530000 \\
\hline $\mathrm{H}$ & 2.452516000 & -0.335587000 & -3.747931000 \\
\hline $\mathrm{H}$ & 2.958930000 & -1.822021000 & -2.286423000 \\
\hline $\mathrm{H}$ & 3.412961000 & -3.426353000 & -0.466214000 \\
\hline 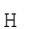 & & 0.551476000 & - \\
\hline
\end{tabular}

\section{$1-17$ e}

$\mathrm{E}(\mathrm{UB} 3 \mathrm{LYP}$, triple zeta) $=-978.6158268$

Thermal correction to Free Energy $=0.326283$

\# of imaginary frequency $=0$

$\begin{array}{lllr}\text { Rh } & -1.797090000 & -0.799247000 & -1.115296000 \\ \mathrm{H} & -2.256210000 & -2.603851000 & 1.972699000 \\ \mathrm{H} & -2.312757000 & -4.008283000 & 0.165907000 \\ \mathrm{C} & -2.847519000 & -1.687274000 & 1.919307000 \\ \mathrm{H} & -3.742413000 & -1.839968000 & 2.537099000 \\ \mathrm{H} & -2.258947000 & -0.887929000 & 2.377603000 \\ \mathrm{H} & -4.029074000 & -4.292586000 & -0.151305000 \\ \mathrm{C} & -3.135288000 & -3.765437000 & -0.510346000 \\ \mathrm{H} & -2.879614000 & -4.171710000 & -1.491991000 \\ \mathrm{C} & -3.218408000 & -1.354713000 & 0.506695000 \\ \mathrm{C} & -3.370490000 & -2.288403000 & -0.578303000 \\ \mathrm{H} & -2.945722000 & 1.201713000 & 1.619558000 \\ \mathrm{C} & -3.681282000 & -0.068984000 & 0.049545000 \\ \mathrm{C} & -3.749359000 & 1.174545000 & 0.878925000 \\ \mathrm{C} & -3.902298000 & -1.570568000 & -1.704658000 \\ \mathrm{H} & -4.701596000 & 1.238295000 & 1.423707000 \\ \mathrm{H} & -3.626091000 & -3.030237000 & -3.258253000 \\ \mathrm{C} & -4.069011000 & -0.200052000 & -1.316360000\end{array}$




$\begin{array}{lrrr}\mathrm{C} & -4.243024000 & -2.155193000 & -3.039486000 \\ \mathrm{H} & -3.664638000 & 2.073727000 & 0.262698000 \\ \mathrm{H} & -4.086128000 & -1.431870000 & -3.844821000 \\ \mathrm{H} & -5.294424000 & -2.470736000 & -3.083462000 \\ \mathrm{C} & -4.629626000 & 0.890728000 & -2.178263000 \\ \mathrm{H} & -4.201458000 & 1.865014000 & -1.923231000 \\ \mathrm{H} & -5.718235000 & 0.975782000 & -2.065444000 \\ \mathrm{H} & -4.432963000 & 0.704667000 & -3.238493000 \\ \mathrm{H} & -2.154971000 & 1.314782000 & -3.355364000 \\ \mathrm{H} & -0.434331000 & -2.952101000 & 0.900033000 \\ \mathrm{C} & -1.099135000 & 1.083994000 & -3.437805000 \\ \mathrm{~N} & -0.633277000 & 0.174931000 & -2.564991000 \\ \mathrm{C} & 0.004867000 & -1.629312000 & -0.745793000 \\ \mathrm{C} & 0.335163000 & -2.583501000 & 0.228678000 \\ \mathrm{C} & -0.292944000 & 1.696377000 & -4.387149000 \\ \mathrm{H} & -0.715153000 & 2.423956000 & -5.070918000 \\ \mathrm{C} & 0.686863000 & -0.178213000 & -2.589906000 \\ \mathrm{C} & 1.060015000 & -1.176641000 & -1.587432000 \\ \mathrm{C} & 1.634647000 & -3.072714000 & 0.367751000 \\ \mathrm{H} & 1.856478000 & -3.811827000 & 1.133813000 \\ \mathrm{C} & 1.060120000 & 1.348070000 & -4.428306000 \\ \mathrm{C} & 1.547010000 & 0.410207000 & -3.526537000 \\ \mathrm{C} & 2.367093000 & -1.668191000 & -1.448214000 \\ \mathrm{C} & 2.656099000 & -2.616824000 & -0.472772000 \\ \mathrm{H} & 1.725730000 & 1.803432000 & -5.155034000 \\ \mathrm{H} & 2.592930000 & 0.128327000 & -3.543408000 \\ \mathrm{H} & 3.164396000 & -1.315581000 & -2.097063000 \\ \mathrm{H} & 3.667210000 & -2.997614000 & -0.365414000 \\ & & & \end{array}$

2

$\mathrm{E}(\mathrm{RB} 3 \mathrm{LYP}$, triple zeta) $=\quad-904.1873851$

Thermal correction to Free Energy $=0.345721$

$\#$ of imaginary frequency

\begin{tabular}{|c|c|c|c|}
\hline $\mathrm{Rh}$ & -1.718665000 & -0.508001000 & -0.847292000 \\
\hline $\mathrm{H}$ & -2.420004000 & -2.653302000 & 1.998560000 \\
\hline $\mathrm{H}$ & -2.024781000 & -3.848491000 & -0.046102000 \\
\hline $\mathrm{C}$ & -3.067845000 & -1.775311000 & 1.940120000 \\
\hline $\mathrm{H}$ & -4.023122000 & -2.046138000 & 2.409917000 \\
\hline $\mathrm{H}$ & -2.612194000 & -0.985738000 & 2.542889000 \\
\hline $\mathrm{H}$ & -3.741526000 & -4.262608000 & -0.159481000 \\
\hline C & -2.940449000 & -3.667450000 & -0.616042000 \\
\hline $\mathrm{H}$ & -2.776591000 & -4.050513000 & -1.625941000 \\
\hline $\mathrm{C}$ & -3.266383000 & -1.338169000 & 0.520167000 \\
\hline $\mathrm{C}$ & -3.294204000 & -2.212152000 & -0.633282000 \\
\hline $\mathrm{H}$ & -3.349337000 & 1.129588000 & 1.852599000 \\
\hline $\mathrm{C}$ & -3.774438000 & -0.048573000 & 0.104454000 \\
\hline C & -4.055350000 & 1.104503000 & 1.019757000 \\
\hline C & -3.702278000 & -1.442902000 & -1.749458000 \\
\hline $\mathrm{H}$ & -5.066467000 & 1.029194000 & 1.441553000 \\
\hline $\mathrm{H}$ & -3.294781000 & -2.828339000 & -3.347865000 \\
\hline $\mathrm{C}$ & -4.008296000 & -0.098391000 & -1.289426000 \\
\hline $\mathrm{C}$ & -3.871325000 & -1.919712000 & -3.160070000 \\
\hline $\mathrm{H}$ & -3.985020000 & 2.060860000 & 0.495960000 \\
\hline $\mathrm{H}$ & -3.540258000 & -1.164974000 & -3.880552000 \\
\hline $\mathrm{H}$ & -4.923227000 & -2.139158000 & -3.385827000 \\
\hline C & -4.593226000 & 0.992076000 & -2.134904000 \\
\hline $\mathrm{H}$ & -4.370559000 & 1.980751000 & -1.724832000 \\
\hline $\mathrm{H}$ & -5.685695000 & 0.902151000 & -2.202371000 \\
\hline $\mathrm{H}$ & -4.207374000 & 0.962549000 & -3.157749000 \\
\hline $\mathrm{H}$ & -0.416297000 & -2.587463000 & 1.262485000 \\
\hline C & -1.162475000 & 1.173337000 & -3.335379000 \\
\hline $\mathrm{N}$ & -0.578818000 & 0.183906000 & -2.450925000 \\
\hline C & -0.006039000 & -1.506490000 & -0.558083000 \\
\hline C & 0.301521000 & -2.409392000 & 0.468306000 \\
\hline C & 0.624079000 & -0.299436000 & -2.568553000 \\
\hline C & 0.989055000 & -1.271529000 & -1.544333000 \\
\hline C & 1.526751000 & -3.077476000 & 0.498619000 \\
\hline $\mathrm{H}$ & 1.736297000 & -3.779970000 & 1.301775000 \\
\hline $\mathrm{C}$ & 1.616786000 & 0.076143000 & -3.636586000 \\
\hline $\mathrm{C}$ & 2.226247000 & -1.936598000 & -1.504343000 \\
\hline C & 2.493362000 & -2.846168000 & -0.487480000 \\
\hline $\mathrm{H}$ & 2.978954000 & -1.746295000 & -2.264314000 \\
\hline $\mathrm{H}$ & 3.445745000 & -3.366221000 & -0.454961000 \\
\hline $\mathrm{H}$ & -0.459545000 & 1.601779000 & -4.054461000 \\
\hline $\mathrm{H}$ & -1.579240000 & 1.979279000 & -2.726014000 \\
\hline $\mathrm{H}$ & -1.990758000 & 0.717162000 & -3.888293000 \\
\hline $\mathrm{H}$ & 1.231571000 & 0.789852000 & -4.362177000 \\
\hline $\mathrm{H}$ & 1.929671000 & -0.821740000 & -4.178836000 \\
\hline 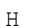 & 2.514226000 & 0.503019000 & -3.176922000 \\
\hline
\end{tabular}




\begin{tabular}{|c|c|c|c|}
\hline $\mathrm{H}$ & -0.953058000 & 0.539897000 & 0.021928000 \\
\hline \multicolumn{4}{|c|}{$\begin{array}{l}\text { E (UB3LYP, triple zeta) }=-903.5797758 \\
\text { Thermal correction to Free Energy }=0.333518 \\
\# \text { of imaginary frequency }=0\end{array}$} \\
\hline & -1.790825000 & -0.788484000 & -1.069343000 \\
\hline & -2.385606000 & -2.692816000 & 1.940116000 \\
\hline & -2.385087000 & -4.018105000 & 0.067667000 \\
\hline & -2.949253000 & -1.758024000 & 1.903518000 \\
\hline & -3.864595000 & -1.906492000 & 2.491765000 \\
\hline & -2.351547000 & -0.992484000 & 2.405820000 \\
\hline & -4.105200000 & -4.266019000 & -0.263296000 \\
\hline & -3.201922000 & -3.742185000 & -0.603235000 \\
\hline & -2.949541000 & -4.122550000 & -1.596094000 \\
\hline & -3.270716000 & -1.367260000 & 0.493467000 \\
\hline & -3.411886000 & -2.259857000 & -0.626970000 \\
\hline & -2.977813000 & 1.140200000 & 1.703355000 \\
\hline & -3.694746000 & -0.057735000 & 0.068849000 \\
\hline & -3.760985000 & 1.156618000 & 0.940765000 \\
\hline & -3.893710000 & -1.491605000 & -1.741975000 \\
\hline & -4.726397000 & 1.220313000 & 1.461892000 \\
\hline & -3.595465000 & -2.892589000 & -3.345853000 \\
\hline & -4.046172000 & -0.131594000 & -1.310983000 \\
\hline & -4.211146000 & -2.022349000 & -3.104873000 \\
\hline & -3.641861000 & 2.075531000 & 0.360414000 \\
\hline & -4.035791000 & -1.268575000 & -3.878140000 \\
\hline $\mathrm{H}$ & -5.263447000 & -2.328413000 & -3.182269000 \\
\hline c & -4.569680000 & 0.997249000 & -2.146738000 \\
\hline $\mathrm{H}$ & -4.159976000 & 1.958868000 & -1.824040000 \\
\hline $\mathrm{H}$ & -5.663241000 & 1.071331000 & -2.083708000 \\
\hline $\mathrm{H}$ & -4.315664000 & 0.867122000 & -3.202385000 \\
\hline $\mathrm{H}$ & -0.489763000 & -2.982896000 & 0.974788000 \\
\hline C & -1.163167000 & 1.062759000 & -3.439022000 \\
\hline $\mathrm{N}$ & -0.569006000 & 0.121408000 & -2.508912000 \\
\hline C & -0.019058000 & -1.663302000 & -0.664949000 \\
\hline C & 0.289593000 & -2.620620000 & 0.310830000 \\
\hline c & 0.688294000 & -0.226039000 & -2.507167000 \\
\hline c & 1.050598000 & -1.221374000 & -1.495851000 \\
\hline c & 1.584693000 & -3.123527000 & 0.460519000 \\
\hline H & 1.790425000 & -3.867291000 & 1.226792000 \\
\hline c & 1.741392000 & 0.301434000 & -3.445194000 \\
\hline C & 2.352382000 & -1.723472000 & -1.344519000 \\
\hline C & 2.620598000 & -2.677481000 & -0.366339000 \\
\hline $\mathrm{H}$ & 3.158412000 & -1.376550000 & -1.985168000 \\
\hline $\mathrm{H}$ & 3.626058000 & -3.069680000 & -0.247462000 \\
\hline $\mathrm{H}$ & -0.451919000 & 1.542293000 & -4.116989000 \\
\hline $\mathrm{H}$ & -1.683567000 & 1.839194000 & -2.870832000 \\
\hline $\mathrm{H}$ & -1.916173000 & 0.539301000 & -4.038083000 \\
\hline $\mathrm{H}$ & 1.374663000 & 1.056137000 & -4.138024000 \\
\hline $\mathrm{H}$ & 2.161957000 & -0.523361000 & -4.030095000 \\
\hline $\mathrm{H}$ & 2.564178000 & 0.737340000 & -2.869617000 \\
\hline
\end{tabular}

3

$\mathrm{E}(\mathrm{RB} 3 \mathrm{LYP}$, triple zeta $)=-981.6331538$

\# of imaginary frequency $=0.383781$

$\begin{array}{llrr}\text { Rh } & -1.713605000 & -0.513772000 & -0.828609000 \\ \text { H } & -2.428924000 & -2.649160000 & 2.012560000 \\ \text { H } & -2.010121000 & -3.854227000 & -0.021122000 \\ \text { C } & -3.094169000 & -1.784934000 & 1.943429000 \\ \text { H } & -4.051108000 & -2.075423000 & 2.397920000 \\ \text { H } & -2.664616000 & -0.986237000 & 2.553324000 \\ \text { H } & -3.721950000 & -4.279283000 & -0.164477000 \\ \text { C } & -2.916674000 & -3.679428000 & -0.607295000 \\ \text { H } & -2.732578000 & -4.062049000 & -1.613880000 \\ \text { C } & -3.277245000 & -1.351314000 & 0.520347000 \\ \text { C } & -3.279478000 & -2.226500000 & -0.632035000 \\ \text { H } & -3.392282000 & 1.120658000 & 1.844293000 \\ \text { C } & -3.786208000 & -0.065250000 & 0.094128000 \\ \text { C } & -4.087706000 & 1.087913000 & 1.002819000 \\ \text { C } & -3.672824000 & -1.461528000 & -1.756763000 \\ \text { H } & -5.103362000 & 1.006084000 & 1.412287000 \\ \text { H } & -3.216235000 & -2.838269000 & -3.349320000 \\ \text { C } & -3.994852000 & -0.118407000 & -1.303779000 \\ \text { C } & -3.812788000 & -1.940868000 & -3.169763000 \\ \text { H } & -4.018041000 & 2.043512000 & 0.477534000 \\ \text { H } & -3.485089000 & -1.179056000 & -3.884155000 \\ \text { H } & -4.856870000 & -2.180495000 & -3.411095000\end{array}$




$\begin{array}{lrrr}\mathrm{C} & -4.561922000 & 0.968970000 & -2.165206000 \\ \mathrm{H} & -4.370060000 & 1.957814000 & -1.740163000 \\ \mathrm{H} & -5.649396000 & 0.864409000 & -2.277213000 \\ \mathrm{H} & -4.133614000 & 0.951495000 & -3.171451000 \\ \mathrm{H} & -0.404231000 & -2.591043000 & 1.282855000 \\ \mathrm{C} & -1.111606000 & 1.204414000 & -3.319873000 \\ \mathrm{~N} & -0.572471000 & 0.192134000 & -2.414979000 \\ \mathrm{C} & 0.003237000 & -1.507845000 & -0.537006000 \\ \mathrm{C} & 0.314059000 & -2.409031000 & 0.489695000 \\ \mathrm{C} & 0.626050000 & -0.298655000 & -2.541869000 \\ \mathrm{C} & 0.998623000 & -1.270631000 & -1.523941000 \\ \mathrm{C} & 1.543466000 & -3.069713000 & 0.522041000 \\ \mathrm{H} & 1.756070000 & -3.770451000 & 1.325957000 \\ \mathrm{C} & 1.585123000 & 0.118261000 & -3.632519000 \\ \mathrm{C} & 2.239458000 & -1.928123000 & -1.482177000 \\ \mathrm{C} & 2.510603000 & -2.833594000 & -0.462566000 \\ \mathrm{H} & 2.992620000 & -1.732842000 & -2.240584000 \\ \mathrm{H} & 3.466222000 & -3.347498000 & -0.427025000 \\ \mathrm{H} & -0.958596000 & 0.531857000 & 0.054042000 \\ \mathrm{C} & 0.908213000 & 0.909076000 & -4.755011000 \\ \mathrm{C} & -0.050543000 & 1.930223000 & -4.144800000 \\ \mathrm{H} & 2.076931000 & -0.776329000 & -4.027929000 \\ \mathrm{H} & 2.380292000 & 0.719222000 & -3.169672000 \\ \mathrm{H} & -0.539315000 & 2.535285000 & -4.915735000 \\ \mathrm{H} & 0.507776000 & 2.618488000 & -3.497462000 \\ \mathrm{H} & -1.686350000 & 1.905547000 & -2.709059000 \\ \mathrm{H} & -1.831874000 & 0.704613000 & -3.983013000 \\ \mathrm{H} & 0.345047000 & 0.223607000 & -5.401365000 \\ \mathrm{H} & 1.662457000 & 1.392889000 & -5.383375000 \\ & & & \end{array}$

\section{3-17e}

$\mathrm{E}($ UB 3LYP, triple zeta) $=-981.0255089$

Thermal correction to Free Energy $=0.371039$

\# of imaginary frequency =

$\begin{array}{lrrr}\text { Rh } & -1.859113000 & -1.067886000 & -1.349760000 \\ \mathrm{H} & -2.019427000 & -2.246633000 & 1.917170000 \\ \mathrm{H} & -2.684833000 & -3.996503000 & 0.596263000 \\ \mathrm{C} & -2.709976000 & -1.404722000 & 1.828863000 \\ \mathrm{H} & -3.539234000 & -1.577832000 & 2.527619000 \\ \mathrm{H} & -2.175920000 & -0.510594000 & 2.159778000 \\ \mathrm{H} & -4.374041000 & -4.186645000 & 0.126441000 \\ \mathrm{C} & -3.403369000 & -3.790259000 & -0.199949000 \\ \mathrm{H} & -3.091197000 & -4.357864000 & -1.080833000 \\ \mathrm{C} & -3.206313000 & -1.252061000 & 0.424500000 \\ \mathrm{C} & -3.489774000 & -2.323789000 & -0.493621000 \\ \mathrm{H} & -2.677726000 & 1.401736000 & 1.125593000 \\ \mathrm{C} & -3.584106000 & -0.018708000 & -0.210169000 \\ \mathrm{C} & -3.488239000 & 1.347089000 & 0.394530000 \\ \mathrm{C} & -4.064933000 & -1.745523000 & -1.680380000 \\ \mathrm{H} & -4.418332000 & 1.625890000 & 0.908492000 \\ \mathrm{H} & -3.972776000 & -3.421292000 & -3.023550000 \\ \mathrm{C} & -4.093067000 & -0.328971000 & -1.514682000 \\ \mathrm{C} & -4.558020000 & -2.511165000 & -2.867665000 \\ \mathrm{H} & -3.298321000 & 2.109454000 & -0.366841000 \\ \mathrm{H} & -4.498466000 & -1.915450000 & -3.782532000 \\ \mathrm{H} & -5.607152000 & -2.812281000 & -2.739454000 \\ \mathrm{C} & -4.643088000 & 0.658043000 & -2.499823000 \\ \mathrm{H} & -4.179998000 & 1.642031000 & -2.384232000 \\ \mathrm{H} & -5.725003000 & 0.794176000 & -2.371947000 \\ \mathrm{H} & -4.474752000 & 0.332706000 & -3.530534000 \\ \mathrm{H} & -0.602911000 & -3.461076000 & 0.500885000 \\ \mathrm{C} & -1.075846000 & 1.257960000 & -3.255059000 \\ \mathrm{~N} & -0.569287000 & 0.133144000 & -2.471060000 \\ \mathrm{C} & -0.076514000 & -1.904200000 & -0.895908000 \\ \mathrm{C} & 0.206181000 & -2.971344000 & -0.033111000 \\ \mathrm{C} & 0.693431000 & -0.192534000 & -2.448242000 \\ \mathrm{C} & 1.030002000 & -1.314621000 & -1.574093000 \\ \mathrm{C} & 1.511662000 & -3.431891000 & 0.159965000 \\ \mathrm{H} & 1.697203000 & -4.260192000 & 0.839843000 \\ \mathrm{C} & 1.755544000 & 0.534690000 & -3.239731000 \\ \mathrm{C} & 2.340317000 & -1.779516000 & -1.386268000 \\ \mathrm{C} & 2.583143000 & -2.838363000 & -0.514903000 \\ \mathrm{H} & 3.172690000 & -1.318305000 & -1.910972000 \\ \mathrm{H} & 3.595784000 & -3.199484000 & -0.363000000 \\ \mathrm{C} & 1.176725000 & 1.469564000 & -4.304625000 \\ \mathrm{C} & 0.000569000 & 2.242015000 & -3.709773000 \\ \mathrm{H} & 2.424120000 & -0.205358000 & -3.691579000 \\ \mathrm{H} & 2.373705000 & 1.102963000 & -2.530361000 \\ \mathrm{H} & -0.428074000 & 2.944220000 & -4.432623000\end{array}$




$\begin{array}{rrrr}\mathrm{H} & 0.345819000 & 2.831812000 & -2.850672000 \\ \mathrm{H} & -1.830594000 & 1.759741000 & -2.642661000 \\ \mathrm{H} & -1.609099000 & 0.844760000 & -4.122692000 \\ \mathrm{H} & 0.827324000 & 0.880320000 & -5.162074000 \\ \mathrm{H} & 1.953634000 & 2.144818000 & -4.676563000\end{array}$

H

$\mathrm{E}(\mathrm{B} 3 \mathrm{LYP}$, triple zeta $)=-0.50215593$

Thermal correction to Free Energy $=-0.010654$

Double $\mathbf{H}$ abstract intermediate of $\mathbf{2}$ toward the formation of $\mathbf{5}$

$\mathrm{E}(\mathrm{B} 3 \mathrm{LYP}, \mathrm{triple}$ zeta $)=\quad-902.9613707$

Thermal correction to Free Energy $=0.32726$

$\begin{array}{lrrr}\mathrm{Rh} & -1.817722000 & -0.923233000 & -1.232389000 \\ \mathrm{H} & -2.429099000 & -3.266621000 & 1.540978000 \\ \mathrm{H} & -2.799393000 & -4.282079000 & -0.482161000 \\ \mathrm{C} & -2.957715000 & -2.320405000 & 1.678797000 \\ \mathrm{H} & -3.894886000 & -2.542594000 & 2.206228000 \\ \mathrm{H} & -2.354598000 & -1.691292000 & 2.337692000 \\ \mathrm{H} & -4.513292000 & -4.209728000 & -0.919943000 \\ \mathrm{C} & -3.524876000 & -3.773516000 & -1.120909000 \\ \mathrm{H} & -3.269282000 & -4.000371000 & -2.159223000 \\ \mathrm{C} & -3.250217000 & -1.645477000 & 0.372385000 \\ \mathrm{C} & -3.534684000 & -2.295751000 & -0.870315000 \\ \mathrm{H} & -2.524847000 & 0.557886000 & 1.953785000 \\ \mathrm{C} & -3.416333000 & -0.240122000 & 0.163348000 \\ \mathrm{C} & -3.259780000 & 0.835874000 & 1.195117000 \\ \mathrm{C} & -4.047621000 & -1.293194000 & -1.805424000 \\ \mathrm{H} & -4.213496000 & 1.028408000 & 1.705955000 \\ \mathrm{H} & -3.928636000 & -2.324108000 & -3.695087000 \\ \mathrm{C} & -3.974327000 & -0.038883000 & -1.175230000 \\ \mathrm{C} & -4.566695000 & -1.601142000 & -3.178461000 \\ \mathrm{H} & -2.931567000 & 1.776074000 & 0.744296000 \\ \mathrm{H} & -4.626499000 & -0.705494000 & -3.801559000 \\ \mathrm{H} & -5.574757000 & -2.033020000 & -3.127202000 \\ \mathrm{C} & -4.397867000 & 1.290206000 & -1.726280000 \\ \mathrm{H} & -3.674887000 & 2.073940000 & -1.481964000 \\ \mathrm{H} & -5.365440000 & 1.596189000 & -1.307294000 \\ \mathrm{H} & -4.505975000 & 1.264707000 & -2.813337000 \\ \mathrm{H} & -0.418069000 & -2.293320000 & 1.407788000 \\ \mathrm{C} & -1.252478000 & 0.357008000 & -3.905659000 \\ \mathrm{~N} & -0.705872000 & -0.260869000 & -2.711204000 \\ \mathrm{C} & 0.033642000 & -1.387788000 & -0.504199000 \\ \mathrm{C} & 0.361159000 & -2.000833000 & 0.713206000 \\ \mathrm{C} & 0.678893000 & -0.383834000 & -2.633235000 \\ \mathrm{C} & 1.095137000 & -1.027212000 & -1.369447000 \\ \mathrm{C} & 1.690061000 & -2.251440000 & 1.066821000 \\ \mathrm{H} & 1.916112000 & -2.728520000 & 2.017823000 \\ \mathrm{C} & 1.536827000 & 0.034922000 & -3.596988000 \\ \mathrm{C} & 2.425929000 & -1.279359000 & -1.013065000 \\ \mathrm{C} & 2.726008000 & -1.890591000 & 0.202387000 \\ \mathrm{H} & 3.235491000 & -0.999801000 & -1.681904000 \\ \mathrm{H} & 3.760375000 & -2.083586000 & 0.473368000 \\ \mathrm{H} & -0.886791000 & 1.386760000 & -4.034809000 \\ \mathrm{H} & -2.339893000 & 0.389560000 & -3.833491000 \\ \mathrm{H} & -0.986070000 & -0.205116000 & -4.813379000 \\ \mathrm{H} & 1.204551000 & 0.503733000 & -4.513756000 \\ \mathrm{H} & 2.605843000 & -0.088242000 & -3.479532000\end{array}$




\section{References}

1. Pangborn, A. B.; Giardello, M. A.; Grubbs, R. H.; Rosen, R. K.; Timmers, F. J. Safe and Convenient Procedure for Solvent Purification. Organometallics 1996, 15, 1518-1520.

2. Lefranc, J.; Tetlow, D. J.; Donnard, M.; Minassi, A.; Gálvez, E.; Clayden, J. GeometrySelective Synthesis of $E$ or ZN-Vinyl Ureas (N-Carbomoyl Enamines). Org. Lett. 2011, 13, 296-299.

3. Hu, Y.; Li, L.; Shaw, A. P.; Norton, J. R.; Sattler, W.; Rong, Y. Synthesis, Electrochemistry, and Reactivity of New Iridium(III) and Rhodium(III) Hydrides. Organometallics 2012, 31, 5058-5064.

4. (a) Du Bois, J.; Hong, J.; Carreira, E. M.; Day, M. W. Nitrogen Transfer from a Nitridomanganese(V) Complex: Amination of Silyl Enol Ethers. J. Am. Chem. Soc. 1996, 118, 915-916. (b) Chang, C. J.; Connick, W. B.; Low, D. W.; Day, M. W.; Gray, H. B. Electronic Structures of Nitridomanganese(V) Complexes. Inorg. Chem. 1998, 37, 31073110.

5. Wang, D.; Loose, F.; Chirik, P. J.; Knowles, R. R. N-H Bond Formation in a Manganese(V) Nitride Yields Ammonia by Light-Driven Proton-Coupled Electron Transfer. J. Am. Chem. Soc. 2019, 141, 4795-4799.

6. Stoll, S.; Schweiger, A. EasySpin, a comprehensive software package for spectral simulatioin and analysis in EPR. J. Magn. Reson. 2006, 178, 42-55.

7. Frisch, M. J.; Trucks, G. W.; Schlegel, H. B.; Scuseria, G. E.; Robb, M. A.; Cheeseman, J. R.; Scalmani, G.; Barone, V.; Mennucci, B.; Petersson, G. A.; Nakatsuji, H.; Caricato, M.; Li, X.; Hratchian, H. P.; Izmaylov, A. F.; Bloino, J.; Zheng, G.; Sonnenberg, Hada, M.; Ehara, M.; Toyota, K.; Fukuda, R.; Hasegawa, J.; Ishida, M.; Nakajima, T.; Honda, Y.; Kitao, O.; Nakai, H.; Vreven, T.; Montgomery, Jr., J. A.; Peralta, J. E.; Ogaliaro, F.; Bearpark, M.; Heyd, J. J.; Brothers, E.; Kudin, K. N.; Staroverov, V. N.; Keith, T.; Kobayashi, R.; Normand, J.; Raghavachari, K.; Rendell, A.; Burant, J. C.; lyengar, S. S.; Tomasi, J.; Cossi, M.; Rega, N.; Millam, J. M.; Klene, M.; Knox, J. E.; Cross, J. B.; Bakken, V.; Adamo, C.; Jaramillo, J.; Gomperts, R.; Stratmann, R. E.; Yazyev, O.; Austin, A. J.; Cammi, R.; Pomelli, C.; Ochterski, J. W.; Martin, R. L.; Morokuma, K.; Zakrzewski, V. G.; Voth, G. A.; Salvador, P.; Dannenberg, J. J.; Dapprich, S.; Daniels, A. D.; Farkas, O.; Foresman, J. B.; Ortiz, J. V.; Cioslowski, J.; Fox, D. J. Gaussian 09, Revision D.01, Gaussian, Inc., Wallingford CT, 2013.

8. Slater, J. C. Quantum Theory of Molecules and Solids, Vol. 4: The Self-Consistent Field for Molecules and Solids, McGraw-Hill, New York, 1974.

9. Vosko, S. H.; Wilk, L.; Nusair, M. Accurate Spin-Dependent Electron Liquid Correlation Energies for Local Spin Density Calculations: A Critical Analysis. Can. J. Phys. 1980, 58, 1200-1211.

10. Becke, A. D. Density-Functional Exchange-Energy Approximation with Correct Asymptotic Behavior. Phys. Rev. A 1988, 38, 3098-3100. 
11. Lee, C; Yang, W.; Parr, R. G. Development of the Colle-Salvetti Correlation-Energy Formula into a Functional of the Electron Density. Phys. Rev. B 1988, 37, 785-789.

12. Becke, A. D. Density-Functional Thermochemistry. III. The Role of Exact Exchange. J. Chem. Phys. 1993, 98, 5648-5652.

13. Hay, P. J.; Wadt, W. R. Ab Initio Effective Core Potentials for Molecular Calculations. Potentials for the Transition Metal Atoms Sc to Hg. J. Chem. Phys. 1985, 82, 270-283.

14. Ehlers, A. W.; Böhme, M.; Dapprich, S.; Gobbi, A.; Höllwarth, A.; Jonas, V.; Köhler, K. F.; Stegmann, R.; Veldkamp, A. A Set of f-Polarization Functions for Pseudo-Potential Basis Sets of the Transition Metals Sc-Cu, Y-Ag and La-Au. J. Chem. Phys. Lett. 1993, 208, 111-114.

15. Hay, P. J.; Wadt, W. R. Ab Initio Effective Core Potentials for Molecular Calculations. Potentials for $\mathrm{K}$ to Au Including the Outermost Core Orbitals. J. Chem. Phys. 1985, 82, 299-310.

16. Roy, L. E.; Hay, P. J.; Martin, R. L. Revised Basis Sets for the LANL Effective Core Potentials. J. Chem. Theory Comput. 2008, 4, 1029-1031.

17. Li, L. Brennessel, W. W.; Jones, W. D. An Efficient Low-Temperature Rout to Polycyclic Isoquinoline Salt Synthesis via C-H Activation with $\left[\mathrm{Cp}^{*} \mathrm{MCl}_{2}\right]_{2}(\mathrm{M}=\mathrm{Rh}, \mathrm{Ir})$. J. Am. Chem. Soc. 2008, 130, 12414-12419.

18. $\mathrm{Hu}, \mathrm{Y}$;; Norton, J. R. Kinetics and Thermodynamics of $\mathrm{H}^{-} / \mathrm{H} \cdot / \mathrm{H}^{+}$Transfer from a Rhodium(III) Hydride. J. Am. Chem. Soc. 2014, 136, 5938-5948. 